\title{
Numerical Simulations of
}

\section{Polymers at the Nanoscale}

by

Srikanth Dhondi

\author{
A thesis submitted to \\ the Victoria University of Wellington \\ in fulfilment of the requirements for the degree of \\ Doctor of Philosophy in Physics \\ Victoria University of Wellington \\ The MacDiarmid Institute for Advanced Materials \\ and Nanotechnology \\ 2011
}





\begin{abstract}
In this thesis we study a variety of nanoscale phenomena in certain polymer systems using a combination of numerical simulation methods and mathematical modelling. The problems considered are: (a) the mixing behaviour of polymeric fluids in micro- and nanofluidic devices, (b) capillary absorption of polymer droplets into narrow capillaries, and (c) modelling the phase separation and self-assembly behaviour in polymer systems with freely deforming boundaries. These problems are significant in nanotechnological applications of polymer-based systems.
\end{abstract}

First, the mixing behaviour of a polymeric melt over two parallely patternedslip surfaces is considered. Using molecular dynamics (MD) simulations, it is shown that mixing is enhanced when the polymer chain size is smaller than the wavelength of the chemical pattern of the surfaces. An off-set in the upper and lower wall patterns improved the mixing in the centre of the channel. Application of a sinusoidally varying body force in addition to the patterned-slip conditions is shown to enhance mixing further, compared to a constant body force case, with some limitations. Simulation findings for the constant body force cases are in qualitative agreement with the continuum theory of Pereira [1]. However, in the case of a sinusoidally varying body force our simulations do not agree with the continuum theory. We explain the reasons for the discrepancy between the two and point out the deficiencies in the continuum theory in predicting the correct behaviour.

Second, the capillary phenomena of polymer droplets in narrow capillaries is studied using MD simulations. It is demonstrated that droplets composed of longer chains require wider tubes for absorption and this result is in agreement with our continuum modelling. The observed capillary dynamics deviate significantly from the standard Lucas-Washburn description thus questioning its validity at the nanoscale. The metastable states during the capillary absorption in some cases cannot be explained using the existing models of capillary dynamics.

Lastly, the phase separation process in polymer blends between both confined and unconfined boundaries is studied using Smoothed Particle Hydrodynamics $(\mathrm{SPH})$. The SPH technique has the advantage of not using a grid to discretize the spatial domain, which makes it appealing when dealing with problems where the spatial domain can change with time. The applicability of the SPH method in describing phase separation in these systems is demonstrated. In particular, its ability to model freely deforming polymer blends is shown. 



\section{Acknowledgements}

I am deeply indebted to my supervisors, Shaun Hendy and Gerald Pereira, for their guidance and constant encouragement, without which this thesis would not have been possible. Shaun always found time when I needed to discuss things with him and his inputs have been invaluable. In particular, I would like to thank him for his support during the tough times I experienced working on this thesis. Throughout this thesis, I have worked closely with Gerald. His constant support, enthusiasm, and insightful comments made this work possible. I appreciate his efforts in ensuring that I had a smooth transition when I first arrived both in Wellington and Melbourne. I would also like to thank both my supervisors for providing me with an opportunity to spend time at CSIRO, Melbourne. Apart from my supervisors, I wish to thank Dmitri for the many discussions on physics, and Peter McGavin for his help with computational issues. I am thankful to the MacDiarmid Institute for funding this thesis, and to CSIRO for their financial help.

Much needed distractions are important in a graduate student's life. During my thesis I made some good friends, without whom this journey would have been pale and boring. In particular, I am extremely grateful to Dmitri, Shrivs and Achïm for all their love and for their support when things looked gloomy. Their support in the days leading up to my thesis completion has been invaluable and something that I will always remember. I was lucky to have a friend like Peter who was always there for me, ready to help. My stay in Melbourne was a pleasurable experience thanks to friends Sergiu, Dhananjay aka DJ and Mandar. Raj and Anu, Bhoom anna and Suvarna, Rampy, and Alok'ji: thank you for your friendship, warmth and hospitality. A special thanks to the badminton club members at Vic and Monash for keeping me in good shape and Achïm for introducing me to tramping. A very special thank you to South Park, Kurosawa, Nirvana, and Ram Gopal Varma's blog.

A great number of people have supported me during my years in India, and without their love and support I would not have been able to reach here. Srikanth and Priya: thank you for being pillars of strength; and for your love and faith in me. Naresh and Rama Krishna, my childhood buddies: thank you for always being there for me. Kiran, Bharath, Mayi, Prasanna, Venki, Swetha, Bhoja Raju, Shyla, Shiva and Aruna: thank you for all your love. I wish to express my special gratitude to Kiran, Srikanth, Bhoja Raju, Rama Krishna, Mayi and his family, Naresh and his family for taking care of my mother needs while I was away. 
I find myself lost for words when I try to express my gratitude towards my mother. I will forever be indebted to her for her selfless love, encouragement, sacrifices and support all these years. Love you maa! 


\section{Contents}

1 Introduction 1

2 Polymer Physics $\quad 6$

2.1 Basic Concepts in Polymer Physics . . . . . . . . . . . . . . . 7

2.2 Ideal Chain Models . . . . . . . . . . . . . . . . . . . 9

2.2.1 Freely Jointed Chain (FJC) Model . . . . . . . . . . . . . . . 9

2.2.2 Freely Rotating Chain (FRC) Model . . . . . . . . . . . . . 12

2.2.3 The Gaussian Chain Model . . . . . . . . . . . . . . . . . . 13

2.2.4 Limitations of Ideal Chain Models . . . . . . . . . . . . . . . 14

2.3 Real Polymers . . . . . . . . . . . . . . . . . . . 15

2.3.1 Excluded Volume Effect . . . . . . . . . . . . . . 15

2.3.2 Probability Distribution for $R \ldots \ldots \ldots$

2.3.3 A Polymer Chain in a Melt is Ideal . . . . . . . . . . . . . . . 19

2.4 Radius of Gyration . . . . . . . . . . . . . . . . . . . . . 19

2.5 Viscoelasticity ........................ 20

3 Molecular Dynamics $\quad 22$

3.1 Connection to Statistical Mechanics . . . . . . . . . . . . . . . . . 24

3.2 Implementation . . . . . . . . . . . . . . . . 25

3.2 .1 Initialization . . . . . . . . . . . 25

3.2 .2 Potentials ........................ 27 
3.2 .3 Coarse Graining . . . . . . . . . . . . . . 30

3.2.4 The Finitely Extensible Nonlinear Elastic (FENE) Potential . 32

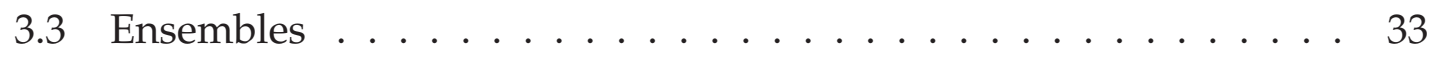

3.3.1 Microcanonical Ensemble (NVE) . . . . . . . . . . . . 33

3.3.2 Canonical Ensemble (NVT) . . . . . . . . . . . . . 34

3.3.3 Application to Nonequilibrium Molecular Dynamics . . . . 38

3.4 Time integration . . . . . . . . . . . . . . 38

3.4.1 Predictor-Corrector Method . . . . . . . . . . . . . 40

3.4 .2 Verlet Algorithm .................. 41

3.5 Tools ................................ 42

3.5.1 Periodic Boundary Conditions . . . . . . . . . . . 42

3.5.2 Limiting the Interaction Range . . . . . . . . . . . . . 43

3.5.3 Neighbour-list Building . . . . . . . . . . . . 43

3.5.4 Parallelization ................... 44

4 Flow over Patterned Surfaces $\quad 47$

4.1 Mixing Methods . . . . . . . . . . . . . . . . . . . 48

4.2 Micro- and Nanoscale Flows . . . . . . . . . . . . . . . . . . . . 49

4.2 .1 Laminar Flow . . . . . . . . . . . . . . . . . . 49

4.2.2 Surface Area to Volume Ratio . . . . . . . . . . . . . . . 50

4.3 Concept of Slip Length . . . . . . . . . . . . . . . . . . . 50

4.4 Molecular Dynamics Simulations . . . . . . . . . . . . . . . 51

4.5 Characterization of The Fluid . . . . . . . . . . . 55

4.5 .1 Couette Flow . . . . . . . . . . . . . 55

4.6 Determining the Slip Lengths . . . . . . . . . . . . . . . . . 60

4.7 MD Simulations for Enhanced Mixing . . . . . . . . . . . . . 62

4.7.1 Patterned Boundaries ............... 62 
4.7 .2 Off-Set Patterning . . . . . . . . . . . . . . 67

4.7.3 Comparison with Continuum Modelling . . . . . . . . . 68

4.8 Time Dependent Body Force . . . . . . . . . . . . . . . . . . . . . 70

4.8.1 Simulations of Homogeneous Channels with Sinusoidal Body Force . . . . . . . . . . . . . . . . 70

4.8.2 Simulations of Patterned Channels with Sinusoidal Body Force ........................ 72

4.8.3 Comparison between Constant Body Force and Sinusoidal Body Force Simulations . . . . . . . . . . . . . . . . 73

4.9 Summary . . . . . . . . . . . . . . . . . 76

5 Capillary Absorption of Polymer Droplets $\quad 77$

5.1 Basics of Capillarity . . . . . . . . . . . . . . . . . 78

5.2 Characterization of The Droplet . . . . . . . . . . . . . . 82

5.3 Contact Angle Simulations . . . . . . . . . . . . . . . . . . . . 84

5.4 Capillary Absorption of Polymer Droplets . . . . . . . . . . . . . . . 87

5.4.1 Droplets with 8000 Monomers . . . . . . . . . . . . 87

5.4.2 Droplets with 16000 Monomers . . . . . . . . . . . . . . 94

5.5 Theoretical Analysis . . . . . . . . . . . . . . . . 96

5.6 Conclusions . . . . . . . . . . . . . . . . . . . . 102

6 SPH Simulations of Polymer Blends 103

6.1 Thermodynamics of Phase Separation . . . . . . . . . . . . . . . 104

6.2 Introduction to $\mathrm{SPH} \ldots \ldots \ldots$. . . . . . . . . . . . . . . . . . .

6.3 Application of SPH to Polymer Blends . . . . . . . . . . . . . . . . 114

6.4 Implementation . . . . . . . . . . . . . . . . 118

6.4 .1 Initial Configuration . . . . . . . . . . . . . 118

6.4 .2 Time Integration . . . . . . . . . . . . . . 118

6.4 .3 Linked List . . . . . . . . . . . . . . . . . . . . . . 119 
6.5 Results .............................. 120

6.5.1 Blend with Periodic Boundary Conditions . . . . . . . . . 121

6.5.2 Blend between Two Parallel Plates . . . . . . . . . . . . . . . 124

6.5.3 Blend on a Substrate . . . . . . . . . . . . . . 133

6.5 .4 Blend with Free Surface . . . . . . . . . . . . . . . 137

6.6 Conclusions . . . . . . . . . . . . . . . . . . . . 141

6.6 .1 Future Work . . . . . . . . . . . . . . . . . 142

$\begin{array}{lll}7 & \text { Conclusions } & 144\end{array}$

A Onion-Ring Analysis 


\section{Chapter 1}

\section{Introduction}

Although polymers have been used for centuries, they were not identified as long chain-like molecules until relatively recently. It was Pickles [2] who first suggested that rubber is made up of long chains of molecules. Based on his experimental observations, in 1920 Hermann Staudinger came up with the idea that polymers are molecules made up of covalently bonded elementary units called monomers [3] (for which he received the 1953 Nobel Prize in Chemistry). Given this hypothesis, Paul J. Flory [4] and Maurice L. Huggins [5] (separately) gave the first theoretical treatment of polymer solutions, based on statistical thermodynamics. Flory was awarded the 1974 Nobel Prize in Chemistry for his contributions. In the early 1970s this field of study increased dramatically with the seminal works of Pierre-Giles de Gennes (for which he received the 1991 Nobel Prize in Physics) and Samuel F. Edwards. Here the theoretical treatment of polymers was put on a sound mathematical basis with analogies to other branches of physics (such as quantum mechanics). In fact it was de Gennes who coined the term soft condensed matter physics [6] to describe this new field, which also included other novel condensed matter systems such as liquids crystals, gels, emulsions, colloids and surfactant solutions. Generally, these systems cannot be described (completely) as liquids or solids and thus new theoretical treatments need to be developed to describe them (as opposed to using the well established treatments of liquids or solids). Moreover, since this field is comparatively young it provides a myriad of diverse and interesting problems for the researcher.

\section{Motivation}

With the emergence of nanotechnology, researchers are increasingly interested in polymer-based applications at the nanoscale $[7,8,9,10,11,12,13]$. Nanoscale 
phenomena of polymeric materials are particularly fascinating, because at these dimensions the intrinsic length scales of polymers become comparable with those of device dimensions [10]. Consequently, polymers at the nanoscale are expected to display characteristics that are different from their macroscopic behaviour. The study of polymers at the nanoscale not only bears significance from the viewpoint of their potential applications in nanotechnology, but also presents an exciting challenge to the researcher for deepening our understanding of fundamental science.

In conjunction with theoretical treatments and experiments, simulation studies of polymer systems have also increased dramatically in the last forty years. Owing to their complex molecular structure, and existence of multiple lengthand time-scales, analytical studies of polymers have been difficult $[14,15]$. In this context computer simulations have played an important role in bridging the gap between experiment and analytical theory. Based on the problem at hand and properties of interest, different spatio-temporal scale-regimes become important in polymer systems $[16,17,18]$. This, in turn, determines the level of description required to model these systems: electronic, atomistic, mesoscopic, etc. [16, 19]. In this dissertation we are only concerned with mesoscopic models of polymers, which portray universal properties of polymers very well [15, 18]. Simulation studies of polymers have not only implemented techniques which are already used in condensed matter physics, such as Monte Carlo or Molecular Dynamics (MD), but also sought to introduce new methods, or variants of older methods, which are specific to polymer systems [15].

Our main aim here is to study the behaviour of certain polymer systems at the nanoscale. Due to the wide scope of this thesis, here we shall only give a brief overview of motivation behind each of the problems considered. A much more detailed discussion with sufficient literature review will be given in the respective chapters to follow.

\section{Mixing of Polymeric Fluids in Narrow Channels}

One of the main objectives of micro- or nanofluidic devices is to be able to perform biochemical analysis [20]. Most of these processes demand rapid mixing of polymers/biological molecules [21, 22, 23]. However, flows at these length scales are predominantly laminar and hence mixing is mainly diffusion driven, which is often too slow for these applications [24]. It has been shown that mixing of Newtonian fluids can be enhanced significantly for flows over patterned-slip surfaces $[25,26]$. Using continuum modelling, Pereira [1] studied the effect of patterned- 
slip on the mixing behaviour of non-Newtonian fluids and found some encouraging results. Here we study the mixing behaviour of polymeric fluids over such patterned-slip surfaces using MD simulations and compare the results with the continuum theory predictions of Pereira [1].

\section{Capillary Phenomena of Polymer Droplets}

Applications of capillary phenomena are ubiquitous in industry and daily life. The seminal works of Young and Laplace [27, 28] on the statics of capillarity; and Lucas and Washburn $[29,30]$ on the dynamics of capillarity, form the basis for much of our understanding of this phenomena. These theories describe capillarity on macroscale very well. However, the validity of the Lucas-Washburn approach in the context of nanoscale capillary dynamics has recently been questioned $[31,32,33,34]$.

Another aspect which is worth exploring is the impact of finite size of liquid reservoirs on capillarity, which is usually ignored in standard models. However, such systems are often encountered in nanotechnology where the liquid reservoirs are comparable to channel dimensions; and this can have profound implications on the whole process of capillarity. Thus, in the light of these questions it is even more important to study the effect of finite size reservoirs on capillarity. This question has been investigated for Newtonian fluids and several interesting observations have been made $[35,36,37]$.

However, there have been no such studies so far on polymer droplets. Scientists are interested in producing polymer-based nanorods, nanopatterns [7, 38, 39,40 ] and capillary forces can play an important role here. At the nanoscale, due to the macromolecular nature of their constituents, polymer droplets can exhibit more intriguing capillary phenomena that may not occur in Newtonian fluids. The entropic loss associated with deformation of chains in confined tubes can have complex implications on the process of capillary absorption. In this thesis we investigate the effect of molecular size on the capillary phenomena for small polymer droplets where the chain size of the polymers is comparable with the tube dimensions.

\section{Modelling Freely Deforming Polymer Blends}

One of the more interesting aspects of polymer solutions or polymer blends is the phenomena of self-assembly. For example, binary polymer blends phase separate into domains that are rich in either species. In particular, here we are concerned with the modelling of polymer thin films with freely deformable sur- 
faces, which are of industrial interest $[41,42,43]$. The case of a freely deforming interface is important throughout polymer systems. While conventional particlebased methods can model these free surfaces, in practice, they are problematic since they require a vast number of particles. This leads to numerics which take inordinately long to converge. In the case of self-assembly (especially for deformable free surfaces) continuum methods seem to be much more attractive. The basic problem with continuum treatments is that the basic equations (usually partial differential equations) need to be solved numerically and traditional gridbased solution techniques are implemented. In the case of a freely deformable interface this is a serious issue since the grid on which the equations are solved must be specified before the solution is achieved. In addition to this grid-based methods have several other disadvantages such as anisotropy effects, etc. $[44,45]$.

Another important class of polymeric materials that exhibit complex patterns via self-assembly are block copolymers [46]. In experimental studies of block copolymer systems the liquid melt is usually spin-coated on to solid substrates, forming a thin film of block copolymer melt. In this case a rather strange thing can happen. Rather than remaining flat (as one would expect a normal liquid to do) the free surface can develop undulations [47] or even can form an island and hole structure $[48,49]$. We seek to apply the smoothed particle hydrodynamics (SPH) method to deal with self-assembling polymer systems such as polymer solutions, polymer blends, block copolymer solutions or block copolymer melts. This is a first step in a continuing effort and so we introduce and apply the SPH method to the simplest self-assembling polymer system - a polymer blend. Here we seek to show that the SPH method can be successfully applied to confined and nonconfined polymer blends. We envisage that this method will be extended in the future to other polymer systems, some of which have been elucidated above.

\section{Thesis Outline}

We now briefly outline the content of this thesis. In Chapter 2 we give a basic introduction and background to polymer physics, especially detailing concepts which will be used throughout the remainder of the thesis. In Chapter 3 we give a detailed account of the MD simulation technique which is used for two separate applications later in the thesis. In Chapter 4 we use the MD method to study the mixing of polymeric solutions in narrow microfluidic channels. We compare our MD results with theoretical results and give suggestions for enhancing the mixing in these narrow capillaries. In Chapter 5, we apply the MD method to another microfluidic problem. Here we ask the question - whether a finite polymer droplet can be drawn up a narrow capillary? We find there are some interesting polymer 
attributes which affect the process. In Chapter 6, we present a continuum numerical treatment of the self-assembly of polymer blends specifically focussed on applications where the surfaces can deform. This method depends on not making a spatial discretization of the geometrical domain and has been previously applied to astrophysical and fluid dynamical problems. Finally in Chapter 7 we present Conclusions for our work. 


\section{Chapter 2}

\section{Polymer Physics}

Polymers are large molecules composed of a sequence of many small molecules that are connected to each other by covalent bonds. The basic repeating unit of a polymer is called a monomer. A monomer in itself can consist of one or more different chemical entities. The concepts of polymer and monomer are illustrated in Fig. 2.1 for a polyethylene molecule.

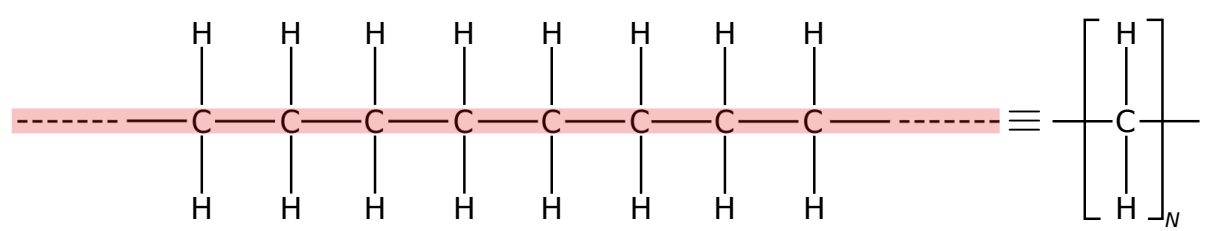

Figure 2.1: A polyethylene molecule with $\mathrm{N}-\mathrm{CH}_{2}$ monomeric units. The shorthand notation on the right describes the molecule in terms of its monomeric unit. The red shaded region in the figure indicates the chain backbone which runs along the chain connecting all monomer centres.

The number of monomer units in a polymer chain is called the polymerization index $(N)$ of the chain. In some cases it is often useful to redefine the monomer as a group of basic monomers to reduce the complexity and the resulting representative polymer is said to be coarse-grained [15]. Given their complex molecular architecture, which is a characteristic of polymers, it is rather difficult to construct atomistic models that include all degrees of freedom. Fortunately, many polymer systems exhibit universal behaviour independent of details on the molecular scale. This grants us the freedom to build coarse-grained models that capture the essential behaviour of polymers and such modelling has proven to be very useful $[15,17]$. A detailed discussion on coarse-graining will be provided in the next chapter. 
The theoretical framework of polymer systems has evolved towards exploiting the universality aspect of these materials. In the following sections, we discuss some of the basic concepts in polymer physics which are essential to understand the content of this dissertation. Our understanding of these concepts comes mainly from sources $[50,51,46,52,53]$.

\subsection{Basic Concepts in Polymer Physics}

Based on the chemical composition and arrangement of constituents, polymers can be categorized into either homopolymers or heteropolymers. Polymers that constitute only one type of monomer are known as homopolymers. They are further divided into different groups depending on the molecular architecture. For example, linear, star, branched, etc. as shown in Fig. 2.2. Polymer molecules that consist of more than one type of monomer species are known as heteropolymers or copolymers. A wide range of copolymers can be realized by varying the molecular arrangement and chain composition (for example see Fig. 2.2).
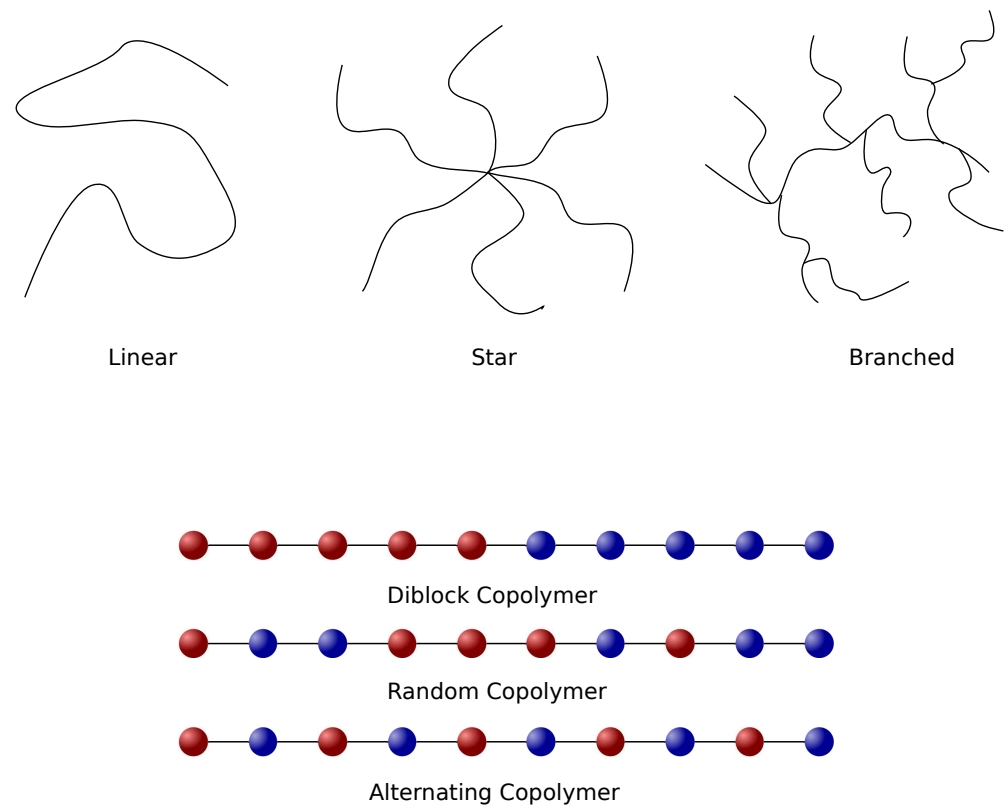

Figure 2.2: The upper half of the figure displays some example homopolymers. Here polymers are represented as continuous chains. The lower half of the figure displays some example (linear) copolymers, where the red and blue colours are used to differentiate two types of monomers. The black line between any two consecutive monomers is the bond connecting them.

\section{Flexibility}

One of the most important properties used to characterize polymers is their flexibility. It has a significant impact on both static and dynamic properties of 
polymers such as elastic moduli [54] and conformational dynamics [55], etc. The flexibility of a chain depends on the information regarding its bond lengths, bond angles and torsion angles. The bond length is the distance between any two consecutive monomers. The angle between any two consecutive bonds (three consecutive monomers) is defined as the bond angle. The last and most important (as we will see) is the torsion angle which largely determines the flexibility of polymers [46]. It is defined as the angle formed between two planes, where the first plane is constructed by joining the first three monomers of the quadruplet (a group of four consecutive monomers) and the second plane is constructed by joining the last three monomers of the same quadruplet. The torsion angle is pictorially demonstrated in Fig. 2.3(a).

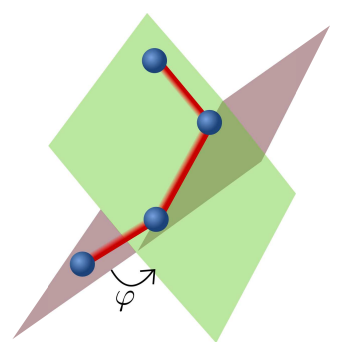

(a)

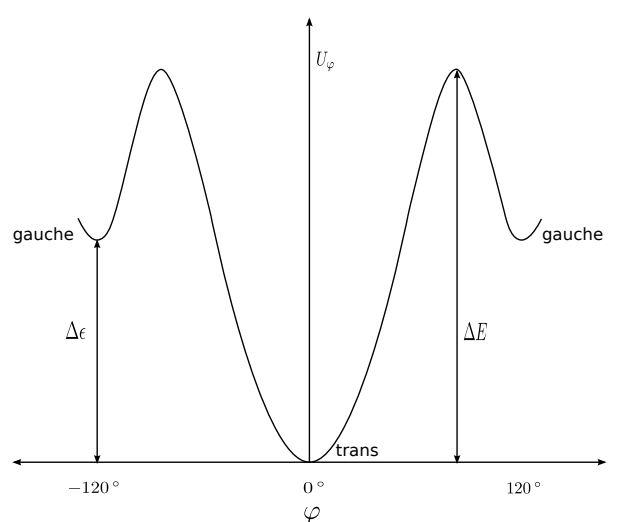

(b)

Figure 2.3: (a) A part of a polymer chain is shown here. The (blue) monomers are connected to each other by (red) bonds. The angle between the two intersecting planes gives us the torsional angle $\varphi$. (b) The energy variation as a function of $\varphi$ (see text).

In most polymers, fluctuations in the torsion angles determine the flexibility, while the fluctuations in bond lengths and bond angles are often too small to have any significant effect. The typical variation in energy as a function of torsion angle is shown in Fig. 2.3(b). The torsion angles corresponding to the three minima, from left to right in Fig. 2.3(b), are called gauche-, trans and gauche ${ }^{+}$conformations, respectively. The reason behind this energy variation upon a change in $\varphi$ can be understood in the following way. At the molecular level, a change in $\varphi$ results in a change in distance between various constituents (atoms) of different monomers, which, in turn, gives rise to a change in energy.

If a chain is in the all trans state then it is fully stretched to its maximum length. The ratio of trans to gauche states determines the flexibility of a chain. Of course, the occupancy of trans or gauche states depends on the temperature 
of the system. If $k_{B} T>\Delta \epsilon$ i.e., the thermal energy is greater than the energy difference between trans and gauche states then the chain is essentially flexible. As the temperature decreases, $\Delta \epsilon / k_{B} T$ increases, and trans states become more preferential. Thus the chain starts to behave more rigidly on the local scale. But as we zoom out, above a certain critical segment length the chain appears to be flexible again. The segment length below which the chain appears to be rigid, and above which it appears to be flexible is known as the persistence length of the chain. Note, for some polymers the persistence length can be greater than their chain length. In such a scenario, the chain is rigid at all length scales.

\subsection{Ideal Chain Models}

The configurational space of a given polymer chain depends on its flexibility and its interactions with the surroundings. Different models have been proposed to study different types of polymers based on their flexibility [50,46]. Here we are only concerned with flexible polymers.

In an ideal chain model, hard-core repulsive interactions, that prevent any two monomers from occupying the same space, are completely ignored. Ideal chain models account for short-range interactions while ignoring long-range interactions altogether. In the present context, the terms short- and long-range hold different meanings to their conventional use. The interaction between any two monomers that are separated by a small number of monomers along the chain backbone is considered to be short-ranged while between those separated by a large number of monomers is considered to be long-ranged. The fact that two monomers separated by a large distance along the chain backbone can still come close in space is completely ignored in all ideal chain models. But as we will see, despite this approximation ideal chain models can replicate the generic behaviour of flexible polymers. In this section we shall discuss some ideal chain models of flexible polymer chains such as the freely jointed chain model, freely rotating chain model, and the Gaussian chain model.

\subsubsection{Freely Jointed Chain (FJC) Model}

In this model, the orientation of a bond is considered to be independent of any other bond in the chain including its immediate predecessor. That is, a bond is free to take any orientation it likes. The bond length is assumed to remain constant while torsion angles are ignored. Since the orientation of a bond is independent of any other, there is no correlation between any two bonds. Mathematically, this model is equivalent to a random walk. 


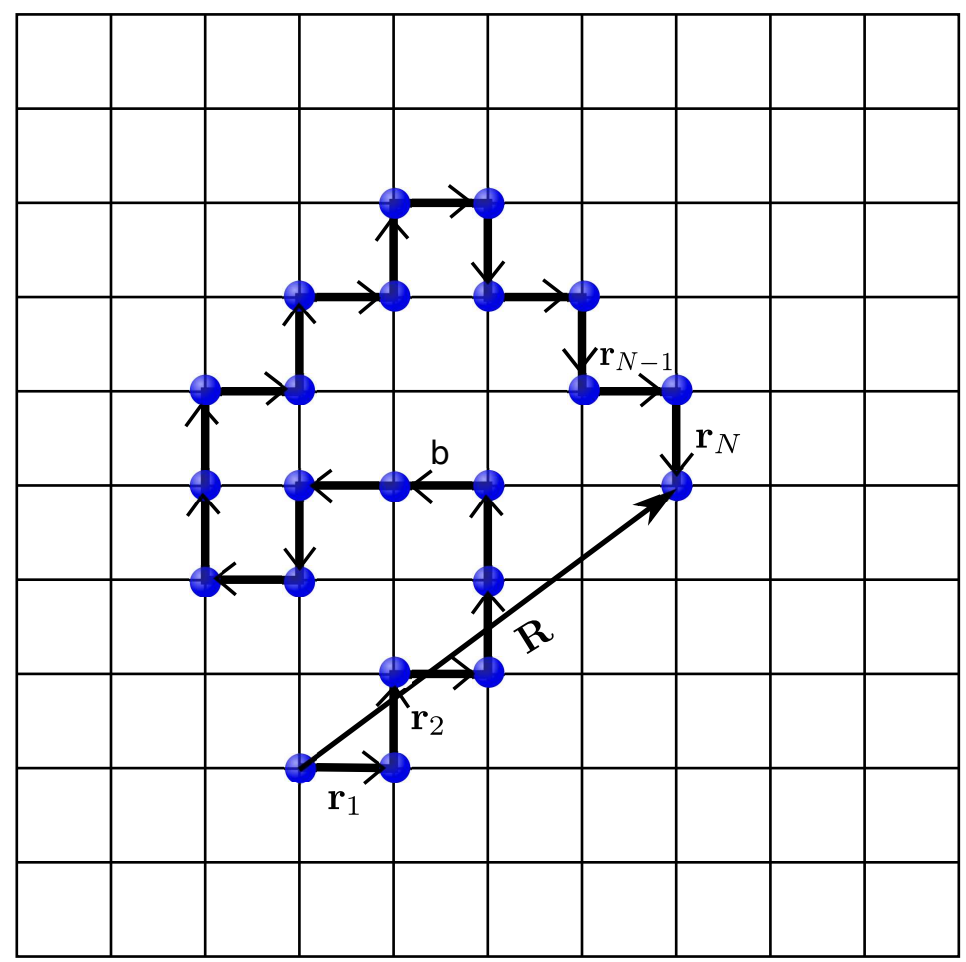

Figure 2.4: A schematic diagram showing a FJC chain on a lattice.

Now let us consider a polymer chain with $N$ segments (bonds), each with bond length $b$ on a lattice as shown in Fig. 2.4. One of the important properties used to characterize polymers is their size. To calculate this, let us try to compute the end-to-end vector $\mathbf{R}$. If the bond vectors representing the chain are denoted as $\mathbf{r}_{1}, \mathbf{r}_{2}, \cdots, \mathbf{r}_{N}$, where $\mathbf{r}_{i}$ is the bond vector connecting monomer $i$ to $i+1$, then

$$
\mathbf{R}=\mathbf{r}_{1}+\mathbf{r}_{2}+\cdots+\mathbf{r}_{N}
$$

In the FJC model, the ensemble average $\langle\mathbf{R}\rangle=0$ since each bond is likely to span all possible orientations. Obviously $\langle\mathbf{R}\rangle$ is not a good measure of chain dimensions. The second moment of $\mathbf{R}$, that is the mean square end-to-end distance is a better choice for polymer size and it is calculated as

$$
\begin{aligned}
\langle\mathbf{R} \cdot \mathbf{R}\rangle & =\left\langle\left(\sum_{i=1}^{N} \mathbf{r}_{i}\right) \cdot\left(\sum_{j=1}^{N} \mathbf{r}_{j}\right)\right\rangle \\
& =\left\langle\sum_{i, j=1}^{N} \mathbf{r}_{i} \cdot \mathbf{r}_{j}\right\rangle \\
& =\left\langle\sum_{i=1}^{N} \mathbf{r}_{i}^{2}\right\rangle+\left\langle\sum_{i \neq j} \mathbf{r}_{i} \cdot \mathbf{r}_{j}\right\rangle
\end{aligned}
$$




$$
\begin{aligned}
& =N b^{2}+\left\langle\sum_{i \neq j}\left|\mathbf{r}_{i}\right|\left|\mathbf{r}_{j}\right| \cos \theta_{i j}\right\rangle \\
\left\langle\mathbf{R}^{2}\right\rangle & =N b^{2}+b^{2}\left\langle\sum_{i \neq j} \cos \theta_{i j}\right\rangle .
\end{aligned}
$$

The second term in Eq. (2.6) is as in a random chain there are no correlations between any two distinct bonds. Therefore,

$$
\left\langle\mathbf{R}^{2}\right\rangle=N b^{2}
$$

The disadvantage with the FJC model is that it does not prevent a chain segment from folding back onto its predecessor, which is clearly unphysical. By incorporating correlations between any two distinct segments we can rectify this deficiency. The mean square end-to-end vector with short-range interactions in place takes the following form

$$
\begin{aligned}
\left\langle\mathbf{R}^{2}\right\rangle & =b^{2} \sum_{i=1}^{N} \sum_{j=1}^{N}\left\langle\cos \theta_{i j}\right\rangle \\
& =C_{N} N b^{2}
\end{aligned}
$$

where $C_{N}=\sum_{i=1}^{N}\left\langle\cos \theta_{i j}\right\rangle / N$ is Flory's characteristic ratio. Note that, even with short-range interactions included the relationship $\left\langle R^{2}\right\rangle \propto N b^{2}$ still holds.

One of the advantages with the FJC model is that all ideal chains can be reduced to a FJC. In the FJC model, the short-range interactions between monomers enter only via the bond length $b$ and there are no correlations between any two bonds. Any ideal chain with a bond length $b$ can be represented by an equivalent FJC with a new renormalized bond length $b_{K}$, chosen such that there are no correlations between any two bonds. The renormalized segment length $b_{K}$ is known as the Kuhn statistical length of the chain. The magnitude of $b_{K}$ indicates the stiffness of a chain. A small $b_{K}$ means the chain is flexible and a large $b_{K}$ means the chain is rigid, globally. In other words, the Kuhn statistical length describes the minimum distance between two points on a polymer chain that are essentially uncorrelated. Hence, any ideal chain with bond length $b_{K}$ is equivalent to a FJC with same bond length.

Since a FJC is equivalent to a random walk, the probability distribution of a chain with $N$ segments as a function of end-to-end vector $\mathbf{R}$ in three dimensions 
is given by

$$
\Phi(\mathbf{R}, N)=\left(\frac{3}{2 \pi N b^{2}}\right)^{3 / 2} \exp \left(-\frac{3 R^{2}}{2 N b^{2}}\right) .
$$

Therefore, a FJC has a Gaussian distribution for the end-to-end vector. Using this probability distribution function we can compute some important thermodynamic quantities such as the entropy and the Helmholtz energy. The entropy

$$
S(\mathbf{R}, N)=k_{B} \ln \Omega_{N}(\mathbf{R}, N)
$$

where $\Omega(\mathbf{R}, N)$ is the number of conformations that start at origin $O$ and end at $\mathbf{R}$ in $N$ steps and it is directly proportional to the probability for such a conformation/walk. From Eqs. (2.9) and (2.10), we get

$$
S(\mathbf{R}, N)=S_{0}-\frac{3 \mathbf{R}^{2}}{2 N b^{2}}
$$

where $S_{0}$ is a constant. From Eq. (2.11), with increasing $\mathbf{R}$, the entropy decreases. The Helmholtz energy

$$
\begin{aligned}
& F(\mathbf{R}, N)=U-T S(\mathbf{R}, N) \\
& F(\mathbf{R}, N)=F_{0}+\frac{3 K_{B} T \mathbf{R}^{2}}{2 N b^{2}}
\end{aligned}
$$

where $F_{0}$ is a constant. Note the $R^{2}$ dependency of the Helmholtz energy, this indicates that an ideal chain behaves like a "Hookean spring".

\subsubsection{Freely Rotating Chain (FRC) Model}

In this model all bond lengths and bond angles remain constant. The difference from the FJC model lies in including the correlations between monomers by means of torsional angles. The torsional angles are free to assume any value between $-\pi<\varphi<\pi$, thus bonds are free to rotate. This is the source of flexibility in FRC models. This model is illustrated with the help of Fig. 2.5.

Let us focus on the bond vector $\mathbf{r}_{j+1}$ in Fig. 2.5. For simplicity, assume that all other bond vectors are frozen except for $\mathbf{r}_{j+1}$. This bond vector is free to rotate about $\mathbf{r}_{j}$. To compute bond vector $\mathbf{r}_{j+1}$ 's correlation with other vectors we must compute its components along the chain backbone. The normal component of $\mathbf{r}_{j+1}$ along $\mathbf{r}_{j}$ is $b \sin \theta$. Since the bond vector is free to rotate, the ensemble average of this quantity is equal to zero, i.e., $b\langle\sin \theta\rangle=0$. Hence there is no correlation due to the normal component. However, the longitudinal component of $\mathbf{r}_{j+1}$ along $\mathbf{r}_{j}$ is equal to $b \cos \theta$, which does not vanish in the ensemble average. 


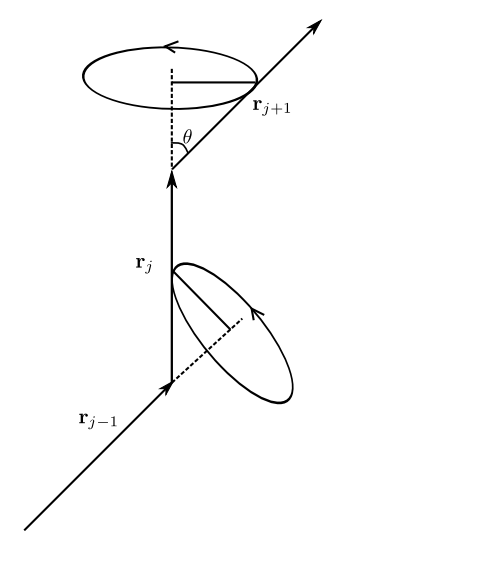

Figure 2.5: Portion of a freely rotating chain is shown here. $\theta$ is the bond angle between any consecutive monomers. The circles represent the free rotation of bond vectors about their predecessors.

Using the same argument, the longitudinal component of $\mathbf{r}_{j+1}$ along $\mathbf{r}_{j-1}$ is equal to $b \cos ^{2} \theta$ and so on. In this manner the correlation of a bond vector is transmitted through the chain backbone in the form of its longitudinal component. Generalizing the above approach, for any two bond vectors $i$ and $j$

$$
\left\langle\mathbf{r}_{i} \cdot \mathbf{r}_{j}\right\rangle=b^{2}(\cos \theta)^{|i-j|} .
$$

As it can be inferred from Eq. (2.14), the correlation between any two bond vectors decreases with increasing distance between them along the chain contour. From Eqs. (2.3) and (2.14), after some algebra we arrive at

$$
\left\langle\mathbf{R}^{2}\right\rangle=N b^{2} \frac{1+\cos \theta}{1-\cos \theta} .
$$

Once again note that the end-to-end distance satisfies the relationship $\left\langle R^{2}\right\rangle \propto$ $N b^{2}$. Using scaling arguments it can be shown that the distribution function for $\mathbf{R}$ of a FRC has the same form as that of a FJC.

\subsubsection{The Gaussian Chain Model}

In this model the chain is divided into a number of equal segments. The segment length $b$ is chosen such that there are no correlations between any two segments. All segments are assumed to be flexible and satisfying the Gaussian distribution:

$$
\psi(\mathbf{R}, N)=\left(\frac{3}{2 \pi b^{2}}\right)^{3 / 2} \exp \left(-\frac{3 R^{2}}{2 b^{2}}\right)
$$


As there are no correlations between segments, the total chain conformation is simply the product of individual segment conformations. For a $N$ segment chain, this quantity is given by

$$
\begin{aligned}
\Psi\left(\left\{\mathbf{r}_{N}\right\}\right) & =\prod_{i=1}^{N} \psi\left(\mathbf{r}_{i}\right) \\
& =\left(\frac{3}{2 \pi b^{2}}\right)^{3 N / 2} \prod_{i=1}^{N} \exp \left(-\frac{3 \mathbf{r}_{i}^{2}}{2 b^{2}}\right) \\
\Psi\left(\left\{\mathbf{r}_{N}\right\}\right) & =\left(\frac{3}{2 \pi b^{2}}\right)^{3 N / 2} \exp \left(-\sum_{i=1}^{N} \frac{3\left(\mathbf{R}_{i}-\mathbf{R}_{i-1}\right)^{2}}{2 b^{2}}\right),
\end{aligned}
$$

where $\left\{\mathbf{r}_{N}\right\} \equiv\left(\mathbf{r}_{1}, \mathbf{r}_{2}, \cdots, \mathbf{r}_{N}\right)$ is the conformational state of the chain and $\mathbf{r}_{n}=$ $\mathbf{R}_{n}-\mathbf{R}_{n-1}$. Here $\mathbf{r}_{i}$ and $\mathbf{R}_{i}$ are the bond- and position-vectors of segment $i$, respectively. We can rewrite Eq. (2.19) as

$$
\Psi\left(\left\{\mathbf{r}_{N}\right\}\right)=\left(\frac{3}{2 \pi b^{2}}\right)^{3 N / 2} \exp \left(-\frac{U_{0}\left(\left\{\mathbf{r}_{N}\right\}\right)}{k_{B} T}\right)
$$

where

$$
U_{0}\left(\left\{\mathbf{r}_{N}\right\}\right)=\frac{3 k_{B} T}{2 b^{2}} \sum_{i=1}^{N}\left(\mathbf{R}_{i}-\mathbf{R}_{i-1}\right)^{2}
$$

From Eqs. (2.20) and (2.21), it can be inferred that a Gaussian chain is equivalent to a chain where consecutive monomers are connected to each other via springlike bonds. Any portion of the chain, between two segments $n$ and $m$ also behaves as a Gaussian chain with distribution function

$$
\Phi\left(\mathbf{R}_{n}-\mathbf{R}_{m}, n-m\right)=\left(\frac{3}{2 \pi|n-m| b^{2}}\right)^{3 / 2} \exp \left[-\frac{3\left(\mathbf{R}_{n}-\mathbf{R}_{m}\right)^{2}}{2|n-m| b^{2}}\right]
$$

\subsubsection{Limitations of Ideal Chain Models}

As mentioned earlier, ideal chain models fail to account for long-range interactions. Such interactions are present in real polymers. How important these interactions are for a given polymer depends on the number of monomer-monomer contacts. To understand this let us introduce the following concepts:

- Pervaded volume: It is defined as the volume spanned by a polymer in solution

$$
V \approx R^{d}
$$


where $R$ is the size of the chain and $d$ is the dimensionality of the system.

- Overlap volume fraction: It is defined as the volume fraction of the pervaded volume that is actually occupied by the chain and is given by

$$
\phi^{*} \approx \frac{N b^{d}}{R^{d}}
$$

where $b$ is the bond length.

For an ideal chain, $R \approx \sqrt{N b^{2}}$ and substituting this into the Eq. (2.24), we get $\phi^{*} \approx N^{1-d / 2}$. The number of monomer-monomer contacts is equal to the product of the chain length and the overlap volume fraction, $N \phi^{*}$. This also includes the contacts between monomers that are separated by large number of monomers along the chain contour. From Eq. (2.24), $N \phi^{*}=N^{2-d / 2}$. In three dimensions this quantity is equal to $N^{1 / 2}$ which is a large number for long ideal chains. That is, the number of monomer-monomer contacts is significant for long chains with high overlap volume fractions. Ideal chain models are flawed in this limit.

\subsection{Real Polymers}

\subsubsection{Excluded Volume Effect}

In real polymers, when two monomers approach each other in space the steric interactions prevent them from overlapping and this effect is known as the excluded volume effect. The steric interaction between two monomers is similar to that between two inert atoms. At short distances there is a strong repulsion due to Pauli's exclusion principle and at large distances there is a weak attraction originating from dipole-dipole interactions. The basic form of this interaction between two monomers is plotted in Fig. 2.6.

In addition to monomer-monomer interactions there can be monomer-solvent interactions present in the system. The form of the attractive tail in Fig. 2.6 depends on the relative difference between these two competing forces. The tradeoff between these two forces determines whether monomer-monomer contacts are more preferred over monomer-solvent contacts or the other way around. 


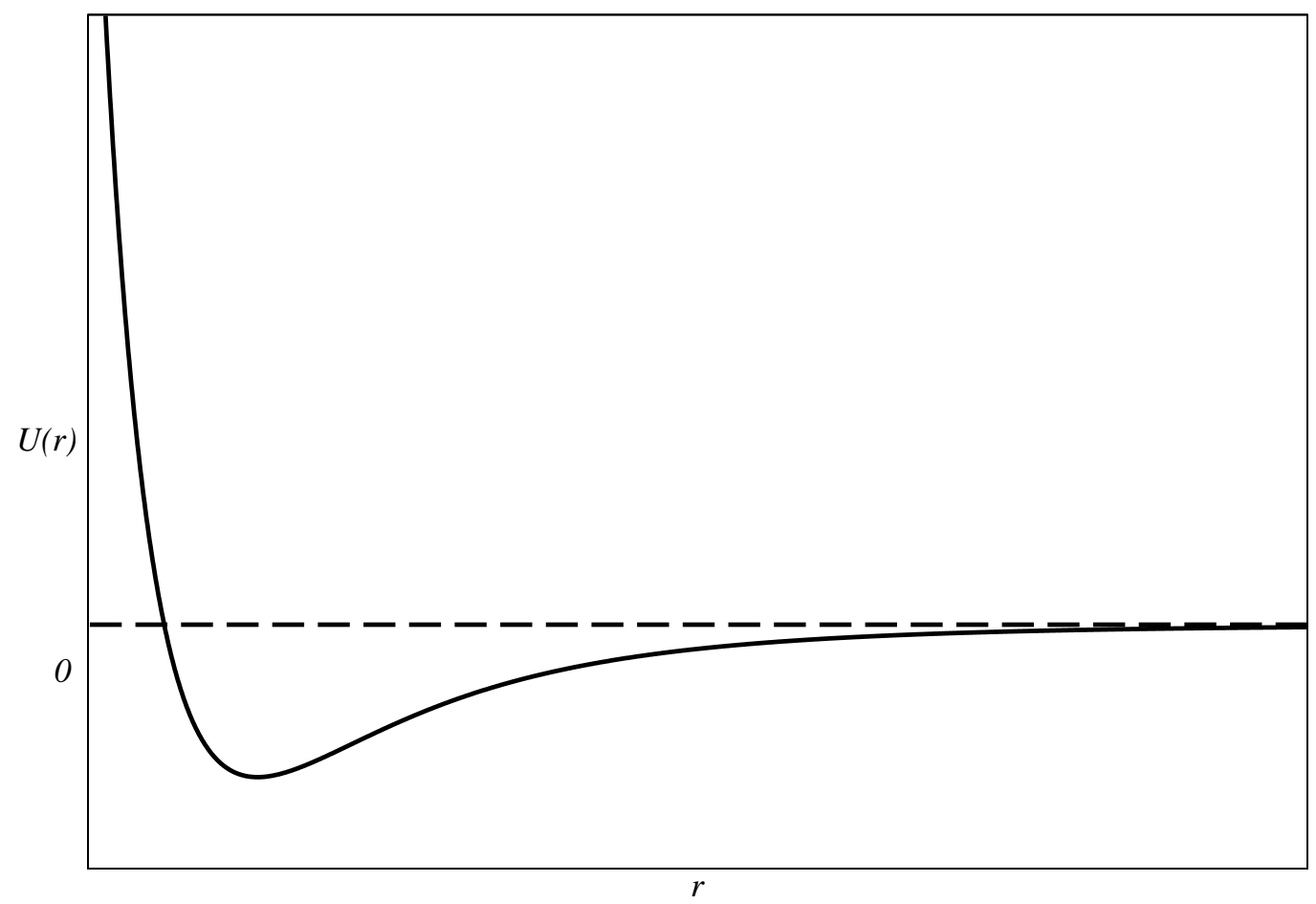

Figure 2.6: Potential energy between two monomers as a function of distance $r$.

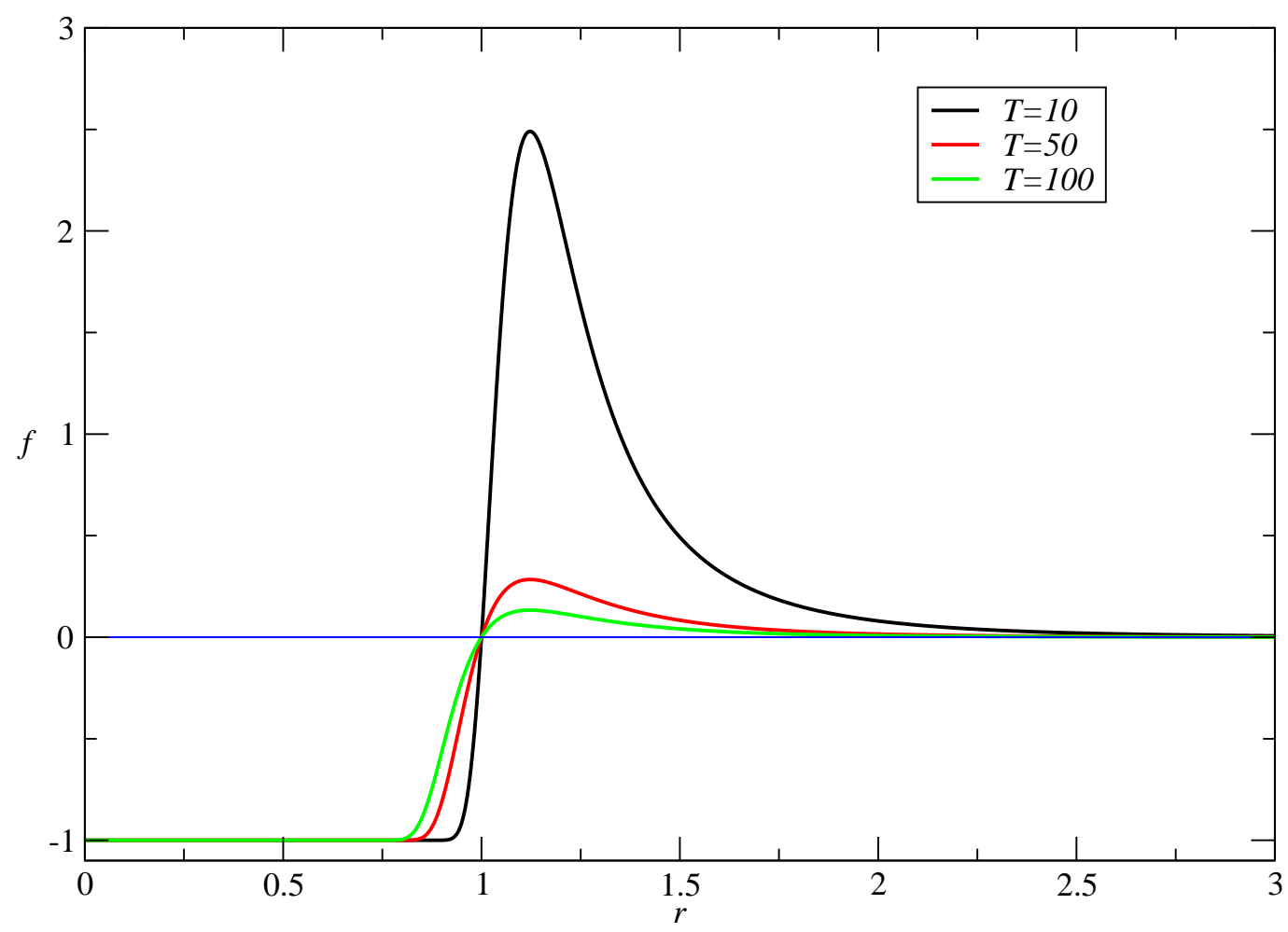

Figure 2.7: Meyer's function at different temperatures. 
The probability of finding two monomers $r$ distance apart is proportional to the Boltzmann factor $\exp \left[-U(r) / k_{B} T\right]$, where $U(r)$ is the energy of interaction between the monomers. As expected, the probability of finding another monomer close to the centre of reference monomer is diminishingly small and increases sharply close to the radius of the monomer. The probability slowly decreases away from this point and attains a constant value 1 at long distances. The Mayer's function $f$ defined as $\exp \left[-U(r) / k_{B} T\right]-1$ is used to compute the excluded volume. It is plotted in Fig. 2.7 for different temperatures $\left(k_{B}=1\right)$.

At a particular $T$, the excluded volume is defined as the area under the curve $-f$, that is $v=-\int f d \mathbf{r}$. In the high $T$ limit, the excluded volume $v$ has a large positive value and becomes independent of the temperature. Owing to this the system is referred to as athermal solvent. The reason for this is that a specimen monomer does not distinguish between another monomer or a solvent particle as it just experiences the hard-core repulsion. If the monomer-solvent attraction dominates the monomer-monomer attraction then it leads to a small positive value of $v$ and the polymer is said to be in good solvent conditions. As the temperature increases, at some particular value $\Theta$ both the repulsive and attractive contributions to the excluded volume cancel out each other. Thus, the chain acts as an ideal chain at $\Theta$-temperature. For $T<\Theta$, the attractive part of the integral dominates the repulsive part and hence monomers like to come close to each other. This behaviour occurs under poor solvent conditions.

This means that, depending on the temperature, polymer chains behave differently. Under the three different temperature domains discussed above the size of the polymers vary significantly. In good solvent conditions the polymer chain likes the solvent particles and thus its size increases compared to an ideal chain of same length. On the contrary, in a poor solvent monomers come close and form a coiled conformation. Interesting behaviour appears near the $\Theta$-temperature where the excluded volume becomes zero and the chain behaves as an ideal chain.

\subsubsection{Probability Distribution for $R$}

A real polymer chain with excluded volume interactions can be thought of as a Self Avoiding Random Walk ( $S A W)$. In a SAW, the next segment of the chain can only proceed to a site which is not being occupied already. Such a chain is known as a non-Markovian chain in that it has memory of sites it has already visited.

To calculate the probability distribution function for $\mathbf{R}$, let us consider the total number of walks starting at the origin $O$ and ending between $\mathbf{R}$ and $\mathbf{R}+d \mathbf{R}$ 
and denote it by $W_{0}(R) d R$. This includes both random walks and SAWs. For a chain with $N$ segments on a lattice with coordination number $z$, the total number of such walks that have an end-to-end distance $R$ is

$$
W_{0}(\{R\})=z^{N}
$$

Out of these $z^{N}$ conformations the probability for a chain with $N$ segments to have an end-to-end distance between $R$ and $R+d R$ is

$$
W_{0}(R)=z^{N} 4 \pi R^{2} d R\left(\frac{3}{2 \pi N b^{2}}\right)^{3 / 2} \exp \left(-\frac{3 R^{2}}{2 N b^{2}}\right) .
$$

Eq. (2.26) includes all possible walks of $N$ steps. By eliminating all the random walks from $W_{0}(R)$, we can obtain the number of SAWs with end-to-end distance $R$.

For this purpose we use the excluded volume interactions. If $v_{c}$ is the excluded volume of each monomer, assuming the chain is homogeneously spread in space, the probability for a site being occupied by a monomer is $v_{C} / R^{3}$. Then, the probability for a site not being occupied by a monomer is $1-v_{c} / R^{3}$. Two monomers occupying a single site amounts to two segments overlapping. Since the number of distinct pairs for a chain with $N$ segments is $N(N-1) / 2$, the probability of finding a walk with no-overlaps is

$$
\begin{aligned}
P_{e x}(R) & =\left(1-\frac{v_{C}}{R^{3}}\right)^{N(N-1) / 2} \\
& =\exp \left[\frac{N(N-1)}{2} \log \left(1-\frac{v_{c}}{R^{3}}\right)\right] .
\end{aligned}
$$

For $N>>1$ and $v_{c} / R^{3}<<1$, the above equation reduces to

$$
P_{e x}(R)=\exp \left(-\frac{v_{c} N^{2}}{2 R^{3}}\right)
$$

Out of all possible walks given by Eq. (2.26), the number of walks that satisfy the excluded volume condition in Eq. (2.28) is

$$
\begin{aligned}
W(R) & =W_{0}(R) P_{e x}(R) \\
& =z^{N} 4 \pi R^{2} d R\left(\frac{3}{2 \pi N b^{2}}\right)^{3 / 2} \exp \left(-\frac{3 R^{2}}{2 N b^{2}}-\frac{v_{c} N^{2}}{2 R^{3}}\right) .
\end{aligned}
$$

The first term within the exponent argument in Eq. (2.29) is the entropic con- 
tribution and the second term is due to the energetic contribution. To find the equilibrium end-to-end distance we have to maximize Eq. (2.29) with respect to $R$ and this gives us

$$
R \simeq R_{0}\left(\frac{N^{1 / 2} v_{c}}{b^{3}}\right)^{1 / 5}
$$

where $R_{0}$ is the end-to-end distance for an ideal chain of the same length. The chain dimensions increase significantly for a SAW chain $\left(R \propto N^{3 / 5}\right)$ compared to an ideal chain $\left(R \propto N^{1 / 2}\right)$. This effect is known as swelling of chains.

\subsubsection{A Polymer Chain in a Melt is Ideal}

Based on the concentration of polymers in solution, polymer liquids are classified as dilute, semi-dilute or polymer melt. In a dilute solution the concentration of polymers is low and there are no overlaps between different chains. As the polymer concentration increases, above a certain critical concentration chains invade pervaded volumes of other chains. This results in significant overlaps and the system is said to be in semi-dilute conditions. If the system is densely packed with polymer molecules only without any solvent then it is referred to as a polymer melt. We limit our discussion to this case only.

In a polymer melt, the system is densely packed with polymer molecules. In such a scenario, how does an individual chain behave? Using self-consistent mean field argument, each chain is surrounded by approximately equal number of other chains. Therefore, each monomer of a representative chain experiences the same amount of attraction and repulsion from its surroundings . Hence, on average the chain experiences no net interaction. Therefore, a chain in a melt is ideal. This situation is analogous to the $\Theta$-temperature case, and hence the melt is often referred to as $\Theta-$ solvent.

\subsection{Radius of Gyration}

The mean square end-to-end vector is not always the most useful quantity for describing non-linear polymers, for example, branched polymers, ring polymers, etc [46]. The most commonly used quantity for measuring the size of polymers is the mean square radius of gyration. Moreover this quantity can be directly measured from light scattering experiments. Therefore, the radius of gyration $\left(R_{g}\right)$ allows us to test theoretical predictions against experimental results.

The mean square radius of gyration is defined as the average mean square 
distance of each monomer from the centre of mass of the chain

$$
\left\langle R_{g}^{2}\right\rangle=\frac{1}{N}\left\langle\sum_{i=1}^{N}\left(\mathbf{r}_{i}-\mathbf{R}_{c m}\right)^{2}\right\rangle,
$$

where \langle\rangle denotes ensemble average and

$$
\mathbf{R}_{c m}=\frac{1}{N} \sum_{i=1}^{N} \mathbf{r}_{i},
$$

is the centre of mass position of the chain. For an ideal chain the following relationship between the mean square radius of gyration and mean square end-toend distance holds

$$
\left\langle R_{g}^{2}\right\rangle=\frac{\left\langle R^{2}\right\rangle}{6}
$$

\subsection{Viscoelasticity}

Polymer melts are viscoelastic materials, which means that when a stress is applied they exhibit both solid-like (elastic) and fluid-like (viscous) behaviour. This property is known as viscoelasticity. To elucidate this property we consider a Hookean solid and a Newtonian fluid. The stress response of a Hookean solid is given by:

$$
\underline{\underline{\tau}}=-G \gamma
$$

where $\underline{\underline{\tau}}$ is the stress, $G$ is the elastic modulus and $\gamma$ is the strain in the solid. Similarly, the stress response of a Newtonian fluid is given by

$$
\underline{\underline{\tau}}=-\eta \dot{\gamma}
$$

where $\eta$ is the viscosity of the fluid and $\dot{\gamma}$ is the shear rate.

Polymers exhibit both these properties. The simplest model which represents viscoelasticity of polymers is the Maxwell model [56], which can be imagined as a Hookean spring connected in series with a viscous dashpot. In such a series combination, the stress on both elements is the same while the strain experienced by the material is the sum of strains on each element. Using these concepts, differentiating Eq. (2.34) and adding it to Eq. (2.35), we arrive at the Maxwell constitutive equation for viscoelastic fluids:

$$
\underline{\underline{\tau}}+\Lambda \frac{\partial \underline{\underline{\tau}}}{\partial t}=-\eta \dot{\gamma}
$$


where $\Lambda=\eta / G$ is the relaxation time, up to which point the material behaves like a solid and above which it flows as a fluid. However, unlike in the above model, many polymers do not relax in a single relaxation time, i.e. they exhibit multiple relaxation times [56]. 


\section{Chapter 3}

\section{Molecular Dynamics}

Statistical mechanics is an important branch of theoretical physics. It connects the microscopic details of the system such as molecular masses, positions and velocities to macroscopically observable quantities such as density, temperature, pressure, etc [57]. Only a handful of problems in statistical mechanics can be solved analytically. Most often complexity of the problem has to be simplified in a theoretical model in order to obtain analytical solutions. Such a heuristic approach does not yield useful results all the time [58]. As a consequence, a direct comparison between theory and experiment is not always possible.

Computer simulations have played a prominent role in filling the gap between the theories and experiments [59]. Using computer simulations, in principle, theoretical models can be implemented without making any further approximations. However, in practice some approximations are still made to improve the efficiency of the algorithms. Despite these approximations, simulations can offer more realistic models of experimental systems than theoretical methods in most cases. Results obtained from simulations can be compared with both theoretical predictions and experimental results. A good agreement between theory and simulation can validate the accuracy of the theory in explaining the underlying phenomena. Similarly, a comparison between simulation and experiment can validate the accuracy of the model.

Computer simulations also provide us with a framework for carrying out new pseudo experiments i.e., subjecting the system under study to a new environment or predicting the behaviour of a new system. For example, simulations can be made of a system under extreme temperature or pressure [58]. Conducting such experiments in reality may be impractical and/or expensive. In experiments, there is a great emphasis on the sample preparation under the right conditions. This 
process is very difficult to control and as a result samples are prone to defects and impurities. However this is not an issue in simulations. Computer simulations grant us a great degree of control over the sample preparation process and the sample can be as perfect as desired. Simulations have also become a testing tool for experimental setups [60]. Today, many experimental and theoretical studies are complemented with simulations.

Molecular dynamics [59] and Monte Carlo [59] methods are two of the most commonly used simulation techniques that are available for studying properties of materials. Molecular dynamics is a deterministic method. In this method, particle positions are determined from force field calculations thus allowing dynamic evolution of the system to be followed. As a result, molecular dynamics is the ideal choice for studying dynamic properties such as viscosity, diffusion coefficient, etc. $[59,61,62]$. On the other hand, the Monte Carlo method is based on exploring the energy surface by random sampling and is mainly designed to study equilibrium properties. We have used molecular dynamics extensively in this thesis work and shall restrict our discussion to this method only.

Molecular dynamics (MD) is a particle based simulation technique used for studying equilibrium and dynamic properties in a wide range of problems, starting from nonequilibrium properties of liquids, defects in crystals, nanoclusters, biomolecules, electronic properties of materials, etc [58]. It essentially involves solving Newton's equation of motion for a system of particles on a computer, allowing us to compute properties of interest through a representative time average.

MD is capable of providing detailed information about the system under investigation which might not be possible to obtain from experiments [61]. Information on positions and velocities of the individual particles is difficult to retrieve from real experiments whereas these data are readily available for analysis in a MD simulation at all times. In experiments, the calculated physical quantities are averages over the period of measurement and number of particles [61]. Molecular dynamics provides us with instantaneous snapshots of the particle positions and velocities which in turn are used to calculate various properties of interest either by evaluating them during the course of the simulation or by storing the information on positions and velocities for post-simulation analysis. In this manner, MD relates microscopic details such as positions and velocities to macroscopic properties such as temperature, energy etc. albeit via the laws of statistical mechanics. 


\subsection{Connection to Statistical Mechanics}

In a classical system consisting of few particles, physical properties can be obtained by solving equations of motion. Such an approach is highly impractical as the system size grows and becomes impossible to apply to macroscopic systems $\left(\approx 10^{23}\right.$ atoms). Fortunately, statistical mechanics provides an alternative approach to study physical properties of macroscopic systems. In statistical mechanics, macroscopic properties of interest are extracted from microscopic details via distribution functions that describe the probability of finding the system in a particular microscopic state. A microscopic state or simply a state is the description of positions and velocities of all particles in the system. A collection of such microstates which obey the same macroscopic properties is called an "ensemble". The observables are then calculated as ensemble averages i.e., an average over all microstates. For example, for a system of $N$ particles, with position coordinates $\mathbf{r}_{1}, \mathbf{r}_{2}, \cdots, \mathbf{r}_{N}$ and momenta coordinates $\mathbf{p}_{1}, \mathbf{p}_{2}, \cdots, \mathbf{p}_{N}$, in the canonical ensemble, the average of a physical quantity $A$ is given by

$$
\langle A\rangle=\frac{\int d \mathbf{p}^{N} d \mathbf{r}^{N} \exp (-\beta E) A\left(\mathbf{p}^{N}, \mathbf{r}^{N}\right)}{\int d \mathbf{p}^{N} d \mathbf{r}^{N} \exp (-\beta E)},
$$

where

$$
\frac{\exp (-\beta E) A}{\int d \mathbf{p}^{N} d \mathbf{r}^{N} \exp (-\beta E)}
$$

is the probability of finding the system in a microstate $\left\{\mathbf{r}^{N}, \mathbf{p}^{N}\right\} \equiv\left\{\mathbf{r}_{1}, \mathbf{r}_{2}, \cdots, \mathbf{r}_{N}\right.$, $\left.\mathbf{p}_{1}, \mathbf{p}_{2}, \cdots, \mathbf{p}_{N}\right\}$ with energy $E$ at temperature $\beta=1 / k_{B} T$. The integration in Eq. (3.1) is over all microstates in phase space.

In statistical mechanics the evolution of a $N$-particle system is conceptualized in terms of its evolution in phase space. The phase space is a hypothetical $6 \mathrm{~N}$-dimensional space where $3 \mathrm{~N}$-dimensions correspond to the positions of configurational space and the remaining $3 \mathrm{~N}$-dimensions correspond to the conjugate momenta of momentum space. Therefore, each point in phase space, $\Gamma$, is a function of $\left\{\mathbf{r}_{1}, \mathbf{r}_{2}, \cdots, \mathbf{r}_{N}, \mathbf{p}_{1}, \mathbf{p}_{2}, \cdots, \mathbf{p}_{N}\right\}$. At equilibrium, the averages of any physical quantities are calculated as ensemble averages using Eq. (3.1).

In the ensemble approach, physical quantities are calculated from the representative sample microstates as described above. The microstates in this case are several mental copies of the system with different microscopic details (different particle positions and velocities) but obeying the same macroscopic conditions such as constant energy, constant temperature, etc. Alternatively, physical prop- 
erties can also be calculated using time averages in place of ensemble averages. At equilibrium, due to dynamic evolution of the particle positions and velocities, the system explores different microscopic states in phase space under the same macroscopic conditions. Then, time averages are calculated by computing averages of desired physical property over the phase space trajectory. This method of calculating quantities is analogous to experimental measurements where properties of interest are calculated as averages over the time period of measurement. In the limit of $t \rightarrow \infty$, time averages should be identical to the ensemble averages. This is known as the ergodic hypothesis and forms the crucial connection between molecular dynamics and statistical mechanics [59]. Note the ergodic hypothesis is valid only in an equilibrium situation [61]. In nonequilibrium studies, the macroscopic state of the system changes in time due to external perturbations and clearly the ergodic hypothesis is not applicable for such studies.

\subsection{Implementation}

Molecular dynamics in many ways resembles real experiments. Just like in experiments, one has to prepare the sample under desired conditions, let the system reach the desired state and then measure the properties of interest. Because of this, a MD simulation is often referred to as a virtual experiment or computer experiment. A typical MD simulation consists of the following steps:

1. Initialization.

2. Evaluation of potentials.

3. Solving the equations of motion under right conditions.

4. Time integration.

5. Obtaining time averages.

We now give a brief overview of all these points.

\subsubsection{Initialization}

Every simulation starts with an initial configuration. In molecular dynamics, the initial configuration is specified by assigning positions and velocities to all the constituent particles. Often in simulations particle positions are chosen such that the initial configuration is close to the system's equilibrium structure. While lattice structures are straightforward intuitive choices as initial configurations for solid systems, what about homogeneous fluids? Fortunately, lattice structures such as the face-centered cubic (fcc) work just as well for liquid systems [63]. With liquids additional attention must be paid in choosing appropri- 
ate lattice constants so that the density is compatible with that of the system. In the case of liquids, particles are placed on a lattice that closely resembles their equilibrium structure. After assigning initial positions and velocities, the system is equilibrated for a short period until it acquires its equilibrium structure. During this equilibration period the initial configuration on the lattice melts and the liquid finds its equilibrium structure. Ideally, the initial configuration should be such that the system under study relaxes to its equilibrium structure as quickly as possible [59]. Introduction of a small random displacement about the initial particles positions on the lattice has been shown to speed up the melting process of the lattice structure and thus helps the system to reach its equilibrium structure faster [64]. The random displacement must be small enough to avoid any possible overlaps between particles.

However, in the case of molecular systems it is also necessary to include information regarding orientation of the molecular constituents [59]. Preparing initial configurations for molecular systems may not be straightforward. Fortunately, lattice structures with appropriate lattice constants work for molecular systems also. Apart from using lattice structures another method for creating initial configurations is by randomly placing molecules in a specific volume to realize the system at a particular density. Due to its very nature, this approach may lead to unphysical overlaps between different entities of the system [59]. However, the system can be relaxed to its equilibrium configuration by the application of a suitable mechanism. We will introduce one such mechanism used in our simulations in the next chapter.

Having assigned positions to all the particles, the next step is to select particle velocities according to the temperature of the system. Often, initial velocities are assigned from the Maxwell-Boltzmann distribution in accordance with the operating temperature [59]. Another method is to select velocities from a uniform random distribution $[59,61]$. Normally, in this case velocities assume the Maxwell-Boltzmann distribution rapidly [59]. We use this latter method for generating initial velocities. After choosing one of the above two methods to generate the initial velocity distribution, then the individual velocities are rescaled to match the desired temperature. For equilibrium studies, it is important to ensure that the total initial vector momentum is zero to avoid any drift of the system [65]. Once the initial configuration of the system is set the next step is to define interactions between various constituents of the system. 


\subsubsection{Potentials}

The nature of a system is contained in the various types of interactions that are present between its constituents. These interactions are implemented by including the potential energy functions into the Hamiltonian of the system. Depending on the system under study and the properties of interest either classical or ab-initio MD methods are employed. In our dissertation work, we only deal with the classical form of molecular dynamics and hence limit our discussion to this class of MD. Before we proceed to discussing potentials used in our work we would like to briefly discuss the paradigm of classical molecular dynamics.

Complete description of intra- and inter-atomic interactions of any physical system demands quantum mechanical treatment. The Hamiltonian representation of a system at atomic level can be written as [58]

$$
\mathscr{H}=\mathscr{K}_{e}+\mathscr{K}_{n}+\mathscr{V}_{e e}+\mathscr{V}_{n n}+\mathscr{V}_{e n}
$$

where $\mathscr{K}$ and $\mathscr{V}$ indicate the kinetic and potential energies, respectively and the subscript ' $e$ ' refers to electrons and ' $n$ ' refers to the nuclei. Using a quantum mechanical picture also means implementation of complex potentials. As a consequence, corresponding simulations will be computationally very intensive and time consuming; limiting their application to small systems. Fortunately, a classical description is found to be adequate for many practical applications [59].

Classical MD operates under the Born-Oppenheimer approximation [66]. According to this approximation, since the mass of nuclei are much heavier than electrons, the electronic motions quickly adjust to any change in the position of nuclei. This allows us to decouple the Hamiltonian into electronic and nuclear parts. Assuming that the electronic motions are much faster than the nuclear motion, electronic motion averages out leaving the Hamiltonian as a function of nuclear variables only [59]. In addition to this approximation, in classical MD, it is also assumed that the interaction between particles can be described by potential energy functions. For the sake of simplicity, from here on, we refer to classical MD simply as molecular dynamics.

The general form of a potential function of a many body system can broken down as [59]

$$
\mathscr{V}=\sum_{i} v_{1}\left(\mathbf{r}_{i}\right)+\sum_{i} \sum_{j>i} v_{2}\left(\mathbf{r}_{i}, \mathbf{r}_{j}\right)+\sum_{i} \sum_{j>i} \sum_{k>j} v_{3}\left(\mathbf{r}_{i}, \mathbf{r}_{j}, \mathbf{r}_{k}\right)+\cdots \cdots
$$


The first term in the above equation is due to the external field. The second term represents pair interactions between particles. The argument of this term usually reduces to the pair-wise distance $r_{i j} \equiv\left|\mathbf{r}_{i}-\mathbf{r}_{j}\right|$, between particles $i$ and $j$. The third term is due to triplet interactions and so on. In general, the potential functions are truncated at the pair term and higher order terms are neglected as they are computationally expensive. However, many body effects can be incorporated via pair potentials by replacing them with some effective potentials. This approach is found to be adequate for many practical situations. The strategies involved in constructing these effective potentials will be discussed later in the Lennard-Jones potential section.

Most of the potentials that are used in simulations to describe the systems are empirical in nature. Surprisingly, empirical potentials are found to be adequate in modelling many particle systems. These potentials are constructed based on experimental results, structural information, etc [67, 68]. For example, in a molecular system the potential energy should a function of bond lengths, bond angles, torsion angles, etc. The potential function of a simple molecular system has the following form

$$
\Phi=\Phi_{\text {bond }}+\Phi_{\text {angle }}+\Phi_{\text {torsional }}+\Phi_{\text {non-bonded }}
$$

where the first term on the right hand side of Eq. (3.5) accounts for any fluctuations in the bond lengths. The term $\Phi_{\text {angle }}$ models the penalty associated with any deviations in the bond angles between different bonded entities of the molecules, from their equilibrium values. The third term is the torsional angle term. Torsional angle is defined as the angle between two intersecting planes where the first plane contains the first three monomers of the quadruplet and the second plane is constructed by joining the last three. Fig. 3.1 shows the schematic diagram of bond- and torsion-angles. The final term in Eq. (3.5) is due to the interaction between non-bonded entities. Note each of the terms in Eq. (3.5) correspond to a term in Eq. (3.4).

Depending on the problem and nature of study, further approximations can be made in addition to neglecting the quantum mechanical effects. Moreover, our limited understanding of various types of interactions hinders us from constructing potentials that can precisely capture all features of the system. In many cases, we are interested in the general behaviour of the system rather than the exact, and application of detailed potentials that encapsulate all features is not necessary in such situations. Therefore, in simulations, there is greater emphasis on using "generic" potentials that capture essential physics while reducing the 


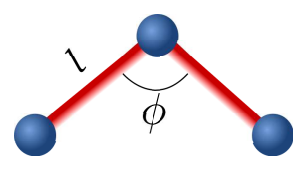

(a)

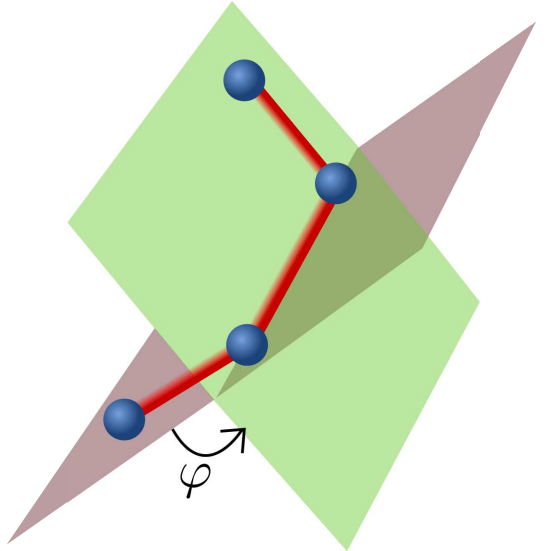

(b)

Figure 3.1: (a) Bond angle $\phi$, (b) Torsion angle $\psi$. The blue spheres represents monomers and the red cylinders represent the connecting bonds.

complexity of the problem and thus being computationally economical. In general, the generic potentials commonly used in simulations describe the underlying phenomena very well. One of the most commonly used generic potential is the Lennard-Jones potential.

\section{The Lennard-Jones Potential}

The Lennard-Jones (LJ) potential is one of the most successful and widely used potentials in MD simulations. Its widespread use is due to its universal appeal in correctly describing the pair-wise interactions between particles interacting via van der Waals forces. The LJ potential consists of two parts, (a) short-range repulsion, and (b) long-range attraction. The short-range repulsion is due to Pauli's principle. When the atoms or molecules come very close to each other in space, their electronic clouds start to overlap causing an abrupt increase in their potential energy which eventually pushes them apart. On the other hand, the longrange attraction term is due to weak dispersion forces originating from the fluctuations in dipole moments of molecules [63]. The $12-6$ form of LJ potential is given by

$$
\Phi_{L J}(r)=4 \varepsilon\left[\left(\frac{\sigma}{r}\right)^{12}-\left(\frac{\sigma}{r}\right)^{6}\right]
$$

where $\varepsilon$ and $\sigma$ correspond to the interaction energy and the size of the particles respectively, and $r$ is the distance between the particles. The parameters $\varepsilon$ and $\sigma$ are system dependent. The $r^{-12}$ term represents the short-range repulsion and the $r^{-6}$ term is due to the weak long-range attraction. The $12-6$ form is the most commonly used form of the LJ potential because of the computational benefits it has to offer [65]. The typical LJ potential as a function of distance, $r$, is shown in 
Fig. 3.2.

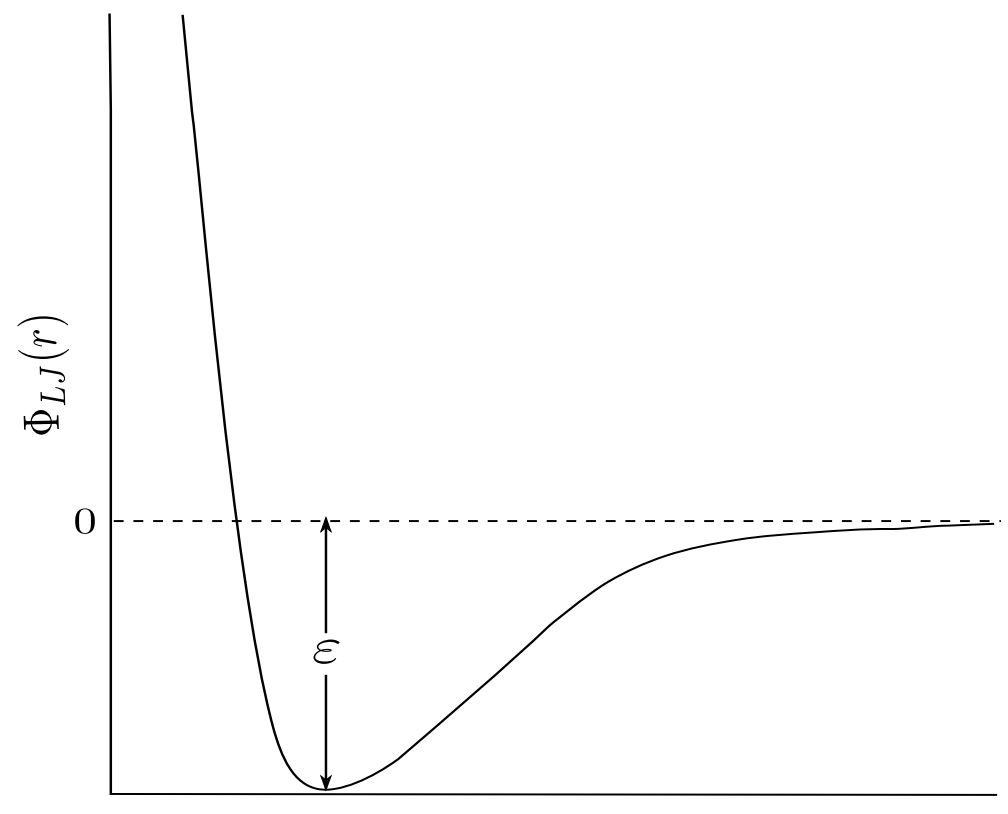

Figure 3.2: The Lennard-Jones potential.

Initial simulations using the LJ potentials were carried out to study rare earth gases such as Ar [59]. The LJ potential successfully models these systems because of their closed electronic shells and since particles interact mainly via dispersion forces. But because of its ability to emulate such interatomic interactions the LJ potential is widely used as a model or test potential for many systems.

As mentioned previously, many body interactions are integrated into simulations via effective potentials. We will explain this concept using the LJ potential as an example. The LJ potential in its original form represents interactions between two individual particles that are separated by a distance $r$ as given by Eq. (3.6). Many body interactions can be incorporated into this equation by fine-tuning the parameters $\varepsilon$ and $\sigma$ to match the experimental results as closely as possible. The LJ potential with these modified parameters no longer represents the interactions between two individual particles but an effective interaction which includes many body effects also [59].

\subsubsection{Coarse Graining}

In this dissertation, we deal with flexible linear polymer molecules. To model these polymers we make use of the concept of coarse graining. The coarse graining methods are at the core of modelling polymers in computer simulations because of the several advantages they offer [15]. Here we present a brief introduction to the concept of coarse graining in the context of polymer modelling. 
Unlike simple fluids, polymer systems exhibit a range of length- and timescales [15]. The origin of these different scales stems from the connectivity (due to bonds) between different constituents of the chains. To give an example, in a long polymer chain, the bond length might be $\sim 1 \AA$, the persistence length $\sim 10 \AA$ and the coil radius $\sim 100 \AA$ [15]. The persistence length is defined as the length over which the chain appears to be rigid. Different time-scales arise from vibrations in the bond lengths, angles and torsional angles. Thus multiscale modelling methods must be employed for studying properties of polymers as they link salient features on different spatial- and (or) temporal-scales [16]. The properties of the polymers are connected to different length- and time-scales and hence depending on the properties of interest, certain degrees of freedom can be excluded. Based on the scale of operation, molecular simulations can be categorized into, ( $a$ ) electronic level (quantum chemistry), (b) atomic level (force field), or (c) monomeric level (mesoscopic models) [19]. Here we will only focus on mesoscopic models which are of most interest to us.

The basic entity of a polymer chain which repeats itself along the chain backbone is called a monomer. The chemical structure of monomers, in general, is very complex at the atomic level. Therefore, the potential energy functions will be very complicated and cumbersome; and hence impractical to implement if we took into account all the degrees of freedom. Such models are not only computationally expensive but also irrelevant for many studies. In mesoscopic models, a monomer is treated as a single effective unit interacting with similar units, while the chemical structure of the monomer is completely ignored [15]. This method of reducing the complexity of a molecular system is known as coarse graining. In some cases, further coarse graining may be desired and this is achieved by grouping together numbers of monomers and treating the resultant as a single monomer.

At first sight, one might feel that coarse graining methods are very crude approximations and will not be able to provide sufficient insight into the system under study. Fortunately, many properties of macromolecules display universal behaviour that is independent of the chemical structure of monomers [51]. Therefore, coarse grained methods can be successfully applied at studying universal properties of macromolecules. For example, the radius of gyration $\left(R_{g}^{2}\right)$ of a flexible chain is proportional to $N^{v}$, where $N$ is the number of monomeric units in the chain and $v$ is the exponent that depends on the solvent conditions. By exploiting this aspect of polymers in simulations, effective potential energy functions are defined to model the interaction between coarse grained monomers. 


\subsubsection{The Finitely Extensible Nonlinear Elastic (FENE) Potential}

This potential is based on the concept of a bead-spring model. In this model, a polymer chain is represented as a string of beads (monomers) connected to each other via spring-like bonds. The bead-spring models are the most efficient and effective for modelling flexible polymer chains [15].

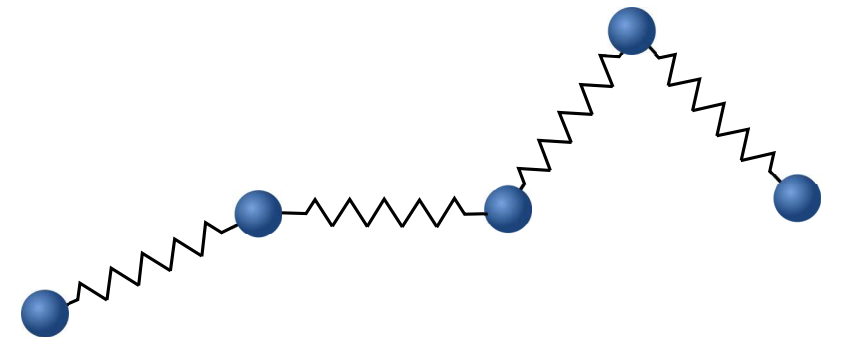

Figure 3.3: The bead-spring model. The blue spheres are the monomers connected by bonds represented by springs (black).

In Fig. 3.3 we show a schematic diagram of the model. The most popular potential based on the bead-spring model is the FENE potential [56, 69]. This potential captures the essential behaviour of polymers thus making it one of the most widely used in computer simulations of polymers [15]. In the FENE model, the monomers are connected to each other via nonlinear springs and it is written down as

$$
\Phi_{\text {FENE }}= \begin{cases}-\frac{1}{2} k r_{0}^{2} \ln \left[1-\left(\frac{r}{r_{0}}\right)^{2}\right] & \text { for } r \leq r_{0} \\ \infty & \text { for } r>r_{0}\end{cases}
$$

where $k, r_{0}$ and $r$ are the spring constant, maximum allowed bond length and the current bond length, respectively. The FENE potential is plotted in Fig. 3.4.

The FENE potential has obvious advantages over the harmonic-spring model [15]. In the harmonic-spring model, the bond extension has Hookean behaviour and does not impose any restrictions on the maximum extension a bond could take. Hence large unphysical bond extensions are not prevented in this model [15]. The FENE model rectifies this shortcoming by imposing an upper limit on the maximum extensibility. For small bond extensions, the FENE potential behaves like a harmonic spring, but once the bonds are stretched to their maximum limit it rises sharply. This prevents unphysical over-stretching of bonds.

However, excluded volume interactions are not included in the FENE model. For this purpose, the LJ potential is employed alongside with the FENE potential to prevent monomers from overlapping. This combination adequately describes the bond interactions for our purposes. 


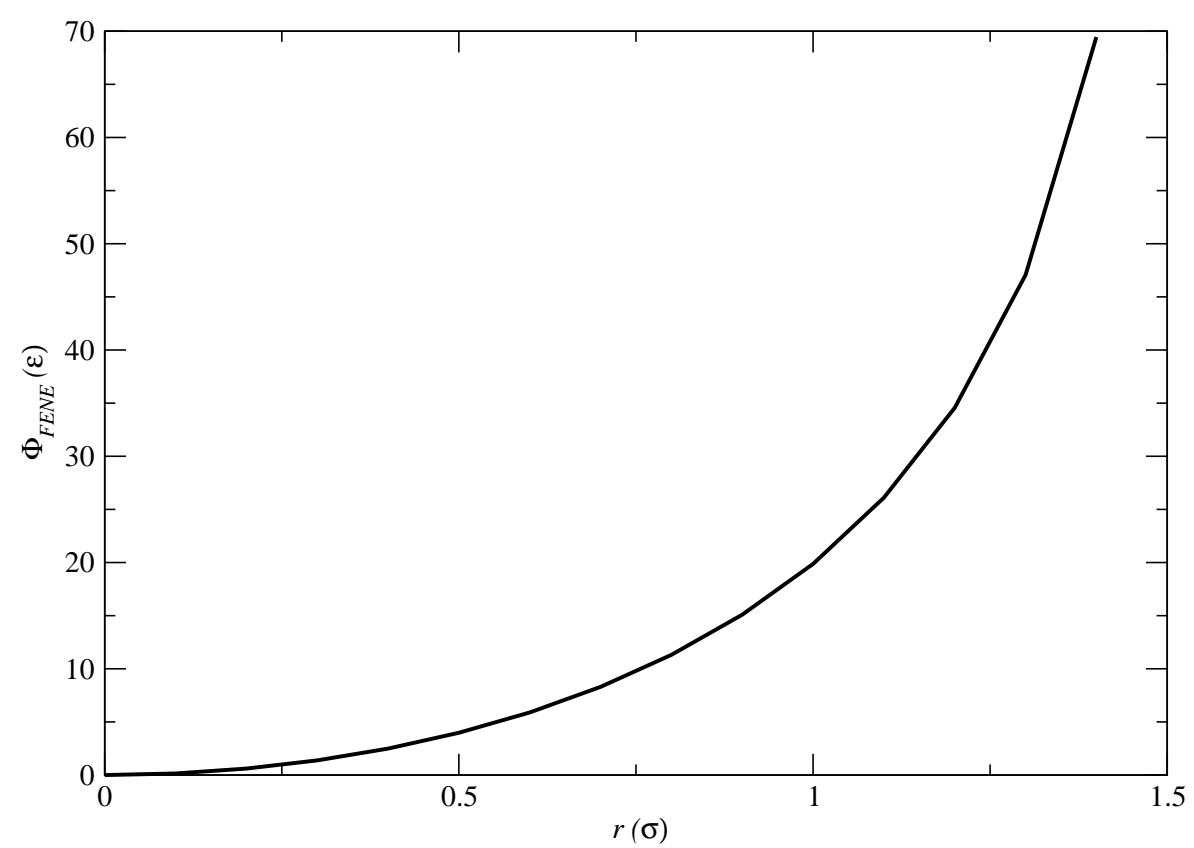

Figure 3.4: The FENE potential as a function of bond extension.

\subsection{Ensembles}

The system under study can be exposed to various constraints depending on the nature of the study. The phase space trajectory explored by the system is subjected to the initial conditions and constraints under which it operates i.e., constant energy (NVE), constant temperature (NVT), isothermal-isobaric (NPT), etc. We will only discuss constant energy (NVE) and constant temperature (NVT) ensembles here which are relevant to our work. For detailed discussion on different ensembles the reader is referred to sources $[59,61]$.

\subsubsection{Microcanonical Ensemble (NVE)}

In this ensemble, the number of particles $(N)$, volume $(V)$ and energy $(E)$ of the system remain constant. Systems that obey these constraints can be thought of as "isolated systems" in that they do not exchange energy with their surroundings. In this ensemble, the phase space evolution takes place due to exchange between kinetic and potential parts of energy while the total energy remains constant. The allowed microstates lie on an energy surface in phase space that satisfies the constant energy criteria. In practice, a small window of energy $E$ to $E+\delta E$ is considered and therefore microstates lie in a hypershell of thickness $\delta E$ in phase space. In this ensemble, the probability of finding the system in any of these possible microstates is equally likely.

In an NVE simulation, the temperature of the system is directly calculated 
from its kinetic energy using the equipartition theorem which states that kinetic energy per degree of freedom is equal to $k_{B} T / 2$. Implementation of this ensemble in MD simulations is quite straightforward. By default, Newton's equation of motion conserves energy and the subsequent phase space trajectories generated by solving these equations sample the microcanonical ensemble.

\subsubsection{Canonical Ensemble (NVT)}

The NVT ensemble is widely used in MD simulations because of its practical relevance. In this ensemble, the number of particles $(N)$, volume $(V)$ and temperature $(T)$ of the system remain constant. In this ensemble the system is assumed to be in contact with a much bigger heat reservoir and it is allowed to exchange heat (energy) with the reservoir. At equilibrium, the system comes to thermal equilibrium with the reservoir. The probability of finding the system in a particular microstate with energy $E_{i}$ is given by the Boltzmann distribution

$$
P_{i}=\frac{e^{-E_{i} / k_{B} T}}{\sum_{j} e^{-E_{j} / k_{B} T}} .
$$

where the summation index $j$ spans over all states.

The temperature of a system is derived from the kinetic energy which in turn is a function of particle velocities. Therefore, by adjusting particle velocities the temperature of the system can be controlled. There are several methods to realize a canonical ensemble or a NVT simulation in molecular dynamics [59, 61]. Simplest of all is the velocity rescaling method based on the equipartition theorem [70]. In this method thermostating is achieved by rescaling the particle velocities to meet the constant temperature condition. Since the kinetic energy is fixed in strict terms, the momentum space cannot be explored properly [71]. This also leads to unrealistic dynamics.

Broadly speaking thermostats can be categorized into two groups depending on the method of implementation, (a) stochastic, or (b) deterministic [71]. Though there are several thermostats that are used to realize constant temperature molecular dynamics we limit our discussion to:

1. the Langevin thermostat and

2. the Nose-Hoover thermostat

as they are relevant for the work presented in this dissertation. 


\section{Langevin Thermostat}

This thermostat is based on stochastic dynamics. The system particles are assumed to be immersed in a fictious solvent. Solute (system) particles experience two types of forces due to their interaction with the solvent particles: a frictional force and a random force. The physical origins of these forces can be understood in the following way. Due to the movement of solute particles in the solvent, the solvent particles exert a frictional drag resisting the motion of solute particles. This is modelled as a frictional force on the particles. During their movement in the solution, solvent particles randomly collide with the solute particles and this effect is modelled as a random force. The frictional and random forces are related to each other via the fluctuation-dissipation theorem [72]. Including the frictional and random force terms into the equations of motion yields

$$
m_{i} \ddot{\mathbf{r}}_{i}(t)=-m_{i} \gamma_{i} \dot{\mathbf{r}}_{i}(t)+\mathbf{F}_{i}+\mathbf{R}_{i}
$$

where $\mathbf{F}_{i}$ is the force experienced by particle $i$ due to its interaction with all other particles. The first term on the right hand side of the above equation represents the frictional drag on particle $i$ with friction coefficient $\gamma_{i}$. The term $\mathbf{R}_{i}$ is the random force that mimics the effect of random kicks due to solvent particles on the solute particle $i$. By adjusting the frictional and random forces while obeying the fluctuation-dissipation theorem, constant temperature simulations can be realized. The random force $\mathbf{R}_{i}$ satisfies the following conditions [71]

- $\mathbf{R}_{i}$ is a Gaussian process with zero mean. This approximation is valid if the mass of the Brownian particle is much larger than that of the solvent particles. This approximation leads to the following probability distribution for $\mathbf{R}_{i}$

$$
\begin{aligned}
W\left(\mathbf{R}_{i}\right) & =\frac{1}{\left(2 \pi\left\langle R_{i}^{2}\right\rangle\right)^{1 / 2}} e^{-R_{i}^{2} / 2\left\langle R_{i}^{2}\right\rangle} \\
\left\langle\mathbf{R}_{i}\right\rangle & =0
\end{aligned}
$$

Here $<>$ denotes the ensemble average. Since on average a particle experiences zero force, the random force term does not have any effect on the sampled phase space. As a consequence, the generated trajectory represents a canonical ensemble.

- It is also essential that $\mathbf{R}_{i}$ has no correlation in time and space. This condition is fulfilled by assuming that the correlation time between any two random collision events is infinitesimally small. The above approximation results in the 
following autocorrelation relationship

$$
\left\langle\mathbf{R}_{i}(t) \mathbf{R}_{j}\left(t^{\prime}\right)\right\rangle=2 m_{i} k_{B} T_{r e f} \delta_{i j} \delta\left(t-t^{\prime}\right) .
$$

In the above equation $T_{r e f}$ is the reference or desired temperature. The Kronecker delta function $\delta_{i j}$ models the spatial correlation and $\delta\left(t-t^{\prime}\right)$ is the Diracdelta function to model time correlation, between particles $i$ and $j$ [73].

Because of its stochastic nature due to random collisions, the Langevin thermostat allows us to use bigger timesteps. This feature is very useful, especially in the study of polymer systems where the relaxation times can be very long [74]. Another advantage of this thermostat is that it permits thermostating individual degrees of freedom separately [73]. This feature is specifically useful in flow simulations where it is desirable to only thermostat chosen degrees of freedom.

\section{Nosé-Hoover Thermostat}

This thermostat falls under the category of deterministic thermostats. In this thermostat, the interaction between the system and reservoir is modelled by introducing an additional degree of freedom [75]. This additional degree of freedom is represented by introducing an extra variable $s$. This variable controls the heat flow in and out of the system. Since an additional degree of freedom is introduced, this method is often referred as the extended system method. Along with the system particle positions and momenta, equations of motion are also solved for this extra degree of freedom $s$. Fluctuations in the variable $s$ determine the direction of heat flow [75]. The artificial variable $s$ relates the real system timestep $(\delta t)$ to the extended system timestep $\left(\delta t^{\prime}\right)$ by the following relation

$$
\delta t^{\prime}=s \delta t
$$

From the above equation it can be inferred that the time-scale in the extended system appears to be stretched by a factor of $s$. As a result of this scaling even though the particle coordinates are the same in both the real and extended systems, velocities differ. The real and extended system velocities are related via

$$
\mathbf{v}_{i}=s \dot{\mathbf{r}}_{i}
$$

where $\mathbf{v}_{i}$ is the velocity of particle $i$ in the real system and $\dot{\mathbf{r}}_{i}$ is the velocity in the extended system. 
The Lagrangian for the extended system is

$$
\mathscr{L}=\frac{1}{2} \sum_{i=1}^{N} m_{i} s^{2} \dot{\mathbf{r}}_{i}^{2}-\Phi(\mathbf{r})+\frac{1}{2} Q \dot{s}^{2}-g k_{B} T_{r e f} \ln s .
$$

The first two terms in the above equation represent the kinetic and potential energy contributions due to the real system. The third is the kinetic energy due to the additional variable $s$ with an effective mass $Q$ and velocity $\dot{s}$. The last term is the potential energy due to $s$. Here $g$ is the number of degrees of freedom and $T_{\text {ref }}$ is the temperature of the extended system (and desired temperature for the real system). The form of the potential energy for the extended system is chosen so that a canonical ensemble is realized [75]. The equations of motion are derived from the Lagrangian (Eq. (3.15)) as

$$
\begin{aligned}
\ddot{\mathbf{r}} & =\mathbf{F} / m s^{2}-2 \dot{s} \dot{\mathbf{r}} / s \\
Q \ddot{s} & =\sum_{i=1}^{N} m_{i} s \dot{s}_{i}^{2}-g k_{B} T_{r e f} / s,
\end{aligned}
$$

and the corresponding conjugate momenta

$$
\begin{aligned}
& \mathbf{p}_{i}=m_{i} s^{2} \dot{\mathbf{r}}_{i} \\
& p_{s}=Q \dot{s} .
\end{aligned}
$$

The resulting Hamiltonian of the extended system

$$
\mathscr{H}=\sum_{i=1}^{N} \frac{p_{i}^{2}}{2 m_{i} s^{2}}+\Phi(\mathbf{r})+\frac{p_{s}^{2}}{2 Q}+g k_{B} T_{r e f} \ln s
$$

is a conserved quantity and this property can be used as a test during the simulation run. It can be shown that the average of a quantity in the extended system samples a canonical ensemble [75]. The trajectories produced using this thermostat are smooth, deterministic and time-reversible [71].

However, there is an inherent problem with Nosé's formulism. The timestep depends on the scaling factor $s$ and fluctuations in $s$ lead to unequal time intervals during the course of the simulation which is not intuitive and makes this method unsuitable for studying dynamic properties [76]. Hoover [76] proposed a modified version of Nosé's formulism that addresses this issue. By eliminating the scaling factor $s$ from the equations of motion, Hoover recovered the following 
set of equations:

$$
\begin{aligned}
\dot{\mathbf{r}} & =\mathbf{p} / m \\
\dot{\mathbf{p}} & =\mathbf{F}(\dot{\mathbf{q}})-\zeta \mathbf{p} .
\end{aligned}
$$

Here $\zeta$ is the friction coefficient given by

$$
\dot{\zeta}=\left(\sum_{i=1}^{N} p_{i}^{2} / m_{i}-g k_{B} T_{r e f}\right) / Q
$$

The time evolution of $s$ can be obtained by solving the second order differential equation given by Eq. (3.17). The solution of $s$ can be oscillatory in behaviour and as a result heat exchange between the system and the reservoir may take place in a periodic fashion which is not desirable [77]. The heat flow can be controlled by adjusting the thermal inertia $Q$. If the value of $Q$ is too high then the energy flow takes place at a slow pace. In the limit $Q \rightarrow \infty$, the system recovers the conventional microcanonical ensemble. On the other hand, if $Q$ is too small it leads to weak, damped oscillations in energy which in turn lead to poor equilibration [59].

\subsubsection{Application to Nonequilibrium Molecular Dynamics}

The discussion of the thermostats above is from the perspective of equilibrium statistical mechanics. All the simulations reported in this dissertation are of nonequilibrium nature. In nonequilibrium mechanics, the system is moved from one equilibrium state to another by the application of an external force and during this process additional heat is produced. In many practical situations it is desirable to remove this excess heat and thermostats can be used for this job [78]. Note, caution must be paid while using thermostats in non-equilibrium situations to avoid any interference that may take place due to thermostating. For example, in Poiseuille flow simulations, aimed at computing longitudinal velocity profiles, it is desirable to be avoid thermostating the fluid in the flow direction, as it will alter the dynamics in that direction.

\subsection{Time integration}

Once all interactions between constituents of the system are specified the next step is to use them to solve Newton's equation of motion. The equation of motion 
of any particle $i$ of the system is

$$
m_{i} \ddot{\mathbf{r}}_{i}=-\nabla \Phi_{i}
$$

where $m_{i}$ is the mass of particle $i$ and $\Phi_{i}$ is sum of all potentials acting on it. Simply solving the above equations of motion results in a microcanonical ensemble. However, imposition of additional constraints such as the one discussed in the previous section alter these equations. For example, the NVT simulation with the Langevin thermostat modifies the equations of motion as in Eq. (3.9).

The dynamical evolution of the system is obtained by solving equations of motion in time. Given the positions and velocities of all the particles at time $t=0$, the system evolution can be found out at any future time $t$. Thus the MD method is deterministic in nature. Though deterministic in principle, the trajectory explored by the system in phase space can be different from that of the actual trajectory because of approximations made at the algorithmic level to improve the efficiency and precision errors that occur at the machine level. However, most often MD simulations are used for calculating thermodynamic/time averages, in which case the errors can be reduced [61]. Thus it is not always necessary to solve the equations of motion exactly provided the time average of the approximate trajectories is close enough to that of the exact trajectories.

Numerically, the time evolution is achieved by discretizing the equations of motion, which are second order differential equations, in time. Choosing a good algorithm for time integration is important. These time integration schemes, because of finite differencing in time, invariably depend on the choice of timestep used. Generally, time evolution is achieved by doing a Taylor series expansion of particle positions and velocities by a small increment in time from $t$ to $t+\delta t$. In practice, the Taylor series expansion is terminated after a certain number of terms and this leads to truncation errors. Consequently, the order of accuracy of the algorithm inherently depends on the timestep size $\delta t$. Other types of error that occur in MD simulations are due to precision errors. Usually, the truncation errors are the ones that need close attention [59].

There are several time integration schemes that are used in MD simulations $[59,61]$. We restrict our discussion to two of the most popular

1. Predictor-Corrector Method.

2. Verlet Integration. 


\subsubsection{Predictor-Corrector Method}

In this method $[79,59,80]$, particle positions, velocities, accelerations and time derivatives of acceleration are predicted by expanding them in the vicinity of their current time $t$. Thus subsequently obtained values are referred to as predicted values.

$$
\begin{aligned}
\mathbf{r}^{p}(t+\delta t) & =\mathbf{r}(t)+\mathbf{v}(t) \delta t+(1 / 2) \mathbf{a}(t) \delta t^{2}+(1 / 6) \mathbf{b}(t) \delta t^{3}+\cdots \\
\mathbf{v}^{p}(t+\delta t) & =\mathbf{v}(t)+\mathbf{a}(t) \delta t+(1 / 2) \mathbf{b} \delta t^{2}+\cdots \\
\mathbf{a}^{p}(t+\delta t) & =\mathbf{a}(t)+\mathbf{b}(t) \delta t+\cdots \\
\mathbf{b}^{p}(t+\delta t) & =\mathbf{b}(t)+\cdots
\end{aligned}
$$

In the above equations, the superscript ' $p$ ' denotes that they are predicted values. The above set of equations do not yield proper time evolution of the system as they do not depend on the force field calculations. The corrector step rectifies this inadequacy.

In the corrector step, the accelerations $\mathbf{a}^{c}(t+\delta t)$ are obtained by substituting for the positions $\mathbf{r}^{p}(t+\delta t)$ into the equations of motion and solving for the accelerations. These corrected accelerations are then used to estimate the error in accelerations by subtracting $\mathbf{a}^{c}(t+\delta t)$ from $\mathbf{a}^{p}(t+\delta t)$,

$$
\Delta \mathbf{a}(t+\delta t)=\mathbf{a}^{c}(t+\delta t)-\mathbf{a}^{p}(t+\delta t) .
$$

The error estimate is then used to find the corrected values

$$
\begin{aligned}
\mathbf{r}^{c}(t) & =\mathbf{r}^{p}(t)+c_{0} \Delta \mathbf{a}(t+\delta t) \\
\mathbf{v}^{c}(t) & =\mathbf{v}^{p}(t)+c_{1} \Delta \mathbf{a}(t+\delta t) \\
\mathbf{a}^{c}(t) & =\mathbf{a}^{p}(t)+c_{2} \Delta \mathbf{a}(t+\delta t) \\
\mathbf{b}^{c}(t) & =\mathbf{b}^{p}(t)+c_{3} \Delta \mathbf{a}(t+\delta t)
\end{aligned}
$$

where $c_{0}, c_{1}, c_{2}, c_{3}$ are constants. It may be necessary to carry out several iterations at the corrector level to acquire the required degree of convergence.

This is a disadvantage since often the most time consuming part of a MD algorithm is the force field calculations. Several iterations at the corrector step means several force field evaluations. Thus multiple force field calculations at the corrector step severely hampers the progress of the simulation. This is the major drawback of the predictor-corrector methods. Another disadvantage with these algorithms is that they are not time reversible. 


\subsubsection{Verlet Algorithm}

By far the most widely used method for performing time integration in MD simulations, was proposed by Verlet [81]. Let $\mathbf{r}(t), \mathbf{v}(t)$ and $\mathbf{a}(t)$ be the positions, velocities and accelerations of the particles at time $t$. By doing a Taylor series expansion for the positions $\mathbf{r}$ in the vicinity of $t$, one arrives at:

$$
\begin{aligned}
& \mathbf{r}(t+\delta t)=\mathbf{r}(t)+\mathbf{v}(t) \delta t+(1 / 2) \mathbf{a}(t) \delta t^{2}+(1 / 6) \mathbf{b}(t) \delta t^{3}+O\left(t^{4}\right)+\cdots \\
& \mathbf{r}(t-\delta t)=\mathbf{r}(t)-\mathbf{v}(t) \delta t+(1 / 2) \mathbf{a}(t) \delta t^{2}-(1 / 6) \mathbf{b}(t) \delta t^{3}+O\left(t^{4}\right)+\cdots
\end{aligned}
$$

Here $\delta t$ is the timestep size. Adding the above two equations yields

$$
\mathbf{r}(t+\delta t)=2 \mathbf{r}(t)-\mathbf{r}(t-\delta t)+\mathbf{a}(t) \delta t^{2}+O\left(\delta t^{4}\right)
$$

By substituting for the positions at $t$ and $t-\delta t$ and accelerations at $t$ into Eq. (3.30), we obtain positions at the next timestep $t+\delta t$. The accelerations $\mathbf{a}(t)$ are computed from Eq. (3.24). This algorithm is accurate to order $\delta t^{4}$. This gives us the freedom of choosing relatively large timesteps.

Using Eqs. (3.28) and (3.29), we can calculate the velocities at time $t$. Though velocities are not part of the time-stepping process, they do play an important role in evaluating quantities like the kinetic energy which can be used to test for energy conservation, for example. By subtracting Eq. (3.28) from Eq. (3.29), we have

$$
\mathbf{v}(t)=\frac{\mathbf{r}(t+\delta t)-\mathbf{r}(t-\delta t)}{2 \delta t}
$$

This equation is correct to an order of $\delta t^{2}$. Thus, choosing large timesteps can lead to deviations in the total energy at long times [82].

The Verlet algorithm has several advantages over the predictor-corrector methods. The time advancement is purely a function of particle positions, this reduces the amount of memory required. From Eq. (3.31) it can also be inferred that time reversal is possible in this method. Unlike in the predictor-corrector methods advancement of positions occurs in one step in the Verlet method. This considerably reduces the computational effort required. The Verlet algorithm also shows good energy conservation even for long timesteps and also conserves linear momentum for conserved forces [59].

However, the Verlet algorithm does not work particularly well in computing velocities because it is only accurate up to $O\left(\delta t^{2}\right)$. Consequently large timesteps can cause fluctuations in velocities, kinetic energy and total energy at short time- 
scales. There have been several algorithms proposed to rectify this problem and the most well-known being the velocity Verlet algorithm [83]. In the Verlet algorithm, positions and velocities are evaluated by advancing in half timesteps. These quantities are then used in computing the values of the positions and velocities of the particles at future time $t+\delta t$. After some simple algebra, the final form of time integration equations are

$$
\begin{aligned}
\mathbf{r}(t+\delta t) & =\mathbf{r}(t)+\mathbf{v}(t) \delta t+(1 / 2) \mathbf{a}(t) \delta t^{2} \\
\mathbf{v}(t+\delta t) & =\mathbf{v}(t)+(1 / 2)\{\mathbf{a}(t)+\mathbf{a}(t+\delta t)\}
\end{aligned}
$$

This algorithm is also a two step process like the predictor-corrector methods but differs in that it involves calculation of variables $\mathbf{r}, \mathbf{v}$ and $\mathbf{a}$ at times $t$ and $t+\delta t$. But this additional computation results in a much more stable numerical solution. Ultimately, this leads to much better phase space trajectories, good energy conservation and thus makes it the most widely used time integration method in MD [59].

\subsection{Tools}

Practical implementation of MD simulations involves application of some additional tools to improve the efficiency of the algorithm. In this section we discuss some of the key tools that are an integral part of a typical MD simulation.

\subsubsection{Periodic Boundary Conditions}

Molecular dynamics can be applied to study the behaviour of both small and large systems. Properties of systems with a few hundreds to thousands of particles, like nanoclusters, nanoparticles etc., can be studied with reasonable accuracy using the MD method discussed so far. However, as the system size increases it becomes computationally very expensive to conduct MD simulations thus computing bulk properties becomes difficult. This problem can be overcome by the application of periodic boundary conditions [59].

A system with periodic boundary conditions is replicated infinitely in all directions. The central cell defines the actual simulation domain and all other surrounding (ghost) cells contain images of the particles that are within the central cell. The coordinates for these images are obtained by adding a simple translational vector to the original coordinates. Images of the particles interact in the same manner as the original particles they represent. If a particle $i$ moves from the central cell to one of its neighbouring cells, all its images move in the same 
fashion and one of the images of the particle enters into the central box from the opposite side. Thus the density of the system is preserved at all times. Therefore, the system is infinitely big in size to calculate bulk properties while we only solve equations of motion for the particles that are present in the central cell.

The periodic boundary conditions must be applied with some precautions. All edge lengths of the simulation domain must be larger than any correlations that may be present in the system. If any of the correlation lengths present in the system are longer than the edges, they will be suppressed because of the periodic boundary conditions. Additional information on various circumstances in which the periodic boundary conditions fail can be found in [59].

\subsubsection{Limiting the Interaction Range}

Most potential energy functions have an infinite interaction range, but in most cases the potential energy becomes diminishingly small at large distances. Therefore, one can impose a finite radius for interactions thus giving a more efficient way of implementing these potentials in simulations. The value of the cut-off radius must be large enough to encompass all important contributions. But the truncation at a finite distance causes abrupt jumps in the force and energy. This in turn effects the conservation of energy [65] which is a crucial test in many simulations. This problem can be overcome by shifting the potential so that the potential and force go to zero smoothly at the cut-off radius. The shifted potential energy has the following form:

$$
\Phi(r)= \begin{cases}\Phi(r)-\Phi\left(r_{c u t}\right)-\left[r-r_{c u t}\right]\left\langle\frac{d \Phi}{d r}\right\rangle_{r_{c u t}} & \text { for } r \leq r_{c u t} \\ 0 & \text { for } r>r_{\text {cut }} .\end{cases}
$$

When applied in combination with periodic boundary conditions, one must ensure that the range of interaction $\left(r_{c u t}\right)$ is always less than half of the smallest edge of the system. This prohibits interaction of particles with their own images.

\subsubsection{Neighbour-list Building}

The most time consuming part of a typical MD simulation are the force field calculations. Most simulations use pair potentials to model the interactions and this involves finding neighbours that are within a certain cut-off radius from the central particle. Assuming that the potential is pair-wise additive, for a system of $N$ particles, $\mathrm{O}\left(\mathrm{N}^{2}\right)$ pair distances must be computed. This number grows inordinately large with a modest increase in $N$ and becomes impossible to deal with. 
Moreover, out of this only a small fraction have significant contributions in the calculations.

Hence we make a simplification. Each particle interacts only with other particles which are within the cut-off distance $\left(r_{c u t}\right)$ from its centre. Special attention must be paid to ensure any particle $i$ only interacts with another distinct particle $j$ of the system or an image of $j$ from one of the neighbouring ghost cells, $j^{\prime}$ but not with both. This is called the minimum image convention.

In liquids, the local environment around particles does not change significantly over a small number of timesteps. That is, neighbouring particles around any particle change only over sufficiently large periods of time. This aspect can be utilized to decrease the amount of computation devoted to calculating the $O\left(N^{2}\right)$ pair distances at every timestep. Verlet [81] proposed a neighbour-list building technique based on the above observations. In this method, in addition to the interaction sphere (in 3D) of radius $r_{c u t}$, an additional skin, $r_{\text {skin }}$, is considered in the neighbour-list construction. Each particle then consists of a neighbour-list that includes particles within the radius $r_{c u t}+r_{\text {skin }}$ surrounding it. The skin depth $r_{\text {skin }}$ accounts for any particles that may enter the interaction region at a later time and hence the force field evaluations will not miss any particles that may contribute to it. Therefore, it is quite important to choose the correct $r_{\text {skin }}$ value to ensure that no interactions are missed out. Once the neighbour-list for all particles is constructed, for the next few timesteps, force field evaluations just use the neighbourlist of the representative particle to calculate pair distances. This list has to be rebuilt every certain number of timesteps to account for any local changes that may occur and to yield proper dynamics. There is a fundamental connection between the frequency of the neighbour-list build and the skin distance, $r_{\text {skin }}$. If $r_{\text {skin }}$ is too small, the skin region is going to be narrow. Thus there is a greater probability for the constituents within the $r_{c u t}+r_{\text {skin }}$ region to change considerably over a short period of time and therefore demanding more frequent neighbour-list building.

\subsubsection{Parallelization}

So far we have only discussed MD simulations that can be run on single processors. This form of computing is known as serial computing. Even with the most powerful processors it is difficult to study large systems because of the computational times and memory involved grow very large [84]. Also, in simulations relating to nonequilibrium studies the macroscopic state of the system changes slowly requiring long periods of time to attain steady state [84]. The dynamics of systems such as proteins, biomembranes and polymers take place over long 
periods of time. In all the above mentioned instances serial computing can be computationally very demanding. These issues can be addressed using parallel computing [85].

With the appearance of modern supercomputers such studies are feasible today. Supercomputers are multiprocessor systems where the number of processors can be tens to hundreds of thousand. These machines are designed to tackle large scale tasks. The task at hand is divided into a number of sub-tasks and each of them are assigned to one of these processors. Different processors handling different sub-tasks exchange information needed to perform the operation as a whole. This method of distributing a single task to multiple processors is known as parallelization.

Molecular dynamics simulations are inherently parallel in nature [84], making MD problems ideal for high performance supercomputers. With the ever increasing power of supercomputers some of the computer experiments that were not possible previously have been revisited. As an example, a recent MD study of cubic copper of $3.2 \times 10^{11}$ atoms has been reported [86]. Such a study would not have been possible without parallelization techniques. In MD, parallelization can be accomplished in one of the three ways, $(a)$ atom decomposition, $(b)$ force decomposition, and (c) domain decomposition [84]. All our MD simulations were carried out using LAMMPS [87] package which uses the domain decomposition method for parallelization, hence we restrict our discussion to this method only.

In the domain decomposition, the simulation domain is divided into a number of sub-domains each of which is assigned to a processor. Each processor contains all information regarding the particles of the sub-domain it represents. The force field calculations are performed simultaneously on all processors. Each processor carries out these calculations on the particles it contains. This method of decomposition works particularly well for short-range potentials. Since most of the particles that are needed to perform force-field calculations are present in the same sub-domain i.e., same processor, little communication is required between processors. However, a small number of particles from neighbouring sub-domains (processors) surrounding each sub-domain can potentially contribute to the force field calculations. If a particle moves from one sub-domain to another in the next timestep, its corresponding information is also passed from its previous processor to the current processor. Ideally, we would like the supercomputer to devote most of its productive time to carry out the calculations while minimizing the communication between the processors. To improve the efficiency of calculations performed for particles that lie close to the boundary of the sub-domains, a small 
strip surrounding the sub-domain is considered (see Fig. 3.5). The information of all ghost particles belonging to this strip are stored alongside the constituent particle information on every sub-domain. Now, since each processor also has information on potential neighbours that reside on neighbouring sub-domains (processors), which can contribute towards force-field calculations performed on the processor, additional interprocessor communication is avoided. The interprocessor information exchange is achieved using the message passing interface (MPI) [88].

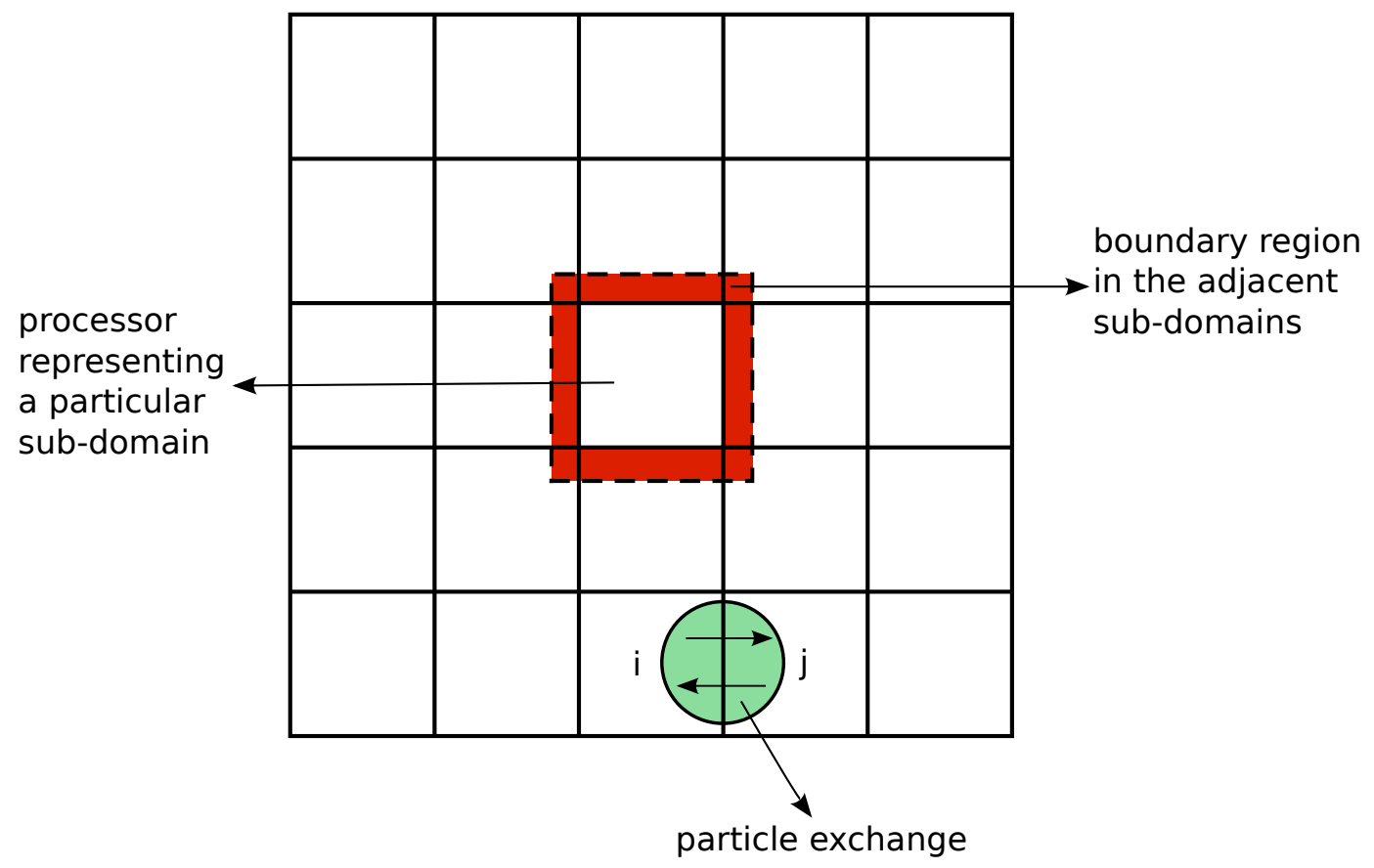

Figure 3.5: Domain decomposition method of parallelization. Each cell represents a sub-domain assigned to a single processor. The red strip indicates the region around the sub-domain belonging to neighbouring sub-domains. Particle information within the strip is made available to the sub-domain to improve the efficiency the algorithm. The green circle shows the particle exchange mechanism between processors. 


\section{Chapter 4}

\section{Flow over Patterned Surfaces}

The study of fluid flow at micro- and nanoscales is fascinating for a variety of reasons. From a fundamental point of view the question that arises is, how does the fluid respond when confined to such narrow dimensions? However, it is the potential applications of this science that attracts the bulk of the research in this area. One of the major goals of microfluidics is to shrink a whole laboratory which is capable of performing chemical and biological analysis onto a chip [20]. Miniaturization of this sort is believed to revolutionize the way chemical and biological processes are performed in a similar way to how integrated circuits transformed the world of computing [21]. Among several advantages of this technology include the need for small volumes of reagents, high sensitivity, high throughput via automation, robustness and portability, to name a few [89, 24, 22]. Applications of this technology would extend to many areas [20, 21, 90, 91].

Flows in micro- and nanofluidic devices are predominantly laminar in nature. Though laminar flows are desirable in some situations [23], they pose severe limitations in many microfluidic applications that require rapid mixing. Due to the laminar nature of the flows, mixing takes place only by virtue of diffusion in these devices. Mixing by such means usually requires long length- and timescales, which makes these devices unsuitable for many practical applications [21, 23]. Furthermore, diffusion driven mixing can be even slower for macromolecular systems, on the order of tens of minutes in some cases [24]. One of the primary goals of microfluidic devices is to be able to perform the biochemical analysis such as drug delivery, cell activation, protein folding, etc. and these processes require fast mixing $[89,22]$. Thus, there is a need to investigate better mixing mechanisms for macromolecular fluids.

Stroock et al. [92] showed that by inducing transverse flows, using patterned 
groove surfaces, mixing rates can be improved. Transverse flows increase the path traversed by the fluid streamlines, for the same channel length, compared to pure laminar flows, thus reducing the required channel lengths for mixing. It has been shown that such transverse flows can be induced using patternedslip surfaces. Hendy et al. [25] have studied the flow of Newtonian fluid over patterned-slip walls, periodic wetting (small slip) and non-wetting (large slip) regions, analytically and compared the results with MD simulations. They concluded that flows over such patterned-slip surfaces enhance mixing significantly. Ou et al. [26] exploited this effect to create a microscale device based on hydrophobic ridges that was found to enhance mixing of an aqueous dye. Pereira [1] has carried out a perturbation analysis of a continuum theory for a viscoelastic fluid with patterned-slip boundary conditions along the lines of Hendy's [25] work. From this study he found that mixing is enhanced for shear-thickening fluids but suppressed for shear-thinning fluids, when compared to Newtonian fluids. Furthermore, by applying a time dependent pressure it has been shown to produce an enhancement of the transverse flows!

Here we study the mixing behaviour of polymer flows over patterned-slip surfaces using MD and compare the results with the continuum theory predictions of Pereira [1]. This chapter is organized as follows. In section 1 we discuss various mixing methods that are used in microfluidic applications. In section 2 we discuss some of important properties of micro- and nanoflows that are relevant to our work and in section 3 we introduce the concept of slip length. In section 4 the simulation setup and initial configuration generation are discussed. In section 5 we carry out Couette flow simulations to determine the nature of the fluid. In section 6 the slip length dependence on the wall-fluid interaction is presented. Finally, in sections 7 and 8 we study the mixing behaviour in a flow over patterned-slip surfaces under different body force conditions. The main findings from this work are summarized in section 9.

\subsection{Mixing Methods}

To achieve rapid mixing, the aim will be to maximize the interfacial area between different streams in as short a time as possible. Clearly, diffusion driven mixing alone cannot meet this demand and alternative mechanisms must be developed to promote transverse flows to speed up mixing. Basically, there are two types of micromixers that are usually employed for mixing in microfluidic devices:

- Passive Mixers: These micromixers promote mixing via molecular diffusion or 
chaotic advection [89], using the topology of the device. These mixers do not consist of any moving parts nor use any external sources of energy for mixing purposes [93]. The advantage of passive micromixers is that they do not wear out easily as they do not have of any moving parts. The drawback of these devices is that they cannot be controlled externally, and hence are inflexible.

- Active Mixers: These micromixers can be classified into two groups depending on whether or not they contain any moving parts. Micromixers that use microstirrer bars, piezoelectric membranes, etc. [93], use moving components, fall under the first category. The second type of active micromixers use perturbations in external fields such as pressure, temperature, electrohydrodynamics, etc. [89]. Active micromixers offer a great deal of flexibility in comparison to passive mixers since they can be controlled externally. However, it is difficult to integrate these mixers into microfluidic devices. Moreover, due to friction generated from moving parts they wear out over time.

A detailed discussion on the various types of passive and active micromixers is presented in the review article [89]. We study a hybrid micromixer that has features of both passive and active micromixers, to enhance the mixing in polymeric fluids. Before venturing into the details of the method employed for this purpose, we think it is appropriate to provide some insight into the underlying physics behind this method.

\subsection{Micro- and Nanoscale Flows}

The area of microfluidics deals with the study of fluid flow and its manipulation on the length scales of micrometre to millimetre [23]. At these scales, fluids act quite differently to their macroscale behaviour. When scaling down from the macro- to microscale, certain phenomena which have negligible effect at the macroscale become more important [21], changing the fluid behaviour at the microscale. Therefore, in order to develop microfluidic devices it is essential to understand the various phenomena that are important at these length scales. Here we introduce some of these aspects which are relevant to our work.

\subsubsection{Laminar Flow}

Mass transport in microdevices is viscous dominated and inertial effects are negligibly small [21]. Typically, in fluid dynamics the flow regime is determined as laminar or turbulent based on the Reynolds number Re. The Reynolds number 
is defined by

$$
\operatorname{Re}=\frac{\rho v L}{\mu},
$$

where $\rho$ is the fluid density, $v$ is the characteristic fluid velocity, $L$ is the characteristic length scale, and $\mu$ is the fluid viscosity. Most microflows have low Re, which means that the flow is basically laminar. As a consequence, streamlines do not cross each other which is a drawback for mixing.

\subsubsection{Surface Area to Volume Ratio}

At the macroscale, the surface area to volume (SAV) ratios are small enough for surface effects not to influence the flow in a major way. But as we scale down, the SAV ratio increases, thus surface effects become ever more prominent. This quantity becomes very large at microscales and the surface forces start to dominate the body forces $[21,91,23]$. This property can be utilized to advantage to overcome some of the limitations posed for fluid flow at small scales.

\subsection{Concept of Slip Length}

The standard text book assumption for fluid flow at the fluid-solid interface is the validity of the no-slip boundary condition [94]. According to this condition the velocity of the fluid at the wall-fluid interface is equal to the velocity of the wall itself, while this condition is found to be adequate for describing macroscale flows, its validity at small length scales has always been debated [94, 95, 96]. In recent years, experimental and simulation studies at the microscale have reported violation of the no-slip boundary condition i.e., they reported finite slip of fluids at the wall-fluid boundary $[97,98,99,100,101,102]$.

The phenomenon of slip is quantified in terms of slip length, which is defined as the distance in a direction perpendicular and into the wall where the linear extrapolation of the longitudinal component of the fluid velocity at the fluid-wall interface becomes zero. The concept of slip length is illustrated in Fig. 4.1.

The property of slip can be used to address some of the shortcomings of micro- and nanoflows. One of the issues with micro- and nanoflows is that at these length scales the resistance to the flow is quite high. It has been demonstrated that the slip phenomenon can be effectively used to tackle this problem $[99,103]$. Thus there have been studies exploring various conditions under which large slip lengths can be actualized. Some studies have reported microscale slip lengths $[97,98,104]$. The slip length has been found to depend on several factors such as surface roughness [103, 105], wettability [105, 106], shear rate dependence 


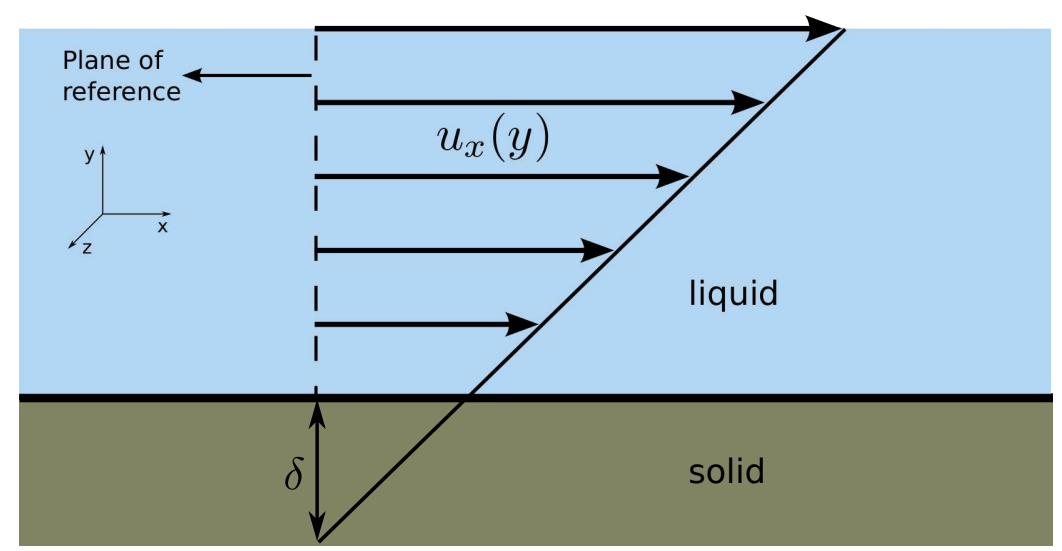

Figure 4.1: The longitudinal velocity profile $u_{x}(y)$ in a Couette flow where the upper wall of the channel is moving in the $x$ direction. The linear extrapolation of $u_{x}(y)$ at the liquid-solid boundary intersects the plane of reference $\delta$ distance into the wall. Here $\delta$ is called the slip length of the flow.

$[100,107,108]$, etc.

\subsection{Molecular Dynamics Simulations}

\section{Simulation Setup}

A coarse grained polymer melt was created by modelling polymer molecules as bead-spring chains with $N=20$ monomers each. The polymer melt was confined between two atomistically flat walls in the $x z$ plane with periodic boundary conditions in $x$ and $z$ directions. The direction of flow was along the $x$ axis. Each wall contained three atomic layer deep fcc (100) atoms. The wall atoms were fixed in space throughout all the simulations reported in this chapter, to reduce the computational effort. Though fixing the wall atoms is unrealistic, it should not have any major implications on results as all simulations were performed under the same conditions. The schematic representation of the simulation setup is shown in Fig. 4.2. Throughout this thesis all the physical quantities are expressed in reduced Lennard-Jones units.

The interaction between the wall atoms and monomers was modelled using the shifted LJ potential (combining Eqs. (3.6) and (3.34)) with the cut-off radius $r_{\text {cut }}=2.5 \sigma$. Density of the walls was chosen to be $0.7 \sigma^{-3}$. The interaction between bonded monomers was modelled using the FENE potential with $r_{0}=1.5 \sigma$ and $k=30 \varepsilon \sigma^{-2}$. This set of parameters prevents unphysical events such as bondbreaking and chain crossing [109]. In addition to the FENE potential, the excluded volume interactions between bonded monomers was included with the help of the shifted LJ potential with $\sigma=1.0 \sigma$ and $\varepsilon_{f f}=1.0 \varepsilon$. These param- 


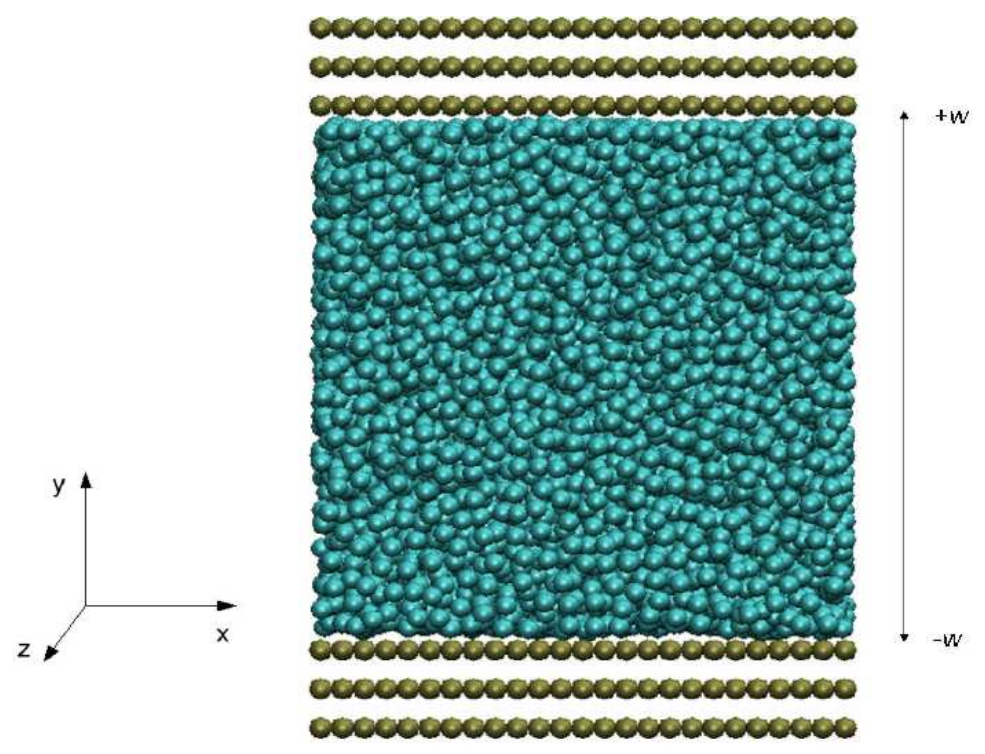

Figure 4.2: The $x y$ cross section of the simulation setup is shown here. The polymeric fluid (blue-coloured particles) is placed between atomistic walls (gold-coloured particles) at $\mp w$.

eters are fixed throughout all the simulations. The density of the melt was at $\rho_{f} \approx 0.9 \sigma^{-3}$. The mass of both fluid and wall particles were set to $1.0 \mathrm{~m}$.

We perform isothermal simulations where the temperature of the system was maintained at $T=1.0 k_{B} T / \varepsilon$ using the Langevin thermostat. Since the flow is in the $x$ direction and we are interested in the transverse flows $\left(u_{y}\right)$, thermostating these components can lead to undesirable results. Hence only the $z$ component of the equations of motion was thermostated to avoid any interference with the flow [110]. The Langevin thermostat provides us with the possibility of thermostating the individual components of equations of motion. Therefore, the equations of motion governing the system are given by

$$
\begin{aligned}
m \ddot{x}_{i} & =-\sum_{i \neq j} \frac{\partial \Phi_{i j}}{\partial x_{i}}, \\
m \ddot{y}_{i} & =-\sum_{i \neq j} \frac{\partial \Phi_{i j}}{\partial y_{i}}, \\
m \ddot{z}_{i}+m \Gamma \dot{z}_{i} & =-\sum_{i \neq j} \frac{\partial \Phi_{i j}}{\partial z_{i}}+f_{i},
\end{aligned}
$$

where $\Gamma=1.0 \tau^{-1}$ is the friction constant which represents the viscous damping due to the drag experienced by a fluid particle resulting from its motion through the Langevin solvent; and $f_{i}$ is the random force acting on particle $i$ that mimics the effect of random collisions the particle undergoes with the fictious solvent 
particles. Here $\tau=\sqrt{m \sigma^{2} / \varepsilon}$ is the characteristic time scale of the simulation. The equations of motion were integrated using the velocity Verlet algorithm with a timestep $\Delta t=0.001 \tau$. All the physical quantities are expressed in the reduced LJ units.

\section{Initial Configuration}

The initial configuration of the melt was generated by randomly placing polymer chains within the simulation domain i.e., between the walls discarding the excluded volume interactions. The bond length between monomers was set to $b \approx 0.97 \sigma$. Due to the random arrangement of the molecules in the simulation box, the initial melt consisted of highly overlapping monomers which is clearly unphysical. Therefore, a mechanism is required for which an equilibrated melt without any overlaps can be realized before starting the actual simulation.

There are several methods for which this can be achieved and we use the soft potential approach proposed by Auhl et al. [111]. In this method, the interaction between bonded monomers was modelled by the FENE potential in conjunction with the soft potential, while the non-bonded monomers interacted only via the soft potential. The soft potential is given by

$$
\Phi_{s o f t}(r)= \begin{cases}A\left[1+\cos \left(\pi r / r_{c S}\right)\right] & \text { for } r \leq r_{c S} \\ 0 & \text { for } r>r_{c S}\end{cases}
$$

where $r_{c S}=2^{1 / 6} \sigma$ is the cut-off radius for the interaction. The prefactor $A$ is the amplitude of the interaction energy whose value increases in time from 0.0 to 60.0 during the equilibration period of $50 \tau$. The systematic increase in $A$ ensures that the distance between overlapping particles increases in a gradual manner. The soft potential as a function of $A$ (or time) is shown in Fig. 4.3. Particles experience a large repulsive force at short distances and the magnitude of this force decreases with distance becoming negligibly small around $r \approx 1.0 \sigma$, which is approximately the inter-particle (between both bonded and non-bonded) distance. As the magnitude of $A$ increases in time so does the potential and this ensures a smooth transition of the system from a highly overlapping state to a well equilibrated melt.

During this equilibration period of $50 \tau$, the equilibration process was monitored by recording per monomer potential energies and the results are shown in Fig. 4.4. From this plot we can infer that the time period of $50 \tau$ is sufficiently long for the system to attain an equilibrium state. After this time period the interac- 


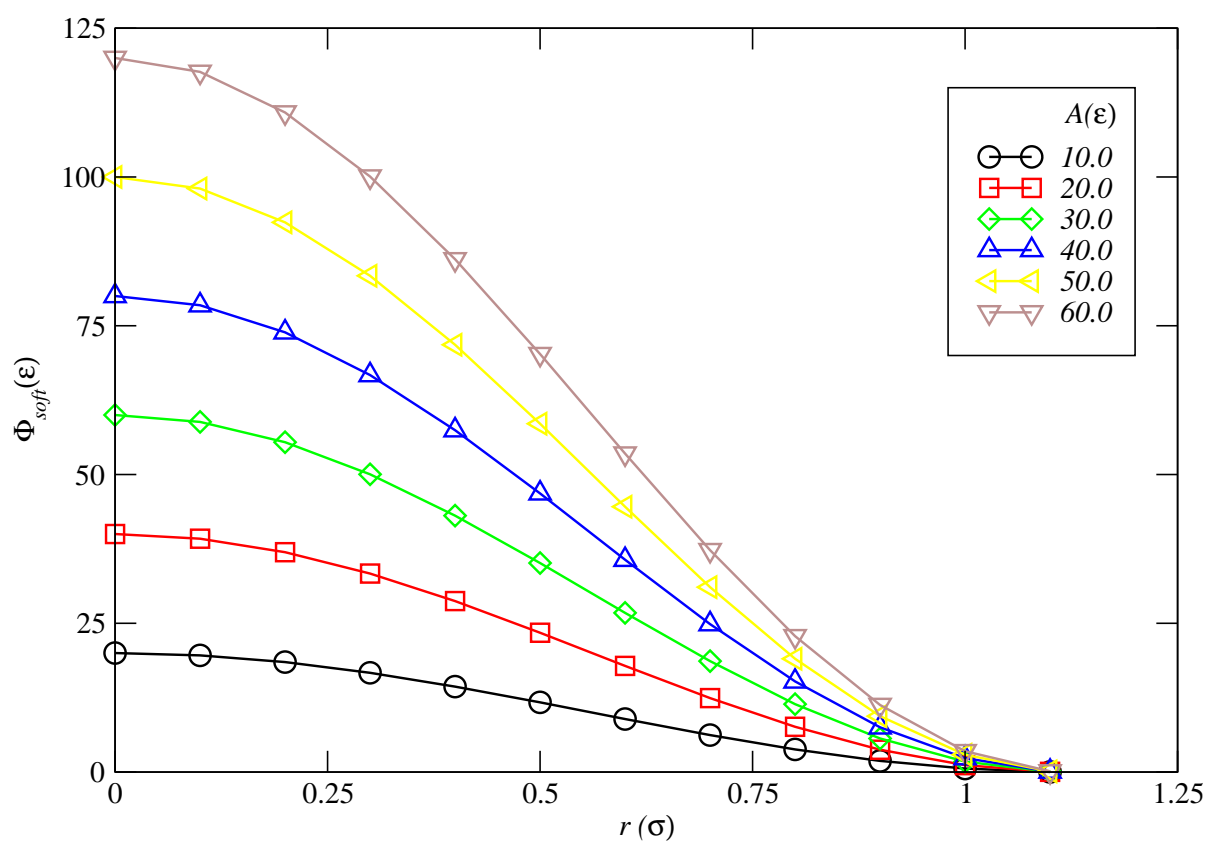

Figure 4.3: The soft potential as a function of distance $r$ for various interaction strengths $A$.

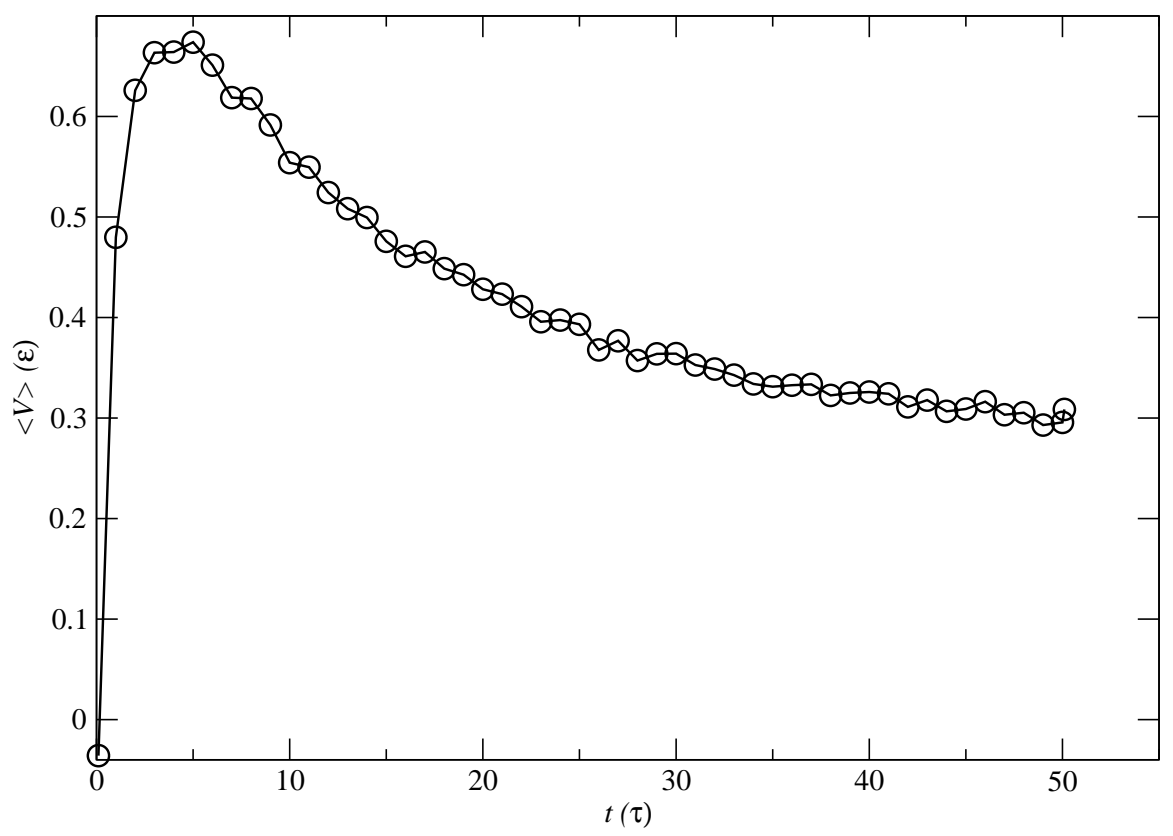

Figure 4.4: The average potential energy per monomer $\langle V\rangle$ as a function of time during the equilibration process. At initial times the potential energy sharply rises attributed to the overlaps in the system. After this time it starts to decline gradually and eventually attains a constant value around $40 \tau$.

tion between monomers (both bonded and non-bonded) was switched from the soft potential to the shifted LJ potential. Note the FENE potential between the bonded monomers stays intact. Further equilibration was carried out using the LJ potential, first for $50 \tau$ with $r_{c f f}=2^{1 / 6} \sigma$ then for another $50 \tau$ with $r_{c f f}=2.5 \sigma$. 
Here the subscript $c f f$ denotes the cut-off radius for monomer-monomer interaction in the LJ potential. This process was the first step for all the MD simulations polymer melts in this dissertation.

\subsection{Characterization of The Fluid}

Based on their rheological properties fluids can be categorized as simple or complex. Simple fluids obey Newton's law of viscosity which states that shear stress is proportional to the applied shear rate, $\Pi=\eta \partial u / \partial y$, where $\Pi$ is the shear stress. The constant of proportionality $\eta$ is known as the viscosity of the fluid and it is independent of applied shear rate. For complex fluids, on the other hand, the viscosity is not a constant but a function of applied shear rate and can also be time-dependent in some cases [56]. As a result of this, the stress versus shear rate response is non-linear, for complex fluids, which is why they are also called non-Newtonian fluids. Non-Newtonian fluids can be classified into three major categories:

- Pseudoplastic: The viscosity of the fluid decreases with increasing shear rate and attributing to this fact these fluids are also called shear-thinning fluids.

- Dilatant: Fluids, for which the viscosity increases with increasing shear rate are known as dilatant or shear-thickening fluids.

- Bingham plastics: These fluids demonstrate solid like behaviour below a critical shear stress and above which they start to flow with constant viscosity.

We now examine the viscous behaviour of the polymer melt under study using the Couette flow simulations.

\subsubsection{Couette Flow}

The equilibrated melt was obtained by using the same procedure as was discussed in Sec. 4.4. The LJ interaction parameter for the wall-fluid interaction was set to $\varepsilon_{w f}=1.0 \varepsilon$. The interaction between bonded monomers was described by the FENE potential. Both bonded and non-bonded monomers interacted via the LJ potential with $r_{c u t}=2.5 \sigma$.

The Couette flow was realized by applying a tangential velocity to the upper wall in the positive $x$ direction while the lower wall remained stationary. The velocity of the upper wall was increased in incremental steps of $\Delta U=0.1 \sigma / \tau$, until the wall velocity (or shear rate) has reached the desired value. After each increment of $\Delta U$, the system was equilibrated for $10 \tau$ to let it come to thermal equilibrium. Upon attaining the final value for the wall velocity, $U$, the system 
was equilibrated for another $500 \tau$ to reach steady state. This time period was found to be sufficiently long enough for the system to reach steady state. The steady state of the flow was confirmed by monitoring the potential energy, which attained a constant value during this period.

After reaching the steady state, we start making measurements on the system. Here we report some of the observations from these simulations. First, we compute the longitudinal velocity profiles as a function of shear rate. For this purpose, the simulation domain (between the walls) was divided into bins of length $\Delta y=0.2 \sigma$ in $y$ direction. At each timestep, fluid particles were mapped into one of these bins based on their $y$ coordinates. Then an average velocity for each bin was evaluated as:

$$
\left\langle u_{x}(y, y+\Delta y)\right\rangle=\frac{\sum_{i=1}^{n} u_{x i}}{n},
$$

where the summation is over all the particles $n$ which lie between $y$ and $y+\Delta y$. To minimize thermal noise further time averaging of this quantity was done for $10^{3} \tau$. The thus obtained tangential velocity profiles for different $U$ are shown in Fig. 4.5. The magnitude of the velocity profiles was found to be proportional to

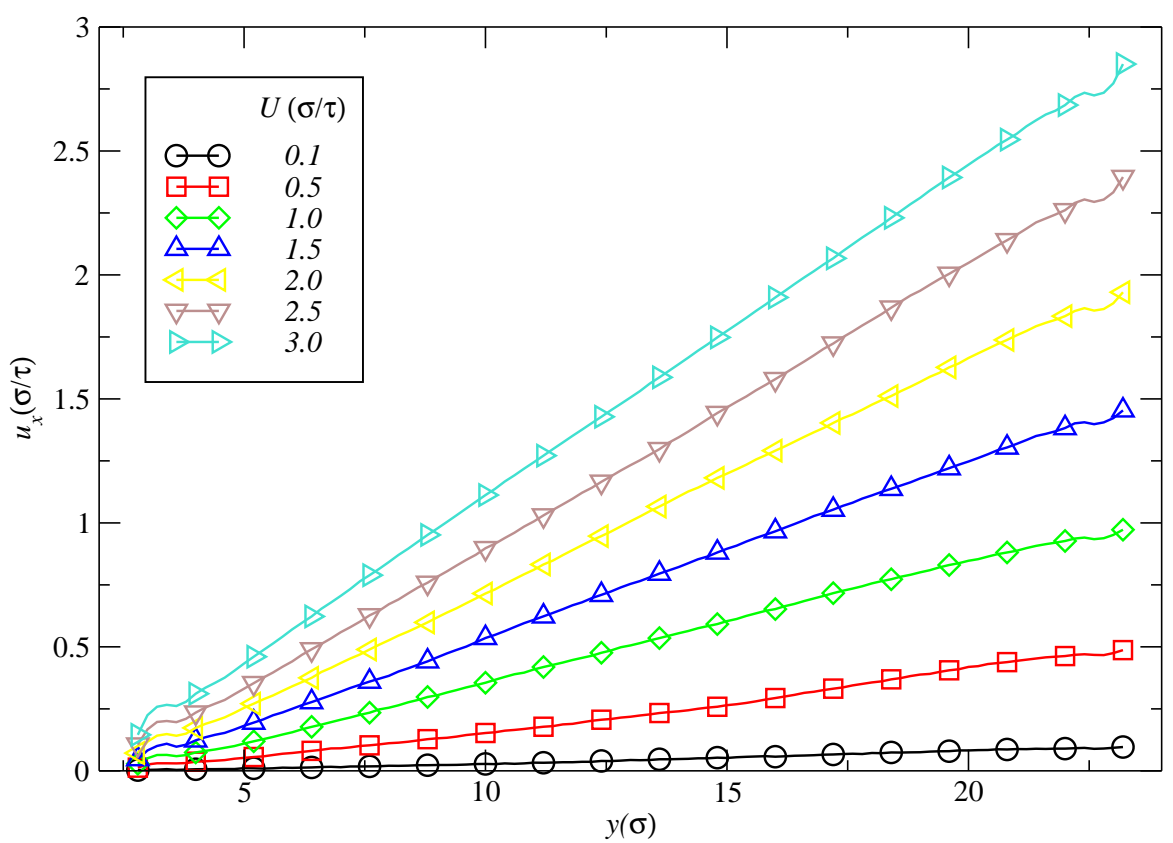

Figure 4.5: Longitudinal velocity profiles for different upper wall velocities $U$. The velocity profiles are linear except for slight curvature up to a distance of $\sigma$ away from both inner wall surfaces. The magnitude of the velocity profiles is linearly proportional to $U$.

the applied shear rate $(\dot{\gamma}=U / 2 w$, where $2 w$ is the separation between the walls (See Fig. 4.2)). 
A finite slip was noticed close to both walls. Respective slip lengths were extracted by doing a linear extrapolation of $u_{x}(y)$ into the wall and the distance at which $u_{x}(y)$ become zero was identified as the slip length at that particular shear rate. The results are shown in Fig. 4.6. The trend in the slip length behaviour,

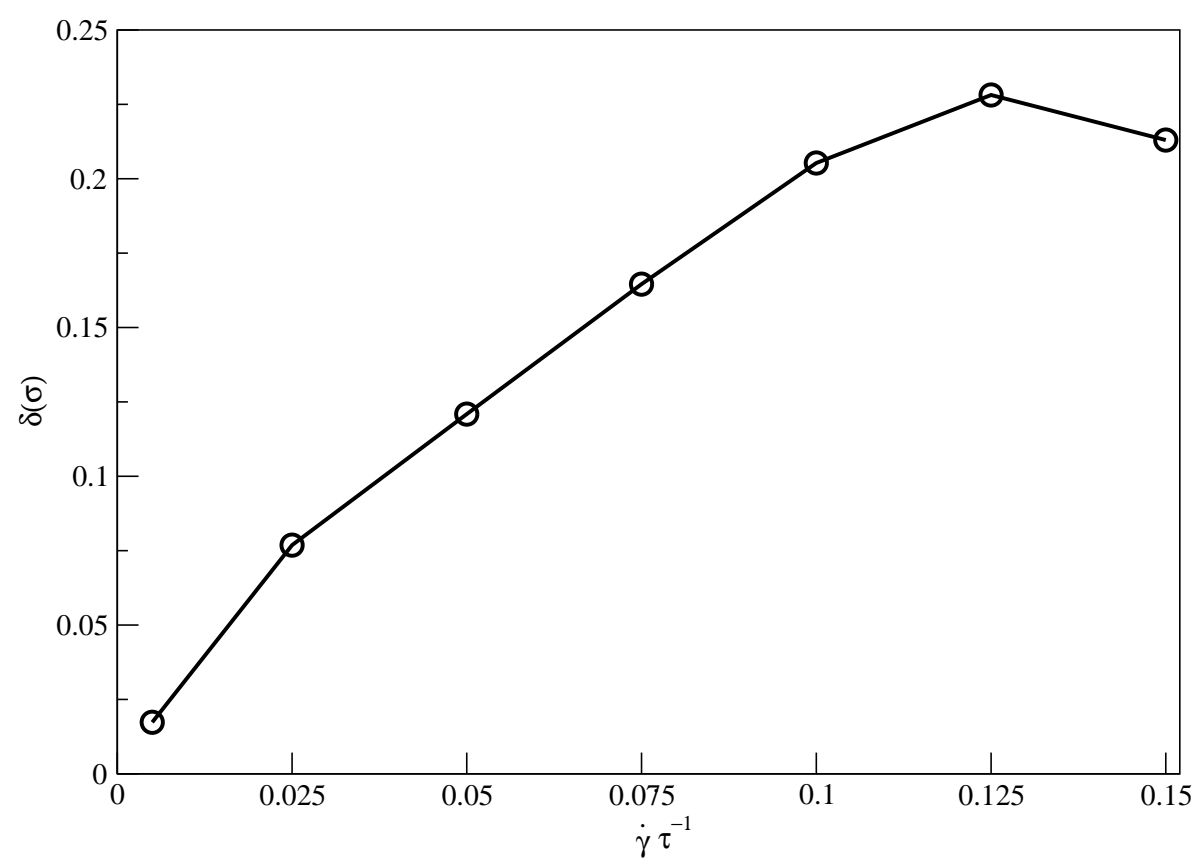

Figure 4.6: The slip length as a function of shear rate.

as a function of shear rate, is in qualitative agreement with the previous studies $[108,112,113]$. However, a quantitative comparison is not possible as they were carried out under different conditions. The shear flow simulations are often criticized for using unrealistically high shear rates at which the heat generated per volume is quite large. Our calculations show that this is not the case for polymer liquids i.e., the shear rates used are reasonable. Moreover, through our simulations we are attempting to predict the fluid behaviour under the given setup rather than realizing the experimental conditions.

The longitudinal velocity profiles in Fig. 4.5 display a slight curvature in the region $\sigma$ away from the walls. This was due to the layering of particles near the wall surfaces. To demonstrate this we plot the density and velocity profiles for $U=2.5 \sigma / \tau$ in Fig. 4.7. The density profile has primary peaks nearly $\sigma$ away from both walls. Close to the stationary wall the velocity profile has a bump and deviates from linearity. In the same region, the density profile reveals the existence of the first fluid layer and thus more fluid particles exist in this region. This region is followed by a region with fewer particles (the first valley in Fig. 4.7) and thus a drop in velocity. After this point the fluctuations in density die out and the 


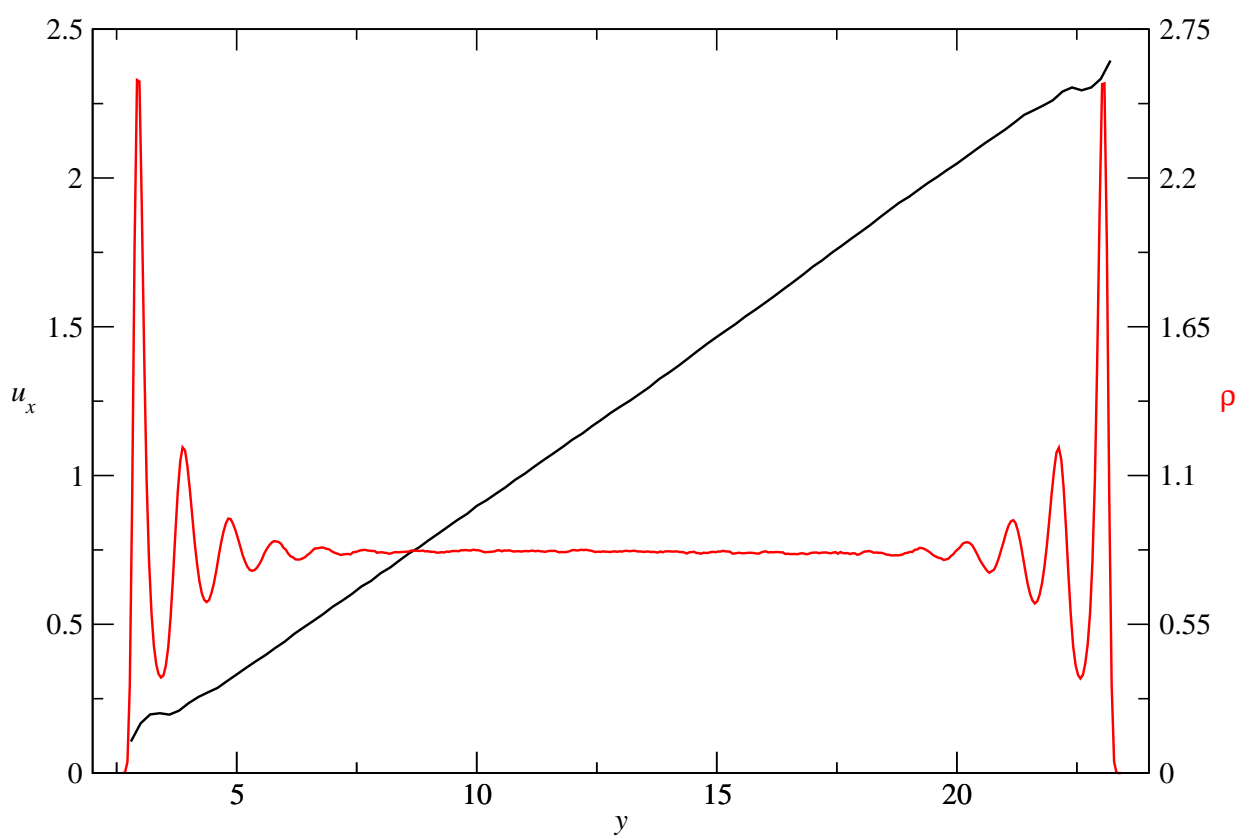

Figure 4.7: Density and $u_{x}$ component of velocity are plotted as a function of $y$. The black line represents the velocity profile; the red line is the density profile.

velocity profile becomes linear. Similar analysis can be applied to explain the deviation from linearity in the velocity profile near the upper wall. However, close to the upper wall the minimum density has a re profound effect on the velocity profile (dip in velocity) than the primary maximum. This could be due to the strong influence of the upper wall movement on the fluid, dragging along fluid layers with it.

The shear viscosity is defined as

$$
\eta=\frac{\Pi_{x y}}{\dot{\gamma}}
$$

where $\Pi_{x y}$ is the $x y$ component of the stress tensor. We calculate $\Pi_{x y}$ based on atomistic implementation of the Irving-Kirwood method [114, 115]:

$$
\Pi_{x y}=-\frac{1}{V}\left\langle\sum_{i=1}^{N} m_{i} u_{i x} u_{i y}+\sum_{i=1}^{N} \sum_{j>i}^{N} r_{i j x} F_{L J_{i j y}}+\sum_{i=1}^{N_{c}} \sum_{j>i}^{N_{m}} r_{i j x} F_{b o n d_{i j y}}\right\rangle,
$$

where $V, N, N_{C}$ and $N_{m}$ are the system volume, total number of particles in the system, number of chains and the chain length, respectively and $\langle\cdots\rangle$ represents the time average of the quantity over the simulation period. The first term in the above equation is the kinetic energy contribution to the stress tensor; $u_{i x}$ and $u_{i y}$ are the $x$ and $y$ components of the velocity of particle $i$, respectively. The second term is the potential energy contribution coming from the pair-wise LJ interac- 
tions where $r_{i j x}$ and $F_{L j_{i j y}}$ are the $x$ component of the displacement vector and the $y$ component of the LJ force, between particles $i$ and $j$, respectively. The third term accounts for bond interactions where $F_{b o n d_{i j y}}$ represents the $y$ component of the bond force between particles $i$ and $j$. For each shear rate, the stress tensor component $\Pi_{x y}$ was calculated using Eq. (4.7) and the corresponding shear viscosity was calculated using Eq. (4.6). The results are shown in Fig. 4.8. At

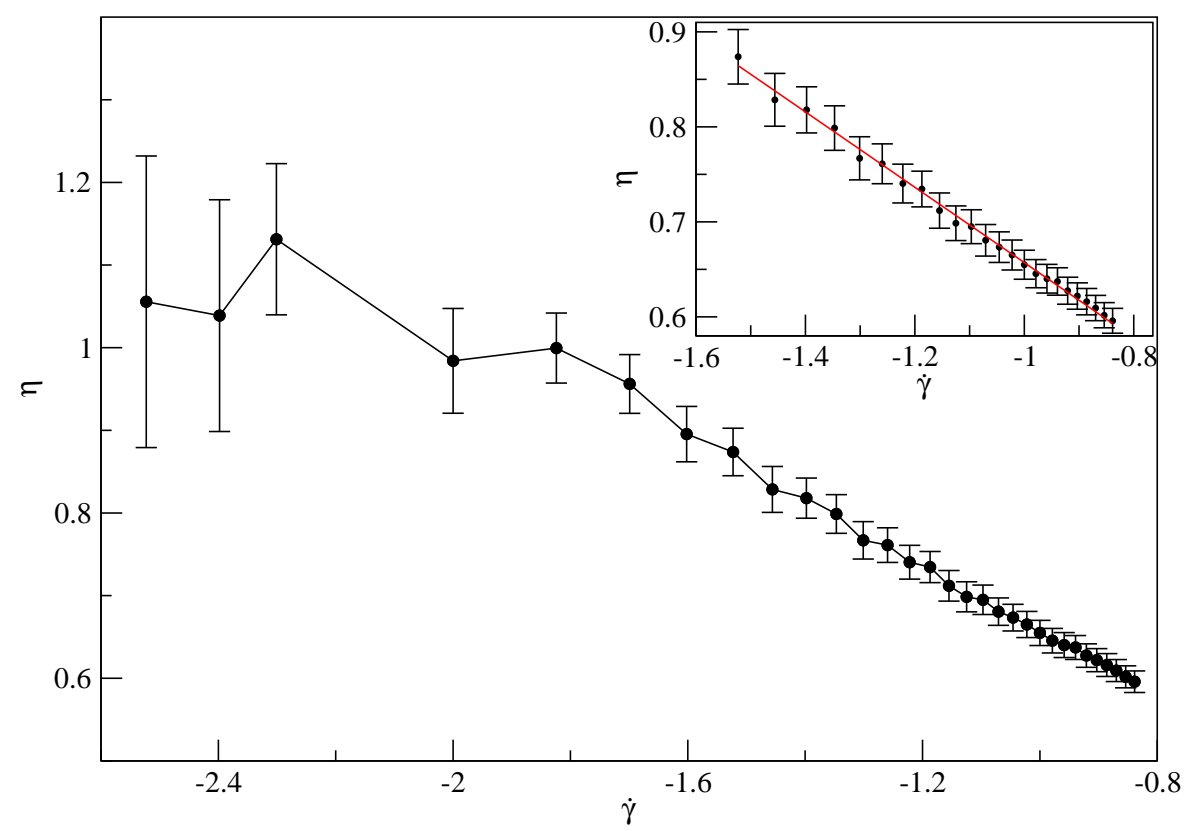

Figure 4.8: Log-log plot between shear viscosity $\eta$ and shear rate $\dot{\gamma}$. The figure in the inset is the viscosity response in the high shear rate limit. The red line is the line-fit to the viscosity in this regime.

low shear rates, the fluctuations in viscosity are quite large. This is because at these shear rates, the thermal velocity of the fluid is greater than the velocity of the characteristic flow thus leading to large errors in the measured viscosity $[113,116,117]$. At large shear rates, we fit these data with the power law model [56] for non-Newtonian fluids according to which viscosity of the fluid is given by $\eta=m(\dot{\gamma})^{n-1}$, here $m$ is the consistency coefficient and the exponent $n$ determines the nature of the fluid. A value of $n<1$ means the fluid is shear-thinning and $n>1$ corresponds to a shear-thickening fluid. The model fits the data very well in the high shear rate limit (See the inset figure in Fig. 4.8) and by measuring the gradient of this line-fit we extract the exponent $n$ to be $n=0.6063 \pm 0.0138$ suggesting that the fluid under consideration is shear-thinning. The value of the consistency coefficient $m$ was found to be 1.84 . 


\subsection{Determining the Slip Lengths}

The slip length of a fluid at the solid boundary is strongly influenced by the interaction between them. In our simulations, we control the slip length by adjusting the wall-fluid interaction parameter $\varepsilon_{w f}$ in the LJ potential. A series of Poiseuille flow simulations with different $\varepsilon_{w f}$ were carried out. From the resultant velocity profiles the slip lengths $(\delta)$ corresponding to the particular $\varepsilon_{w f}$ were extracted.

In experiments, Poiseuille flows are realized by applying a constant pressure gradient across the channel ends. However, insofar as simulations are concerned, pressure gradients can lead to inhomogeneities in the fluid [118]. Poiseuille flows can also be realized by the application of constant body force on each of the fluid particles, and are much simpler to implement in simulations. A very detailed and insightful discussion on this topic can be found in the article by Todd et al. [118].

Except for the interaction parameter between the fluid and the wall, $\varepsilon_{w f}$, all other interactions are the same as in the previous section. By varying $\varepsilon_{w f}$, a range of slip lengths could be studied. For each $\varepsilon_{w f}$, the system was initially equilibrated for $100 \tau$. After this period, a body force was applied, on each monomer every timestep, in incremental steps of $\Delta F=0.01 \mathrm{m \sigma} / \tau^{2}$ and the system was equilibrated for $10 \tau$ at each increment to stabilize the temperature of the fluid. Upon reaching the desired value of $F=0.05 \mathrm{~m} \sigma / \tau^{2}$, the fluid was further equilibrated for another $10^{3} \tau$ to let the flow attain steady state. Now we start computing the longitudinal velocity profiles by dividing the system into bins of size $\Delta y=0.2 \sigma$ along $y$ direction. At each timestep, the fluid particles were mapped into one of these bins depending on their $y$ coordinates. An average velocity $\left\langle u_{x}(y)\right\rangle$ was calculated for each bin using Eq. (4.5). Thus obtained bin velocities were further averaged over time for $2 \times 10^{3} \tau$, for more accurate results. The longitudinal velocity profiles for different $\varepsilon_{w f}$ values are shown in Fig. 4.9. It is quite evident from Fig. 4.9, that fluid experiences a finite slip at the wall-fluid boundary (since $\left.u_{x}(\mp w) \neq 0\right)$.

To extract slip lengths from these velocity profiles, we fit them with the theoretical predictions. The fluid flow can be described using the Navier-Stokes equations. The continuity equation for the mass conservation:

$$
\frac{\partial \rho}{\partial t}+\nabla \cdot(\rho \mathbf{u})=0
$$

Assuming that the fluid is incompressible and flow has reached the steady state 


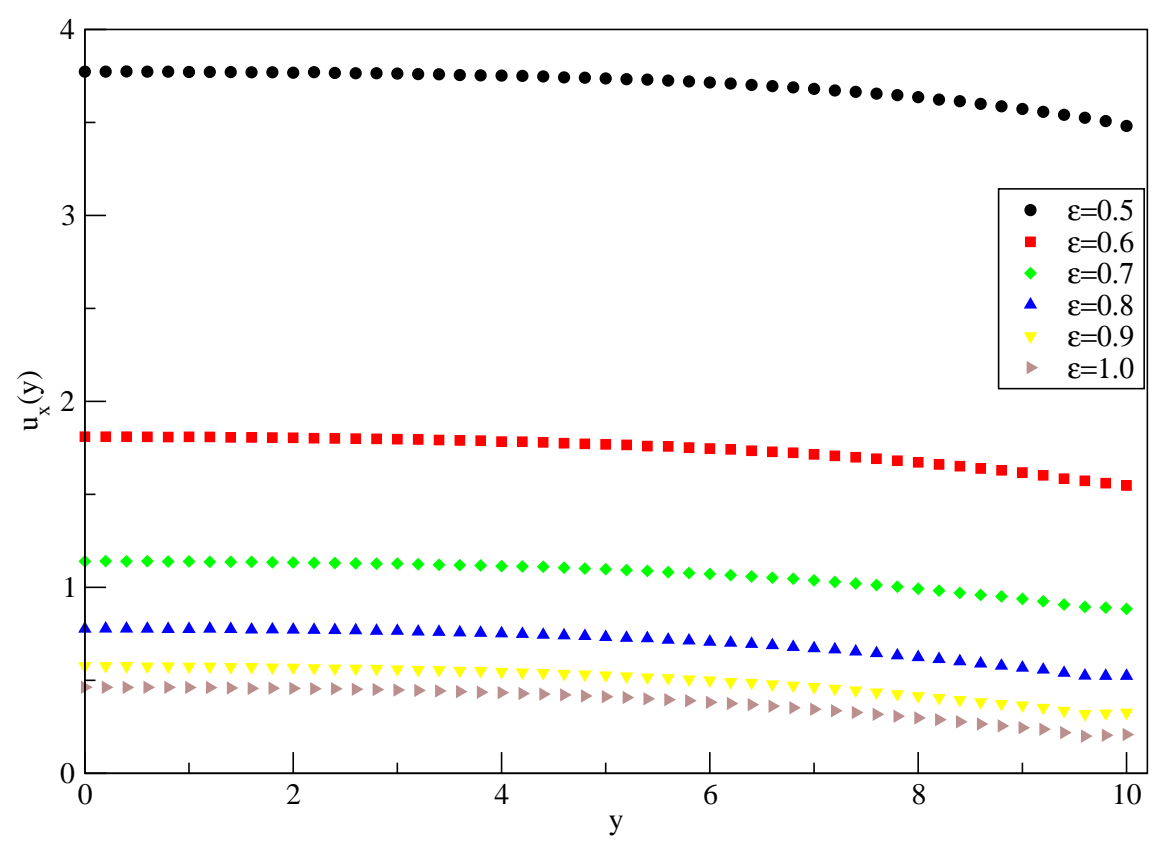

Figure 4.9: Average longitudinal velocity profiles for different values of $\varepsilon_{w f}$ for $y \geq 0$. Note, the velocity profiles are symmetric about $y=0$.

the first term in the Eq. (4.8) drops out and the equation reduces to

$$
\nabla \cdot \mathbf{u}=\mathbf{0}
$$

The equation for the conservation of momentum is given by

$$
\rho\left(\frac{\partial \mathbf{u}}{\partial t}+\mathbf{u} \cdot \nabla \mathbf{u}\right)=-\nabla \cdot \underline{\underline{\Pi}}+\rho \mathbf{F}
$$

where $\underline{\underline{\Pi}}$ is the stress tensor and $\mathbf{F}$ is the body force acting on the fluid. The Navier-Stokes solution that best represents our system is two dimensional in $(x, y)$ since the simulation domain is periodic in the $z$ direction. Since the flow is laminar at these length scales $(\sim 20 \sigma-40 \sigma)$ and symmetric in the $z$ direction, only the $u_{x}$ component of the velocity, which is the velocity in the direction of the force, is non-zero. Furthermore, we must have $u_{x}=u_{x}(y)$ since we have $u_{y}=0$. Assuming that the flow has attained steady state, the left hand side of Eq. (4.10) is zero since the flow is incompressible and laminar. To solve for the velocity field $u_{x}(y)$ in Eq. (4.10) we implement the Navier slip boundary condition [96]. According to this condition the longitudinal component of the fluid velocity at the solid boundary:

$$
u_{x}( \pm w)=\mp \delta \frac{\partial u_{x}}{\partial y}( \pm w)
$$

where $\delta$ is the slip length. Solving Eq. (4.10) using the Navier boundary condition 
yields

$$
u_{x}(y)=\left(\frac{F w \rho}{m}\right)^{1 / n}\left[\frac{w}{1+1 / n}\left\{1-\left(\frac{y}{w}\right)^{1+1 / n}\right\}+\delta\right] \quad \text { for } y \geq 0
$$

where the values of $m$ and $n$ are available from the Couette flow simulations. Note that $u_{x}(y)$ is symmetric about $y=0$. All the quantities in Eq. (4.12) are known except for the slip length $\delta$. By fitting Eq. (4.12) to the velocity profiles in Fig. 4.9 we obtain the relationship between $\varepsilon_{w f}$ and $\delta$. The effect of interaction parameter $\varepsilon_{w f}$ on the slip length $\delta$ is shown in Fig. 4.10. The slip length was found to decrease with increasing $\varepsilon_{w f}$. A large $\varepsilon_{w f}$ implies that monomers will be strongly attracted to the wall and consequently will tend to stick to it, leading to a small slip length $\delta$.

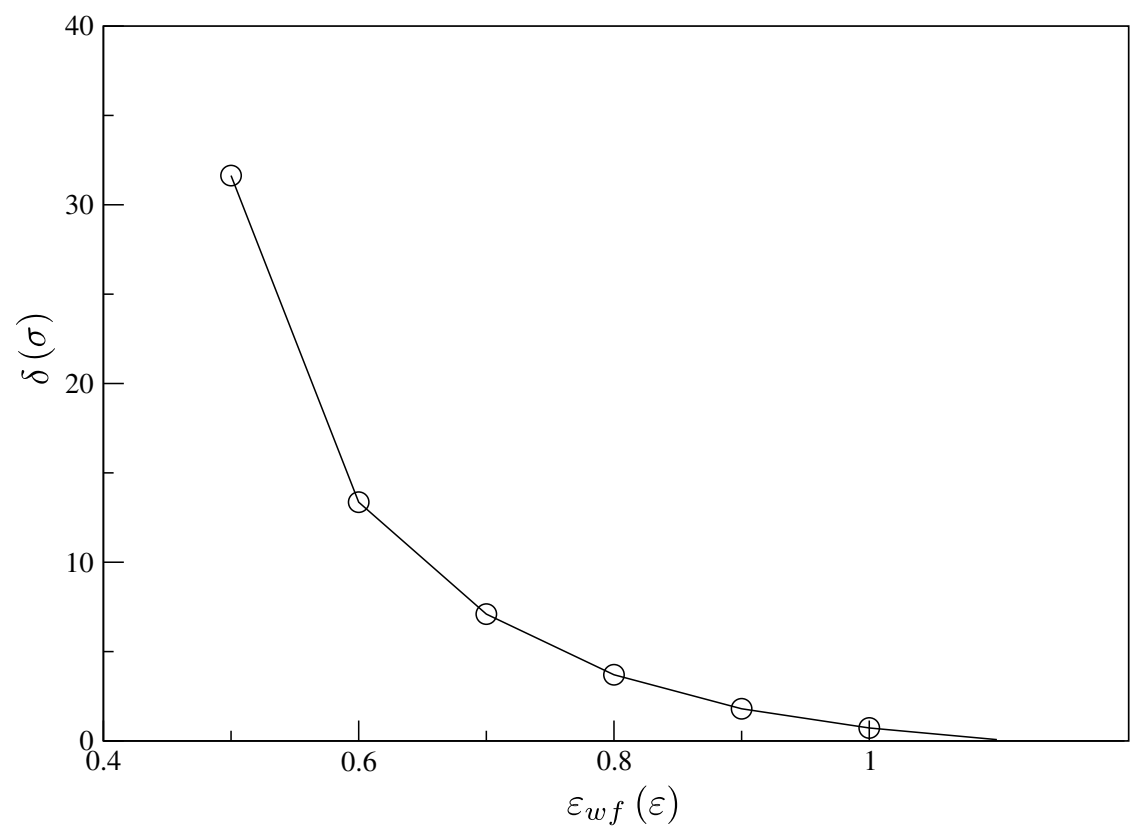

Figure 4.10: Slip length $\delta$ as a function of the interaction parameter $\varepsilon_{w f}$.

\subsection{Simulations for Enhanced Mixing}

\subsubsection{Patterned Boundaries}

We now study the effect of patterned-slip boundary conditions on the fluid flow and try to quantify its effect on the mixing behaviour. The patterned-slip boundary conditions were implemented by creating alternate stripes of high slip (low wettability) and low slip (high wettability) along the channel walls in the $x$ direction. In our simulations this was realized by varying the wall-fluid inter- 
action $\varepsilon_{w f}$ of the LJ potential in these regions, along the walls in the $x$ direction. Using this strategy we created two types of wall atoms, $A$ and $B$, with $\varepsilon_{w f_{1}}=0.5 \varepsilon$ and $\varepsilon_{w f_{2}}=0.9 \varepsilon$, representing their respective interaction strengths with fluid particles. A schematic diagram describing this setup is shown in Fig. 4.11. As
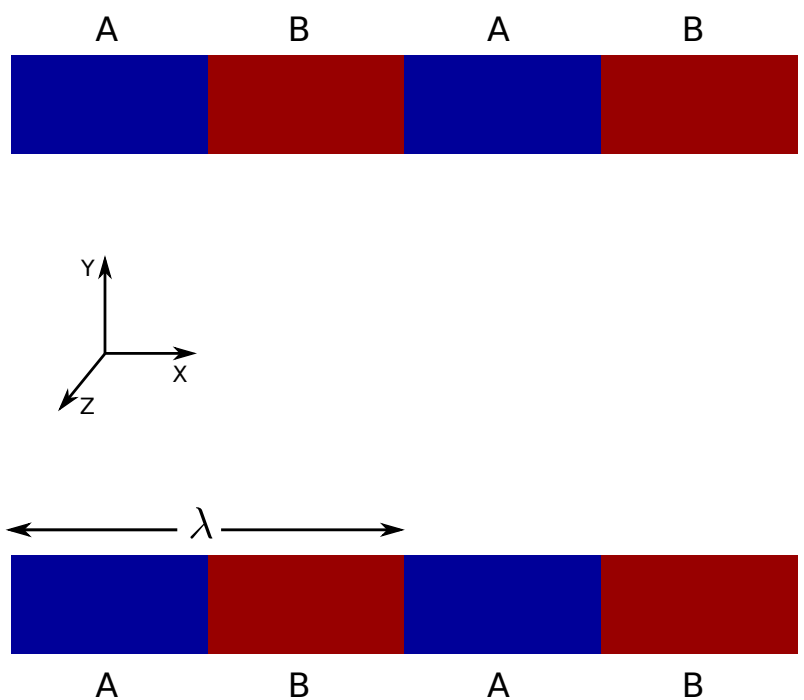

Figure 4.11: Schematic diagram of the pattern slip boundary setup for MD simulations. The blue colour represents A-type wall atoms and the red colour represents B-type wall atoms. The block lengths of A and B regions are equal in size. $\lambda$ is the wavelength of the pattern.

a consequence, fluid particles experience a large slip when they interact with Atype wall atoms and a small slip when they interact with B-type wall atoms (See Fig. 4.10).

To start with once again the polymer molecules were placed between the channel walls and the system was equilibrated as described in Sec. 4.4, but this time with patterned-slip boundary in place as shown in Fig. 4.11. After this equilibration period, the body force was ramped up to $F=0.05 m \sigma / \tau^{2}$ as described in the previous section. As pointed out previously, one of the indications of mixing is the presence of transverse flows. Here we compute transverse velocity profiles $u_{y}(x, y)$ for various patterned-slip boundary conditions, to study the mixing properties. The contour plots for the transverse velocity were calculated by dividing the simulation domain into bins of size $\Delta x \Delta y=1.0 \sigma^{2}$ and at each timestep particles were mapped into one of these bins. The average transverse velocity $u_{y}(x, y)$ for each of these bins was obtained by dividing the total transverse velocity due to all the particles in the bin by the number of particles that contributed to the sum. For better statistics, time averaging of this profile was performed over the next $4 \times 10^{3} \tau$. This time period for averaging was found to be sufficiently long enough as the transverse profiles with longer averaging periods do not show any 
significant deviations.

Simulations were carried out for different pattern wavelengths and their effect on the transverse velocity was observed. The wavelength $\lambda$ (or wave number $k=2 \pi / \lambda)$ of the pattern can be varied by changing the A plus B block length (See Fig. 4.11). Simulations were conducted for wavelengths $k w=\pi / 2,2 \pi / 3$, $\pi, 4 \pi / 3$ and $2 \pi$ and corresponding time averaged $u_{y}(x, y)$ profiles are shown in Fig. 4.12. We observe alternating near circular regions of high positive and negative transverse velocities in both upper and lower halves of the $x y$ cross section about the channel axis, along the $x$ direction. The number of alternating patterns in the transverse profile is equal to the number of patterns along the channel wall. However, the velocity profiles did not align with the wall pattern. This is due to the inertia of the fluid and hence the phase lag between the applied force and the transverse velocity profiles. This observation is consistent with the continuum theory which suggests a phase lag of $\pi / 2$ between the wall pattern and transverse velocity response $[25,1]$. The transverse velocity profiles were anti-symmetric about the channel axis. That is, if the upper half has a positive transverse velocity region then the lower half of the same region has a negative transverse velocity region. The profile pattern was reversed in the subsequent patterned region. It is evident that the patterned-slip boundary induces transverse flows.

The curl of a velocity field is the measure of rotation of the fluid. This quantity is known as the vorticity and it is defined by $\Omega \equiv \nabla \times \mathbf{u}$. We compute the vorticity for the case $k w=\pi / 2$ and the resulting contour plot is shown in Fig. 4.13. Note, the dominant Poiseuille velocity has been subtracted from $\mathbf{u}$ to obtain this plot. The vorticity is (approximately) symmetric about the $x$ axis. Consider the domain $x>0$. In this domain the maximum magnitude of the vorticity occurs in the region between the maximum magnitude of the transverse velocity. This is in agreement with the expectations from continuum theory [1]. Along the axis of the channel the vorticity is small as in these regions the flow is predominantly in the longitudinal direction.

One important scaling factor in this study is the size of the polymer to the wavelength of the wall-slip pattern. Its effect is evident from Fig. 4.12 where transverse velocity profiles for different pattern wavelengths are plotted. To understand these profiles and the variations in the magnitude of transverse velocity profiles we calculate the average size of the polymer molecule, the radius of gyration $\left(R_{g}\right)$, in the melt. Using the Poiseuille flow simulations with constant slip boundary condition, achieved by setting wall-fluid interaction to $\varepsilon_{w f}=1.0 \varepsilon$, we compute the average radius of gyration of the chain to be $R_{g}=5.369 \sigma$. Com- 


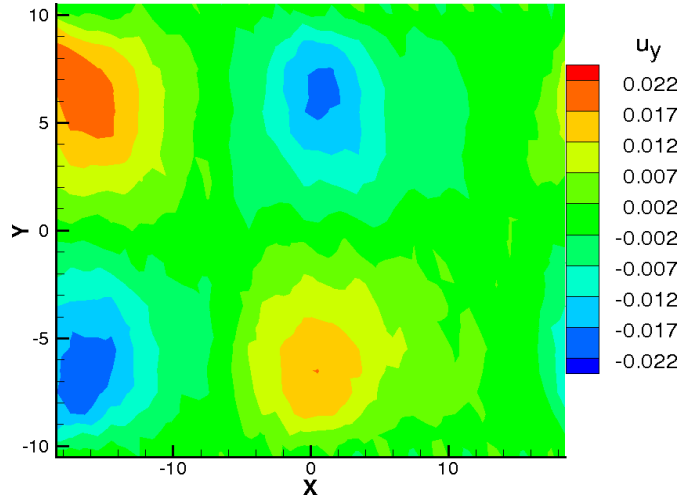

(a) $k w=\pi / 2$

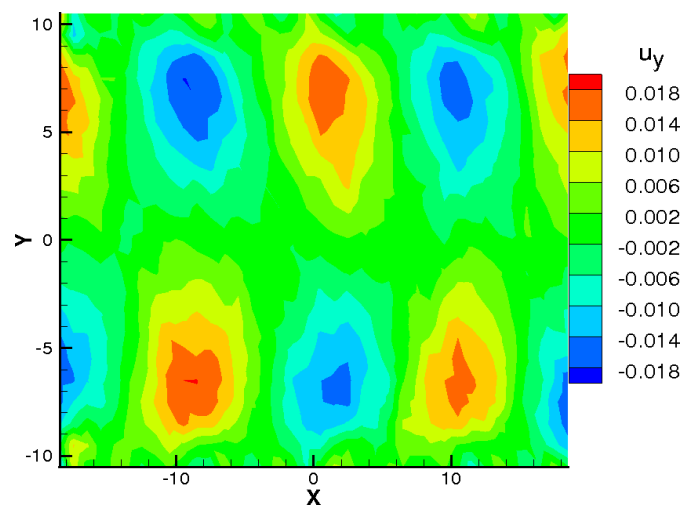

(c) $k w=\pi$

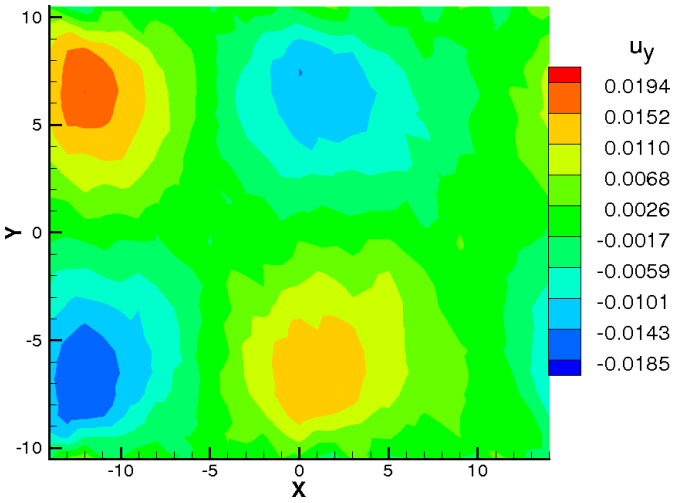

(b) $k w=2 \pi / 3$

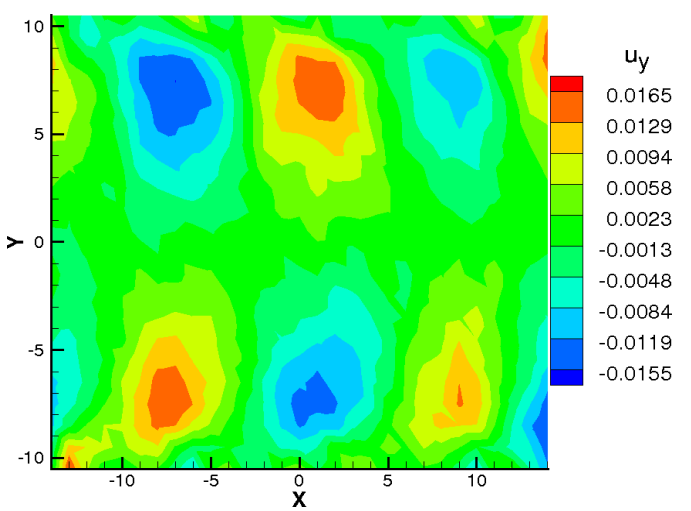

(d) $k w=4 \pi / 3$

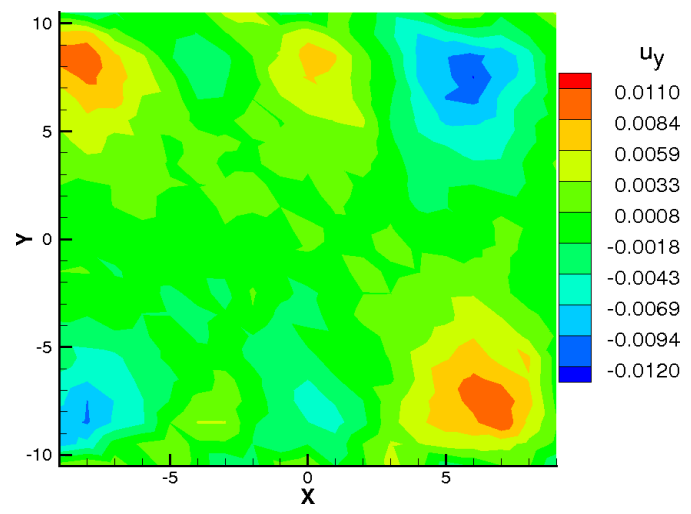

(e) $k w=2 \pi$

Figure 4.12: Contour plots of the transverse velocity profile $u_{y}(x, y)$ for various $k w$. Red regions represent positive transverse velocity regions and blue regions represent negative transverse velocity regions.

parison between $R_{g}$ and $\lambda$ helps us understand the transverse velocity profiles. Well developed transverse flows were observed when the radius of gyration of the polymers $\left(R_{g}\right)$ was smaller than the pattern length $(\lambda / 2)$. However, in the case of $k w=2 \pi$ the pattern length was $4.8 \sigma$, which is less than the radius of gyration of the polymers. In this case the transverse flows were suppressed. The ratio of the length of the patterned region to the radius of gyration of polymer chains seems to be an important factor in determining the amount of transverse 


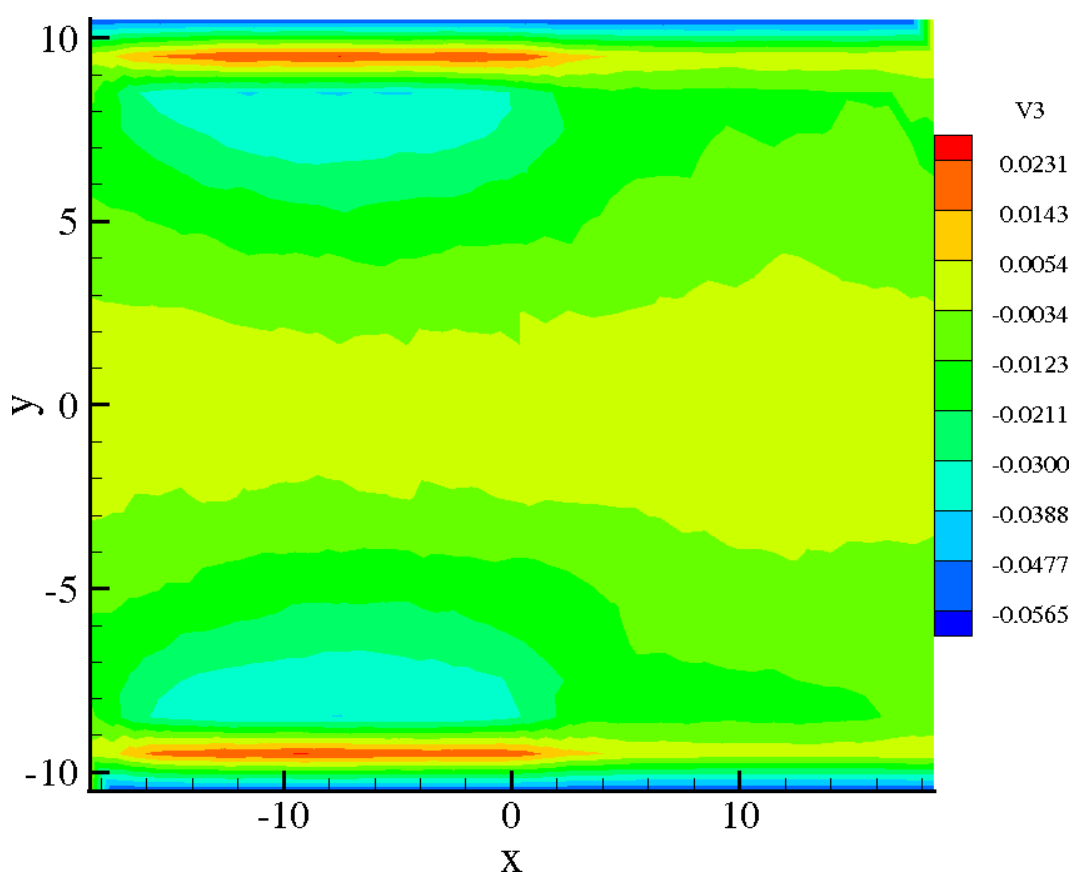

Figure 4.13: The vorticity field for the case $k w=\pi / 2$. Here the magnitude of the vorticity is indicated by label V3. Note, maximum vorticity occurs in the region between maximum magnitude of transverse velocity.

flow that can be achieved. The physical origins of this effect can be understood in the following way. When the length of a patterned region is comparable to the radius of gyration of the polymer chains then, on average, a polymer chain spans two different wettable wall regions simultaneously. Hence these chains start interacting with the wall as if it was a homogeneous continuum, thus decreasing the transverse flow. In our simulations for $k w=2 \pi$, the length of a patterned region was less than the radius of gyration of polymer chains and we have noticed a significant drop in the transverse velocity. The magnitude of the maximum transverse velocity in this case decreased by a factor of 2 compared to the magnitude of the maximum transverse velocity for $k w=\pi / 2$. We have also observed that the transverse flow was not as well developed as in the other cases where the $R_{g}$ was less than the pattern length. This indicates that the length of the patterned regions should be longer than radius of gyration to enhance transverse flows. This is an important result, which is not captured by the continuum modelling [1] since it does not contain information regarding individual chains and hence does not include the length-scale $R_{g}$. Alternatively, putting these two results together leads us to a relationship between channel width and radius of gyration for maximum transverse velocity, i.e. $w>R_{g} / 2$. Of course, the upper limit on the channel width is given by (external) physical restrictions. 


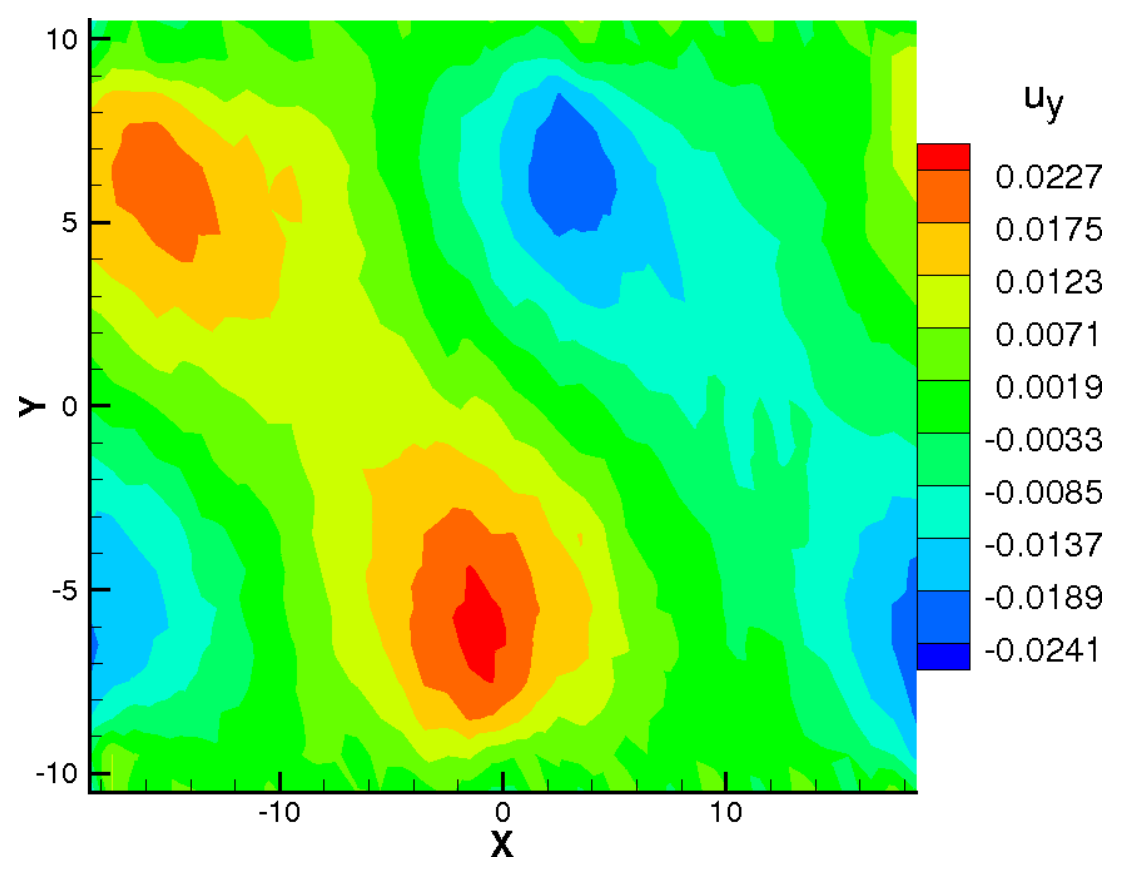

Figure 4.14: Contour plot of transverse velocity profile $u_{y}(x, y)$ for $k w=\pi / 2$ with offset patterning.

\subsubsection{Off-Set Patterning}

Simulations with off-set patterning of the walls were also conducted to study their effect on the transverse flow. The off-setting was done by moving the upper wall patterning by $3.2 \sigma$ to the right while the lower wall's patterning position remained unchanged. We refer to the earlier case where the patterns were exactly parallel to each other as parallel patterning. Results of the simulations with offsetting in the pattern have shown no significant deviations in the magnitude of the maximum transverse velocity compared to that of parallel patterning. However, the transverse velocity regions in the upper and lower halves of the crosssection do not align parallel to each other as in the case of parallel patterning but they are off-set (see Fig. 4.14).

This was expected since the off-setting in the patterning leads to different flow behaviour on either side of the channel axis and hence off-setting of transverse flow regions occurs. An interesting observation from these simulations is that the transverse velocity is non-zero, in certain regions, in the centre of the channel. Hence, with this patterning setup fluid elements in the centre of the channel can flow towards the boundaries. In contrast, in the parallel patterning case, the transverse fluid velocity in the centre of the channel was always close to zero and thus fluid elements in centre of channel would never mix (except through diffusion). Therefore we suggest to obtain mixing throughout the channel, it is better 
to have off-set patterned regions on opposite sides of the channel.

\subsubsection{Comparison with Continuum Modelling}

We compare results obtained from the MD simulations with continuum modelling calculations based on Pereira's [1] perturbation analysis. This method is only applicable in the limit where the variation in slip length is small. In the continuum modelling the Navier-Stokes equations for the flow were solved using the Navier slip boundary condition. The patterned-slip boundary condition was implemented by defining the slip as a function of $x$ :

$$
\delta=\delta_{0}\left(1+\alpha e^{i k x}\right)
$$

where $\alpha$ is a small perturbation about the mean value of slip length $\delta_{0}$. Because of the variation in the slip boundary conditions both $u_{x}$ and $u_{y}$ must be functions of $x$ and $y$. Using the perturbation analysis the longitudinal and transverse velocity profiles can be written as

$$
\begin{aligned}
& u_{x}=u_{x_{0}}+\alpha u_{x_{1}}+O\left(\alpha^{2}\right)+\cdots, \\
& u_{y}=\alpha u_{y_{1}}+\cdots,
\end{aligned}
$$

where $u_{x_{0}}$ is given by Eq. (4.12). Thus solving the Navier-Stokes equations with the above conditions yields

$$
\left|u_{y}\right|=\alpha \psi R
$$

where

$$
\psi=w\left(\frac{F w \rho}{m}\right)^{1 / n}
$$

and $R$ is given by the solution of the following differential equation

$$
\begin{aligned}
& \bar{y}^{2} \frac{d^{4} R}{d \bar{y}^{4}}+2(1-1 / n) \frac{d^{3} R}{d \bar{y}^{3}}+2\left[\bar{y}^{2}(k w)^{2}(1-2 / n)-(1-1 / n) / n\right] \frac{d^{2} R}{d \bar{y}^{2}} \\
& +2(1-1 / n)(1-2 / n)(k w)^{2} \frac{d R}{d \bar{y}}+(k w)^{2}\left[(k w)^{2} \bar{y}^{2}-(1-1 / n) / n\right] R=0
\end{aligned}
$$

where $\bar{y}=y / w$. The boundary conditions for the above equation are:

$$
\begin{aligned}
& R(0)=0 \\
& R(1)=0
\end{aligned}
$$




$$
\begin{aligned}
R^{\prime \prime}(0) & =0 \\
R^{\prime}(1)+(\delta / w) R^{\prime \prime}(1) & =(\delta / w)(k w)
\end{aligned}
$$

This analysis cannot be applied to verify our existing MD results as it is only valid for small $\alpha$. To accomplish a reasonable comparison between the theory and simulations, we carried out simulations corresponding to a small $\alpha$. From Fig. 4.10, the choice of optimal $\varepsilon_{w f}$ values were found to be $\varepsilon_{w f_{1}}=0.8 \varepsilon$ and $\varepsilon_{w f_{2}}=0.9 \varepsilon$. We conducted MD simulations with these interaction parameters for the case $k w=\pi$ and found the maximum transverse velocity to be $u_{y_{\max }}=0.007 \sigma / \tau$. Note, for the MD simulations, patterning of the boundaries was implemented as described in the Sec. 4.7.1 where we have square-wave variation in the slip length where as the continuum theory is based on a sinusoidal variation. However, Hendy et al.[25] showed this does not influence transverse flows significantly as long as $\alpha$ is sufficiently small.

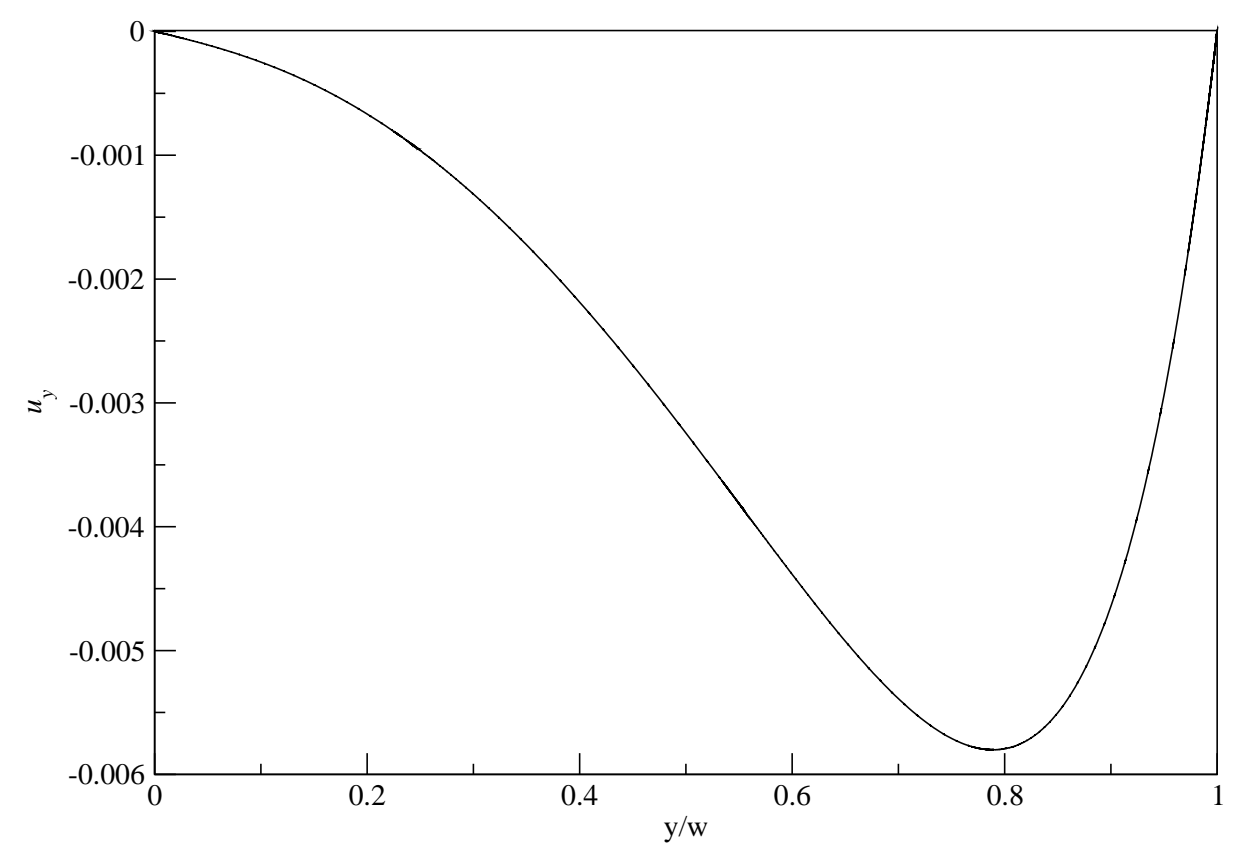

Figure 4.15: The transverse velocity $u_{y}$ versus $y / w$ solved using Eqs. (4.16) and (4.18) with parameters selected to agree with MD simulation (see text).

To make a comparison between the theory and simulation, we need to determine an approximate value for $\alpha$ that represents the patterning of the surface. This was accomplished using the following method. From Fig. $4.10, \delta_{1} / w=0.36$ for $\varepsilon_{w f_{1}}=0.8 \varepsilon$ and $\delta_{2} / w=0.17$ for $\varepsilon_{w f_{2}}=0.9 \varepsilon$. The mean of these two is the average slip length $\delta_{0} / w=0.265$. Now the patterned-slip surface can be modelled by assuming a small fluctuation $\alpha$ about this mean value. Thus we define $\alpha$ using 
the following equations:

$$
\begin{aligned}
& \delta_{1}=\delta_{0}(1+\alpha) \\
& \delta_{2}=\delta_{0}(1-\alpha)
\end{aligned}
$$

Solving for $\alpha$ from the above two equations yields $\alpha=0.3463$. Although we could have used another set of interaction parameters to get a much smaller value of $\alpha$, we have observed that this leads to a very small transverse flow which is difficult to distinguish from thermal fluctuations. Thus we use the case $\alpha=0.3463$ to make a comparison between the continuum theory and MD simulations. The continuum equations were solved using the above set of parameters numerically and the result is shown in Fig. 4.15. Thus using Eqs. (4.16), (4.17) together with the value of $\alpha$ given above and the maximum magnitude from Fig. 4.15 we find a maximum transverse velocity $u_{y_{\max }}=0.0058 \sigma / \tau$ and this value is in good agreement with the MD result where $u_{y_{\max }}=0.007 \sigma / \tau$.

\subsection{Time Dependent Body Force}

We now make use of the viscoelastic property [119] of the polymer melts. Using continuum modelling, Pereira [1] showed that viscoelasticity of polymers can be utilized to enhance mixing further for these materials. In this study a time dependent sinusoidal pressure gradient was applied in addition to the patternedslip boundary conditions to enhance transverse flows. We carried out MD simulations to validate the predictions of this work. The time dependent pressure gradient was mimicked by applying a sinusoidal body force in the $x$ direction:

$$
F=F_{0}[1+\epsilon \sin (\omega t)]
$$

where the body force $F$ oscillates about the mean value $F_{0}$ with a small perturbation $\epsilon$ and $\omega$ is the frequency of the oscillation.

\subsubsection{Simulations of Homogeneous Channels with Sinusoidal Body Force}

Initially, we conducted MD simulations with a sinusoidal body force using homogeneous surfaces i.e., boundaries were not patterned. As we will see, this helps us in testing the elastic behaviour of the fluid. We apply a sinusoidal body force with a perturbation constant, $\epsilon$, in Eq. (4.25) set to 0.1 , so that the maximum value of this oscillating body force was $10 \%$ larger than for the constant case. The frequency $\omega$ was increased from $2 \pi / 100 \mathrm{rads} / \tau$ to $2 \pi / 10 \mathrm{rads} / \tau$. The body force 
was changed incrementally after every $\tau$ time to allow the thermostat to stabilize the temperature of the system. In each of these simulations the system was equilibrated roughly for $2 \times 10^{3} \tau$. The first thing we require to do is to verify that our fluid behaves like an elastic fluid. Thus when we apply a sinusoidal body force we expect an elastic fluid will also respond with a sinusoidal longitudinal velocity. However, because of viscous dissipation, the fluid will be out of phase with the body force. According to the continuum model of Pereira [1] the phase angle, $\phi$ that the longitudinal velocity lags behind the body force is related to the relaxation time $\Lambda$ and the forcing frequency, $\omega$ via

$$
\phi \equiv \arctan (\Lambda \omega)
$$

Simulations were carried out at frequencies $2 \pi / 100,2 \pi / 40,2 \pi / 30,2 \pi / 20$ and $2 \pi / 10 \mathrm{rads} / \tau$. Note, we cannot increase the frequency of the sinusoidal body force further since the polymer chains will not have sufficient time to respond to changes in body force.

In response to the sinusoidally varying body force, we expect a sinusoidally varying Poiseuille flow in the channel. In each frequency case, by monitoring the maximum of the Poiseuille velocity profile $\left(u_{x_{\max }}\right)$ in the centre of the channel, we found that the response of the fluid was indeed sinusoidally time varying and this confirms that the fluid is indeed elastic. To demonstrate this effect we present the results of the body force and corresponding longitudinal velocity response in Fig. 4.16 for the case where $\omega=2 \pi / 100 \mathrm{rads} / \tau$. The phase lag $\phi$ between the longitudinal force and the velocity further affirms the elasticity of the fluid. Note, the longitudinal velocity shown was an average over 1000 configurations, where the averaging was done every $T \tau$, where time period $T=10,20,30,40,100$ depending on the particular frequency being studied. The phase lag is marked by the blue line in Fig. 4.16. To obtain a precise value of this phase lag we fit the longitudinal velocity data (circles) to a sinusoid, $A \sin (\omega t+\phi)+A_{0} \cdot A_{0}$ and $A$ can be obtained from the data while $\omega$ is known. We carry out a least squares fit to obtain $\phi$. In each case the least squares error was found to be less than $10^{-3} \mathrm{rad}$. The phase lags calculated according to this method are given in Table 4.1. As the forcing frequency increases we find the phase lag correspondingly increases, which is expected since for higher frequencies the polymer chains cannot respond sufficiently quickly, leading to a larger phase lag. The largest phase lag that can occur is $\pi / 2 \mathrm{rad}$. Now, according to Eq. (4.26) we can also obtain $\Lambda$ for the polymer melt. These are also tabulated in Table 4.1. Interestingly, for each forcing frequency we find a different $\Lambda$, with the $\Lambda$ s systematically increasing 


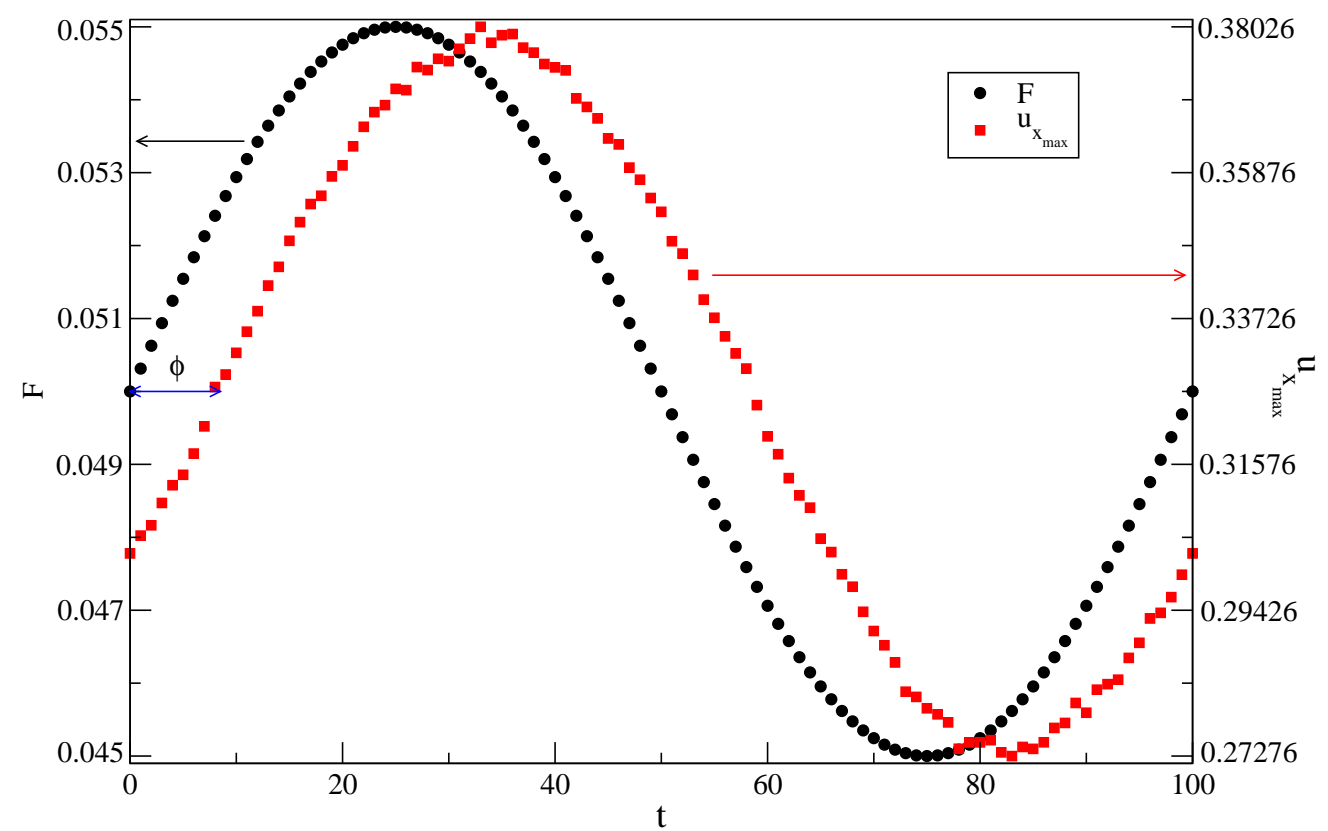

Figure 4.16: The body force (circles) and maximum longitudinal velocity response (squares) as a function of time. The scale on the left-hand side represents body force and the one on the right-hand side represents the magnitude of $u_{x_{\max }}$.

\begin{tabular}{|c|c|c|c|c|c|}
\hline$\omega$ & $2 \pi / 100$ & $2 \pi / 40$ & $2 \pi / 30$ & $2 \pi / 20$ & $2 \pi / 10$ \\
\hline$\phi$ & 0.516 & 1.007 & 1.167 & 1.375 & 1.571 \\
\hline$\Lambda$ & 9.02 & 10.08 & 11.17 & 16.08 & $\infty$ \\
\hline$u_{y_{\max }}$ & 0.0287 & 0.0284 & 0.0283 & 0.0281 & 0.0288 \\
\hline$\%$ increase & 21.2 & 20.1 & 19.7 & 18.8 & 21.7 \\
\hline
\end{tabular}

Table 4.1: Phase lag $(\phi)$, relaxation time $(\Lambda)$, maximum of transverse velocity $\left(u_{y_{\max }}\right)$ and percentage increase in maximum transverse velocity compared to constant body force case for various sinusoidally varying body force frequencies.

with frequency.

\subsubsection{Simulations of Patterned Channels with Sinusoidal Body Force}

In the continuum model of Pereira [1], the viscoelastic behaviour of the fluid was modelled using a linearized White-Metzner model [120]. According to this model

$$
\underline{\underline{\tau}}+\Lambda \frac{\partial \underline{\underline{\tau}}}{\partial t}=-\eta(\dot{\gamma}) \underline{\underline{\dot{\gamma}}},
$$

where $\Lambda$ is the relaxation time of the fluid. The longitudinal and transverse velocity profiles can be obtained by using the perturbative approach by expanding 
the velocity profiles in terms of $\alpha$ and $\epsilon$.

$$
\begin{aligned}
& u=u_{0}+\alpha u_{1}+\epsilon\left(u_{10}+u_{11} \sin (\omega t)\right)+\alpha \epsilon\left(u_{\alpha 10}+u_{\alpha 11} \sin (\omega t)\right)+O\left(\alpha^{2}, \epsilon^{2}\right)+\cdots \\
& v=\alpha v_{1}+\alpha \epsilon\left(v_{\alpha 10}+v_{\alpha 11} \sin (\omega t)\right)+\cdots
\end{aligned}
$$

The sinusoidal body force effect on the transverse flow can be quantified by solving $v_{\alpha 11}$ [1]. Solving the Navier-Stokes equations using the above set of equations with appropriate boundary conditions yields

$$
\left|v_{\alpha 11}\right| \propto\left(1+\Lambda^{2} \omega^{2}\right)^{1 / 2}
$$

Hence the model predicts that the transverse velocity is proportional to the frequency of the sinusoidal body force.

As we saw from our simulations, the fluid has more than one relaxation time. Thus we see an important deficiency of the simplified continuum model [1] - it employs only a single relaxation constant, $\Lambda$. As pointed out above this simplification was used to obtain tractable solutions. However, a better model for our viscoelastic fluid would be a Generalized linear viscoelastic model [56], that is a superposition of linear White-Metzner models of the form

$$
\underline{\underline{\tau}}(t)=\sum_{k=1}^{\infty} \underline{\underline{\tau}}_{k}(t) ; \quad \underline{\underline{\tau}}_{k}+\Lambda_{k} \frac{\partial \underline{\underline{\tau}}_{k}}{\partial t}=-\eta_{k}(\dot{\dot{\gamma}}) \underline{\underline{\dot{\gamma}}} .
$$

Given that there is not a single $\Lambda$ that describes our fluid, but in principle there is an infinite number of them we might not expect the response of the fluid to be exactly as specified by Eq. (4.30).

\subsubsection{Comparison between Constant Body Force and Sinusoidal Body Force Simulations}

We now proceed to discuss the simulations with the (same) patterned boundary conditions as discussed in the earlier section but in conjunction with the sinusoidal body force. Now the transverse velocity profile changes with the body force which is a sinusoidal function of time. To calculate average transverse velocity profiles we have to consider only those profiles which are separated by $T \tau$. We take 5000 such configurations to evaluate the transverse velocity profiles for each frequency which was found to be a sufficient number of averages 
to compare with the constant body force case, as averages obtained using more configurations than this did not show any significant change. Note, to carry out these 5000 averages for the simulation with forcing frequency of $2 \pi / 100 \mathrm{rads} / \tau$ took approximately 7 days of cpu time on a parallel Blue-Gene machine with 128 processors. At the moment, this is the smallest frequency we can simulate so as to obtain reliable results.

As with the simulations for homogeneous slip boundary conditions, we carried out simulations at frequencies of $2 \pi / 100,2 \pi / 40,2 \pi / 30,2 \pi / 20$, and $2 \pi / 10 \mathrm{rads} / \tau$ keeping the pattern length constant at $k w=\pi / 2$. The resulting transverse velocity profile for the case $\omega=2 \pi / 10 \mathrm{rads} / \tau$ is shown in Fig. 4.17. Since we have taken these averages after every $T \tau$ time the velocity profile has

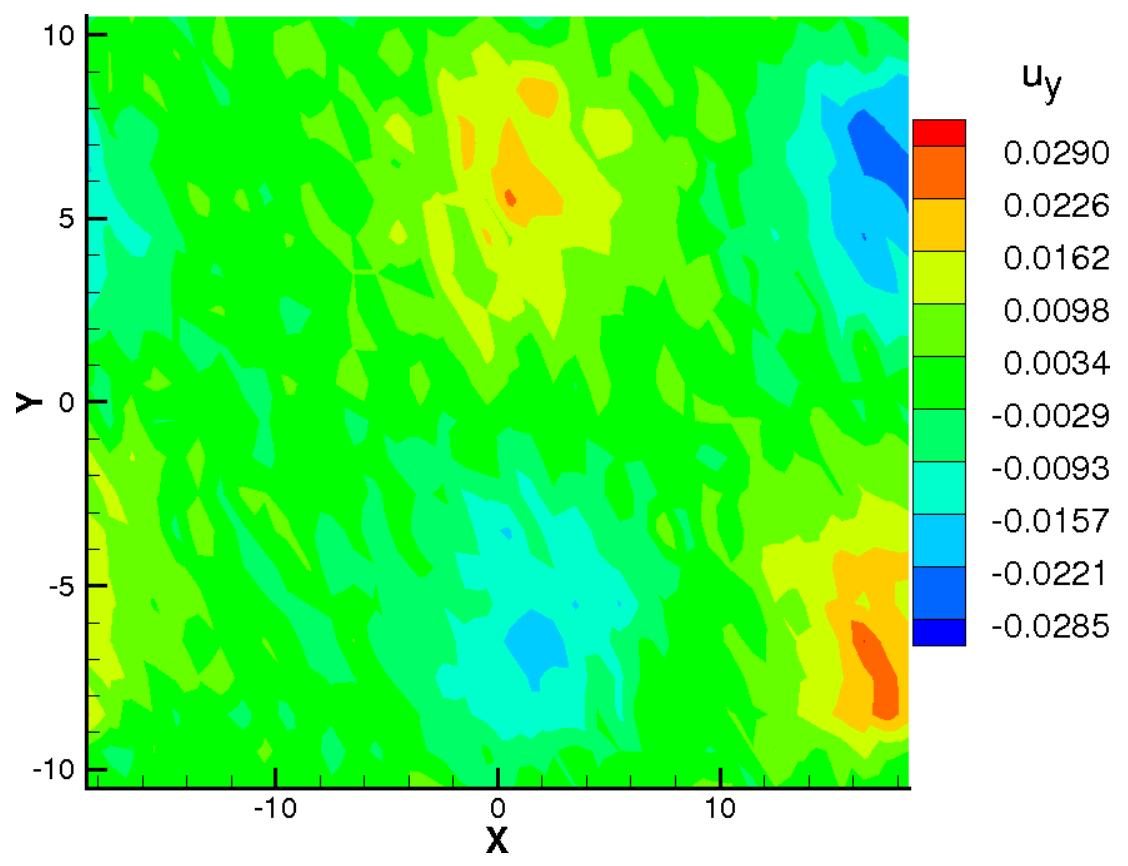

Figure 4.17: Transverse velocity profile $u_{y}(x, y)$ for the time dependent body force case for the frequency $\omega=2 \pi / 10$.

a similar form to the constant body force case. To compare the magnitude of the transverse velocity with the constant body force case we have calculated the transverse velocity profile for the same setup with the constant body force $\left(F_{0}=0.05 m \sigma / \tau^{2}\right)$ by taking 5000 averages (See Fig. 4.18).

The transverse velocity profile in Fig. 4.17 is shifted compared to the constant body force case in Fig. 4.18. This result also complements the viscoelasticity of the fluid. With oscillatory body force, the maximum value of the transverse velocity has increased to $0.0288 \mathrm{~m} \sigma / \tau^{2}$ (compared to a maximum value of $0.0236 m \sigma / \tau^{2}$ in the constant body force case). This represents a $22 \%$ increase 


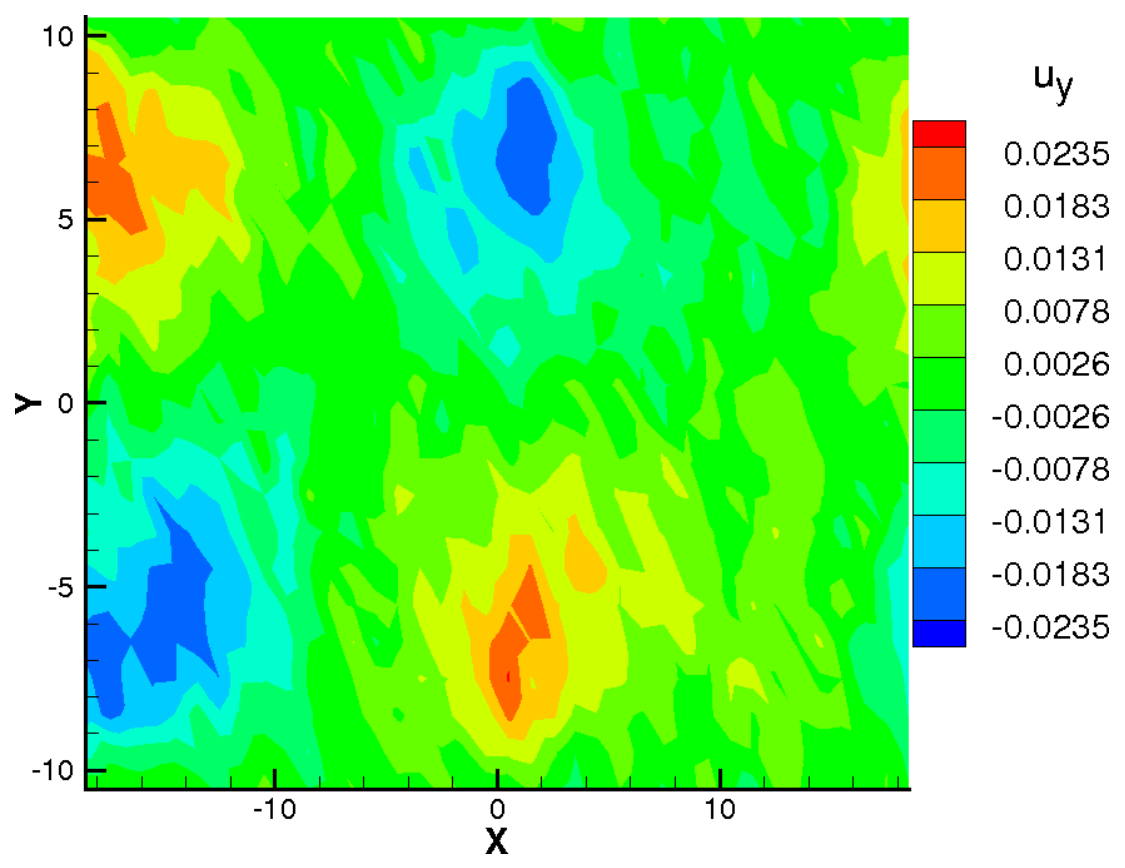

Figure 4.18: Transverse velocity profile $u_{y}(x, y)$ for constant body force case with 5000 averages.

in the transverse velocity. Results of simulations for other values of $\omega$ are given in Table. 4.1. In general, we find the increase is on average $20 \%$, compared to a constant body force, and given we have applied a maximum $10 \%$ increase in the body force this represents a reasonable increase. We see that the maximum transverse velocity does not change appreciably with increasing frequency. Thus although our simulations agree with the continuum theory in that a sinusoidally varying body force will increase the transverse velocity, the simulations do not show transverse velocities which increase as $\omega$ increases. We believe the reason for this is that the simplified continuum model includes only a single $\Lambda$ while the fluid modelled is characterized by several relaxation constants. Instead the transverse velocity be a function of a combination of velocities corresponding each relaxation constant. Hence Eq. (4.30) does not hold.

Another deficiency of the continuum model [1] is that it is only a linear viscoelastic model. When compared to our MD simulations this is not such a serious problem, since we use perturbations, $\epsilon$ which are quite small (i.e, 10\% of mean body force). However, certainly for larger perturbations a non-linear model would be more appropriate. 


\subsection{Summary}

In this study we have sought to model the flow of polymeric fluids in narrow channels at a microscopic level. We have focused on enhancing the mixing in these fluids with a combination of active and passive techniques. The passive method is to pattern the channel boundaries with regions of high and low slip while the active method is to employ a sinusoidally varying pressure. We first modelled the passive method by varying the interaction between monomers and wall atoms. The polymeric fluid was characterized as a shear-thinning fluid and we found significant transverse flows, especially when the pattern spacing was greater than the radius of gyration of a polymer chain. In this case polymers tend to stick or slip adjacent to the different patterned regions, thus perturbing the dominant Poiseuille flow. In the case that the pattern spacing is smaller than the radius of gyration of the polymer chains, the transverse velocity is suppressed, as the polymer chains span two or more regions and begin to interact with them as if the boundaries were homogeneous. Thus the flow returns to being strongly Poiseuille-like. In general we found qualitative agreement with previous continuum theory [1]. We made quantitative comparison with this theory and have found good agreement on the magnitude of the generated transverse velocity. We proceeded to consider the effect of an off-set arrangement of the patterned regions. In this case, although the magnitude of the transverse velocity did not increase, it was an improvement on the previous case, since now transverse flow is generated throughout the width of the channel (see Fig. 4.14). In the case where the patterned regions are aligned on either side of the channel, transverse flow is zero in the middle of the channel for the entire length of the channel. Hence, fluid in this region will not mix by convection (only via diffusion). We have continued on to couple a sinusoidally varying pressure gradient with the patterned boundary conditions. In this case the continuum theory had predicted an enhancement of the transverse velocity. Our simulations also found an enhancement of the transverse flow for a sinusoidally varying pressure. The increase $-20 \%$ results from a 10\% increase in the amplitude of the pressure. The continuum modelling also predicted that this transverse velocity should increase with frequency. However, our simulations did not show this for two reasons: (i) the polymeric fluid was found to have a number of relaxation constants (whereas the continuum theory assumes only one) and (ii) there is an upper bound on the magnitude of frequency, since polymer chains cannot respond sufficiently quickly for large frequencies. 


\section{Chapter 5}

\section{Capillary \\ Absorption of Polymer Droplets}

When a capillary (narrow hollow tube) is lowered into a liquid, the liquid either ascends or descends the capillary. This phenomenon is known as capillary action or capillarity and it has a wide range of applications both in biological and industrial processes $[35,32]$. In general, the standard continuum models on capillarity [27, 28, 29, 30] describe this phenomena very well. However, these models make certain assumptions that may not be always valid [35]. In particular, here we shall focus on one particular assumption: size of the liquid reservoir. Standard macroscopic theories on capillarity assume that the liquid reservoir is infinite (compared to tube dimensions). However, this is not always the case. For example, industrial applications such as ink-jet printing, spray painting, etc. deal with finite liquids [35, 36]. Marmur [35] first studied the effect of finite size of the liquids on the statics and dynamics of capillarity. He found that the Laplace pressure originating from the curvature of the droplet (due to finite size of the liquid) plays an important role in determining the underlying capillary process. He showed that capillary absorption of sufficiently small, non-wetting droplets is possible. This is in contrast with the standard macroscopic models where capillary uptake of non-wetting liquids is prohibited. As we scale down, these finite size effects become more profound and this can give rise to novel phenomena which are otherwise absent. For example, using Marmur's arguments, Schebarchov and Hendy [37] were able to explain the absorption of non-wetting metal droplets into carbon nanotubes (CNTs) [121]. Macroscopic models on capillarity cannot explain such phenomena.

There is a growing interest in building polymer-based functional nanomate- 
rials for novel applications [122, 123, 124, 125, 126, 127, 128]. One of the methods used for producing such structures is by filling nanomoulds using capillary forces. For example, polymer-based nanopattern wires [129], nanorods and nanotubes [40], nanoscale protein patterning [130], nanofibers [131], nanobelts [132], etc. were produced using capillary action. Most of the methods involved in the production of such materials fall in the above discussed regime, viz the size of the liquid reservoir is finite compared to the channel dimensions. Thus, the Laplace pressure driven capillary forces can become important. Nanoscale phenomena of polymer solutions or melts is particularly fascinating because at these dimensions, their characteristic length scales become comparable with those of device dimensions. This can have important implications on the behaviour of polymers in these devices, which can lead to new phenomena $[7,38]$.

Moreover, the capillary dynamics of polymers can be important in the above mentioned applications, involved with producing polymer-based nanomaterials. In the context of nanocapillaries, the question that received most attention, recently, is whether the Lucas-Washburn law [29, 30] of capillary rise applies at the nanoscale [31]. This is yet to be fully solved, with the literature full of conflicting results $[31,32,33,34,133,134,135,136]$. At the nanoscale, as mentioned earlier, confinement effects on polymers become prominent and this can impact the underlying capillary dynamics in a major way.

In this chapter, we study the capillary phenomena of small polymer droplets in narrow tubes using MD and theoretical analysis. In particular, we focus on the macromolecular aspect of polymers and study its effect on both statics and dynamics of capillarity. The chapter is organized as following. In section 1 we introduce the basics of capillarity and discuss the role of finite size effects. In section 2 we carry out the characterization of the droplet and in section 3 we present the contact angle simulations. In section 4 we present the results and discussion of the capillarity of polymer droplets. In section 5, a theoretical analysis of capillary phenomena of polymer droplets is carried out and the results are compared with the simulations. Finally, in section 6 we conclude the findings from this study.

\subsection{Basics of Capillarity}

The fundamentals of capillarity were established in the early part of 19th century by Young [27] and Laplace [28]. Their theory forms the basis for much of our understanding of the static equilibrium conditions for capillarity. The physical foundation for capillarity lies in understanding the concept of surface tension, which is a quantitative measure of the surface properties at the macro- 
scopic level. Molecular interactions at the microscale give rise to this property. To understand this statement, consider a molecule in the interior of a liquid bulk, it experiences cohesive forces from surrounding molecules in all directions. Hence, the net resultant force acting on a molecule within the bulk is nearly zero. However, this is not the case for the molecules that form the surface. Due to loss of neighbours on one side, these molecules experience a net inward force, perpendicular to the surface and into the bulk; and this gives rise to surface tension. Quantitatively, it is defined as the amount of energy required to increase the surface by unit area. If a system contains more than one type of molecule, then there exist adhesive forces between dissimilar molecules in addition to cohesive forces between similar molecules. When there is more than one type of molecule the term 'surface tension' is replaced by 'interfacial tension'. However, in this chapter we shall use both of these words interchangeably.

The relative competition between the cohesive and adhesive forces determines the nature of the liquid-vapour interface. Based on thermodynamic arguments, Young [27] derived the condition for static equilibrium for a liquid-solid system (see Fig. 5.1) in terms of interfacial tensions:

$$
\gamma_{S V}=\gamma_{S L}+\gamma_{L V} \cos \theta_{C}
$$

where the subscripts $S, L$ and $V$ denote solid, liquid and vapour phases; $\gamma_{S V}, \gamma_{S L}$ and $\gamma_{L V}$ are the interfacial tensions associated with the solid-vapour, solid-liquid and liquid-vapour surfaces. $\theta_{c}$ is referred to as the contact angle between the liquid-vapour and solid-vapour interfaces. If $\theta_{c}<90^{\circ}$, the liquid is said to partially wet the surface with the limiting case $\theta_{c}=0^{\circ}$, total wetting; and if $\theta_{c}>90^{\circ}$ then the liquid is said to be non-wetting.

Due to surface tension liquids try to minimize their surface area. Acting against this is the pressure difference across the liquid-vapour interface. A delicate balance between these two competing forces determines the equilibrium shape of the liquid surface. In the case of a spherical droplet, the pressure difference across the surface is given by

$$
\Delta P=\frac{2 \gamma_{L V}}{R}
$$

Here $\gamma_{L V}$ is the liquid-vapour surface tension and $R$ is the radius of the droplet. From here on we drop the subscript $L V$ in $\gamma_{L V}$, for simplicity.

When a capillary is immersed in a liquid, the liquid ascends or descends 


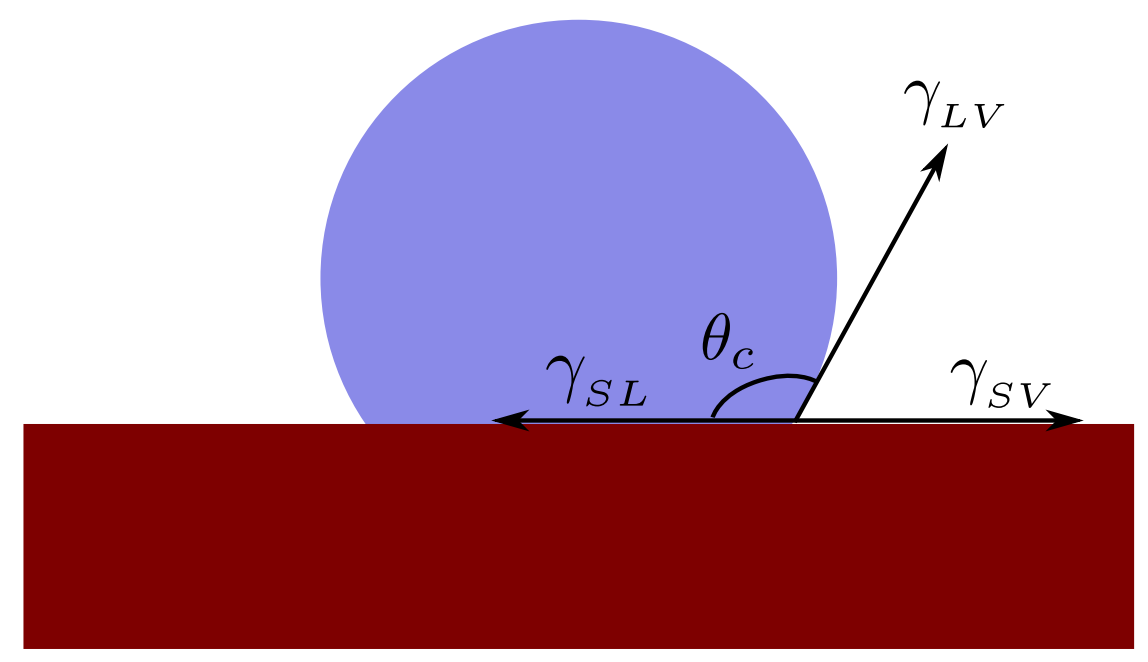

Figure 5.1: An equilibrium configuration of a droplet-substrate system. The locus of points where the solid-liquid interface meets the liquid-vapour interface is known as the contact line. The angle between these two interfaces at the contact line is the contact angle $\left(\theta_{c}\right)$ of the droplet with respect to the solid.

the capillary depending on its wetting behaviour. If the liquid likes to wet the surface then adhesive forces are stronger than cohesive forces and it rises up the tube; and vice-versa. For a capillary immersed in an infinite liquid reservoir, the equilibrium height of the liquid column inside the tube, $H$, can be determined by equating the pressure difference across the meniscus due to surface tension to the gravitional force:

$$
H=\frac{2 \gamma \cos \theta_{c}}{\Delta \rho g R_{t}}
$$

where $\Delta \rho$ is the difference in densities between liquid and vapour phases, $g$ is the acceleration due to gravity and $R_{t}$ is the radius of the tube. Note that $H$ is positive for $\theta_{c}<90^{\circ}$ i.e., liquid ascends the tube whereas for $\theta_{c}>90^{\circ}, H$ is negative i.e., liquid descends below the height of the liquid reservoir.

The dynamics of capillarity were first studied by Lucas [29] and Washburn [30] independently. They derived the equation for the height of the liquid column as a function of time assuming (i) that the flow has reached quasi-steady state conditions, (ii) the liquid reservoir to be infinite, (iii) neglecting the inertial and frictional effects, (iv) that the tube radius is constant, and (v) the contact angle is constant. The Lucas-Washburn equation is given by

$$
H(t)=\left(\frac{\gamma R_{t} \cos \theta_{c}}{2 \eta}\right)^{1 / 2} \sqrt{t}
$$

where $\eta$ is the viscosity of the fluid. An important point to note is the relationship $H(t) \propto \sqrt{t}$, as we will come back to this later in the Chapter. From Eqs. (5.3) and 
(5.4), capillary uptake of liquids is not possible for $\theta_{c}>90^{\circ}$.

The capillary models discussed above assume that the liquid reservoir is infinite. However, as pointed our earlier, this is not always true. Here we briefly discuss Marmur's work in this regard due to its relevance to our study.

Consider a droplet and tube setup as shown in Fig. 5.2. Compared to the case of an infinite reservoir here we have two liquid surfaces. The additional surface from the protruding droplet results in a Laplace pressure which was absent in the infinite liquid reservoir case. The static equilibrium condition for such a system, in the absence of gravity is described by [35]

$$
\frac{2 \gamma}{R_{d_{e}}}+\frac{2 \gamma \cos \theta_{c}}{R_{t}}=0
$$

where $R_{d_{e}}$ and $R_{t}$ are the radii of the protruding droplet at equilibrium and the tube, respectively. The first term is due to the Laplace pressure and the second term is due to the pressure difference across the meniscus.

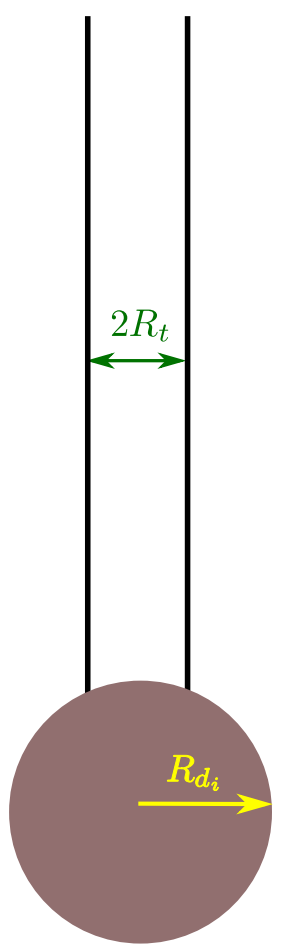

(a) Initial configuration
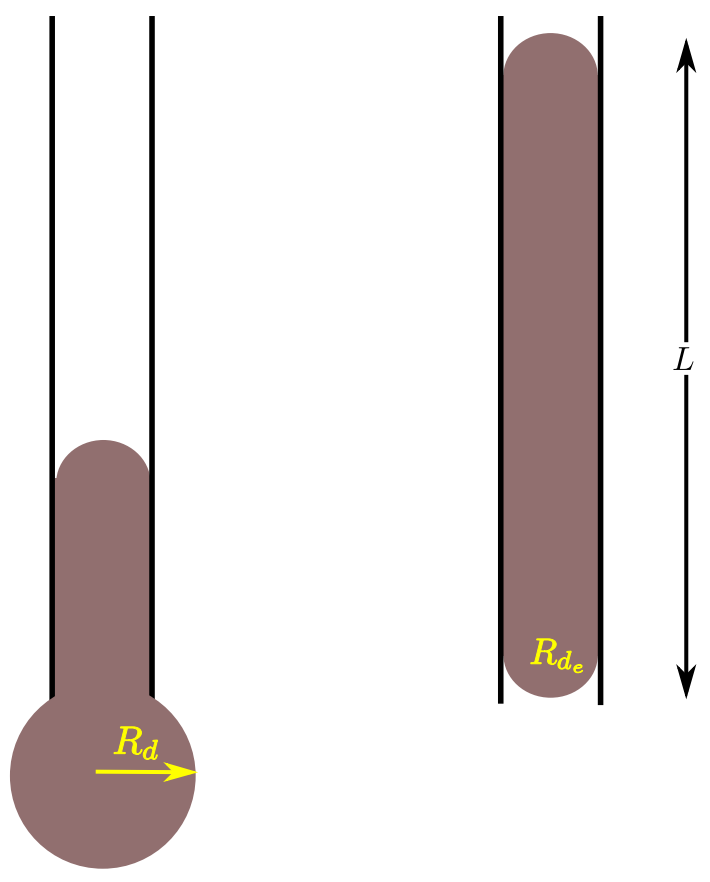

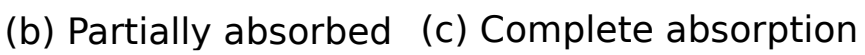

Figure 5.2: (a) The initial system setup, $R_{d_{i}}$ is the initial radius of the droplet and $R_{t}$ is the radius of the tube. (b) An intermediate stage during the absorption process with $R_{d}$ being the radius of the protruding droplet. (c) The droplet gets totally absorbed into the tube and $R_{d_{e}}$ is the final equilibrium radius of the protruding droplet. 
Now let us try to obtain the condition for capillary absorption in terms of contact angle. At equilibrium Eq. (5.5) takes the following form

$$
\frac{R_{d_{e}}}{R_{t}}=-\frac{1}{\cos \theta_{c}}
$$

From Eq. (5.6) it can be inferred, for $\theta_{c}<90^{\circ}, R_{d_{e}}$ is negative, and the droplet will be absorbed for all radii $R_{d_{i}}>0$. When the liquid is non-wetting, the contact angle $\theta_{c}>90^{\circ}$ and $R_{d_{e}}>0$, liquid will ascend the tube as long as the radius of the protruding droplet $R_{d}$ is greater than a certain critical radius $R_{d_{e}}$ (equilibrium radius of the protruding droplet) even for contact angles greater than $90^{\circ}$ ! This result adds a new dimension to the classical theories which do not permit capillary action for non-wetting fluids. Note that from Eq. (5.6), the equilibrium droplet radius below which absorption takes place also depends on the radius of the tube. For smaller tubes the droplet size has to be smaller to be able to observe absorption.

Unlike their Newtonian counterparts, polymeric fluids possess conformational entropy, originating from their macromolecular structure. In the case of capillary absorption of polymers in tubes comparable to chain size, if a droplet were to ascend the tube then its constituent chains must stretch in the direction of tube axis. This will result in loss of conformational entropy. Thus, in the case of polymer droplets, in addition to the Laplace pressure, which pushes the droplet into the tube, we have the conformational entropy loss which opposes the droplet motion into the tube. The trade-off between these two competing forces (assuming that the meniscus pressure is neutral) should determine whether the droplet gets absorbed or not. Hence, the aim of this study is to examine the effect of molecular size on the entire process of capillary driven absorption. This is realized by studying the capillary phenomena in droplets of the same size (total number of monomers is constant) while varying the chain length of the constituting chains.

\subsection{Characterization of The Droplet}

The initial configurations for polymer droplets were prepared by randomly placing the constituent chains in a spherical volume to realize the system density at $0.85 \sigma^{-3}$. Since the initial configuration consisted of highly overlapping chains we used the soft potential method discussed in Chapter 4 to bring the system to equilibrium. The temperature of the system was kept constant at $T=1.0 k_{B} T / \varepsilon$ by coupling the monomers with the Langevin thermostat with a 
friction coefficient of $\Gamma=1.0 \tau^{-1}$. The equations of motion were integrated using the velocity Verlet algorithm.

During the equilibration period, for the first $6 \tau$ the interaction between monomers was described by the soft potential with $r_{c u t}=2^{1 / 6} \sigma$ and the equations of motion were integrated with $\Delta t=0.0001 \tau$. After this time, the potential energy remained approximately constant and this confirms that the system has reached equilibrium. At this point, interaction between the monomers was switched from the soft potential to the LJ potential with parameters $\varepsilon=1.0$ and $\sigma=1.0$ and the timestep size was changed to $\Delta t=0.01 \tau$.

The average properties of chains were extracted by noting down droplet configurations every $100 \tau$ for the next $5 \times 10^{4} \tau$. This method was applied to droplets made up of different chain lengths but having the same number of total monomers. (For example for a droplet of 8000 monomers we ran separate simulations of 400 chains of length 20, 80 chains of length 100 and so on.) The average radius of droplets with 8000 monomers was approximately $\left\langle R_{0}\right\rangle \approx 15.03 \pm 0.07 \sigma$, where the error estimate is the standard deviation computed over droplets of all chain lengths studied. As we can see there is very little effect of chain length on the droplet size. For droplets with 16000 monomers, $\left\langle R_{0}\right\rangle \approx 18.58 \pm 0.07 \sigma$.

We now compute the average chain size $\left\langle R_{g}\right\rangle$, averaged over all the chains in the droplet, for droplets of different chain lengths (at constant total number of monomers). The time average of this quantity was obtained by computing an average over 500 droplet configurations over the simulation period and the results are shown in Fig. 5.3 for 8000 monomer droplets. In the small $N$ limit, on average the chains behave as if they were ideal chains, $\left\langle R_{g}\right\rangle \propto N^{0.5}$. There appears to be cross-over from this behaviour for chain lengths $N>200$. A possible explanation for this trend may be as follows. For small $N$, each chain on average is surrounded by a large number of chains, equally in all directions. This is analogous to a chain in a melt which is ideal. As $N$ increases, each chain on average will have fewer polymer neighbours, i.e. more exposure to solvent. Therefore, the trend in $\left\langle R_{g}\right\rangle$ deviates from the ideal chain behaviour.

We also compared these results with single chain simulations, conducted under the same conditions as droplet simulations. The $\left\langle R_{g}\right\rangle$ behaviour from these simulations for different chain lengths is plotted in Fig. 5.3. The single chain simulations reveal that the chains display poor solvent behaviour, where $\left\langle R_{g}\right\rangle \propto N^{0.33}$. The $\left\langle R_{g}\right\rangle$ from the droplet simulations, as $N$ approaches the system size ( $N \rightarrow$ total number of monomers in the droplet), converges onto the single 


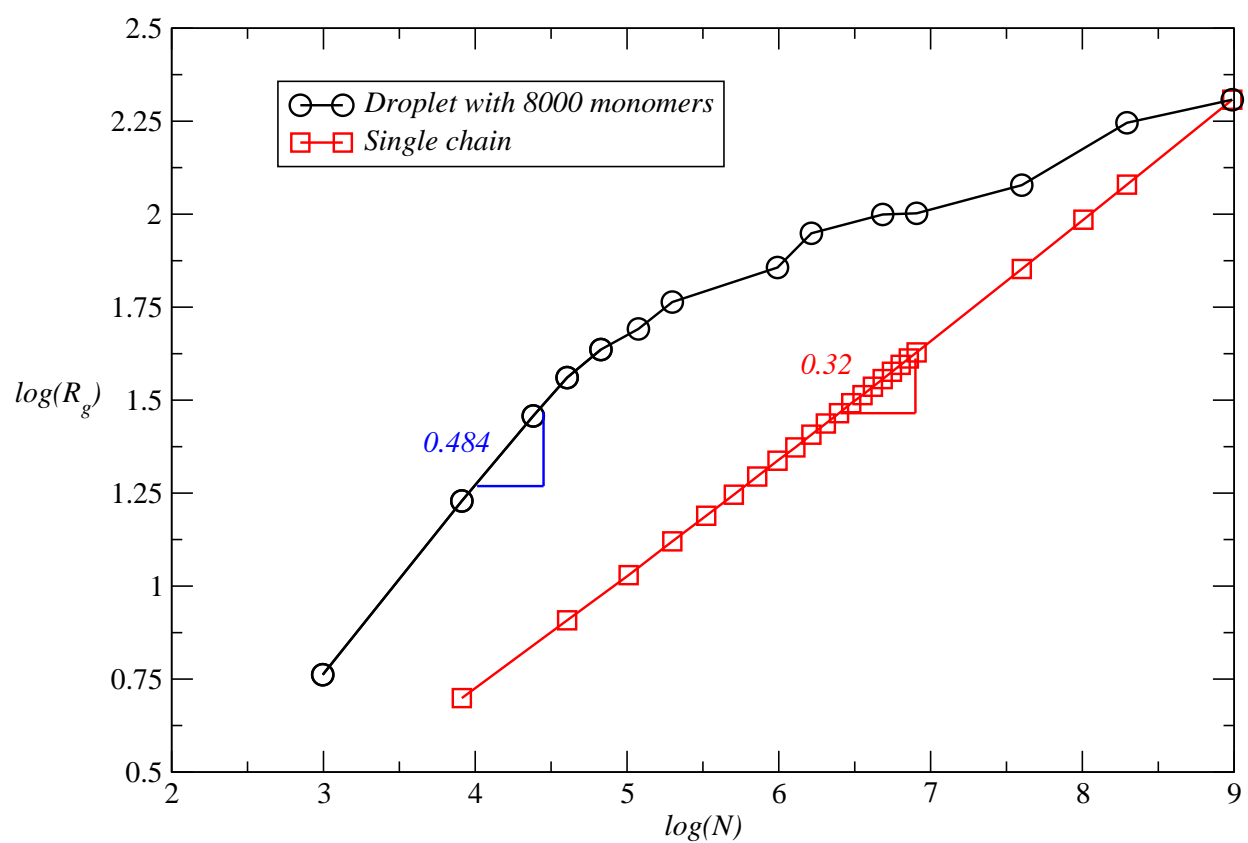

Figure 5.3: The $\left\langle R_{g}\right\rangle$ behaviour as a function of chain length. The black curve (circles) represents the average size of a chain in the droplets of 8000 monomers. The red curve (squares) is data from single chain simulations.

chain results. This trend supports our earlier explanation for the shrinkage in $\left\langle R_{g}\right\rangle$, for droplets with larger $N$ values. Simulations for larger droplets with 16000 monomers revealed that $\left\langle R_{g}\right\rangle$ behaviour was similar to 8000 monomer droplet except for a slight deviation in the large $N$ limit. This is because on average each chain has more neighbour chains in a 16000 monomer droplet than in 8000 monomer droplet.

\subsection{Contact Angle Simulations}

The nature of the solid-liquid-vapour interface is related to the contact angle. The contact angle can be used to discriminate between wetting and non-wetting surfaces. As discussed in the earlier sections of this Chapter the value of contact angle is essential for studying capillary action. Though the concept of contact angle becomes ambiguous for very small systems, we shall nevertheless attempt to capture the approximate behaviour of the liquid-vapour interface. It is well known that the surface tension of polymeric fluids depends on the molecular weight of the comprising chains [137, 138], which will in turn affect the contact angle. We test the molecular size dependence of the contact angle in our simulations. Within the framework of MD simulations, control over the contact angle can be gained by tuning the interaction between the liquid and solid constituents. By varying this interaction the wetting properties of the 


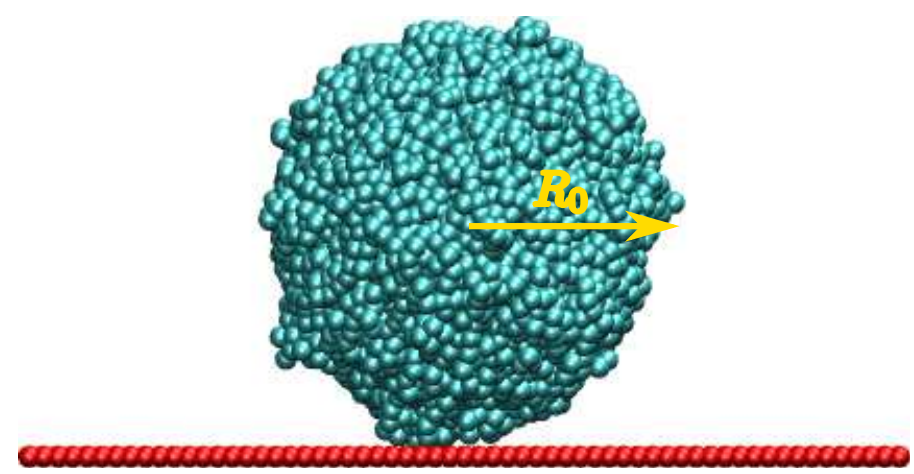

(a) Initial configuration

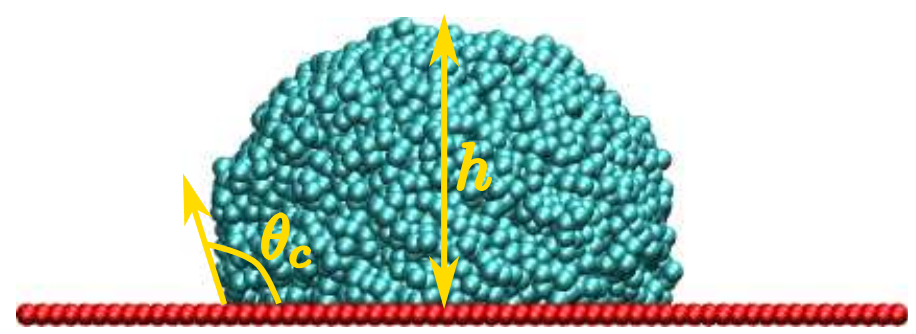

(b) Equilibrated configuration

Figure 5.4: Initially the droplet of radius $R_{0}$ was placed on the substrate. After equilibration the droplet assumes the shape of an equilibrium spherical cap of height $h$ making an angle $\theta_{c}$ with the substrate.

liquid can be changed. A weak interaction results in a large contact angle due to domination of cohesive forces over adhesive ones and vice-versa.

Equilibrated liquid droplets were placed on a single atom thick simple cubic substrate with lattice constant $a=1.0 \sigma$. The substrate atoms were fixed in space to reduce the computational effort. The interaction between the substrate atoms and monomers was given by the LJ potential with $\sigma=1.0$. In general, for wetting liquids, a precursor foot is found to spread ahead of the bulk droplet and hence the contact angle becomes time-dependent [139, 140]. Therefore, to avoid ambiguity in determining the contact angle we restrict ourselves to non-wetting droplets. A LJ interaction strength of $\varepsilon_{p s}=0.5 \varepsilon$ fulfills this condition $\left(\theta_{c}>90^{\circ}\right)$ in our simulations. The temperature of the droplet was maintained constant by coupling the Langevin thermostat to all the monomers with a friction coefficient of $\Gamma=1.0 \tau^{-1}$. Though the Langevin thermostat screens the hydrodynamic effects by its very virtue, this does not cause serious concern as we are only interested in the equilibrium properties. The equations of motion were integrated using the velocity Verlet algorithm with a timestep $\Delta t=0.01 \tau$.

The droplet was initially equilibrated for $10^{4} \tau$ on the substrate to let it attain its equilibrium height. After this point, we note down the configurations every 
$100 \tau$ for the next $4 \times 10^{4} \tau$ to calculate averages. The equilibrium contact angles were extracted by approximating the equilibrated droplet to a spherical cap. If a droplet has an initial radius $R_{0}$, and assumes a spherical cap geometry of height $h$, after equilibration, then the contact angle formed by the spherical cap is

$$
\theta_{c}=\cos ^{-1}\left(1-\frac{3}{4 R_{0}^{3} / h^{3}+1}\right)
$$

The initial and final configurations from one of the simulations is shown in Fig. 5.4. From our simulations we calculate time averages of the quantities $R_{0}$ and $h$, over the averaging period. These quantities are then substituted into Eq. (5.7) to extract an approximate contact angle, for that particular case. These measurements were repeated for different chain lengths and droplet sizes, to quantify their effect on the contact angle. The results are shown in Fig. 5.5. The

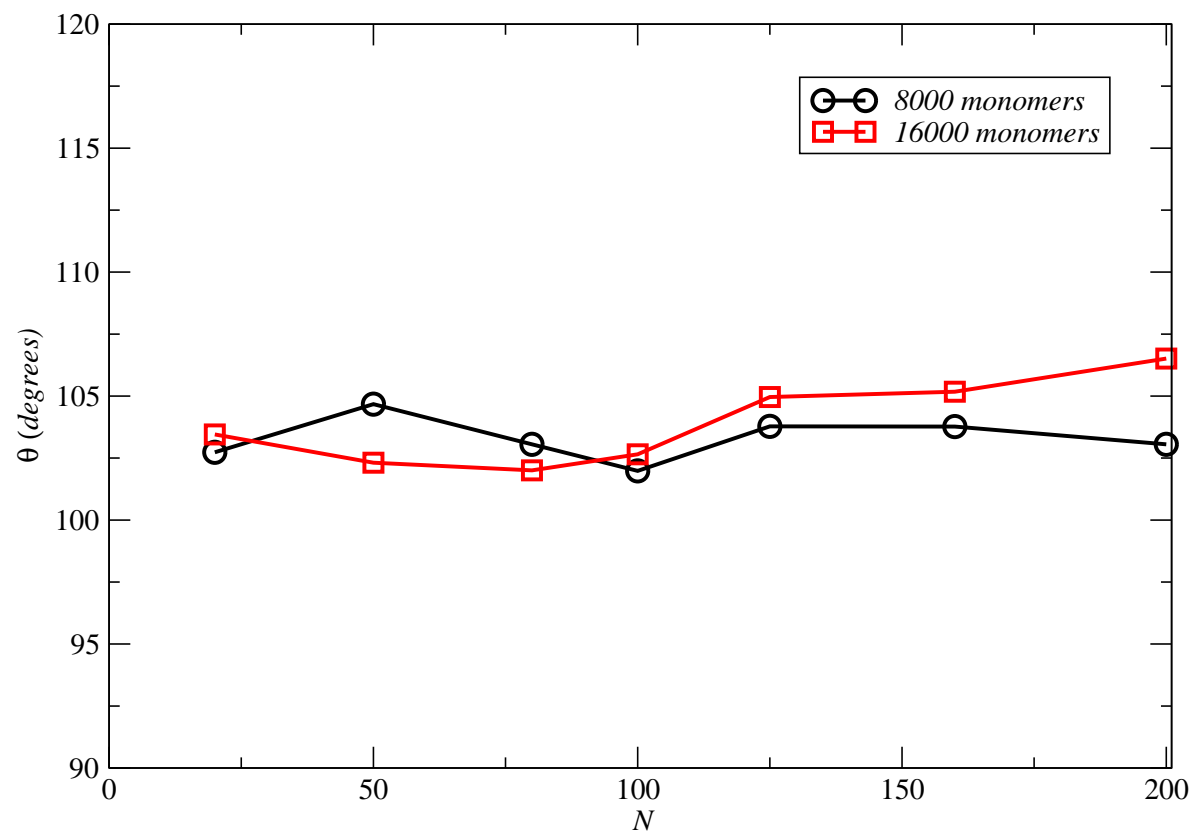

Figure 5.5: Contact angle measurements as a function of chain length, $N$ are plotted here. The black curve (circles) represents the data from 8000 monomer droplets and the red curve (squares) represents the data from 16000 monomer droplets.

contact angle remains approximately constant irrespective of the chain length and the droplet size, within respective parameter ranges shown in Fig. 5.5. These results are in good qualitative agreement with earlier observations [141, 142]. We estimate the average contact angle for the case of $\varepsilon_{p s}=0.5 \varepsilon$ as $\theta_{c} \approx 104^{\circ}$ with in $1 \%$ error. 


\subsection{Capillary Absorption of Polymer Droplets}

In the context of non-wetting polymer droplets, for capillary absorption to take place, the Laplace pressure must overcome conformational entropy loss in addition to meniscus pressure. It is also worthwhile to consider the dynamics of the underlying process. As mentioned earlier, the validity of the Lucas-Washburn equation at the nanoscale is debatable. We suspect entanglement effects will play a role in determining the dynamics at nanoscale. Entangled polymers are known to display different dynamic regimes depending on their chain lengths. Melts with shorter chains exhibit Rouse-type dynamics whereas those composed of longer chains showcase reptation-type dynamics [51, 143]. Therefore, it is of considerable interest to study the effect of molecular size on the statics and dynamics of polymer droplets under capillary action. For this purpose, we carry out MD simulations of polymer droplets of the same size (total number of monomers is constant) but composed of different chain lengths. We restrict our simulations to two cases, $N=20$ and 200 as the former falls in the Rouse regime and the latter falls in the reptation region [143]. This difference in chain dynamics should capture any significant differences in the observed capillary absorption dynamics.

The tube was constructed by rolling a single atom thick, simple cubic layer with a lattice constant $a=1.0 \sigma$ into a cylinder with both ends of the tube being open. The tube atoms remained fixed. An equilibrated droplet was placed at the entrance of one of the ends of the tube. The simulation setup is as shown in Fig. 5.2. The interaction between the tube atoms and monomers was described by the LJ potential with parameters $\sigma_{p t}=1.0 \sigma$ and $\varepsilon_{p t}=0.5 \varepsilon$. Temperature of the droplet was kept constant at $T=1.0 k_{B} T / \varepsilon$ using the Nosé-Hoover thermostat with a damping parameter of $10 \tau$ specified as the relaxation period for the temperature. The equations of motion were integrated via the velocity Verlet algorithm with a timestep of $0.01 \tau$.

\subsubsection{Droplets with 8000 Monomers}

We first report the results for an 8000 monomer droplet and later compare them with the results for a larger droplet with 16000 monomers. For a droplet of fixed size, the tube radius was varied systematically to determine the critical tube radius, $R_{t c}$, below which no absorption takes place. A number of simulations were run for different tube radii ranging from $R_{t}=4.26 \sigma$ to $R_{t}=6.69 \sigma$ and the subsequent absorption process was monitored for each of the cases. From these simulations we were able to narrow down the critical tube radius region and here we only focus on results from this region. The absorption process was 
monitored by recording the height of the liquid meniscus inside the tube and the radius of the protruding droplet as a function of time. The results for the $N=20$ case are shown in Fig. 5.6. For a smaller tube radius of $R_{t}=5.10 \sigma$,

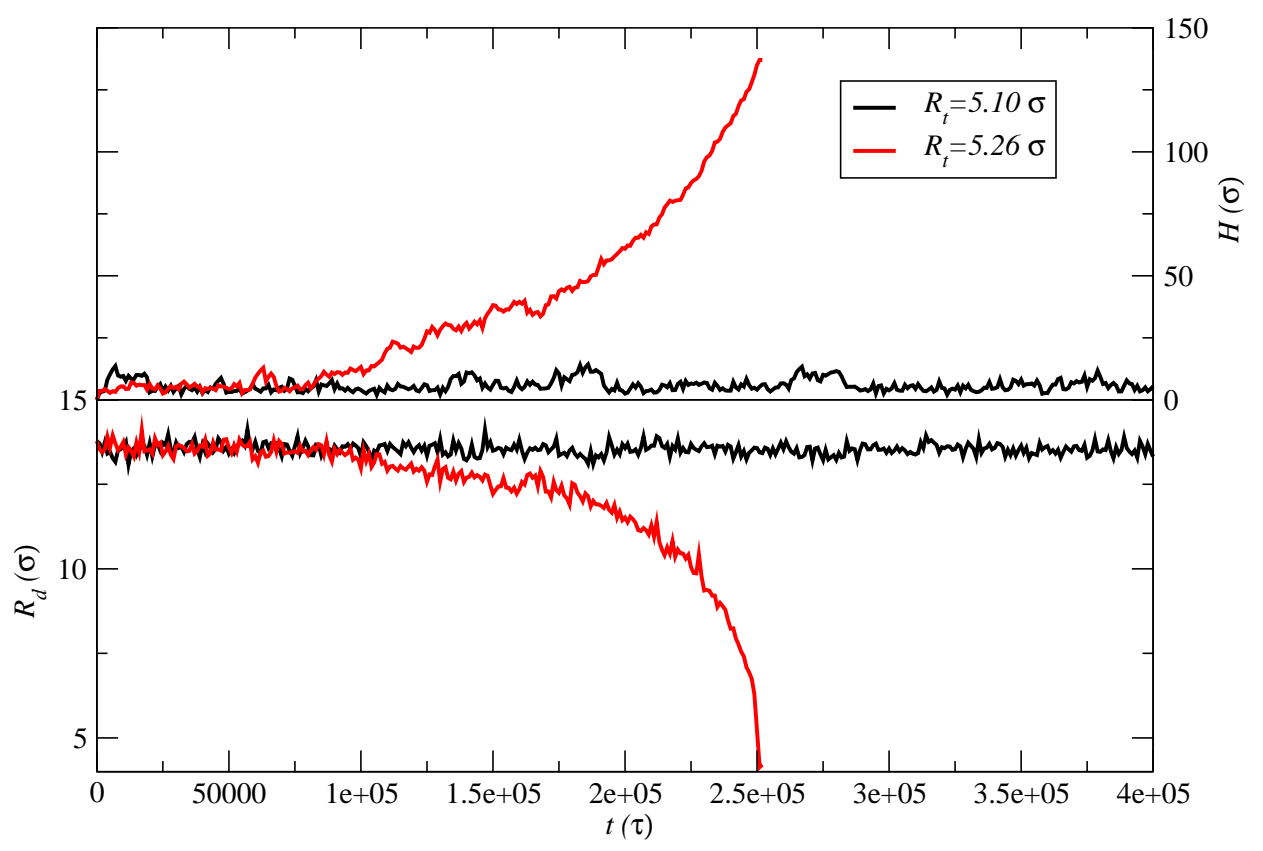

Figure 5.6: Upper section of the figure: the meniscus height; lower section of the figure: radius of protruding droplet, are plotted as a function of time for two different radii of the tube, for an 8000 monomer droplet with $N=20$ chains.

the radius of the protruding droplet and height of the liquid meniscus does not change over time. But for a slightly larger radius of $R_{t}=5.26 \sigma$ the droplet gets absorbed into the tube, once the system overcomes the initial energy barrier for penetration. Corresponding to the capillary absorption, the height of the meniscus increases and the radius of the protruding droplet decreases. In order to determine the critical cut-off radius below which there is no absorption, the simulation with smaller tube radius of $R_{t}=5.10 \sigma$ was run for much longer. Yet there was no absorption. This indicates that the critical tube radius for $N=20$ for 8000 monomer droplet is $R_{t c}=5.26 \sigma$.

To test the applicability of the Lucas-Washburn description to our systems we fit the meniscus height data to [144]:

$$
H(t)=\left(\frac{\gamma\left(R_{d}(t)+4 b\right) \cos \theta_{c}}{2 \mu} \sqrt{t}\right)
$$

where $\gamma$ is the surface tension, $R_{d}(t)$ is the radius of the protruding droplet at time $t, b$ is the slip length, and $\mu$ is the viscosity of the fluid. The initial portion of the meniscus height data were ignored as the Lucas-Washburn description does 
not account for inertial forces at initial times. The fit to the meniscus height for $R_{t}=5.26 \sigma$ was found to be in poor agreement with Eq. (5.8).

During the simulation process we also calculated the average size of the chain, averaged over all chains in the droplet, in all three directions to study the impact of the absorption process on the molecular configurations. This was achieved by computing all the three components of the radius of gyration, $R_{g}$ :

$$
\left\langle R_{g x}^{2}\right\rangle=\frac{1}{N} \sum_{i=1}^{N}\left(x_{i}-X_{c m}\right)^{2} ;\left\langle R_{g y}^{2}\right\rangle=\frac{1}{N} \sum_{i=1}^{N}\left(y_{i}-Y_{c m}\right)^{2} ;\left\langle R_{g z}^{2}\right\rangle=\frac{1}{N} \sum_{i=1}^{N}\left(z_{i}-Z_{c m}\right)^{2},
$$

where $x, y$, and $z$ are components of the monomer position vectors and $X_{c m}, Y_{c m}$, and $Z_{c m}$ are the coordinates of the centre of mass of the chain. The average behaviour of each of these components, averaged over all chains, reflects the effect of underlying dynamics on the macromolecular nature of the fluid. Each of these quantities are plotted as a function of time for $R_{t}=5.10 \sigma$ and $5.26 \sigma$ in Figs. 5.7 and 5.8 The average size of the chains remains unchanged over the whole

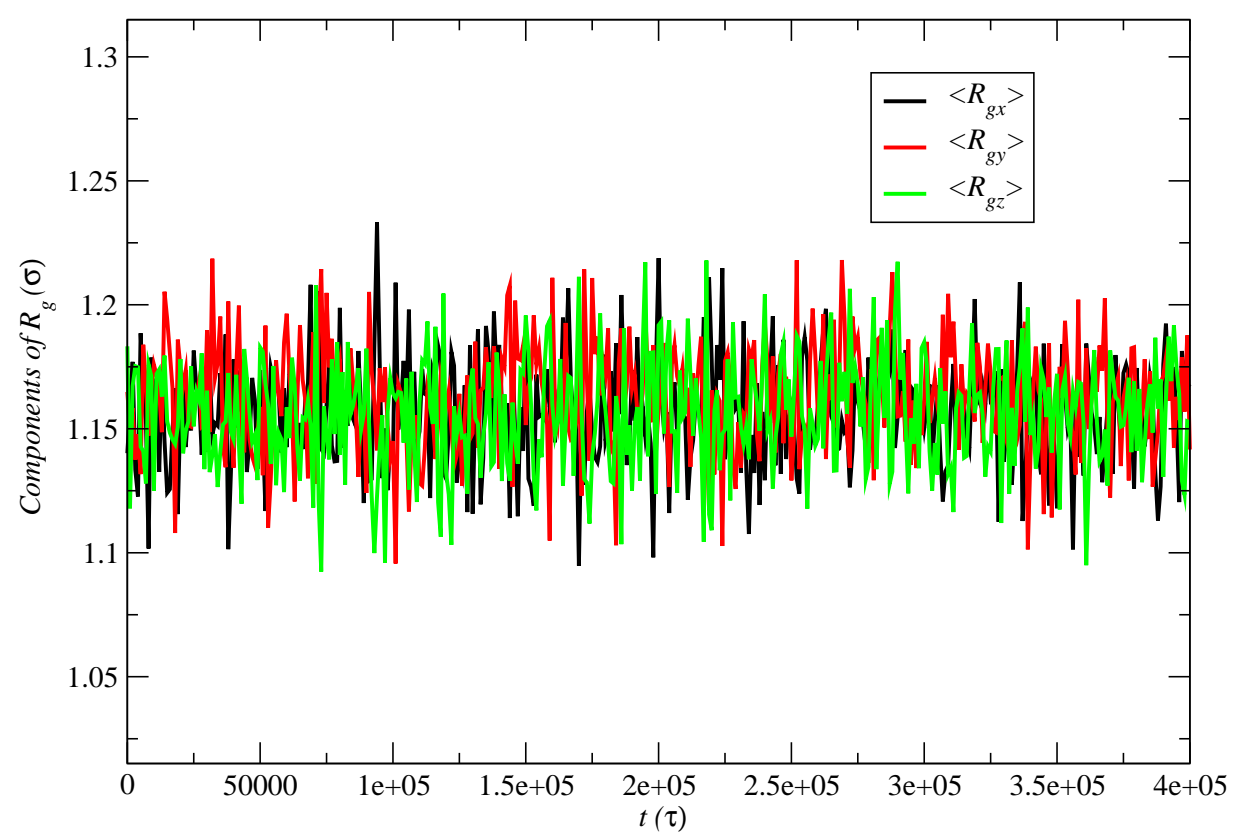

Figure 5.7: The average components of $R_{g}$ are plotted as a function of time for $R_{t}=5.10 \sigma$ for the 8000 monomer droplet.

period for $R_{t}=5.10 \sigma$. In the case of $R_{t}=5.26 \sigma$, the $R_{g y}$ component of the radius of gyration increases during the absorption process whereas the chains simultaneously shrink in the other two directions. This corresponds to elongation of the chain in the direction of the tube and compression in the other two directions.

We also investigate the effect of absorption on the potential energy which 


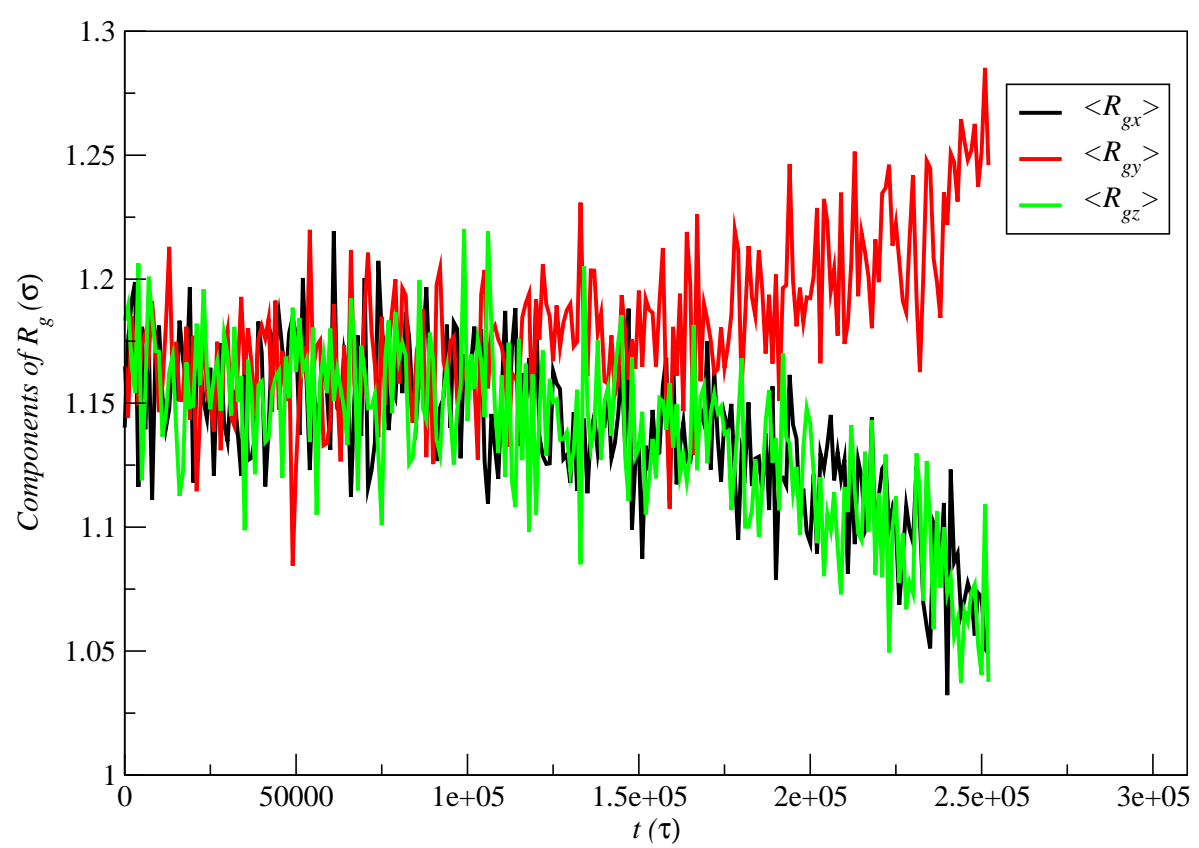

Figure 5.8: The average components of $R_{g}$ are plotted as a function of time for $R_{t}=5.26 \sigma$ for the 8000 monomer droplet.

accounts for both bond interactions due to bonded entities and LJ-type interactions between non-bonded entities. The results for the above two cases are presented in Fig. 5.9. The potential energy was approximately constant in the

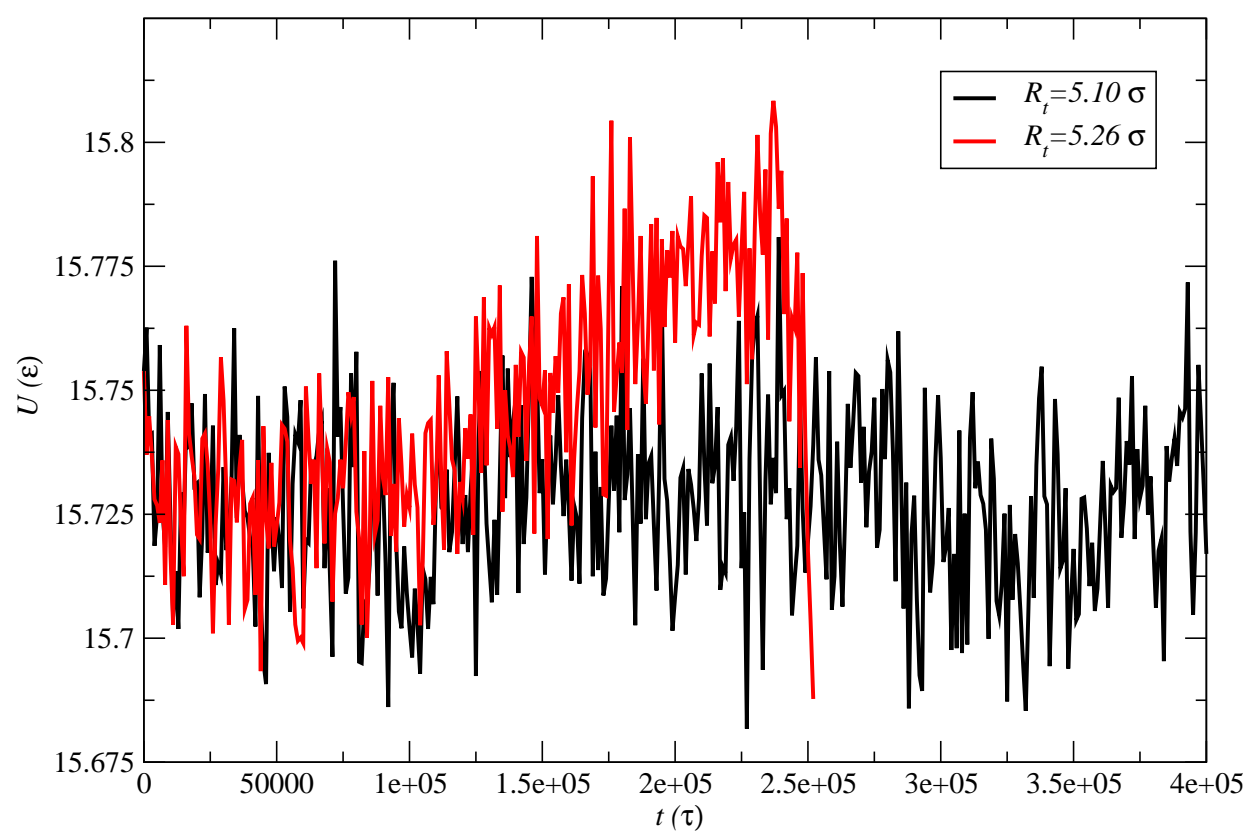

Figure 5.9: The potential energy behaviour for the 8000 monomer droplet for different tube radii.

case of $R_{t}=5.10 \sigma$ whereas it increases in the case of $R_{t}=5.26 \sigma$ during the absorption. This rise coincides with the increase trend observed in $R_{g y}$ shown in 
Fig. 5.8. Thus we confirm the increase in the chain size parallel to the tube axis, due to deformation of chains, causes an increase in the potential energy. (Note, not all of this increase is due to chain deformation, some still can be attributed to the changing shape of the droplet.)

Similar calculations were carried out for an 8000 monomer droplet made up of $N=200$ chains, to examine the effect of molecular size on the whole process. The meniscus height and the radius of the protruding droplet for this case are plotted in Fig. 5.10. Several interesting outcomes can be observed from this plot.

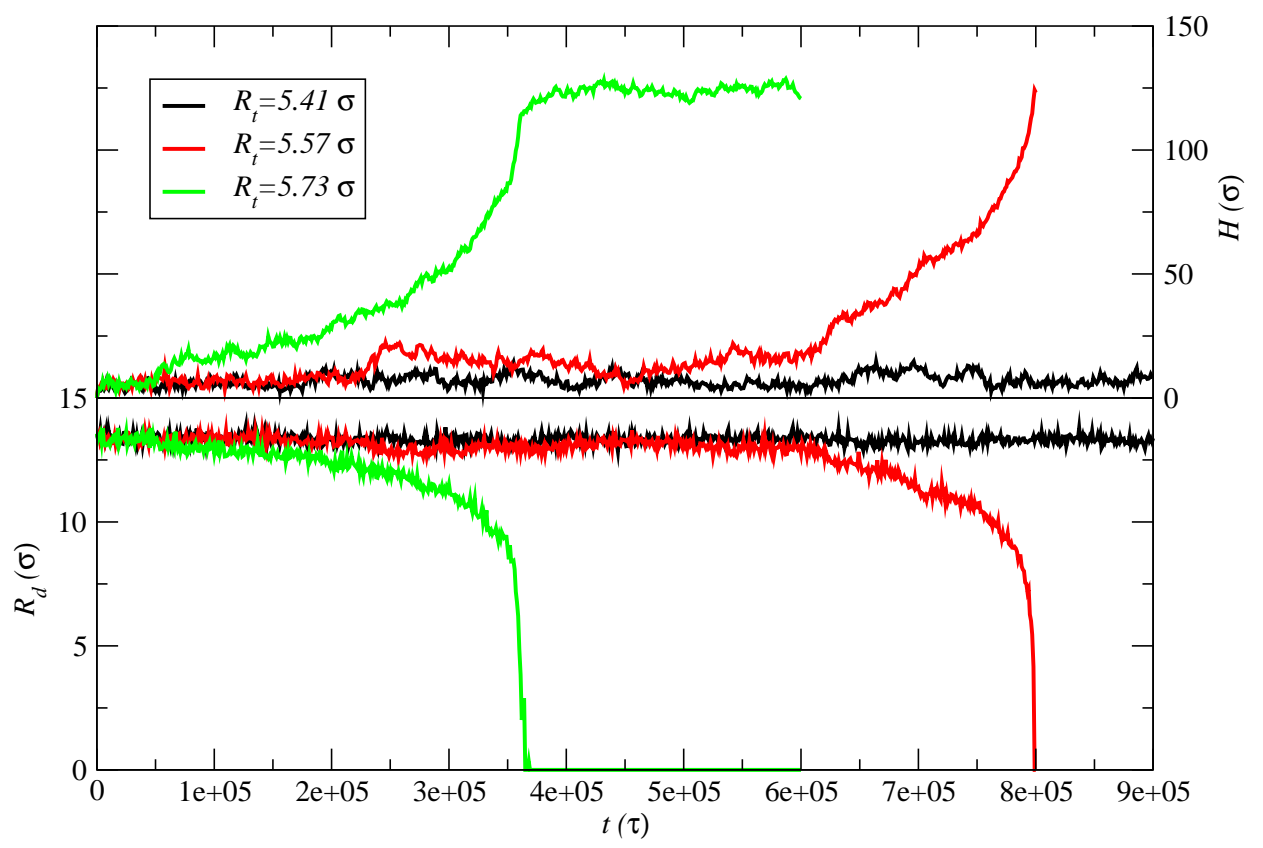

Figure 5.10: The meniscus height and the radius of the protruding droplet as a function of time for the 8000 monomer droplet with $N=200$ chains, for different tube radii.

For tubes smaller than $R_{t}=5.57 \sigma$ there was no absorption, within the time scale of the simulation runs. The absorption process was almost spontaneous for the case of $R_{t}=5.73 \sigma$ barring the initial times.

The most interesting case of all was $R_{t}=5.57 \sigma$ where the droplet sits outside the tube entrance for a very long time, during which the droplet tries to enter the tube by overcoming some sort of barrier. This is clearly evident from the meniscus height plot. The origin of this barrier can be energetic as suggested in [37] or entropic, due to conformational entropy loss, or a combination of both. During this period, we also observe partial absorption and desorption taking place. Such a process was neither present in the $N=20$ case nor was reported earlier for either Newtonian or non-Newtonian fluids. Eventually, the droplet overcomes this barrier and gets drawn up the tube. We attribute this effect to the increase in 


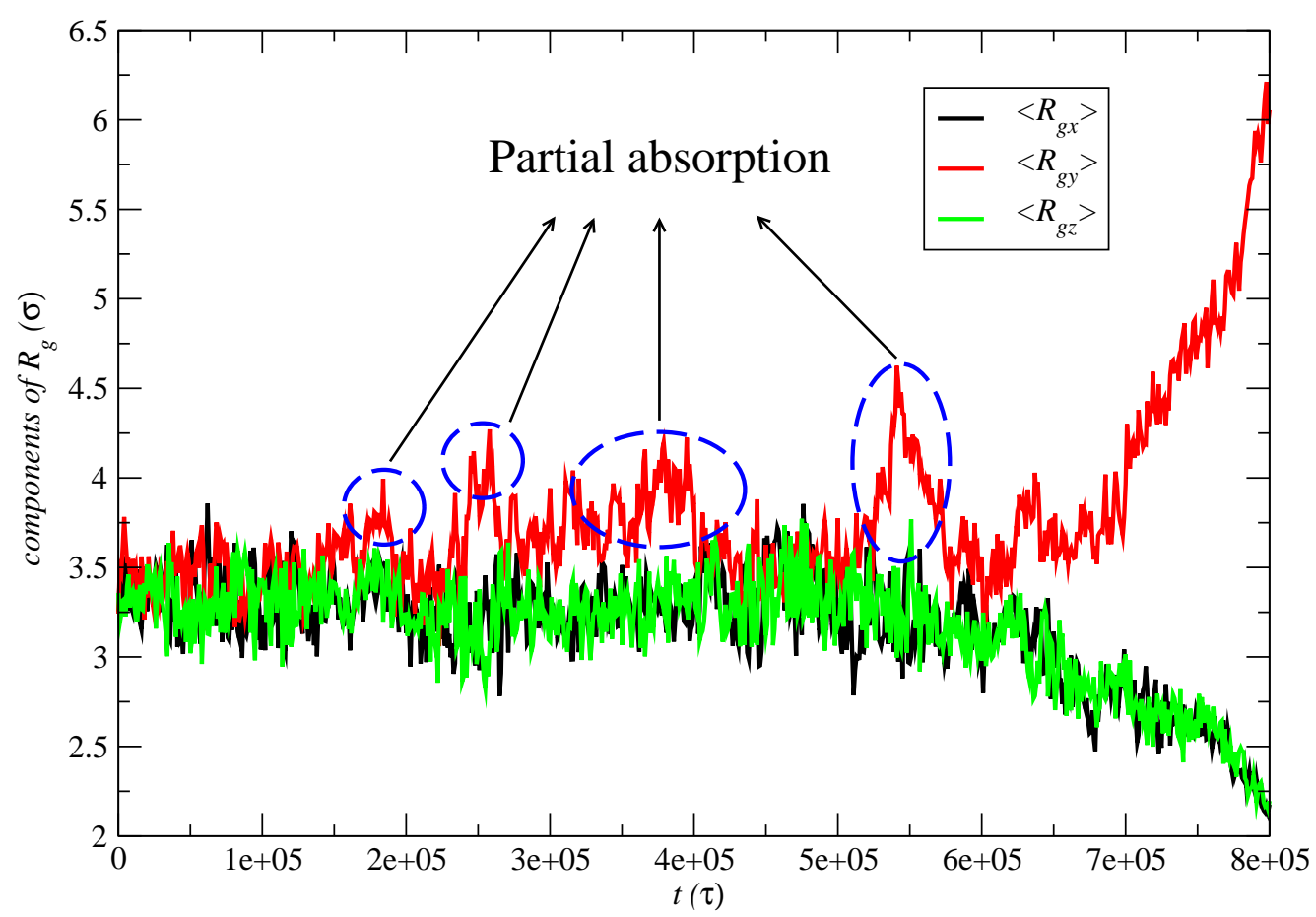

Figure 5.11: The $\left\langle R_{g}\right\rangle$ components for $R_{t}=5.57 \sigma$ and 8000 monomer droplet. The blue dashed encircled regions show the partial absorption instances.

molecular size, $N$. The average behaviour of chain size for this case is shown in Fig. 5.11. Prior to absorption, while the droplet attempts to enter the tube, fluctuations in $R_{g y}$ were observed and the trend in these fluctuations coincides with the partial absorption and desorption in the meniscus height (see Fig. 5.10). Because of the partial absorption and desorption processes, there was either an increase or decrease in $\left\langle R_{g y}\right\rangle$, corresponding to a compression or expansion of the other two components observed. Hence the average behaviour of the components of $R_{g}$ is a good measure of the underlying phenomena. Another interesting observation from this simulation was the capillary dynamics. Once again we found that the capillary dynamics observed here cannot be described by the Lucas-Washburn description (Eq. (5.8)). Moreover, the standard capillary models cannot explain the process of partial absorption and desorption observed in this case.

The average chain size measurements were also performed on the bigger tube radius $R_{t}=5.73 \sigma$ case and the results are shown in Fig. 5.12. In this particular case, we were able to study the system behaviour after complete absorption, mainly due to faster dynamics involved compared to the previous case of $R_{t}=5.57 \sigma$. Once the droplet was totally absorbed into the tube, the meniscus height remained approximately constant (refer to Fig. 5.10), during which period we monitored the average $R_{g}$ components. An interesting observation from this calculation was that after complete absorption the chains started to relax. This 


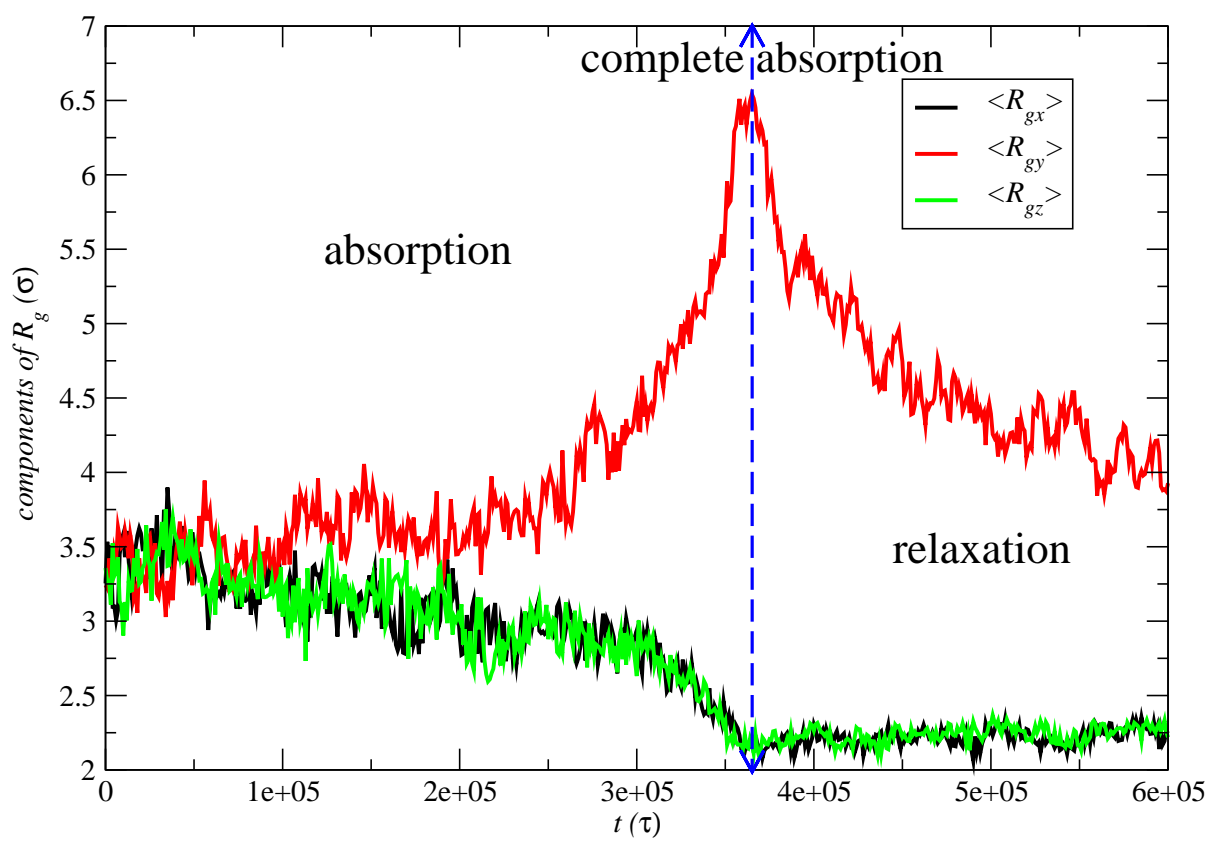

Figure 5.12: The $\left\langle R_{g}\right\rangle$ components for $R_{t}=5.73 \sigma$ and 8000 monomer droplet. The blue vertical dashed line separates the absorption process into before and after complete encapsulation.

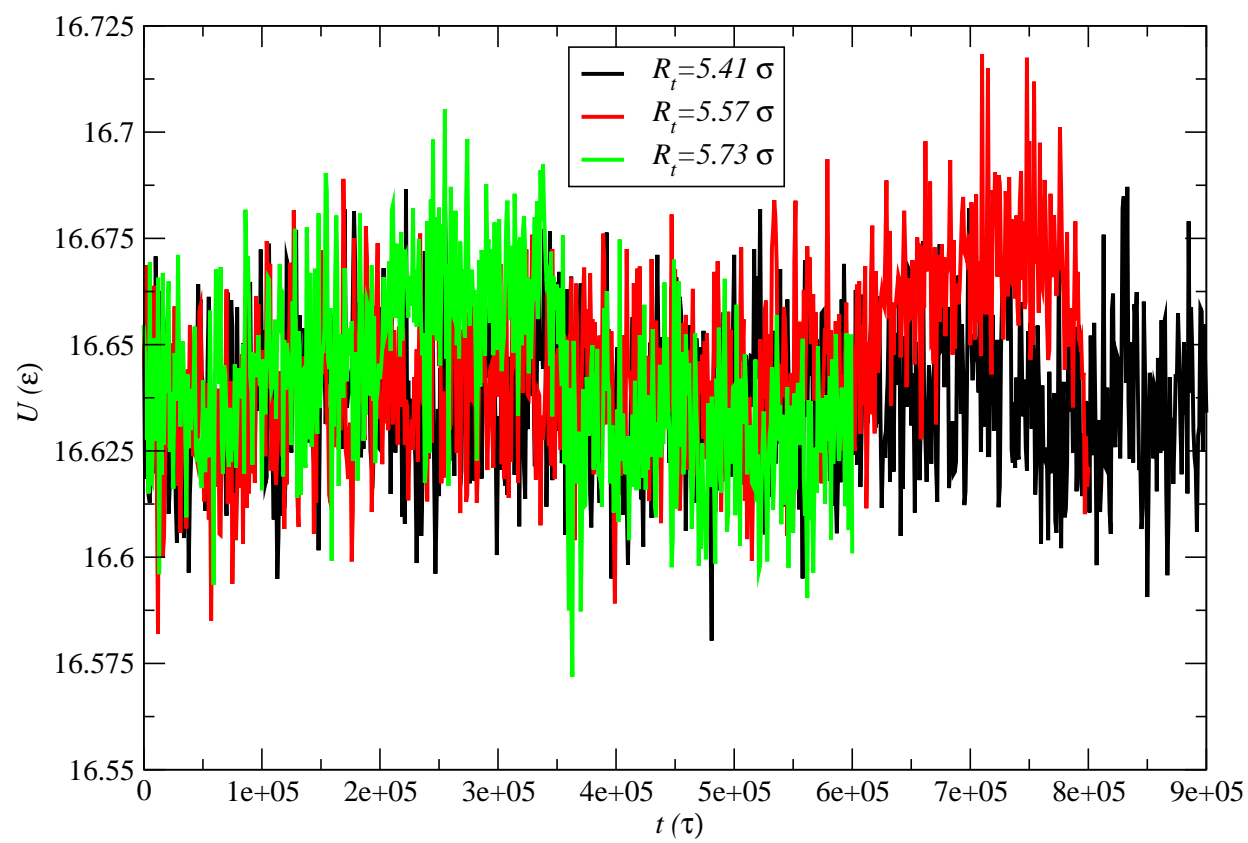

Figure 5.13: The potential energy for 8000 monomer droplet with $N=200$ as a function of time, for different tube radii. 
can be seen from the decrease in $R_{g y}$ and relatively slow increase in the other two components. In the long time limit, we expect the chains to retain their original size in the bulk (unless obstructed by the capillary walls). The potential energy measurements complement the argument for the relaxation process. The average potential energy per monomer for the 8000 monomer droplet with $N=200$ is shown in Fig. 5.13. Throughout the absorption process, depending on whether the droplet is inside or outside the tube, the potential energy either increases or decreases. In the case of $R_{t}=5.73 \sigma$, during the relaxation process the potential energy slowly decreases with time until the system attains its equilibrium state.

\subsubsection{Droplets with 16000 Monomers}

Absorption simulations were also performed for larger droplets with 16000 monomers for chain lengths 20 and 200. The critical tube radius region was identified by conducting a number of simulations for $R_{t}$ between $4.94 \sigma$ and $7.49 \sigma$. Apart from the fact that larger droplets require broader tubes for absorption, which stems from Marmur's theory, we also noticed some other interesting physics related to absorption dynamics. We shall report them one by one here. First, we present results for absorption of an $N=20$ droplet. The height of the meniscus and radius of the protruding droplet are presented in Fig. 5.14 and the critical tube radius $6.37 \sigma$. The activation time, time after which the absorption

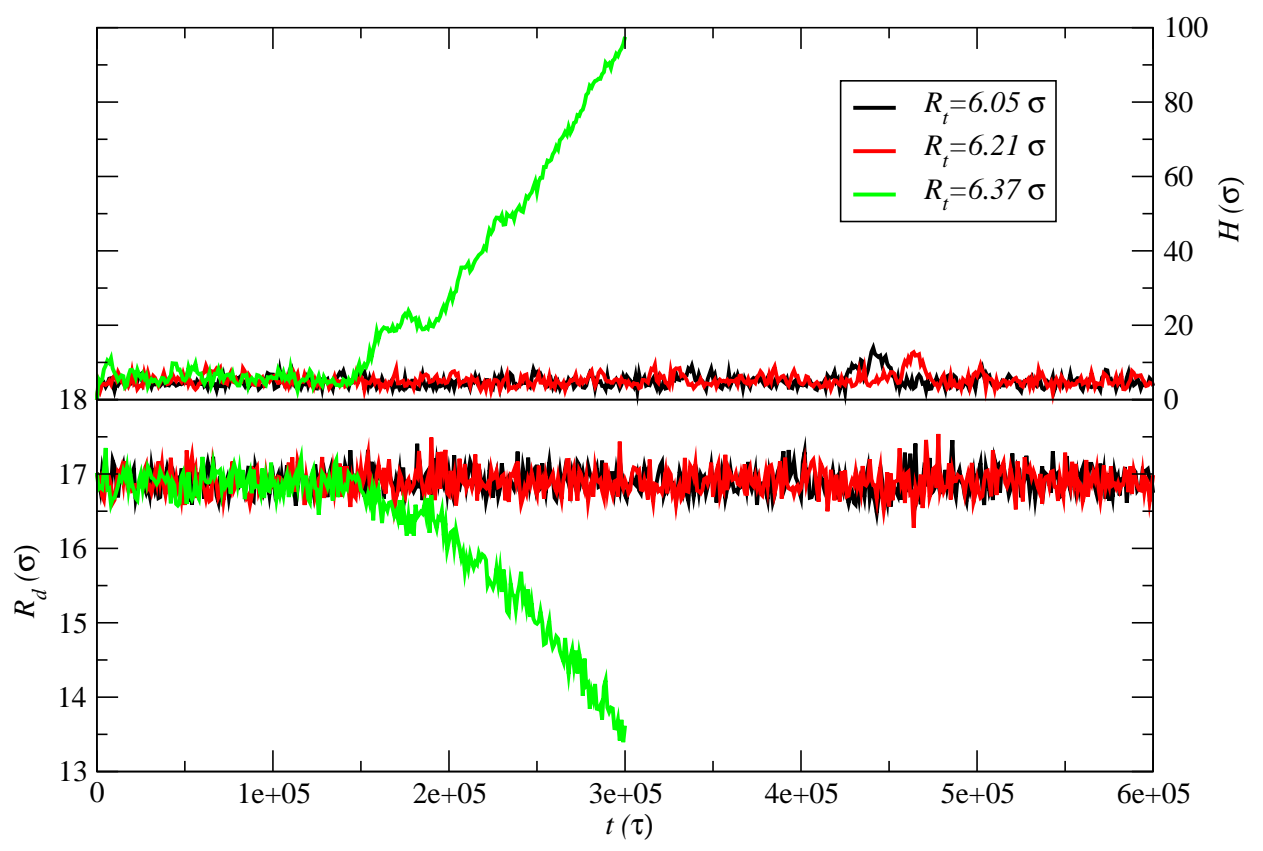

Figure 5.14: The meniscus height and radius of the protruding droplet plots for the 16000 monomer droplet with $N=20$ chains, for various tube radii.

process starts, has increased considerably compared to the 8000 monomer 
droplet for the same chain length. This could be due to an increase in curvature of the droplets resulting in weaker Laplace forces.

Results for $N=200$ droplets show remarkably different behaviour in comparison with any of the earlier cases presented. The meniscus height plots are sufficient to bring about this contrast and trends in the radius of the protruding droplet, $R_{g}$, and potential energy calculations complement its findings. The meniscus height plots for different $R_{t}$ are shown in Fig. 5.15 and the critical tube radius is $6.69 \sigma$. In the case of $R_{t}=6.53 \sigma$ throughout the simulation period

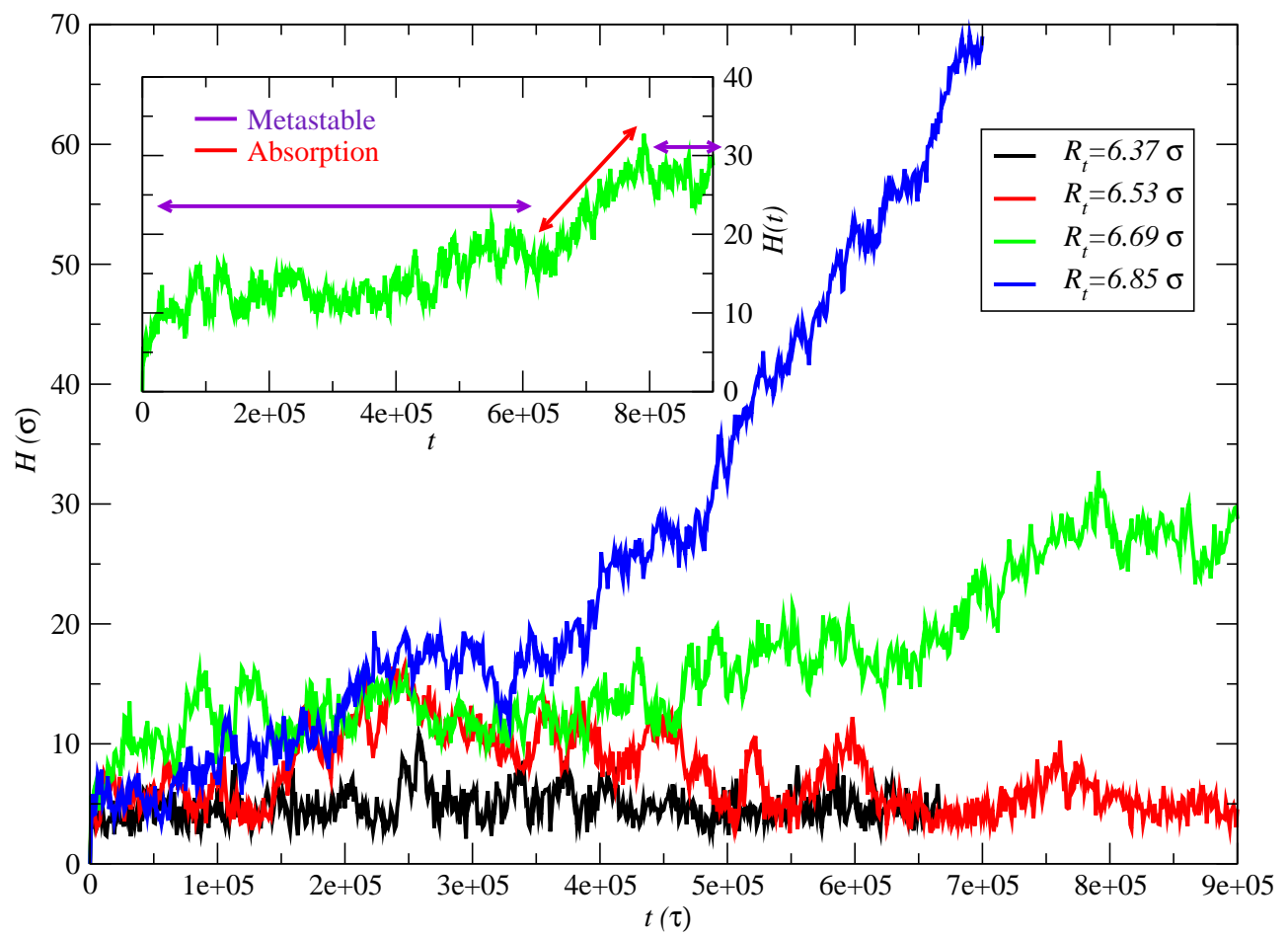

Figure 5.15: The meniscus height as a function of time for the 16000 monomer droplet with $N=200$, for various tube radii. In the inset we highlight the metastable and partial absorption regions for the case of $R_{t}=6.69 \sigma$.

the droplet was found to partially absorb and desorb without ever being able to cross the barrier. One can argue that this is purely a dynamic issue, given long enough time the droplet may eventually overcome this barrier with the help of fluctuations and capillarity phenomenon can be observed. Though we do not deny this possibility, it is not at the core of our claims, that is precise identification of the critical tube radius. The most interesting observation was the persistent partial absorption and desorption of the droplet, which has not been reported before. For a slightly larger tube of $R_{t}=6.69 \sigma$, the droplet gets stuck in multiple metastable states, for long periods of times. This scenario implies that the droplet is partially inside and partially outside for relatively long 
time periods. Such a process contrasts with existing dynamic theories aimed at explaining the capillary dynamics of simple liquids.

\subsection{Theoretical Analysis}

The MD results from the previous sections show for droplets composed of longer chains, a larger critical tube radius is required than for droplets composed of shorter chains. Specifically, we have found that the radius of gyration of the $N=20$ chains is $2.14 \sigma$ while the $N=200$ chain is $5.83 \sigma$, compared to critical tube radii of roughly $5.26 \sigma$ and $5.57 \sigma$ (respectively) for the droplet composed of 8000 monomers and $6.37 \sigma$ and $6.69 \sigma$ (respectively) for the droplet composed of 16000 monomers. We shall now attempt to understand the previous MD simulation results from a theoretical point of view - why do the droplets composed of longer chains have a larger critical tube radius? We only consider equilibrium thermodynamics here and therefore only model the initial and final, equilibrium conformations of the polymer droplets. Previously Schebarchov and Hendy [37] were able to show that non-wetting metallic droplets could be drawn up a narrow capillary tube if the droplet radius was sufficiently small. Their theoretical analysis was based on surface tension arguments (between the metallic droplet, the tube walls and the surrounding solvent) which gives rise to a Laplace pressure which assists the droplet in rising up the tube. Our analysis is based on these ideas, however, the complicating factor is that for polymer chains one needs to account for chain entropy. If a chain is confined within a narrow tube the number of possible conformations it may investigate decreases and as a result the chain's free energy increases.

In Fig. 5.3 it is shown that for $N$ small the radius of gyration of the polymer chains which make up the droplet scales roughly like a random walk chain, i.e. an exponent of 0.484 compared to the random walk exponent of 0.5 . Moreover, for almost all chain lengths sampled their radius of gyration is significantly larger than a single polymer chain (of the same length) in a poor solvent. This indicates that the chains are much more extended and investigate many more conformations than a single polymer chain in a poor solvent. Even though the droplet is in a poor solvent the individual chains which make up the droplet behave like ideal chains. This is true as long as $N$ is sufficiently small ( $N=200$ being an upper limit). We consider the situation where we have a droplet with a constant number of total monomers. Then given that the droplet is composed of chains of $N$ monomers each, we shall denote the number of chains in the droplet as $m$ and we keep $m N$ constant. We assume the chains which make up the 
polymer droplet are ideal (random-walk) chains and we measure chain entropy with respect to this state.

Consider Fig. 5.2a, in which it shown the situation when the droplet sits at the lower end of the capillary. The free energy of this state, denoted $F_{I}$, is just the surface energy of the droplet, since we measure chain entropy with respect to the ideal state. Thus we have

$$
F_{I}=\gamma_{L V} 4 \pi R_{d}^{2}+\gamma_{S V} 2 \pi R_{t} L
$$

and note that due to volume conservation $m N b^{3}=4 \pi R_{d}^{3} / 3$, yielding

$$
\frac{R_{d}}{b}=\left(\frac{3 m N}{4 \pi}\right)^{1 / 3}
$$

In the above equations, $b$ represents the monomer size, and $L$ is the length that an absorbed droplet is in contact with the tube walls.

Next we consider the situation when the droplet is completely absorbed within the capillary tube. We make the following assumptions in modelling this state:

- We assume the chains have sufficient time to disentangle and form separate blobs when moving into the tube. In doing this, they attempt to minimize the conformational entropy penalty. This, of course, may not always be kinetically possible but for sufficiently short chains (remember we only simulated absorption for chains up to $N=200$ ) is reasonable.

- The tube radius, $R_{t}$, needs to be smaller than or at least comparable to the radius of gyration of the individual chains. If the tube radius is much larger than the radius of gyration of the individual chains, then there will be no entropy penalty for entering the tube.

- We assume the chains within the droplet behave like ideal chains even though the whole droplet is in a poor solvent. Our MD simulations validate this assumption (for chains that are short enough).

A schematic of a fully absorbed droplet is shown in Fig. 5.2c. The volume of this droplet is

$$
V_{I I}=\pi R_{t}^{2} L-\frac{4}{3} \pi R_{t}^{3}\left(\frac{1-\frac{3}{2} \sin \theta_{c}+\frac{1}{3} \sin ^{3} \theta_{c}}{\cos ^{3} \theta_{c}}\right)
$$

and of course this volume is just $m \mathrm{Nb}^{3}$. Using this equation we can eliminate $L$ 
in favour of $R_{t}$. The surface energy associated with this droplet shape is

$$
F_{I I}^{S}=\gamma_{S L} 2 \pi R_{t} L+\gamma_{L V} \frac{4 \pi R_{t}^{2}}{1+\sin \theta_{c}}
$$

Now we need to determine the conformational energy penalty (of the polymer chains) for entering the narrow capillary. To determine this entropy penalty we use the arguments of Grosberg and Khokhlov [53]. (The argument is a scaling type analysis, meaning numerical pre-factors are ignored.) The energy cost for an ideal chain to be confined to a tube of radius $R_{t}$ is based on a collisional argument. The ideal chain takes $K$ steps to traverse from one side of the tube to the other, where $R_{t} \sim K^{1 / 2} b$. Every time the chain hits the tube wall it incurs an energy penalty of $k_{B} T$. A chain composed of $N$ monomers is composed of $N / K$ such random walks and therefore has $(N / K-1)$ collisions. Since $K=\left(R_{t} / b\right)^{2}$ the free energy of confinement is

$$
F_{\text {confined }}=k_{B} T\left(N\left[\frac{b}{R_{t}}\right]^{2}-1\right) \text {. }
$$

We should mention the $N$ in the above equation does not directly correspond to the chain lengths we used in the MD section. The MD represents a coarse-grained model where several base units are grouped into each monomer. As a result the $N$ in Eq.(5.14) is of the order of $10^{3}-10^{6}$ and so the last term of minus one is negligible compared to the first term. However, we will leave it here since it has some significance (see below). Recall we have $m$ chains which make up this droplet so the total entropic energy penalty, $F_{I I}^{P}$, is

$$
F_{I I}^{P}=m k_{B} T\left(N\left[\frac{b}{R_{t}}\right]^{2}-1\right) .
$$

We can see that as $R_{t} \rightarrow 0$ the entropy energy diverges. Moreover, if the droplet is made up of shorter chains ( $N$ decreases and $m$ increases but still $m N$ is constant) the entropical energy decreases. This is as we would expect and is due to fewer collisions per chain (as the chain length decreases). The total free energy, $F_{I I}$, for an absorbed droplet is $F_{I I} \equiv F_{I I}^{S}+F_{I I}^{P}$. Note, that this energy is valid only if the radius of gyration of individual chains which make up the droplet is comparable in size to tube radius, so that a lower bound for this energy is just $F_{I I}^{S}$. The important energy is the free energy difference between an absorbed droplet and the droplet sitting at the entrance of the capillary. Thus we define this difference as $\Delta F \equiv F_{I I}-F_{I}$. For convenience we scale this free energy difference 
by $\gamma_{L V} 4 \pi R_{d}^{2}$ to make it dimensionless and denote it as $\Delta \Phi$. It is given by (after some algebraic manipulation)

$$
\begin{aligned}
\Delta \Phi \equiv \frac{\Delta F}{\gamma_{L V} 4 \pi R_{d}^{2}}=\left(\frac{1}{3}\right)\left(\frac{R_{t}}{R_{d}}\right)^{2} & \left(\frac{1}{1+\sin \theta_{c}}+\sin \theta_{c}\right)-\frac{2}{3}\left(\frac{R_{d}}{R_{t}}\right) \cos \theta_{c}-1 \\
& +\left(\frac{k_{B} T}{\gamma_{L V} 4 \pi R_{d}^{2}}\right) m\left(N\left[\frac{b}{R_{t}}\right]^{2}-1\right) .
\end{aligned}
$$

Note, $4 \pi R_{d}^{2}$ is known and equal to $(4 \pi)^{1 / 3}(3 m N)^{2 / 3} b^{2}$. An analytically simpler, yet accurate, expression is obtained if we neglect the minus one term in the entropy. In this case we obtain

$$
\Delta \Phi=\frac{1}{3 \eta^{2}}\left(\frac{1}{1+\sin \theta_{c}}+\sin \theta_{c}\right)-\frac{2}{3} \eta \cos \theta_{c}-1+\frac{\eta^{2}}{3 \kappa\left(R_{d} / b\right)}
$$

where $\eta \equiv R_{d} / R_{t}$ and $\kappa \equiv \gamma_{L V} b^{2} /\left(k_{B} T\right)$. The absorbed state will be favoured when $\Delta \Phi<0$.

Consider the partial wetting case first, i.e. $\theta_{c} \leq \pi / 2$ and recall we are only interested in the situation where the tube diameter is smaller than the liquid droplet diameter. In this case, in the absence of a polymeric contribution (last term in Eq.(5.16)) the liquid droplet will always be absorbed fully into the capillary [37]. However, for polymer liquid droplets this is not the case, since the entropic term is positive and its presence favours the droplet sitting outside the tube. The case $\theta_{c}=\pi / 2$ is particularly instructive in this context. For large $\eta$ the surface energy terms are approximately -1 . However a polymeric droplet will not enter the tube when $\eta>\eta_{c}$ where

$$
\eta_{c}=\sqrt{3 \kappa\left(R_{d} / b\right)}
$$

This means for $R_{t} / b<\sqrt{\frac{\left(R_{d} / b\right)}{3 \kappa}}$ the polymer droplet will not be absorbed in contrast to the non-polymeric droplet. However, this corresponds to a very narrow tube, e.g., if $R_{d} / b \approx 300$ and $\kappa \approx 1$ then the tube radius below which absorption ceases is $10 b$. In Fig. 5.16 is shown the free energy for polymeric (full-lines) and non-polymeric droplets (dashed lines) versus $\eta$ for a contact angle of $\pi / 2$. For this figure we use $\kappa=1, m N=10^{6}$ and we use Eq. (5.16) for the free energy. The non-polymeric case, as explained above, is always fully absorbed while for only one polymer chain of length $10^{6}$ the critical value of $\eta$ is 13.6, while for $10^{3}$ chains each of length $10^{3}$ the critical $\eta$ is slightly larger at 13.8 . 


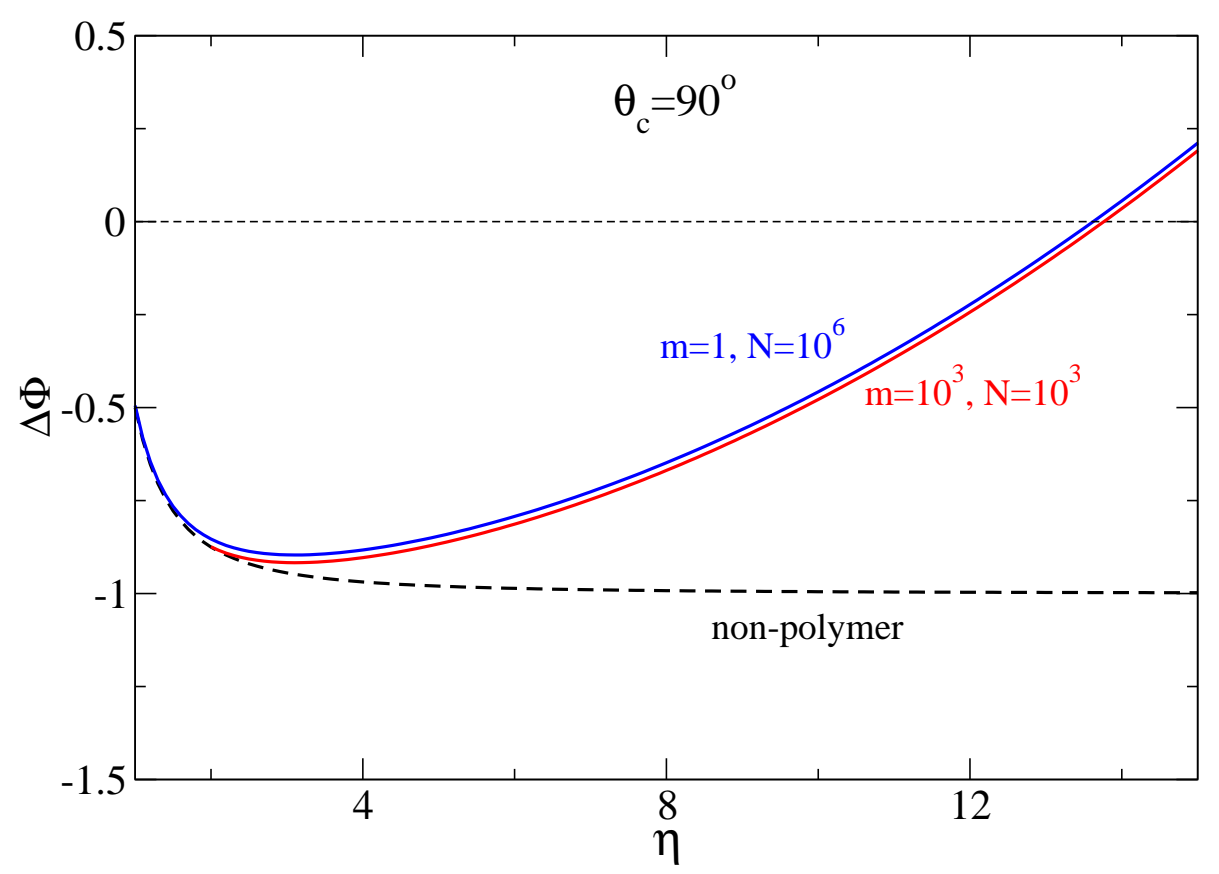

Figure 5.16: Surface energy difference for various droplet configurations for $\theta_{c}=90^{\circ}$ as a function of $\eta$.

For the non-wetting case, i.e., $\theta_{c}>\pi / 2$ yielding $\cos \theta_{c}<0$ there is only one term favouring absorption. Figure 5.17 shows the free energy for a contact angle of $103.5^{\circ}$. (Recall the contact angle from the MD simulations was in the non-wetting regime $\left(\approx 103.5^{\circ}\right)$.) In the absence of the polymeric term, all droplets below a radius of $6.35 R_{t}$ would be absorbed into the capillary. For both polymeric cases, however the critical tube radius is smaller (at $5.5 R_{t}$ for the $10^{3}$ length chains and $5.3 R_{t}$ for the $10^{6}$ length chain) and the critical $\eta_{c}$ decreases with increasing chain length. In our MD simulations for the droplet of 8000 monomers, the critical $\eta_{c}$ for short chains $(N=20)$ is 2.9 , which for longer chains $(N=200)$ is 2.7. For the droplet of 16000 monomers, the critical $\eta_{c}$ for short chains $(N=20)$ is 2.95 , which for longer chains $(N=200)$ is 2.8 . Thus the theoretical results agree with our MD simulations qualitatively in that both MD simulations and theory predict (i) a decrease in critical $\eta$ with longer chains and (ii) when a simple molecular fluid droplet would be absorbed, the polymeric droplet remains outside the tube. This difference can only be attributed to the entropy penalty the chains must overcome when entering a restricted domain. 


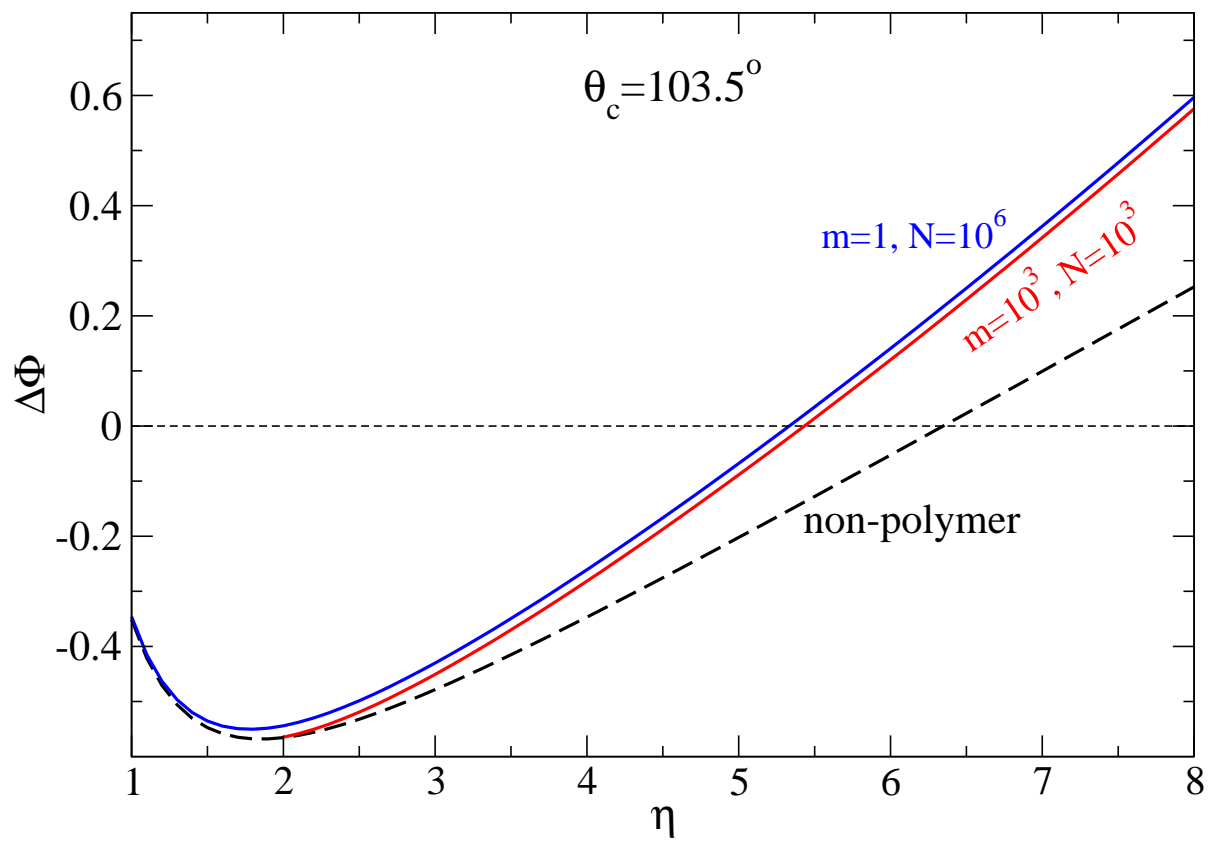

Figure 5.17: Surface energy for various droplet configurations for $\theta_{c}=103^{\circ}$ as a function of $\eta$.

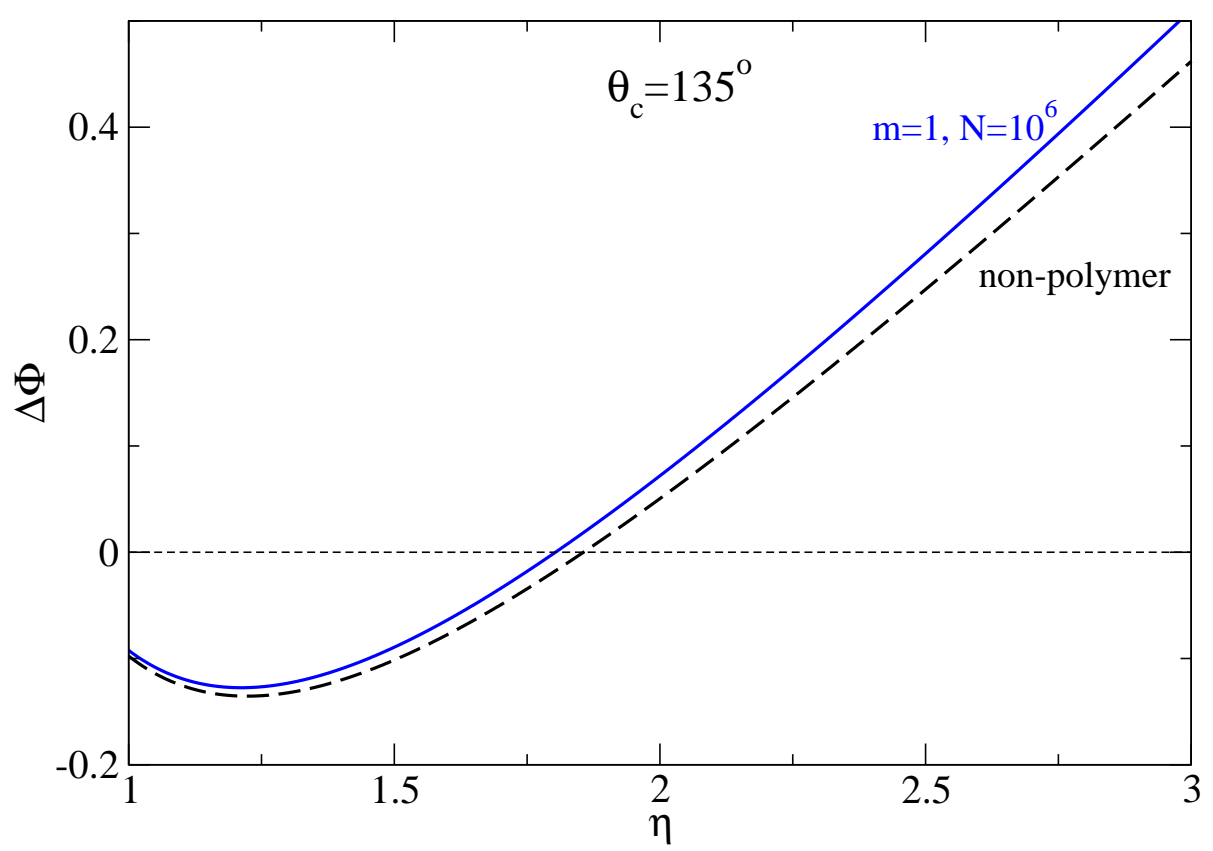

Figure 5.18: Surface energy for various droplet configurations for $\theta_{c}=135^{\circ}$ as a function of $\eta$. 
Finally we present results for the case of a contact angle of $135^{\circ}$ (Fig. 5.18). The results generally follow the previous cases. However, an important difference now is that the surface tension term in much more important. As a result the regime where droplets will be absorbed is very small and furthermore the critical $\eta_{c}$ for the polymeric and non-polymeric droplets become very close. In this case we do not consider a droplet made up of chains of length $10^{3}$ because their radius of gyration is smaller than the tube radius for $\eta<2$.

\subsection{Conclusions}

We have presented MD simulations for absorption of polymer droplets, made up of many small chains, into narrow capillaries. The chains behave (for sufficiently short chains) like ideal chains since most of the chains are surrounded by other polymer chains. By tuning the MD interaction parameters we were able to simulate a system with a contact angle of roughly $103.5^{\circ}$, corresponding to a non-wetting droplet. We then simulated the absorption of droplets composed of shorter and longer chains into a narrow capillary and found in general the longer chains could not enter sufficiently narrow tubes. We attribute this phenomena to chain entropy, the longer chains lose more conformation entropy on entering narrow tubes. Moreover, we also found that capillary dynamics of polymer droplets in such narrow capillaries cannot be explained using the existing models. The MD simulations and theory presented agree qualitatively. The theory also suggests for sufficiently narrow tubes or long polymer chains, polymer droplets which wet the tube walls, may not enter the tube. This is in contrast to a simple wetting liquid which always absorbs into the tube. 


\section{Chapter 6}

\section{SPH Simulations of Polymer Blends}

Here we study phase separation in polymer blends using a numerical technique called Smoothed Particle Hydrodynamics (SPH) [145]. Polymer blends have many industrial applications [146, 147] and are useful in studying phase separation phenomena [148]. These systems are also interesting from the viewpoint that the theoretical concepts developed for studying them can be extended to other polymer systems such as diblock copolymers [149].

A polymer blend is a mixture of two or more different types of polymers. These systems are often investigated with the purpose of producing materials that have combined features of all the constituent polymers. Such materials are useful in circumstances where the blend possesses desired properties that are absent in any of its constituent polymers, in their pure states. For example, blending an elastomer with a rigid polymer produces a material that is rigid yet less brittle. Here we are concerned with binary mixtures of homopolymer blends and hence we shall restrict our discussion to these types of blends only.

Although, ideally, it is desirable to have a polymer blend that can mix homogeneously, most polymer blends do not mix i.e., they phase separate [150]. Based on temperature, composition of the blend and chain lengths of the constituent molecules, polymer blends exhibit different phase behaviour. Therefore, knowledge of phase separation behaviour of polymer blends is essential from the perspective of their potential applications.

Numerical methods deployed for studying phase separation phenomena in polymer systems are mostly lattice- or grid-based [149, 44]. These methods either use lattice Monte Carlo or solve the time-dependent Ginzburg-Landau equation on a grid using finite difference techniques. However, lattice models 
have disadvantages such as (a) anisotropy effects, stemming from the grid-based nature of these methods, which can be significant in thin film studies and (b) inefficiency in modelling dense polymer systems [44, 45], for example. Moreover, these methods can be computationally highly inefficient and/or expensive in modelling systems with free surfaces. Thin films of polymer blends, generally, are prepared by spin-casting on substrates, where the top surface freely deforms, are interesting from technological and scientific viewpoints [41, 42, 43, 151, 152]. Block copolymers are other polymer systems that self-assemble and form more complex morphologies, than polymer blends, on nanoscale, which are of great interest in technology [153, 154, 155]. These systems also exhibit interesting behaviour on substrates, where the top surface freely deforms and this leads to undulations or island-hole structures at the free surface [156, 157, 47, 48, 49]. Grid-based methods are not efficient for modelling such systems. As mentioned earlier, the theoretical framework of polymer blends can be extended to study diblock copolymer systems [149]. On the other hand, atomistic simulation methods such as the MD or off-lattice methods can tackle the free surface problems mentioned above, in principal, but they can be computationally very intensive [44]. We employ the SPH method for studying polymer blends, which we believe has the potential to address most of the above listed issues.

The chapter is organized as following. In section 2 we discuss the thermodynamics of mixing in polymer blends and its relation to the model used in our simulations. In section 3 we introduce the basic concepts of the SPH method; and in section 4 its application to study phase separation in polymer blends. In section 5 we focus on tools that are used to improve the efficiency of the algorithms. In section 6 we present the results from SPH simulations of polymer blends. Finally in section 7 we give conclusions from this work and also briefly discuss future work.

\subsection{Thermodynamics of Phase Separation}

Here we give a brief review of the thermodynamic treatment of polymer blends, originally due to Flory [4] and Huggins [5] and now given in standard texts on polymer physics $[119,46,53]$. Under given conditions, if a blend prefers to mix and form a single homogeneous phase then the mixture is said to be miscible. On the other hand if the system phase separates into regions that are rich in either species then the blend is said to be immiscible. The study of phase behaviour of a blend as a function of temperature and composition gives us the phase diagram of the system.

The phase behaviour of any mixture can be studied by measuring the change 
in free energy upon mixing. To estimate this quantity, let us consider two homopolymer systems that are initially separate and subsequently brought into contact with each other. Whether the blend prefers to mix, leading to a homogeneous phase, or phase separates depends on the sign of change in free energy given by

$$
\Delta F_{m i x}=\Delta U_{m i x}-T \Delta S_{m i x}
$$

where $\Delta U_{\text {mix }}$ and $\Delta S_{\text {mix }}$ are the change in enthalpy and entropy between mixed and pure states, respectively. By pure states we refer to both $A$ and $B$ fluids prior to when they were brought in contact. If $\Delta F_{\text {mix }}<0$, the system prefers to be homogeneous and if $\Delta F_{\text {mix }}>0$ then it prefers to phase separate, with the aid of fluctuations. We shall attempt to derive expressions for $\Delta S_{\text {mix }}$ and $\Delta U_{m i x}$.

Let us consider a polymer blend with two types of polymers, $A$ and $B$. For simplicity, we use a lattice model where each lattice site is occupied by either an $A$ or a $B$ monomer. In the above statement it is implicitly assumed that both monomers are identical in size which is not true in general. It also implied that the system is incompressible. Though this models is an over-simplification of the problem it manages to capture the essential physics of phase separation.

First we calculate the entropy of mixing. Note, here we are aiming to obtain an expression for the combinatorial part of the entropy. We do not account for configurational entropy that arises from the macromolecular nature of polymers. We also ingore the non-combinatorial entropy that arises from changes in the volume upon mixing or in the presence of surfaces [158]. This non-combinatorial entropy dominates over enthalpy at elevated temperatures. Assuming that the system is already in a homogeneous state, the entropy per monomer is

$$
s_{\text {mix }}=k_{B} \ln \Omega_{m i x}
$$

where $\Omega_{\text {mix }}$ is the number of ways a monomer can be arranged on the lattice which in this case is equal to number of lattice sites, $n$. Hence,

$$
s_{\text {mix }}=k_{B} \ln n
$$

If the volume fractions of $A$ and $B$ polymers in the system are denoted by $\phi_{A}$ and $\phi_{B}$, then the number of $A$ monomers is equal to $n \phi_{A}$ and the number of $B$ monomers is equal to $n \phi_{B}$. The entropy per monomer in pure states, for the A-specie is $s_{A}=k_{B} \ln \Omega_{A}=k_{B} \ln n \phi_{A}$, and similarly for $s_{B}$. Here $\Omega_{A}$ is the number of ways in which a monomer belonging to the A-species can be arranged 
on its respective lattice with $n \phi_{A}$ sites. Therefore, the entropy of mixing per monomer for $A$ polymers is $\Delta s_{A}=s_{\text {mix }}-s_{A}=-k_{B} \ln \phi_{A}$. If the blend consists of $n_{A}, A$ monomers and $n_{B}, B$ monomers then the total change in entropy of the system is given by

$$
\Delta S_{\text {mix }}=n_{A} \Delta s_{A}+n_{B} \Delta s_{B}
$$

This equation is valid for any binary mixture. For a polymer blend with molecular weights of the constituent polymers $N_{A}$ and $N_{B}$ the above equation can be rewritten to calculate $\Delta S_{\text {mix }}^{m}$ per molecule as

$$
\Delta S_{m i x}^{m}=\frac{n_{A}}{N_{A}} \Delta s_{A}+\frac{n_{B}}{N_{B}} \Delta s_{B}
$$

Using $n_{A}=n \phi_{A}$ and $n_{B}=n \phi_{B}$, we obtain $\Delta s_{\text {mix }}$ per monomer (or per lattice site)

$$
\Delta s_{\text {mix }}=-k_{B}\left[\frac{\phi_{A}}{N_{A}} \ln \phi_{A}+\frac{\phi_{B}}{N_{B}} \ln \phi_{B}\right]
$$

Since $\phi_{A}$ and $\phi_{B}$ are always less than 1, the entropy of mixing is always positive and thus promotes mixing. With increasing temperature, $T \Delta s_{\text {mix }}$ also increases which results in lowering the free energy of mixing. One important point to note here is the molecular weight dependence of $\Delta s_{\text {mix }}$. For binary solutions and low molecular weight system $\Delta s_{\text {mix }}$ is large. But for high molecular weight blends, which is the case for most polymer blends, $\Delta s_{\text {mix }}$ becomes significantly smaller. The implications of this on the phase separation will be discussed later in the section.

Now we calculate the enthalpy of mixing. We assume that the interactions for any monomer are restricted only to its immediate neighbours and are also limited to pair-wise interactions only. Let us denote the pair-wise interaction energies between $A-A, A-B$ and $B-B$ monomers by $\varepsilon_{A A}, \varepsilon_{A B}$ and $\varepsilon_{B B}$ respectively. Under the mean field approximation, in a homogeneous mixture, at any site, the probability of finding an $A$ monomer is $\phi_{A}$ and the probability of finding a $B$ monomer is $\phi_{B}$. Therefore, the enthalpy of mixing per site in homogeneous phase is

$$
u_{m i x}=\frac{z}{2}\left[\phi_{A}^{2} \varepsilon_{A A}+\phi_{B}^{2} \varepsilon_{B B}+2 \phi_{A} \phi_{B} \varepsilon_{A B}\right] .
$$

Here $z$ is the coordination number of the lattice. The factor of a half in Eq. (6.7) accounts for double counting. 
The enthalpy per monomer in pure states for each of the species is

$$
u_{A}=\frac{z}{2} \phi_{A} \varepsilon_{A A} ; u_{B}=\frac{z}{2} \phi_{B} \varepsilon_{B B} .
$$

From Eqs. (6.7) and (6.8), the enthalpy of mixing is

$$
\Delta u_{m i x} \equiv u_{m i x}-\left(u_{A}+u_{B}\right)=\frac{z}{2}\left[\left(\phi_{A}^{2}-\phi_{A}\right) \varepsilon_{A A}+\left(\phi_{B}^{2}-\phi_{B}\right) \varepsilon_{B B}+2 \phi_{A} \phi_{B} \varepsilon_{A B}\right] \text {. }
$$

Under the conditions of incompressibility and no change in volume upon mixing, we have $\phi_{A}+\phi_{B}=1$. However in real systems there is a small, but non-zero, change in the volume upon mixing, which we ignore here. Using the above approximations in Eq. (6.9) we arrive at

$$
\Delta u_{m i x}=z \phi(1-\phi)\left[\varepsilon_{A B}-\frac{1}{2}\left(\varepsilon_{A A}+\varepsilon_{B B}\right)\right]
$$

where $\phi \equiv \phi_{A}$. The Flory interaction parameter defined by

$$
\chi=\frac{z}{k_{B} T}\left[\varepsilon_{A B}-\frac{1}{2}\left(\varepsilon_{A A}+\varepsilon_{B B}\right)\right],
$$

contains information regarding the characteristic nature of the interactions. If $\chi<0$ then unlike molecules would like to mix while if $\chi>0$ then interactions between like molecules is favoured leading to phase separation of the blend. Substituting for $\chi$ in Eq. (6.10) we get

$$
\Delta u_{m i x}=\chi \phi(1-\phi) k_{B} T
$$

Combining Eqs. (6.6) and (6.12), the free energy of mixing per lattice site is

$$
\Delta f_{m i x}=k_{B} T\left[\frac{\phi}{N_{A}} \ln \phi+\frac{1-\phi}{N_{B}} \ln (1-\phi)+\chi \phi(1-\phi)\right] .
$$

From Eq. (6.13) we can study phase behaviour of any mixture. For low molecular blends or solutions, $N_{A}$ and $N_{B}$ are small. Hence the entropic contribution dominates the phase behaviour. For high molecular weight polymer blends the entropic contribution becomes small and the trade-off between the entropic and enthalpic contributions regulates the phase separation process.

In the upper half of Fig. 6.1, we have plotted the $N \Delta f_{\text {mix }} / k_{B} T$ as a function of composition $\phi$ for a symmetric polymer blend $\left(N_{A}=N_{B}=N\right)$ for a particular value of $\chi N$. It is reasonable to choose $\chi N$ as the suitable parameter to study the 
phase behaviour. This parameter includes temperature and molecular weight, both of which influence the underlying phase separation process. Note that this is not the case for solvent-solvent mixtures where just the $\chi$ parameter is sufficient to describe the phase separation mechanism. $\Delta f_{\text {mix }}$ has two minima at

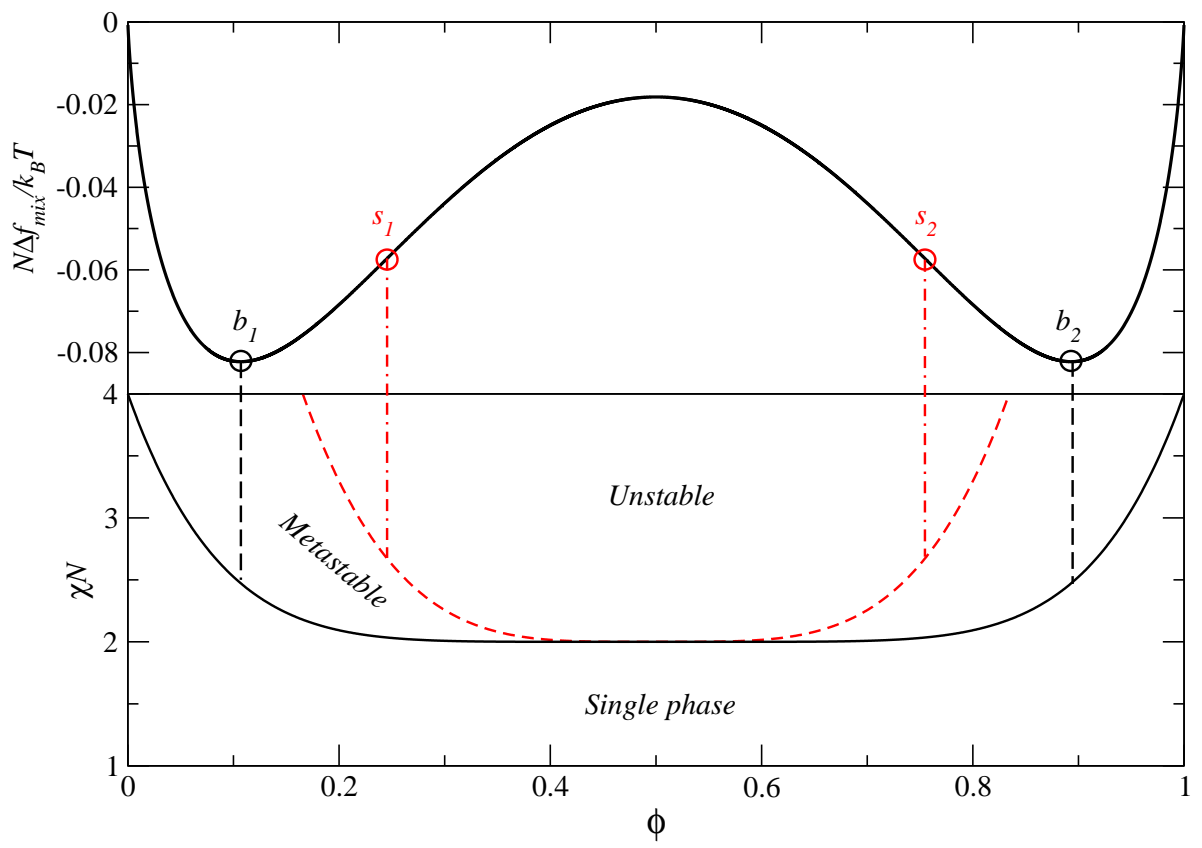

Figure 6.1: In the upper graph the free energy of mixing as a function of composition is plotted. The points $b_{1}$ and $b_{2}$ represent coexisting phases. Between points $s_{1}$ and $s_{2}$ the system is metastable with respect to fluctuations in the composition. In the lower graph, the black curve is obtained from projecting $b$ s from the free energy curve and the red curve is obtained from projecting ss curve, as a function of $\chi N$.

points $b_{1}$ and $b_{2}$ with a maxima in between. The first derivative of free energy with respect to $\phi$ is equal at these two points, i.e.,

$$
\left(\frac{\partial \Delta f_{m i x}}{\partial \phi}\right)_{b_{1}}=\left(\frac{\partial \Delta f_{m i x}}{\partial \phi}\right)_{b_{2}} .
$$

In other words the chemical potentials at these points are equal. Points $b_{1}$ and $b_{2}$ are called binodal points. The locus of binodal points as a function of $\chi N$ gives the binodal curve which distinguishes a single phase from the phase separated region in the parameter space $\chi N$. In the vicinity of binodal points if the system were to locally phase separate, due to any fluctuations, it will result in an increase in free energy. Thus phase separation is not favoured close to the binodal points. To locate the binodal points in addition to $\left(\partial \Delta f_{\text {mix }} / \partial \phi\right)=0$, the condition $\left(\partial^{2} \Delta f_{\text {mix }} / \partial \phi^{2}\right)>0$ must be satisfied. 
For compositions between the inflection points $s_{1}$ and $s_{2}$ the system is unstable and spontaneously phase separates with respect to any fluctuations in composition. The phase separation process of this sort is known as spinodal decomposition and the corresponding points which act as a boundary encompassing this portion of the free energy curve are called spinodal points. In this region, any fluctuation in composition lowers the free energy and so the system phase separates spontaneously. In the region between the $b$ s and ss, small fluctuations lead to an increase in free energy whereas large fluctuations lead to a decrease in free energy. Hence this region is called metastable. The phase separation process in this region is called binodal decomposition. The phase separation mechanism in binodal decomposition is not spontaneous, unlike spinodal decomposition. At spinodal points we have $\partial^{2} \Delta f_{\text {mix }} / \partial \phi^{2}=0$. The locus of spinodal points as a function of $\chi N$ gives us the spinodal curve. The binodal curve separates the single phase from the phase separated region. The region between the binodal and spinodal curve represents the metastable region. Above the spinodal curve the system is unstable and phase separates spontaneously.

The global free energy or the free energy functional is obtained by integrating the local free energy given by Eq. (6.13) over the entire system volume. This quantity describes the thermodynamic state of the system correctly independent of whether the system is homogeneous or inhomogeneous, unlike the local free energy which always works under homogeneous mixture conditions. The most popular form of the free energy functional to study phase separation in polymer blends is the Flory-Huggins-de Gennes (FHdG) free energy [51] formulism written as

$$
\frac{F_{F H d G}}{k_{B} T}=\int d \mathbf{r}\left[f_{\text {mix }}[\phi(\mathbf{r})]+\frac{1}{36 \phi(\mathbf{r})[1-\phi(\mathbf{r})]}|\nabla \phi(\mathbf{r})|^{2}\right],
$$

The first term inside the integral is the local Flory-Huggins free energy given by Eq. (6.13). The second term represents the penalty, due to local fluctuations, in concentration in the random phase approximation limit proposed by de Gennes [51]. Note that this approximation is only valid in the limit of slow spatial variations.

Though the free energy functional given by Eq. (6.15) is the preferred choice, here we consider a slightly simpler version of this free energy which is more generic for phase separating systems. This simplification is justified since the primary focus of this study is to test the applicability of a numerical method to polymer blends rather than replicating the exact behaviour of the system. For 
this purpose we define the local order parameter to be the difference in the local concentrations i.e., $\psi(\mathbf{r})=\phi_{A}(\mathbf{r})-\phi_{B}(\mathbf{r})$, in the incompressible limit. Rewriting the integrand of the FHdG free energy functional in terms of the order parameter $\psi$ yields

$$
\begin{aligned}
\frac{1}{N}\left[\left(\frac{1+\psi}{2}\right) \ln \left(\frac{1+\psi}{2}\right)+\left(\frac{1-\psi}{2}\right) \ln \left(\frac{1-\psi}{2}\right)+\right. & \left.\chi N\left(\frac{1+\psi}{2}\right)\left(\frac{1-\psi}{2}\right)\right] \\
& +\frac{1}{9\left(1-\psi^{2}\right)}|\nabla \psi|^{2}
\end{aligned}
$$

Using logarithmic and binomial expansions (about $\psi \approx 0$ ) and neglecting the higher order terms, which is a valid approximation since $\psi$ is small in the vicinity of a phase transition, Eq. (6.16) can be approximated by

$$
\frac{1}{2 N}\left[\psi^{2}+\frac{1}{6} \psi^{4}+\mathcal{O}\left(\psi^{6}\right)+\text { constant }\right]+\frac{\chi}{4}-\frac{\chi}{4} \psi^{2}+\frac{1}{9}|\nabla \psi|^{2}
$$

where "constant" represents terms not involving $\psi$. These terms only lower or raise the free energy, but do not affect the position of any maxima or minima. Rearranging the terms in increasing powers of $\psi$ and ignoring the constant terms, we find that the FHdG free energy functional is equivalent to the the Ginzburg-Landau free energy functional

$$
\frac{F_{G L}}{k_{B} T}=\int d \mathbf{r}\left[-\frac{\varepsilon}{2} \psi^{2}+\frac{u}{4} \psi^{4}+\frac{K}{2}|\nabla \psi|^{2}\right] .
$$

The parameters in Eq. (6.18) can be chosen such that they can be mapped to the parameters in Eq. (6.17). Hence this choice for the free energy functional (the Ginzburg-Landau form) is a suitable choice for polymer blends. Another important point to note is the resemblance of Eq. (6.18) to the Cahn-Hilliard (CH) model $[159,160]$ that is employed to study phase separation in binary alloys. In the $\mathrm{CH}$-model the local free energy is assumed to be a function of order parameter and gradients of the order parameter. These gradients of order parameter account for the cost of forming an interface between the dissimilar species.

\subsection{Introduction to SPH}

In $\mathrm{SPH}$, continuum equations that govern the system evolution are solved by approximating the fluid as a collection of particles. Thus it combines features of both continuum and particle methods. In the SPH method, the fluid under consideration is divided into a number of fluid volumes. Each fluid volume is referred to as a particle. Each of these particles possess physical properties such 
as mass, density, velocity, volume, etc. The dynamical evolution of the system is followed by solving the equations of motion, governed by the hydrodynamics of the system. Physical properties that are required to solve these equations of motion are calculated by obtaining a smoothed average of physical properties over all the particles in the system. This method was initially developed to address astrophysical problems but soon found applications in diverse fields ranging from fluid dynamics, mechanics of solids to animations, to name a few [161]. A comprehensive review on $\mathrm{SPH}$ is provided in references [161,145]. In this section we give a brief overview of some of the fundamental concepts in SPH.

The basic idea in SPH is to evaluate the value of a field variable at a particular point in space by calculating a weighted average of the same physical variable from the surrounding particles in the neighbourhood. Ideally, the value of a physical quantity $A$ at point $\mathbf{r}$ is given by

$$
A(\mathbf{r})=\int_{V} A\left(\mathbf{r}^{\prime}\right) \delta\left(\mathbf{r}-\mathbf{r}^{\prime}\right) d \mathbf{r}^{\prime},
$$

where integration is performed over the total volume of the system $V$ and $\delta$ is the Dirac-delta function.

To numerically compute the value of the variable $A(\mathbf{r})$, the Dirac-delta function in Eq. (6.19) is replaced by a kernel $W\left(\mathbf{r}-\mathbf{r}^{\prime}, h\right)$ which acts as a smoothing function over all the particles in volume $V$. Here $h$ is referred to as the smoothing length which effectively controls the weighted contribution to the value of the variable from the distribution of particles that are within the volume of the system. The kernel should satisfy the following conditions:

- The normalization condition: $\int_{V} W\left(\mathbf{r}-\mathbf{r}^{\prime}, h\right) d \mathbf{r}^{\prime}=1$.

- In the limit $h \rightarrow 0$, the kernel $W$ should behave as the Dirac-delta function i.e.,

$$
\lim _{h \rightarrow 0} W\left(\mathbf{r}-\mathbf{r}^{\prime}, h\right) \rightarrow \delta\left(\mathbf{r}-\mathbf{r}^{\prime}\right)
$$

Replacing the $\delta$ function in Eq. (6.19) with $W$ yields

$$
A(\mathbf{r})=\int_{V} A\left(\mathbf{r}^{\prime}\right) W\left(\mathbf{r}-\mathbf{r}^{\prime}, h\right) d \mathbf{r}^{\prime} .
$$

The continuous integration over the whole volume in Eq. (6.20) can be approximated by the summation over all the particles by dividing the system into a number of particles. This is known as the particle approximation [162]. Each 
particle has a mass, volume and density. Hence rewriting Eq. (6.20)

$$
A(\mathbf{r})=\sum_{j=1}^{N} A\left(\mathbf{r}_{j}\right) W\left(\mathbf{r}-\mathbf{r}_{j}, h\right) \Delta \mathbf{r}_{j},
$$

where the index $j$ runs over all the particles in the system, $N$ and $\Delta \mathbf{r}_{j}=m_{j} / \rho_{j}$ is the volume of particle $j$ with mass $m_{j}$ and density $\rho_{j}$. Thus the value of the physical variable $A(\mathbf{r})$ is a smoothed weighted average over all the particles $j$. Therefore,

$$
A(\mathbf{r})=\sum_{j=1}^{N} \frac{m_{j}}{\rho_{j}} A\left(\mathbf{r}_{j}\right) W\left(\mathbf{r}-\mathbf{r}_{j}, h\right) .
$$

While the summation in Eq. (6.21) is over all the particles in the system, in reality, only a small number of particles that are in the vicinity of the central particle contribute to the sum with negligible contribution from the rest. This aspect can be utilized to narrow down the number of contributing particles to a small influence area (volume in 3D) around each particle and hence reducing the computational effort. This region of influence is known as the support domain. The area under the support domain can be controlled by varying the smoothing length $h$. A physical interpretation of the above statement can be provided in the following way. The smoothing length $h$ defines the decay of $W$ as a function of distance from the central particle. If $h$ is small then particles that are close to the central particle contribute the most to the sum. Therefore the summation in Eq. (6.21) can be reduced to particles only within the support domain.

One of the major advantages of using the SPH method is that spatial derivatives can be calculated without the aid of a discrete grid. In SPH, the gradient of function $A(\mathbf{r})$ is given by

$$
\nabla_{\mathbf{r}} A(\mathbf{r})=\sum_{j} \frac{m_{j}}{\rho_{j}} A\left(\mathbf{r}_{j}\right) \nabla_{\mathbf{r}} W\left(\mathbf{r}-\mathbf{r}_{j}, h\right)
$$

The above equation does not yield correct results since it gives a non-zero contribution even if the function $A$ is constant. This leads to violation of the conservation of linear and angular momentum. To rectify this, the following identity is used,

$$
\rho \nabla A=\nabla(\rho A)-A \nabla \rho,
$$

where $\rho$ is the density of the particle. Eq. (6.23) not only conserves linear and angular momentums but also helps to achieve higher orders of accuracy [163]. 
Using Eq. (6.23), Eq. (6.22) can be rewritten as

$$
\nabla_{\mathbf{r}} A(\mathbf{r})=\sum_{j} \frac{m\left(\mathbf{r}_{j}\right)}{\rho(\mathbf{r})}\left[A\left(\mathbf{r}_{j}\right)-A(\mathbf{r})\right] \nabla_{\mathbf{r}} W\left(\mathbf{r}-\mathbf{r}_{j}, h\right) .
$$

The density of a particle can be calculated in two ways. Using the definition in Eq. (6.21) the density of particle $i$ is

$$
\rho_{i}=\sum_{j} m_{j} W\left(\mathbf{r}_{i}-\mathbf{r}_{j}, h\right)
$$

Alternatively one can use the continuity equation:

$$
\frac{d \rho}{d t}+\rho \nabla \cdot \mathbf{v}=0
$$

From Eqs. (6.24) and (6.26), for any particle $i$, we have

$$
\frac{d \rho_{i}}{d t}=\sum_{j} m_{j}\left(\mathbf{v}_{i}-\mathbf{v}_{j}\right) \cdot \nabla_{i} W\left(\mathbf{r}_{i}-\mathbf{r}_{j}, h\right),
$$

where $\mathbf{v}_{i}$ and $\mathbf{v}_{j}$ are the velocities of the particles $i$ and $j$, respectively. In general Eq. (6.25) is widely used to calculate the density but shown to have problems at free surfaces. It leads to oscillations in density of particles at free surfaces which in turn causes fluctuations in pressure. This leads to unphysical behaviour of surface particles. On the other hand using Eq. (6.27) to compute densities is found to be much more stable at handling free surfaces. In Eq. (6.27), when particles approach each other their relative velocity becomes negative thus increasing the density of that particle. This leads to an increase in pressure on the particles and hence at subsequent times these particles are pushed apart from each other to stabilize the density or pressure. Note, that Eqs. (6.25) and (6.27) are equivalent since formally differentiating Eq. (6.25) gives Eq. (6.27).

The above discussion only provides a brief introduction to the SPH methodology providing the grounds by which we can apply it to our polymer blends. We do not indulge in a serious discussion on the other aspects involving $\mathrm{SPH}$ as they are irrelevant to our study here. Rather than getting into the specific details about the method, our focus here is to consider whether SPH can be applied to phase separating polymer systems. 


\subsection{Application of SPH to Polymer Blends}

The SPH method has been successfully applied to polymer blends by Okuzono [164]. Using the two-fluid model [165], he demonstrated the applicability of SPH to study phase separation in polymer blends. This study was restricted to fluids with periodic boundary conditions. However the real strength of the SPH method lies in its ability to model complicated bounding geometries or free surfaces. Here we attempt to extend Okuzono's work to polymer blends with bounding surfaces (which the blend cannot penetrate) and to free surfaces. Essentially the underlying theory remains the same as that used by Okuzono. We provide a brief overview of the necessary background and also derive the equations that need to be solved.

Consider a binary polymer mixture consisting of polymers $A$ and $B$. The system domain is divided into a number of equi-volume fluid elements or quasi-particles. For simplicity we shall just refer to these fluid elements as particles. Let us denote the number of $A$ particles by $N_{A}$ and the number of $B$ particles by $N_{B}$. Initially, each of these particles consist both $A$ and $B$ polymer densities. As a convention, we use indices $i, j, \cdots$ to indicate $A$ particles, $n, m, \cdots$ for $B$ particles, and $\alpha, \beta, \cdots$ for any particle. Using the notation described above, the $A$ and $B$ densities for any particle $\alpha$ are given by

$$
\begin{aligned}
\rho_{A}\left(\mathbf{r}_{\alpha}\right) & =\sum_{j=1}^{N_{A}} m_{A} W\left(\mathbf{r}_{\alpha}-\mathbf{r}_{j}, h\right), \\
\rho_{B}\left(\mathbf{r}_{\alpha}\right) & =\sum_{n=1}^{N_{B}} m_{B} W\left(\mathbf{r}_{\alpha}-\mathbf{r}_{n}, h\right),
\end{aligned}
$$

where $m_{A}=M_{A} / N_{A}$ and $m_{B}=M_{B} / N_{B}$ are masses of individual $A$ and $B$ particles, respectively; $M_{A}$ and $M_{B}$ are the total mass of $A$ and $B$ fluid in the system. Thus, the total density of particle $\alpha$ is $\rho\left(\mathbf{r}_{\alpha}\right)=\rho\left(\mathbf{r}_{\alpha A}\right)+\rho\left(\mathbf{r}_{\alpha B}\right)$. During the course of the simulation, irrespective of its $A$ and $B$ compositions (densities), a particle's identity does not change. The governing equations of motion act such that if an $A$ particle is in a $B$ rich region, which implies $\rho_{A}<\rho_{B}$, it moves away from its current location towards an $A$ rich region and vice-versa. Eventually the system phase separates on the macroscale.

The smoothing kernel was chosen to be the Gaussian, due to its mathematical 
simplicity in implemention, given by

$$
W\left(\mathbf{r}_{i}-\mathbf{r}_{j}\right)=\left(\frac{1}{\pi h^{2}}\right)^{d / 2} e^{-\left(\mathbf{r}_{i}-\mathbf{r}_{j}\right)^{2} / h^{2}},
$$

where $d$ is the dimensionality of the system. We derive the equations of motion using the Lagrangian formulism. The Lagrangian for the system under consideration is:

$$
\mathcal{L}=\sum_{\beta} m_{\beta}\left[\frac{1}{2} \dot{\mathbf{r}}_{\beta}^{2}-f\left(\rho_{\beta}, \phi_{A \beta}, \phi_{B \beta}\right)\right],
$$

where $f\left(\rho_{\beta}, \phi_{A \beta}, \phi_{B \beta}\right)$ is the free energy density per unit mass and $\phi_{A \beta}=\rho_{A \beta} / \rho_{\beta}$ and $\phi_{B \beta}=\rho_{B \beta} / \rho_{\beta}$ are the mass fractions of $A$ and $B$ fluids, respectively.

In case of polymer fluids, additional forces arise from the viscoelastic effects due to stress exerted on their conformations due to motion. We ignore this effect in our simulations. For binary polymer mixtures, associated with this is the dissipation that originates from relative motion and velocity gradients between dissimilar species. This effect is incorporated into our model via the dissipation function [165]

$$
\mathcal{R}=\frac{1}{2} \sum_{\beta} \frac{m_{\beta} \zeta_{\beta}}{\rho_{\beta}}\left(\mathbf{v}_{A}-\mathbf{v}_{B}\right)_{\beta}^{2}+\sum_{\beta} \frac{m_{\beta} \eta}{\rho_{\beta}} \mathbf{D}_{\beta}: \mathbf{D}_{\beta},
$$

where $\zeta_{\beta}, \mathbf{v}_{A \beta}\left(\left.\equiv \mathbf{v}_{A}\right|_{\beta}\right)$, and $\mathbf{v}_{B \beta}\left(\left.\equiv \mathbf{v}_{B}\right|_{\beta}\right)$ are the friction coefficient, $A$ fluid velocity, and $B$ fluid velocity of particle $\beta$. We assumed the viscosity of both fluids to be equal to $\eta$. $\mathbf{D}_{\beta}$ is the velocity gradient tensor defined as

$$
\mathbf{D}_{\beta}=\frac{1}{2}\left[(\nabla \mathbf{v})_{\beta}+(\nabla \mathbf{v})_{\beta}^{T}\right]
$$

where $(\nabla \mathbf{v})_{\beta}$ is calculated using the definition

$$
(\nabla \mathbf{v})_{\beta}=\sum_{\gamma} \frac{m_{\gamma}}{\rho_{\beta}}\left(\mathbf{v}_{\gamma}-\mathbf{v}_{\beta}\right) \nabla_{\beta} W_{\beta \gamma}
$$

where

$$
\mathbf{v}_{\alpha}=\sum_{\beta} \frac{m_{\beta}}{\rho_{\beta}} \mathbf{v}_{\beta} W_{\alpha \beta} .
$$

is the velocity of any particle $\alpha$. 
Now, the equation of motion for any particle $\alpha$ is

$$
\frac{d}{d t} \frac{\partial \mathcal{L}}{\partial \dot{\mathbf{r}}_{\alpha}}-\frac{\partial \mathcal{L}}{\partial \mathbf{r}_{\alpha}}+\frac{\partial \mathcal{R}}{\partial \dot{\mathbf{r}}_{\alpha}}=0
$$

Inserting Eqs. (6.31) and (6.32) into Eq. (6.36), and after some algebra we finally arrive at

$$
\ddot{\mathbf{r}}_{\alpha}=\sum_{\beta}\left(\frac{\underline{\underline{\varrho}}_{\alpha \beta}}{\rho_{\alpha}^{2}}+\frac{\underline{\mathfrak{\varrho}}_{\beta \alpha}}{\rho_{\beta}^{2}}\right) \cdot \nabla_{\alpha} W_{\alpha \beta} \mp \mathfrak{P} \frac{\zeta_{\alpha}}{c_{\alpha}}\left(\mathbf{v}_{A}-\mathbf{v}_{B}\right)_{\alpha} .
$$

To obtain a dimensionless form, the above equation was rescaled with respect to $l_{0}, u_{0}$ and $\rho_{0}$ which are units of length, velocity, and density, respectively. The first term in Eq. (6.37) involves the stress tensor $\propto$ and describes the interaction between the particles. In the second term in Eq. (6.37), $\mathfrak{P}$ is a system parameter, $\zeta_{\alpha}=\left(\rho_{A} \rho_{B} / \rho^{2}\right)_{\alpha}$ is the friction coefficient and $c_{\alpha}=\rho_{A}$ or $\rho_{B}$ depending on whether $\alpha=A$ or $B$. The sign of the second term is minus if the particle $\alpha$ is of type $A$ and plus if it is of type $B$.

The stress tensor $\underline{=}_{\alpha \beta}$ is written as

$$
\underline{\underline{\varrho}}_{\alpha \beta} \equiv-\left(p_{\alpha}+\mu_{\alpha \beta}\right) \underline{\underline{I}}+2 \mathfrak{R}^{-1} \mathbf{D}_{\alpha}
$$

where $\underline{\underline{I}}$ is the unit tensor, $\mu_{\alpha \beta}$ is the chemical potential between particles $\alpha$ and $\beta$, $\mathbf{D}_{\alpha}$ the velocity gradient tensor, and $\mathfrak{R}=\rho_{0} u_{0} l_{0} / \eta$ is a dimensionless parameter. We now define each entity in Eq. (6.38) and explain their significance.

Although the fluid mixture was assumed to be incompressible, this is not possible to realize in simulations as there is always finite compressibility required to allow particle motion. Real fluids also have finite compressibility. The state of finite compressibility is included via the equation of state:

$$
p_{\alpha}=p_{0}\left[\left(\frac{\rho_{\alpha}}{\bar{\rho}}\right)^{\gamma}-1\right]
$$

where $p_{0}$ is a positive constant and $\bar{\rho}$ is the average density of the system. If the density of any particle $\alpha$ deviates from the system average then it results in a change in pressure at that particle/point. Globally, this drives the system towards a constant density or zero-pressure state. If $\rho_{\alpha}>\bar{\rho}$ then a positive pressure is exerted on the particle which will push the particles farther apart from each other and vice-versa. Obviously the magnitude of pressure depends on the right set of parameters for $p_{0}$ and $\gamma$ which are system dependent. In our simulations we use Okuzono's values for these parameters, $p_{0}=2.0$ and $\gamma=2$. 
The chemical potential is responsible for driving the system towards phase separation. Note, if the system is in the binodal-spinodal region of the phase diagram, fluctuations in the order parameter can drive the system towards phase separation. The ultimate stable phase is the one where the chemical potential exchange between the two phases is equal. Due to its simplicity and relevance to small molecular systems we use the Ginzburg-Landau form of free energy in Eq. (6.18) to compute the free energy

$$
\begin{aligned}
& \mu_{\alpha}=\frac{\partial F_{G}}{\partial \phi}, \\
& \mu_{\alpha}=-\epsilon \phi_{\alpha}+u \phi_{\alpha}^{3}-K\left(\nabla^{2} \phi\right)_{\alpha},
\end{aligned}
$$

where $\phi_{\alpha}=\left(\rho_{A}-\rho_{B}\right)_{\alpha} / \rho_{\alpha}$ is the local order parameter for the particle $\alpha$ and $\epsilon, u$ and $K$ are constants. The first and second terms are readily available from Eqs. (6.28) and (6.29). Now considering the third term in Eq. (6.41), we have

$$
\nabla^{2} \phi_{\alpha}= \begin{cases}\nabla^{2}\left(\left(\rho_{A}-\rho_{B}\right) / \rho\right)_{\alpha} & \text { if } \alpha=A \\ \nabla^{2}\left(\left(\rho_{B}-\rho_{A}\right) / \rho\right)_{\alpha} & \text { if } \alpha=B\end{cases}
$$

The sign convention is taken care of in the final definition for chemical potential. Assuming that the incompressibility condition approximately holds, we are left with

$$
\nabla^{2} \phi_{\alpha} \approx \begin{cases}2\left(\nabla^{2} \rho_{A}\right)_{\alpha} / \rho_{\alpha} & \text { if } \alpha=A \\ 2\left(\nabla^{2} \rho_{B}\right)_{\alpha} / \rho_{\alpha} & \text { if } \alpha=B\end{cases}
$$

Using Eq. (6.23), the Eq. (6.43) can be rewritten as

$$
\begin{aligned}
& \left(\nabla^{2} \rho_{A}\right)_{i}=\sum_{j} \frac{m_{A}}{\rho_{A i}}\left[\left(\nabla \rho_{A}\right)_{j}-\left(\nabla \rho_{A}\right)_{i}\right] \cdot \nabla_{i} W_{i j} \quad \text { if } \alpha=A, \\
& \left(\nabla^{2} \rho_{B}\right)_{n}=\sum_{m} \frac{m_{B}}{\rho_{B n}}\left[\left(\nabla \rho_{B}\right)_{m}-\left(\nabla \rho_{B}\right)_{n}\right] \cdot \nabla_{n} W_{n m} \quad \text { if } \alpha=B .
\end{aligned}
$$

Keeping the above convention in mind, we re-define the order parameter as

$$
\hat{\phi}_{\alpha}= \begin{cases}\left(\left(\rho_{A}-\rho_{B}\right) / \rho\right)_{\alpha} & \text { if } \alpha=A \\ \left(\left(\rho_{B}-\rho_{A}\right) / \rho\right)_{\alpha} & \text { if } \alpha=B\end{cases}
$$

Therefore, the chemical potential takes the following form

$$
\begin{aligned}
\hat{\mu}_{\alpha \beta} & =\epsilon_{\alpha \beta} \hat{\mu}_{\alpha}, \\
& =\epsilon_{\alpha \beta}\left(-\varepsilon \hat{\phi}_{\alpha}+u \hat{\phi}_{\alpha}^{3}-K \nabla^{2} \hat{\phi}_{\alpha}\right) .
\end{aligned}
$$


where $\epsilon_{\alpha \beta}=1$ if $\alpha$ and $\beta$ are both of same type and -1 otherwise.

Having defined all the entities in Eq. (6.37), the next step is determine the time evolution of the system. In the next section, we introduce some tools to achieve this.

\subsection{Implementation}

\subsubsection{Initial Configuration}

All the simulations presented in this chapter are of two-dimensional systems. The system consists of two types of particles, $A$ and $B$. The system domain size is denoted as $L_{x} \times L_{y}$ where $L_{x}$ and $L_{y}$ are its dimensions in the $x$ and $y$ directions, respectively. In all our simulations, the initial configuration has $L_{x}=L_{y}=L$. All systems contain $N=2 L_{x} L_{y}$ particles, which gives a fluid particle volume (actually area since we are in two dimensions) of 1 . The fluid particle size can be varied (by changing $N$ ) and should be made sufficiently small so that the simulation results are independent of particle size. We use a smoothing length of 1.2 times the fluid particle size.

The initial configuration of the system was generated using one of the following two methods. First we shall discuss the case of symmetric polymer blends i.e., $N_{A}=N_{B}$. All the $A$ particles are placed on a square lattice of lattice constant 1.0. Another similar lattice was created for $B$ particles. These two lattices were then superimposed on each other and the $B$ lattice was displaced by $(0.5,0.5)$ with respect to the $A$ lattice.

Clearly, the above described method is not applicable for asymmetric polymer blends. As an example consider a system where $N_{A}<N_{B}$. In this case, particles belonging to the minority type $(A)$ are distributed randomly on the lattice, first. Then the remaining vacant lattice sites are filled with the particles of majority type $(B)$. Note in both methods, the initial particle coordinates lie between $(0,0)$, $\left(L_{x}, L_{y}\right)$.

\subsubsection{Time Integration}

The time evolution of the system was achieved by discretizing the equation of motion, Eq. (6.37), in time. In our simulations we use the Improved Euler method for this purpose. This method involves two steps: a predictor step and a corrector step. In the predictor step, particle velocities and positions are calculated at half 
timesteps as given by

$$
\begin{aligned}
& v_{n+1 / 2}=v_{n}+\frac{\Delta t}{2} a_{n-1 / 2}, \\
& x_{n+1 / 2}=x_{n}+\frac{\Delta t}{2} v_{n} .
\end{aligned}
$$

Here $v, x$ and $a$ indicate velocity, position and acceleration of a particle. $n(\geq 0)$ is the timestep index and $\Delta t$ is the timestep size. At time $t=0, a_{-1 / 2}=0$. Thus obtained particle positions and velocities are used to compute the forces at half timesteps, $a_{n+1 / 2}$. These forces were then used in the corrector step to compute particle positions and velocities at the full time step:

$$
\begin{aligned}
v_{n+1} & =v_{n}+\Delta t a_{n+1 / 2}, \\
v^{*} & =v_{n}+\frac{\Delta t}{2} a_{n+1 / 2} \\
x_{n+1} & =x_{n}+\Delta t v^{*} .
\end{aligned}
$$

\subsubsection{Linked List}

As described earlier, in evaluating any physical quantity we only need particles that are within the support domain around each particle. For a system of $N$ particles there exist $N^{2}$ number of pair-wise interactions, which is a large number even for a system of $10^{3}$ particles. Out of these $N^{2}$ interactions only a small fraction have significant contribution towards evaluated physical quantities. By limiting the pair-wise calculations to the support domain the insignificant pair calculations are avoided thus greatly reducing the required computational effort. In our simulations, we use the linked-list method [162] for this purpose.

In the linked-list method, the system domain is divided into $M$ cells of equal size. The dimensions of the cell are $d m_{x}$ and $d m_{y}$ in $x$ and $y$ directions, respectively. The condition $d m_{x}, d m_{y} \geq r_{\text {cut }}$ has been imposed on the cell dimensions. This was done to ensure that for any particle $i$, all other particles that belong to its support domain reside in its immediate neighbouring cells. In this manner we can restrict neighbour searching to the least possible number of cells (8 in 2D). The linked list implementation is illustrated in Fig. 6.2. For any particle $i$, all the other particles that contribute to its physical quantities are always within its support domain. We use a $r_{\text {cut }}$ of $3 h$. 


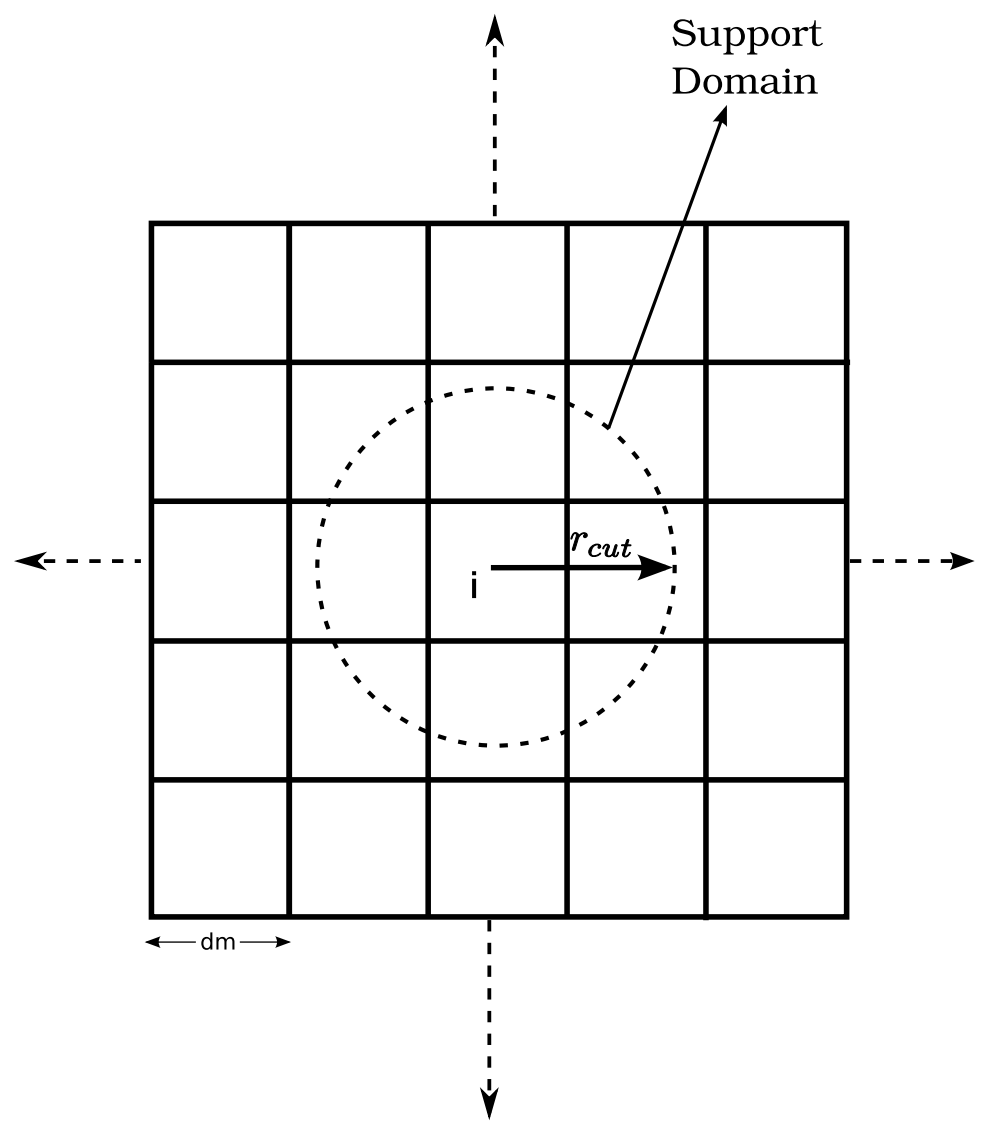

Figure 6.2: A schematic diagram showing the linked-list mechanism for a small part of the system. The area under the circle represents the support domain for the particle $i$. In this particular case $d m_{x}=d m_{y}=d m$. The cell dimension always satisfies the following condition $d m \geq r_{\text {cut }}$, where $r_{c u t}$ is the cut-off radius for interaction. The dotted arrows indicate presence of fluid beyond the boundaries shown in the figure.

\subsection{Results}

In all our simulations, we set $u=1.0$ and $K=0.5$ in Eq. (6.41). The timestep size was fixed to $\Delta t=0.005$. This choice for $\Delta t$ was found to be adequate for our purposes and did not cause large fluctuations in particle densities. Most of the time $\rho \approx 1.0 \pm 0.07$ which is small enough so as to not influence the phase separation process. This observation also confirms that the choice of parameters $p_{0}=2.0$ and $\gamma=2.0$ in Eq. (6.39) are adequate for our purposes.

To realize a disordered state, the initial configuration of the blends was simulated for 100 time units with $\varepsilon=-0.5$. After this period, the $\varepsilon$ was changed to 0.5 and the subsequent time evolution of the phase separation process was recorded. The phase separation process was monitored with the help of the local 
order parameter

$$
\phi_{\alpha}=\phi_{A \alpha}-\phi_{B \alpha}=\frac{\rho_{A \alpha}-\rho_{B \alpha}}{\rho_{\alpha}},
$$

as a function of time $t$, for all particles.

The amount of $A$ and $B$ fluids are conserved at all times. This can be measured using the global order parameter:

$$
\frac{1}{N} \sum_{\alpha}^{N} \phi_{\alpha}=1-2 f
$$

where $f$ is the volume fraction of $A$ fluid in the system. In all our simulations, $\Phi$ was conserved at all times except for small fluctuations induced due to compressibility of the fluid.

\subsubsection{Blend with Periodic Boundary Conditions}

First of all we studied the phase separation of a polymer blend with periodic boundary conditions applied in both directions. The phase separation process was monitored by observing the local order parameter evolution as a function of time $t$. The results for the symmetric polymer blend $f=0.5$ for a $50 \times 50$ system are shown in Fig. 6.3. At $t=0$, the system is in a disordered state and hence the local order parameters of all particles are close to zero. Upon quenching the system starts to phase separate. The homogeneity of the phase separation process, at early times, indicates that the underlying process is spinodal decomposition. At early times ( $t=10$ to 50 ), the smaller droplets of one phase get enveloped by larger droplets of that phase. At later times the domains rich in $A$ and $B$ phases grow in size. Note, that the segregation is relatively weak (the order parameter maximum only reaches 0.75 ) which is in keeping with Ginzburg-Landau model we are using to describe the system's free energy.

Simulations for the asymmetric blend case of $f=0.4$ were also carried out and the results are shown in Fig. 6.4. In this case the minority phase initially forms circular droplets. This behaviour is similar to the $f=0.5$ case. As for the $f=0.5$ case, small droplets coalesce (or are enveloped) with other droplets of the minority phase. In the long time limit these circular droplet increase in size. This is the behaviour commonly observed in asymmetric polymer blends [164] and our results are in qualitative agreement with these findings. In principle, we could continue on these simulations to smaller $f$, however the behaviour described above is typical - for smaller $f$ circular droplets only form. 


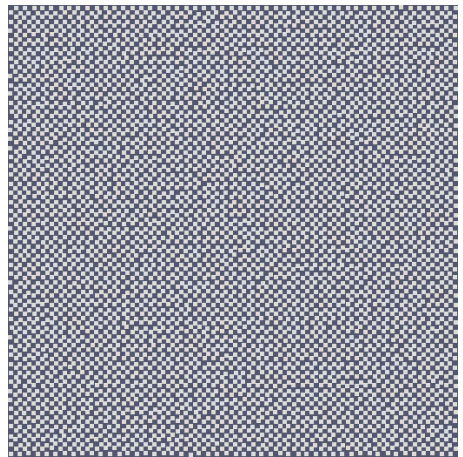

(a) $t=0$

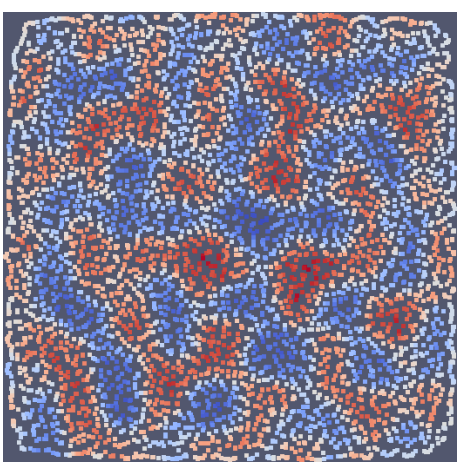

(d) $\quad t=15$

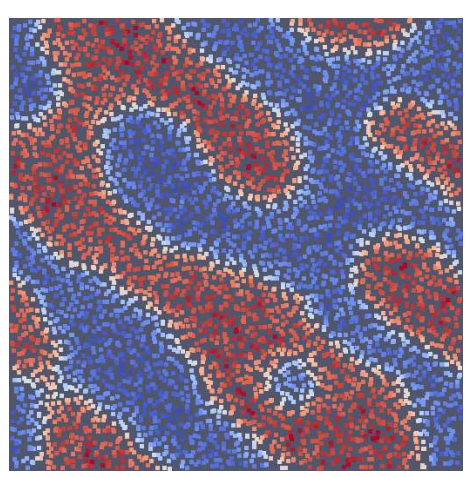

(g) $\quad t=100$

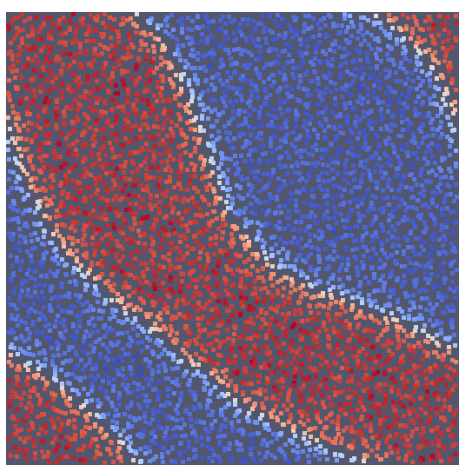

(j) $t=400$

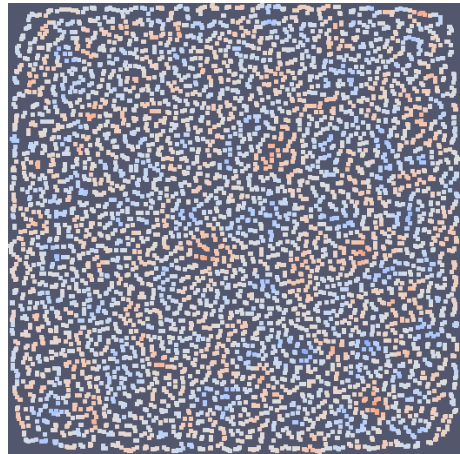

(b) $\quad t=5$

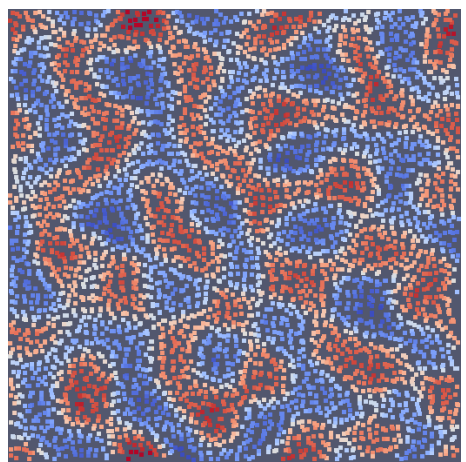

(e) $\quad t=25$

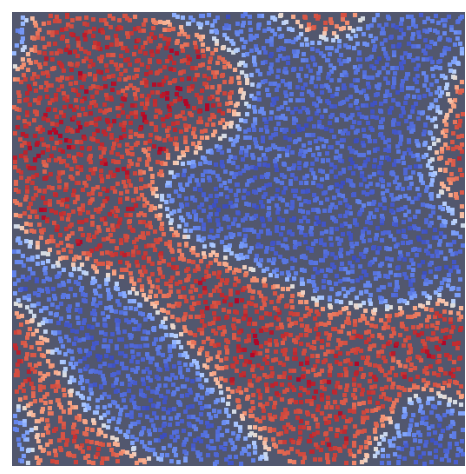

(h) $\quad t=200$

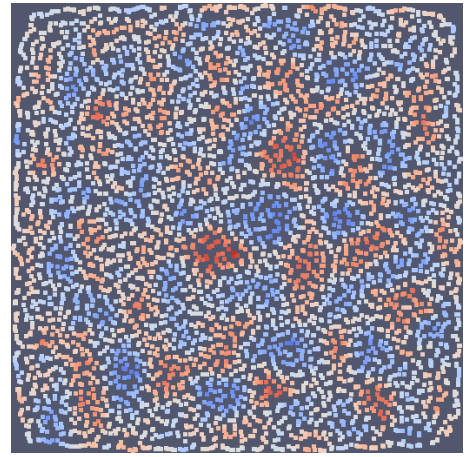

(c) $t=10$

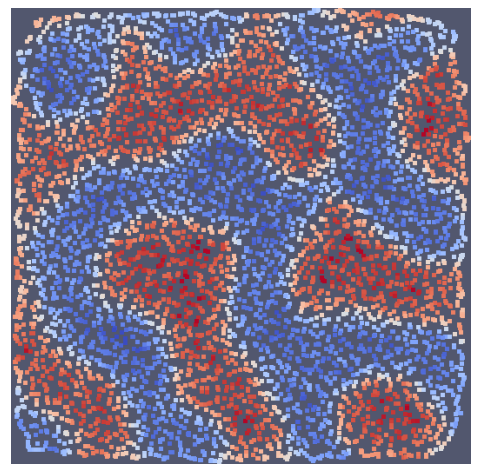

(f) $\quad t=50$

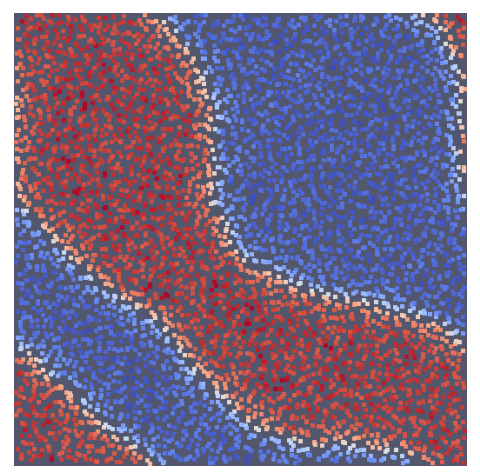

(i) $\quad t=300$
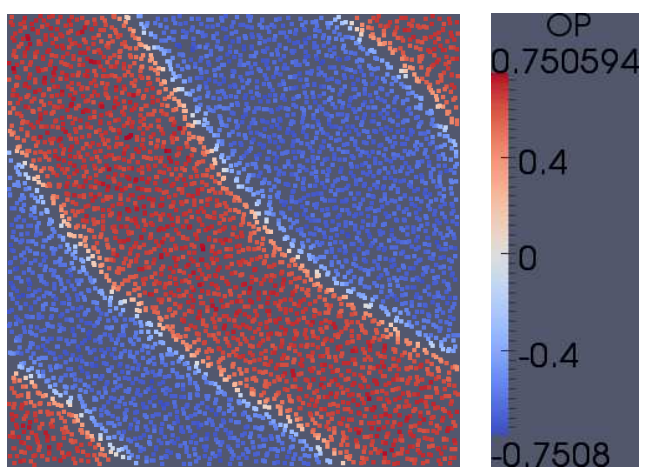

(k) $\quad t=900$

Figure 6.3: The phase separation process for a symmetric polymer blend of size $50 \times 50$ with periodic boundary conditions applied. Plotted is the local order parameter as a function of time $t$. The red colour represents phase $A$ and the blue colour represents the $B$ phase. The degree of phase separation is indicated by the colour scale of the order parameter as shown in the last figure. 


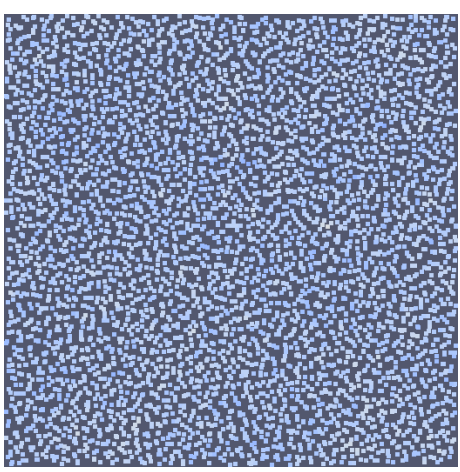

(a) $t=0$

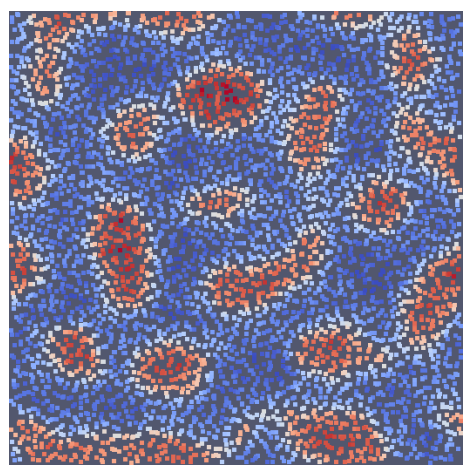

(d) $\quad t=25$

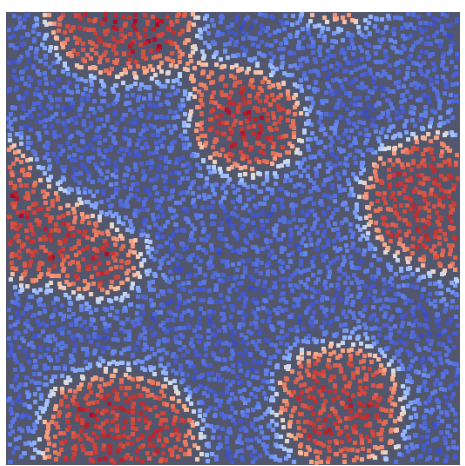

(g) $t=200$

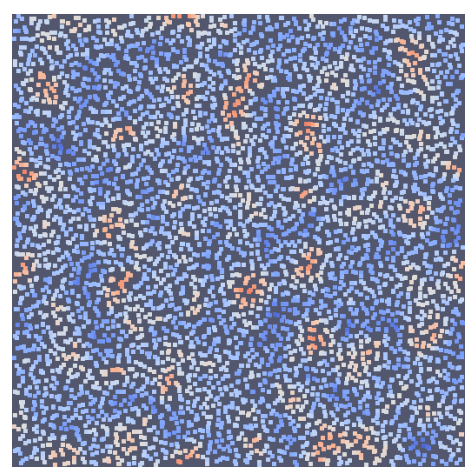

(b) $t=10$

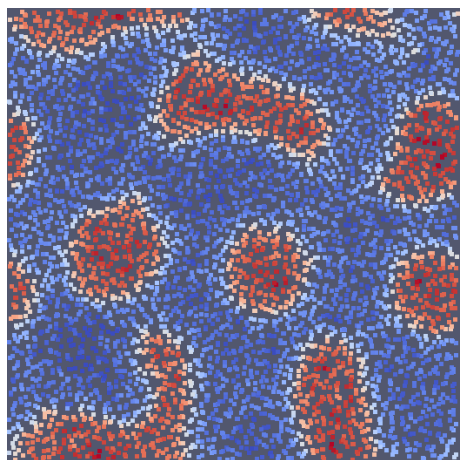

(e) $t=50$

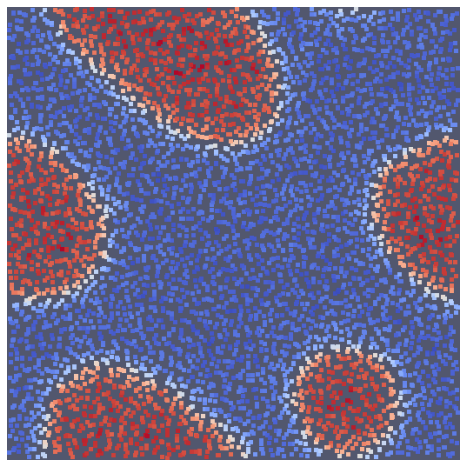

(h) $t=300$

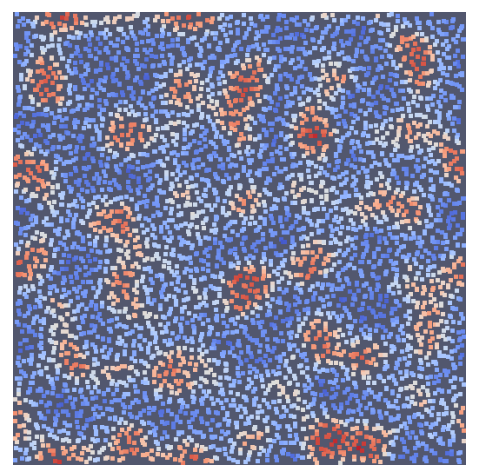

(c) $t=15$

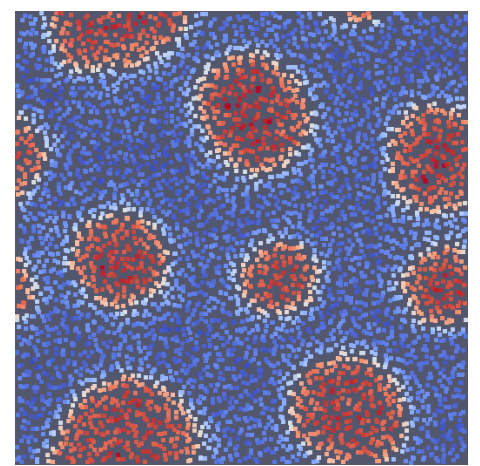

(f) $\quad t=100$

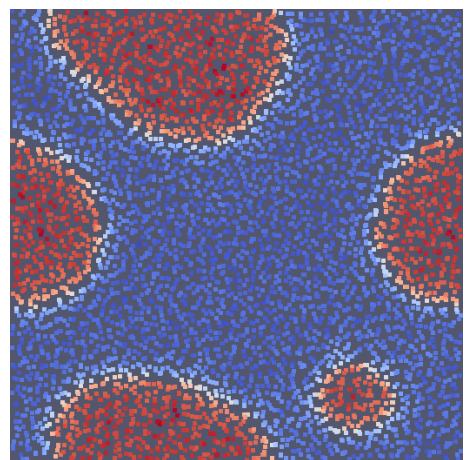

(i) $t=400$
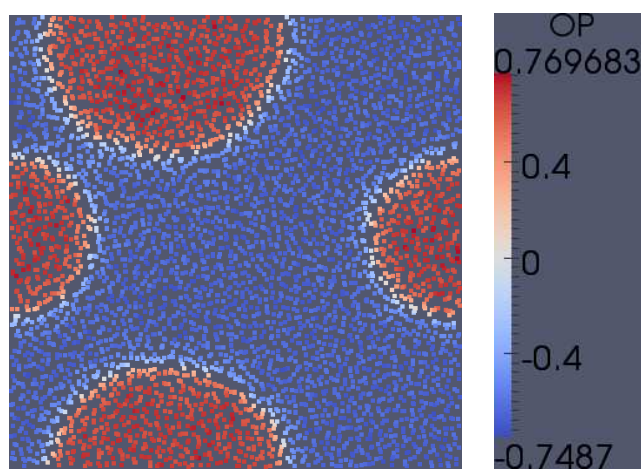

(j) $t=900$

Figure 6.4: The phase separation process for asymmetric polymer blend with $f=0.4$ of size $50 \times 50$ with periodic boundary conditions. The minority phase droplets grow in size with time. 


\subsubsection{Blend between Two Parallel Plates}

A system of practical importance is a polymer blend confined between two parallel substrates. Here we study the phase separation in such systems using the SPH methodology. With the inclusion of substrates, in addition to the fluid-fluid interactions we have fluid-wall interactions.

The presence of impenetrable walls prevents the fluid particles from entering the wall regions. To model this effect, we adopt an LJ-type force (repulsive part only) between the fluid particles and the walls [166, 167]. The form of this boundary force is:

$$
f(y)= \begin{cases}A(l / y)^{S} & \text { if } 0<y<l \\ D(y-b)^{2} & \text { if } l<y<b \\ 0 & \text { otherwise }\end{cases}
$$

where $y$ is the normal distance of the particle from the substrate surface and the parameters

$$
D=A\left(\frac{s+2}{2 b}\right)^{2} ; l=\frac{s b}{s+2} .
$$

The exponent $s$ determines the steepness of the boundary force. For distances smaller than $l$, the boundary force is large and gradually decreases for distances between $l$ and $b$. For distances beyond $b$ from the surface, particles do not experience the wall. In all our simulations with substrates we have set $A=0.1$, $s=8$ and $b=0.5$. This set of parameters are chosen so that no instabilities are caused in the system due to boundary forces. This choice for boundary force also ensures a smooth force field [166]. Note, this force acts normal to the substrate and into the fluid. This boundary force is added to the $y$ component of the equation of motion given by Eq. (6.37).

Now to model the interfacial attraction between the fluid particles and the walls we incorporate a surface energy term into the free energy functional [157]:

$$
\frac{F_{G L}}{k_{B} T}=\int d \mathbf{r}\left[-\varepsilon \phi^{2}+\frac{u}{2} \phi^{4}+\frac{K}{2}|\nabla \phi|^{2}\right]-\frac{F_{\text {surface }}}{k_{B} T} .
$$

where the last term is the surface energy term and has been chosen to have the following form

$$
F_{\text {surface }}=\int d \mathbf{r} \frac{\sigma}{y^{v}} \phi,
$$

where $\sigma$ is the strength of fluid-wall interaction and the exponent $v$ determines 
the interaction range and steepness of the field; $y$ is the normal distance of the particle from the surface, and is always positive. A small value of $v$ means that the field is long-range and the magnitude of the surface energy will be small close to the walls. A large value of $v$ implies that the surface field is short-range and its magnitude is large close to the walls. By adjusting the values of interaction strength $\sigma$ and exponent $v$, the desired behaviour for the fluid-wall interaction can be achieved. Depending on the sign of $\sigma$ the surfaces can be made to prefer either of the species. If a choice of $\sigma$ leads to the surface preferring $A$ particles, by simply changing its sign the substrate preference can be changed to $B$ particles. Note that the repulsive boundary force is not included in the free energy functional as it is independent of the particle (order parameter) and thus will be eliminated from the chemical potential calculation. Therefore, we had to explicitly define the interaction between the fluid particles and the walls.

The surface energy interactions are obtained by completing a functional derivative on the free energy (Eq. 6.57) to obtain the following chemical potential:

$$
\mu\left(\mathbf{r}_{\alpha}\right)=-\varepsilon \phi_{\alpha}+u \phi_{\alpha}^{3}-K\left(\nabla^{2} \phi\right)_{\alpha}+\sigma\left[\frac{1}{r_{y \alpha}^{v}}-\frac{1}{\left(L_{y}-r_{y \alpha}\right)^{v}}\right],
$$

where $r_{y \alpha}$ is the $y$ coordinate of particle $\alpha$. Note, the surface interaction will favour one phase at the lower surface and the other phase at the upper surface since opposite signs precede each term in the chemical potential, Eq. (6.58).

We have carried out simulations over a wide range of $\sigma$ and $v$ values. Here we only report results for those combinations of $\sigma$ and $v$ for which the surface energy term does not dominate the underlying phase separation process. Note while the system is bounded in the $y$ direction, periodic boundary conditions are applied in the $x$ direction to allow for the particle movement.

In Fig. 6.5, we show the phase separation for the case $\sigma=-0.1$ and $v=1.0$ for a symmetric polymer blend. The usual phase separation behaviour was observed everywhere in the system except for regions close to the walls. The lower wall was found to attract the $A$ particles while the upper wall attracted the $B$ particles. This is evident from the phase evolution snapshots. The domains grow parallel to the substrates. The thickness of the $A$ and $B$ phases next to the substrates was found to increase with time. At long times, the system will eventually phase separate into $A$ and $B$ phases macroscopically with $A$ phase in the lower half and $B$ phase in the upper half of the system geometry.

The effect of the short-range nature of the surface potential was studied by 
changing $v=2.0$ for the above system. The domain growth results for this case are shown in Fig. 6.6. As expected the phase behaviour close to the walls was different from the $v=1.0$ case. For example, compare the configurations at $t=100$ in both cases. Due to the short-range nature of $v=2.0$ surface potential, the thickness of the $A$ phase adjacent to the lower wall was smaller than that of $v=1.0$ case at the same instance. It might well be the case for this simulation that the final state is metastable. Although we have run this simulation for an extended time, the large elliptical droplet remains for a long time, even though we would expect lamellae in this case.

Simulations were also carried out for larger values of $\sigma$. The results for the case $\sigma=-0.5$ are shown in Figs. 6.7 and 6.8. Due to a stronger fluid-wall interaction, in the long-range case of $v=1.0$, we observe macroscopic phase separation which was not seen for $\sigma=-0.1$, for the same $v$. For the $v=2.0$ case (Fig. 6.8), the blend forms stripes of $A$ and $B$ phases parallel to the wall.

We have also carried out simulations for positive values of $\sigma$ and its effect on the phase separation was noted. The results for $\sigma=0.1$ and $v=1.0$ are shown in Fig. 6.9. Unlike $\sigma=-0.1$, here the $B$ phase is attracted to the lower wall while the $A$ phase is attracted to the upper wall. This confirms that by changing the sign of the interaction parameter, the alignment of the phases can be changed.

Simulations for the asymmetric blend case of $f=0.4$ were also studied and the results shown in Fig. 6.10. The minority phase was found to form droplets as expected. The effect of the surface interaction on the phase separation process was evident. The lower wall attracts the minority phase which eventually lead to macroscopic phase separation at long times.

It should be noted that the boundary condition here is very simple to implement. The encouraging aspect of this work is that we could simulate much more complicated bounding geometries such as circles or other regular or even irregular geometries. All one needs is to specify the (local) unit normal at the boundary. 


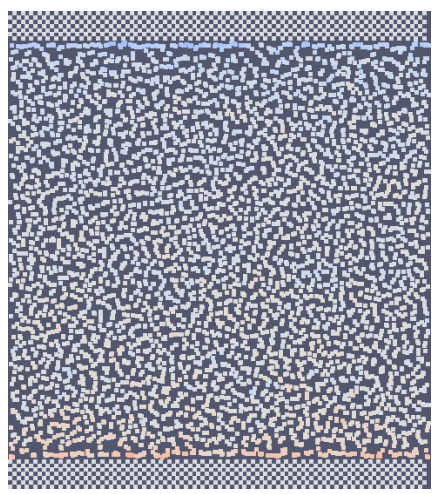

(a) $\quad t=0$

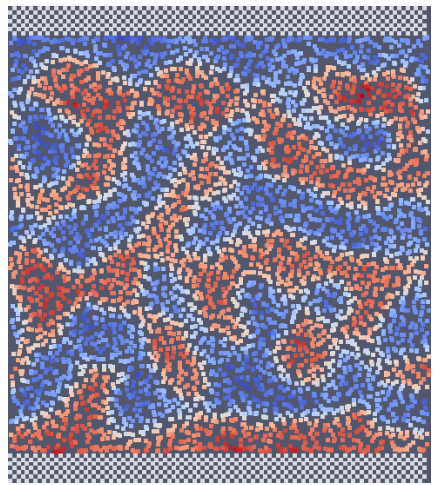

(d) $\quad t=25$

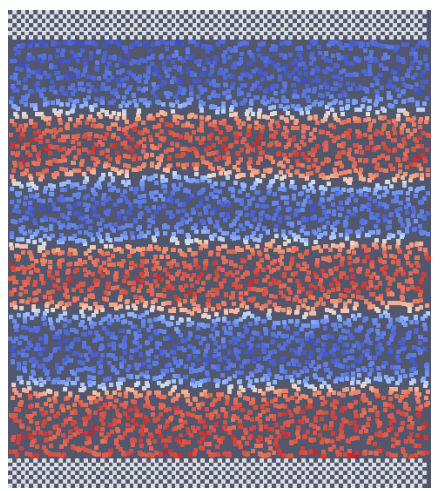

(g) $\quad t=400$

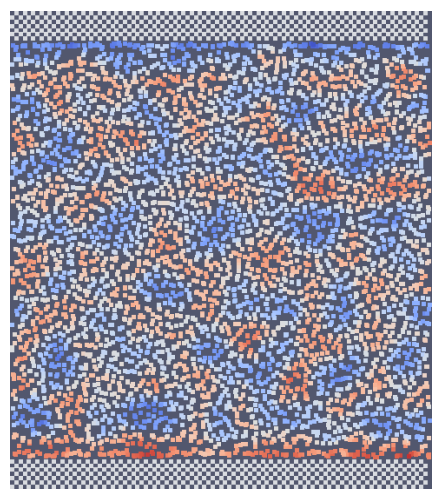

(b) $\quad t=10$

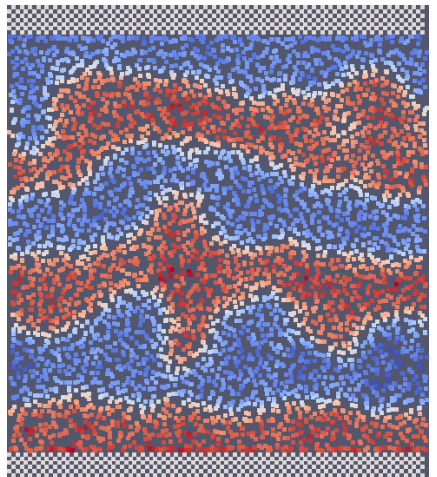

(e) $\quad t=50$

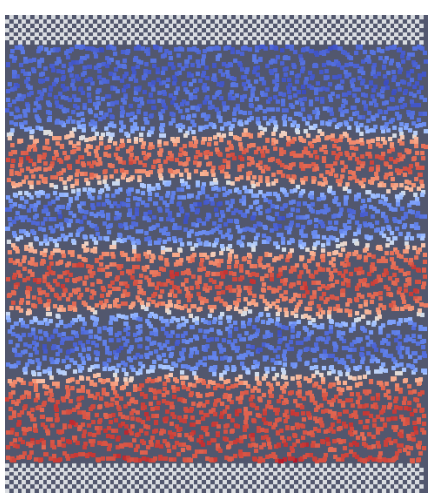

(h) $\quad t=900$

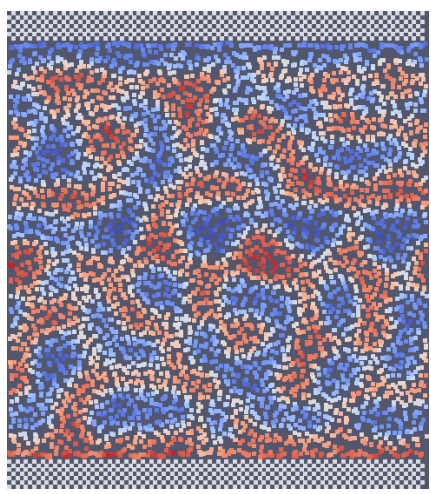

(c) $\quad t=15$

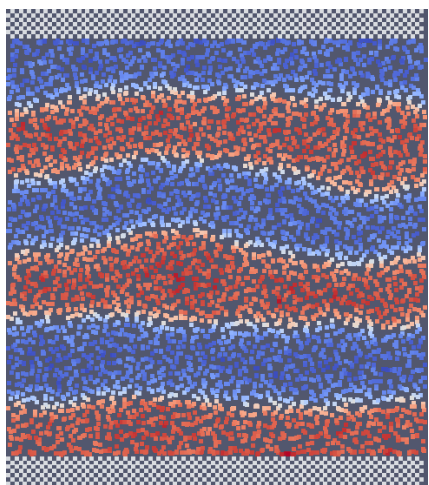

(f) $\quad t=100$

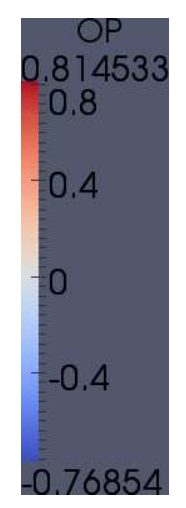

Figure 6.5: Evolution of the spinodal decomposition in the presence of two parallel walls. Shown are the snapshots of the phase evolution for a symmetric $50 \times 50$ polymer blend system with $\sigma=-0.1$ and $v=1.0$. 


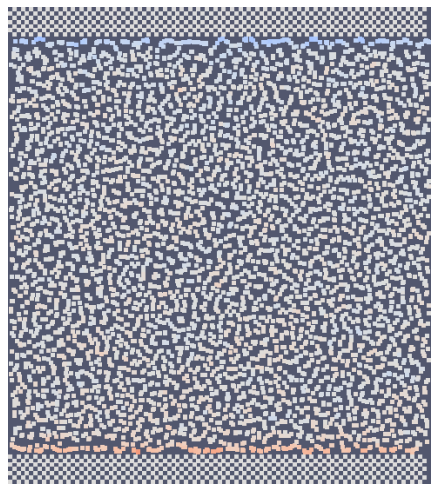

(a) $t=0$

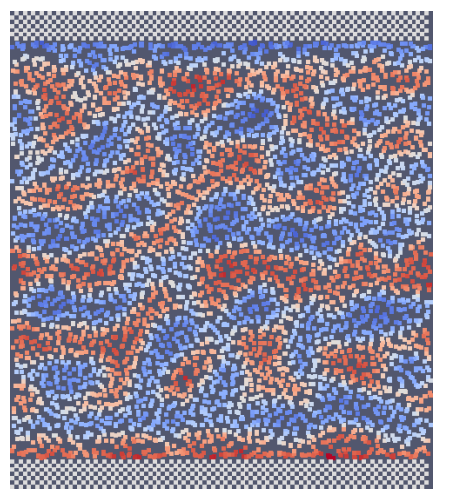

(d) $\quad t=15$

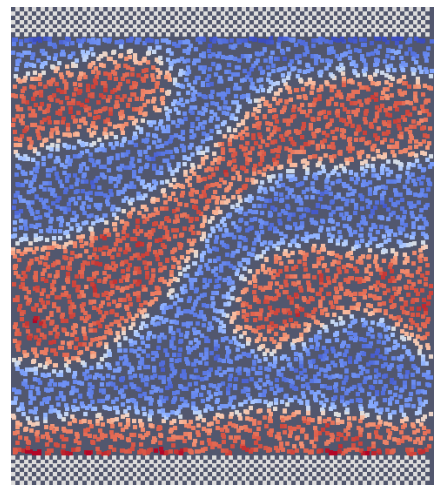

(g) $\quad t=100$

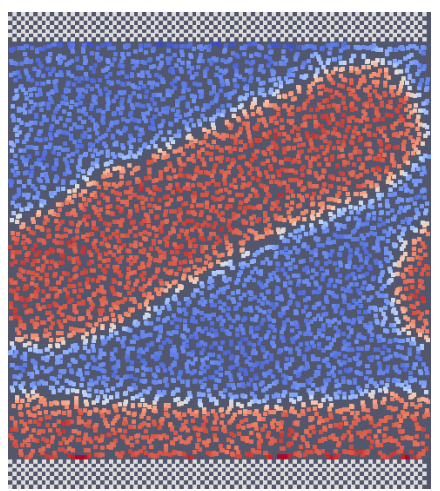

(j) $t=400$

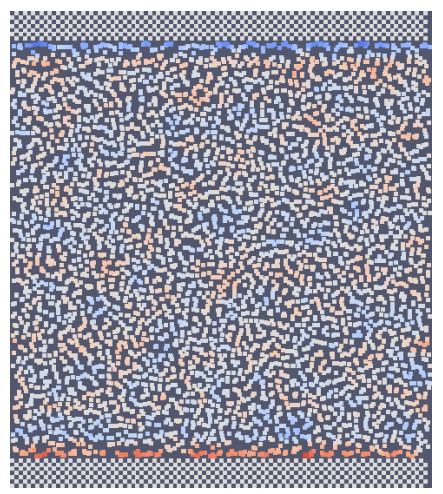

(b) $\quad t=5$

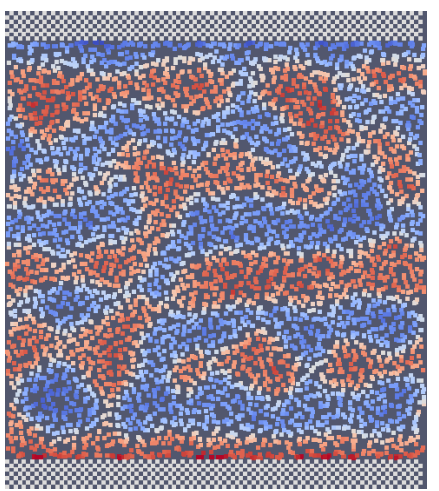

(e) $\quad t=25$

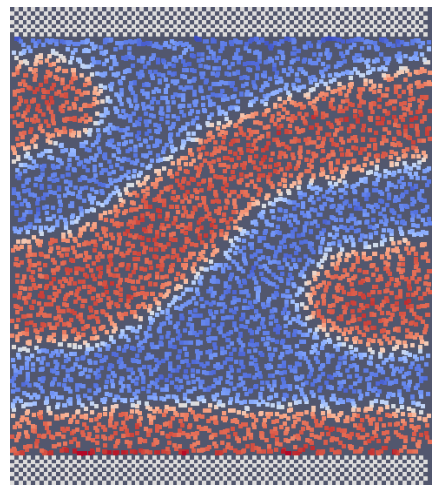

(h) $\quad t=200$

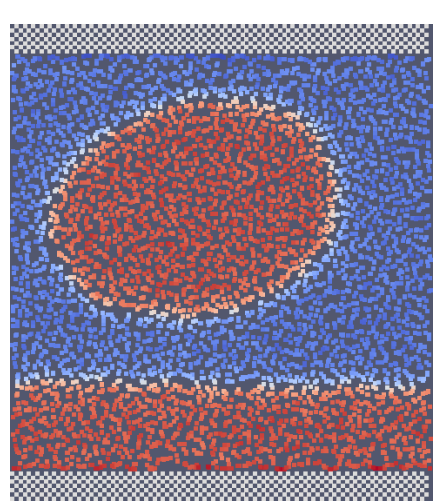

(k) $\quad t=1150$

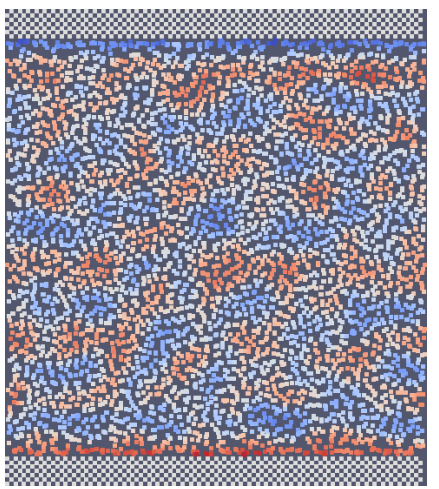

(c) $\quad t=10$

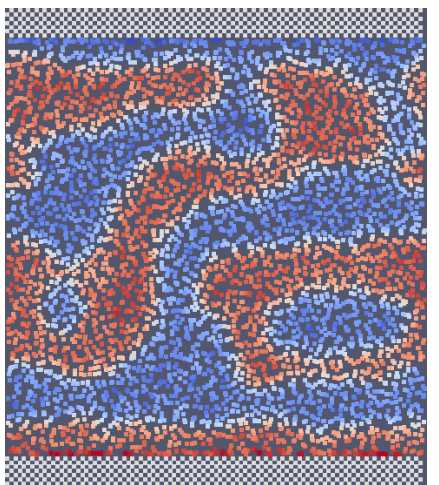

(f) $\quad t=50$

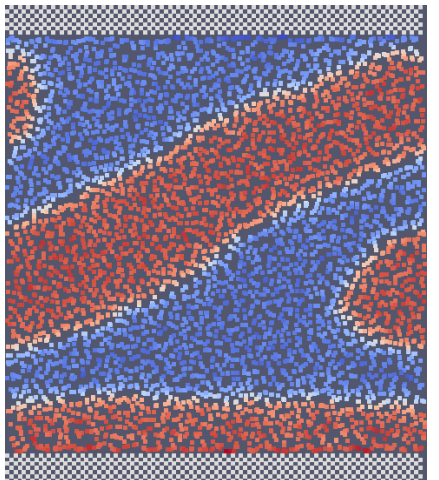

(i) $t=300$

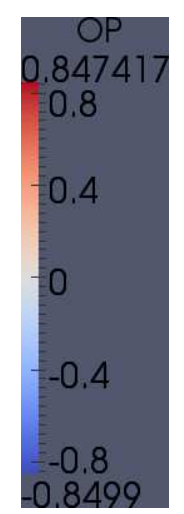

Figure 6.6: Domain growth for the symmetric polymer blend of size $50 \times 50$ with $\sigma=-0.1$ and $v=2.0$. 


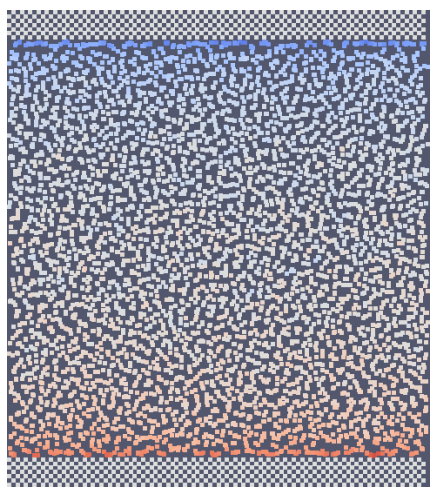

(a) $t=0$

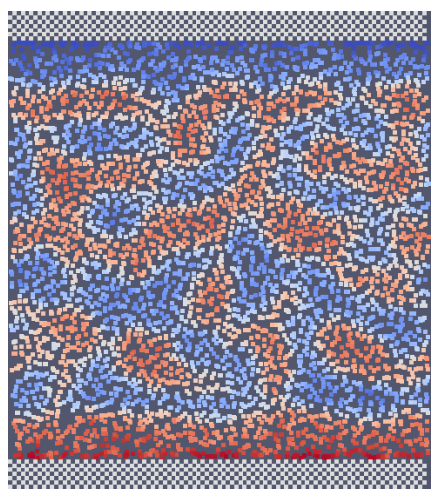

(d) $\quad t=15$

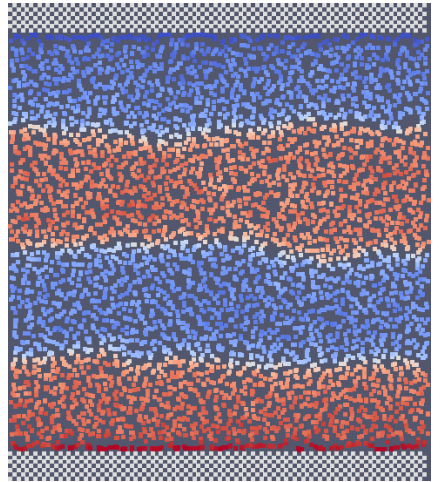

(g) $t=100$

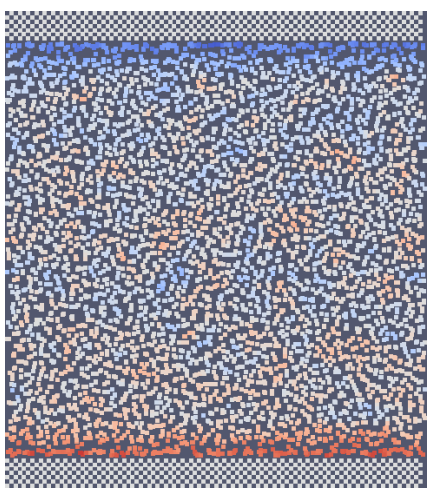

(b) $t=5$

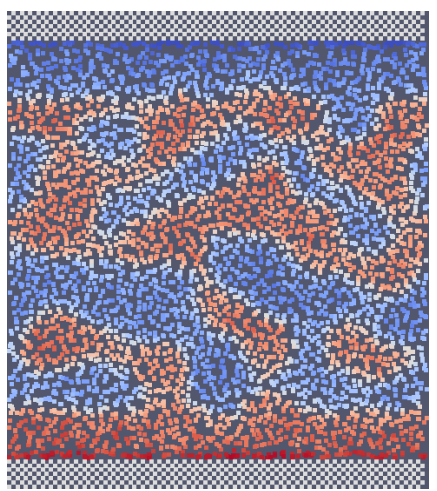

(e) $t=25$

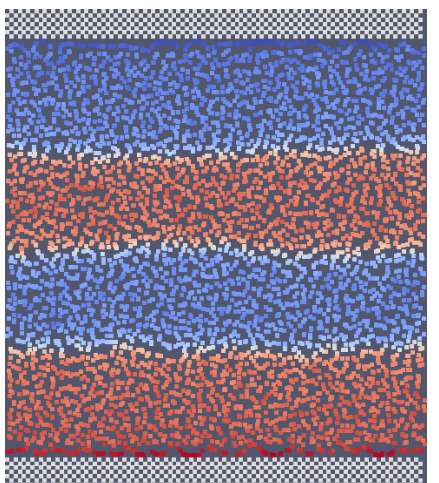

(h) $t=200$

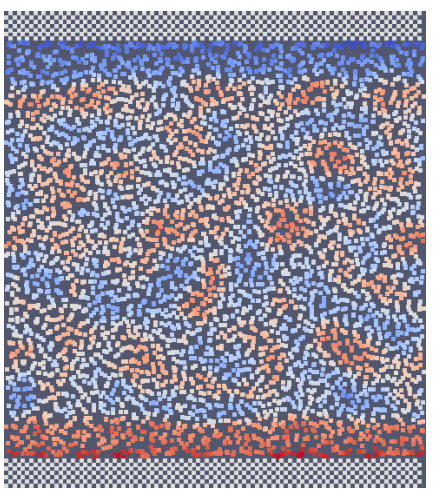

(c) $t=10$

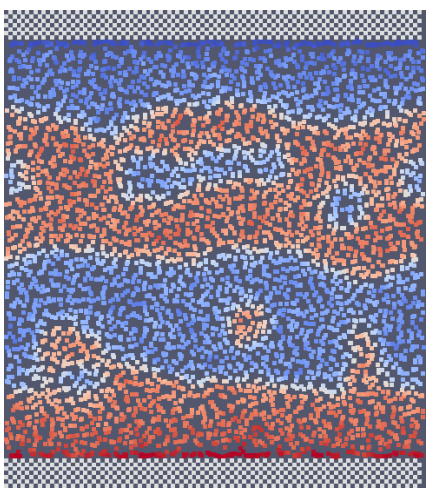

(f) $\quad t=50$

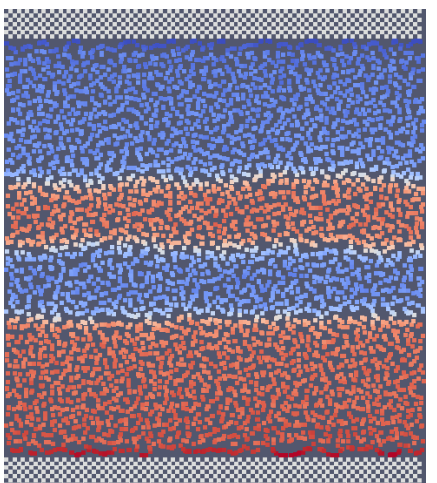

(i) $t=400$

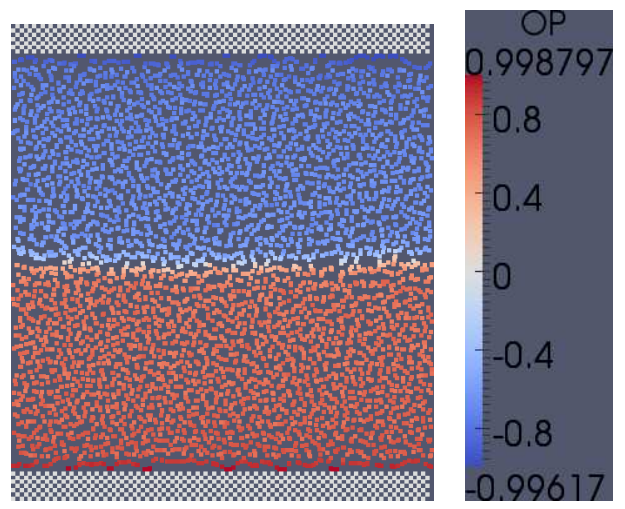

(j) $t=900$

Figure 6.7: Domain growth for the symmetric polymer blend of size $50 \times 50$ with $\sigma=-0.5$ and $v=1.0$. 


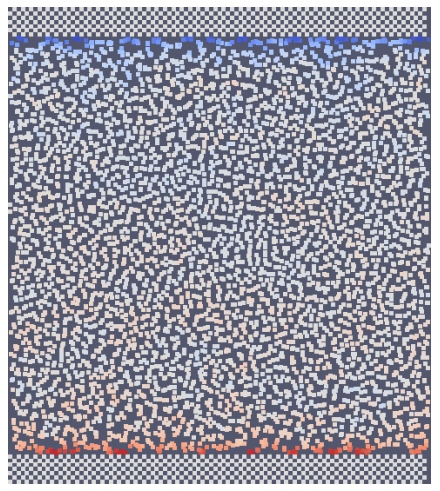

(a) $t=0$

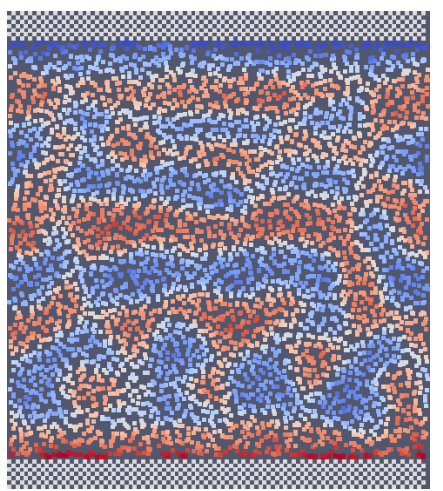

(d) $\quad t=15$

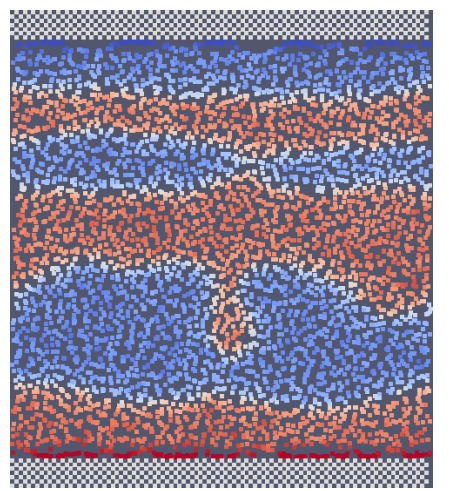

(g) $\quad t=100$

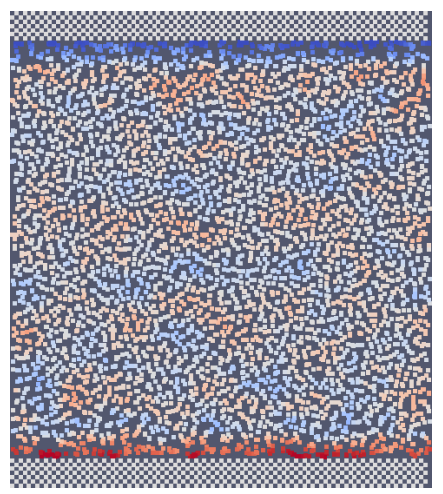

(b) $\quad t=5$

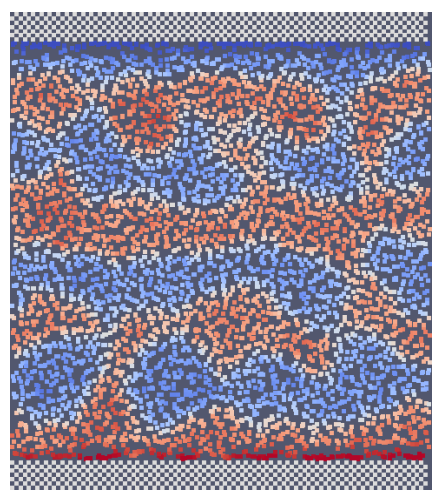

(e) $\quad t=25$

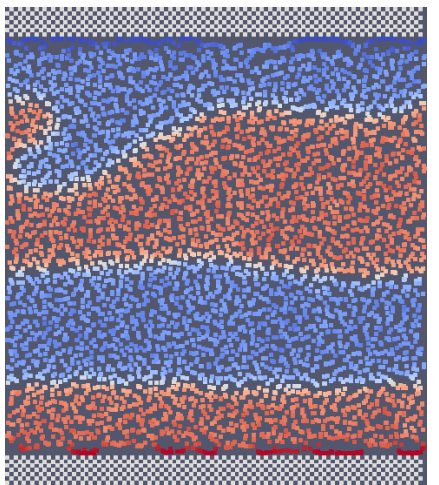

(h) $\quad t=200$

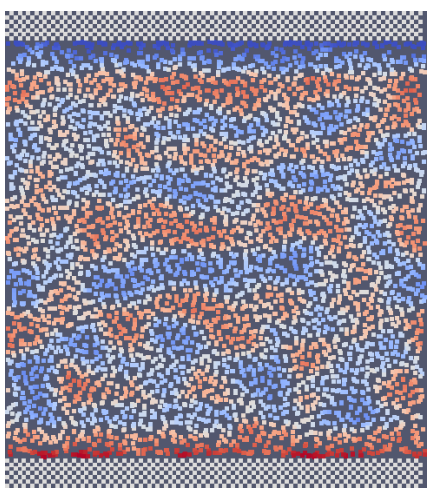

(c) $\quad t=10$

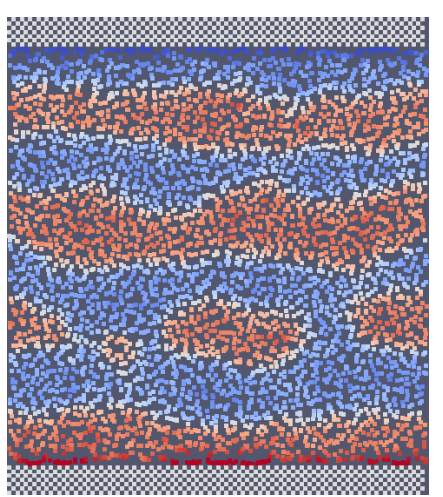

(f) $\quad t=50$

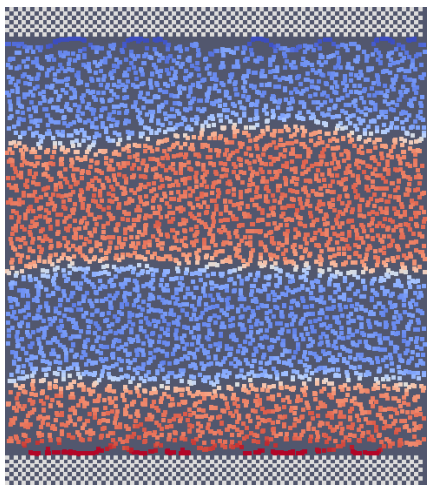

(i) $\quad t=400$

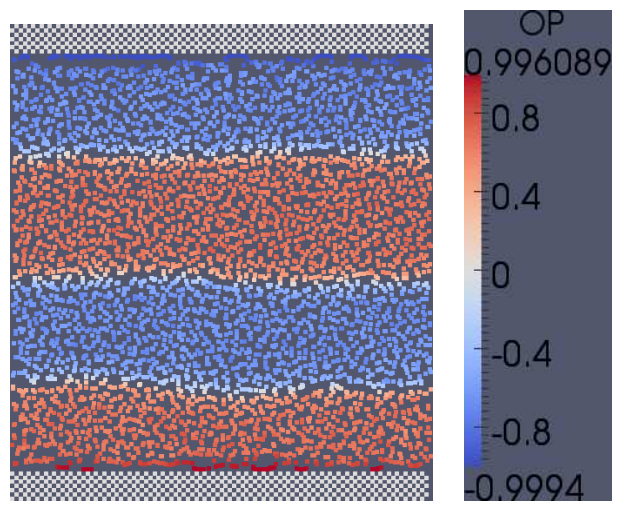

(j) $t=900$

Figure 6.8: Domain growth for the symmetric polymer blend of size $50 \times 50$ with $\sigma=-0.5$ and $v=2.0$. 


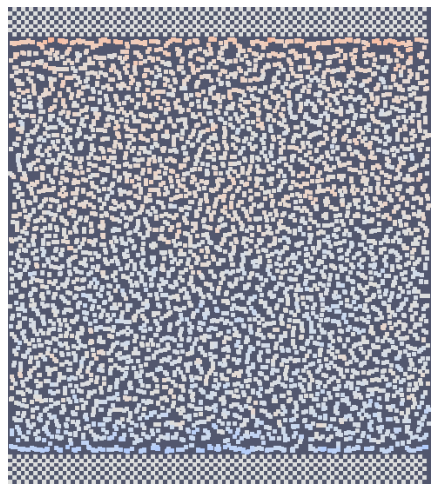

(a) $t=0$

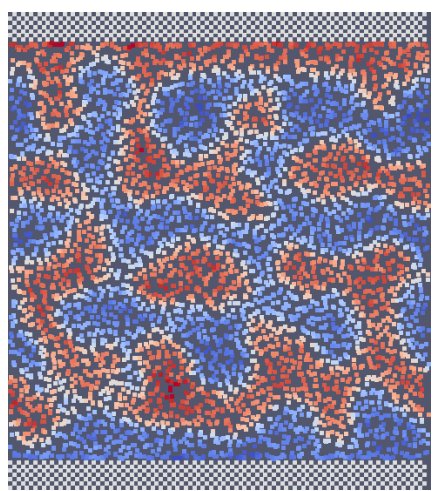

(d) $\quad t=25$

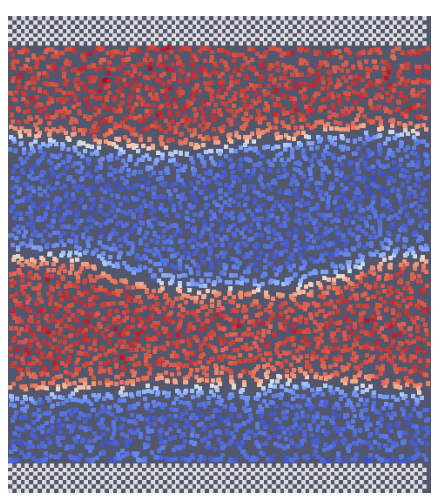

(g) $t=400$

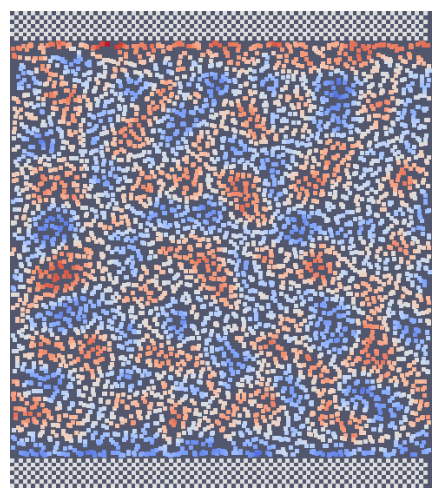

(b) $t=10$

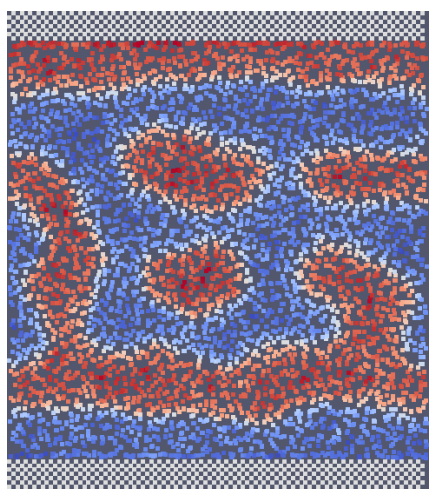

(e) $t=50$

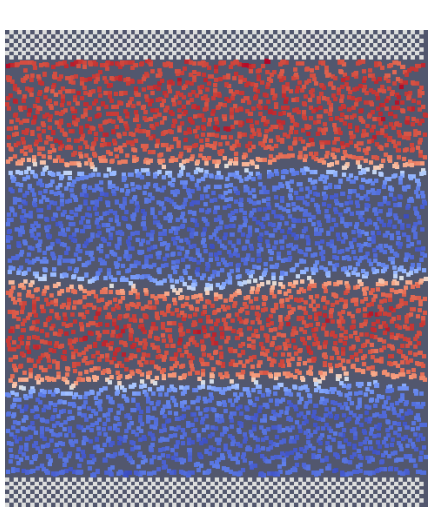

(h) $\quad t=1150$

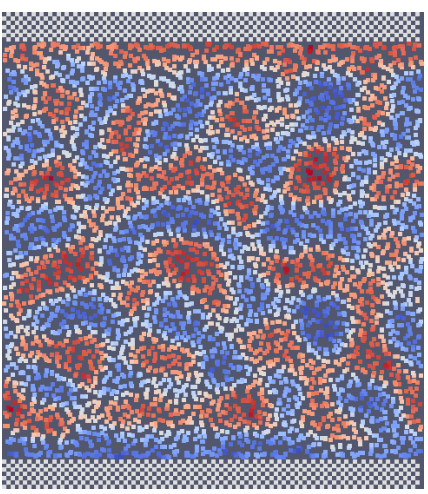

(c) $t=15$

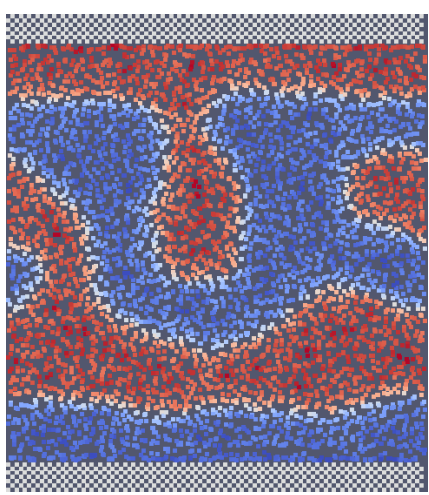

(f) $t=100$

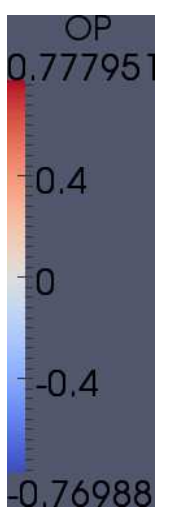

Figure 6.9: Domain growth for the symmetric polymer blend of size $50 \times 50$ with $\sigma=0.1$ and $v=1.0$. The alignment of the phase is opposite to that of $\sigma=-0.1$. 


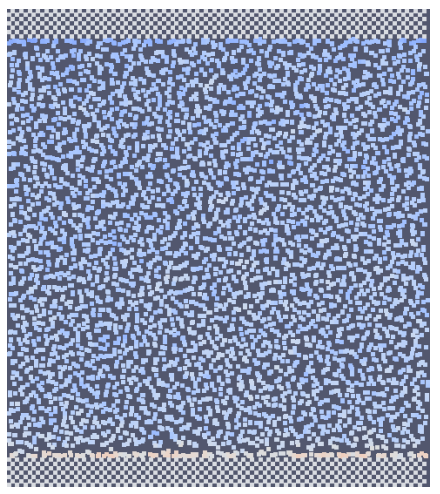

(a) $t=0$

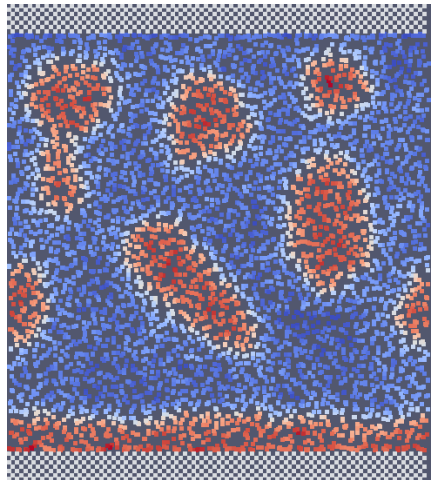

(d) $t=50$

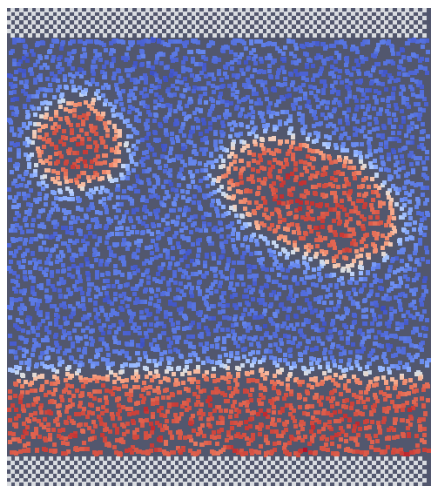

(g) $\quad t=300$

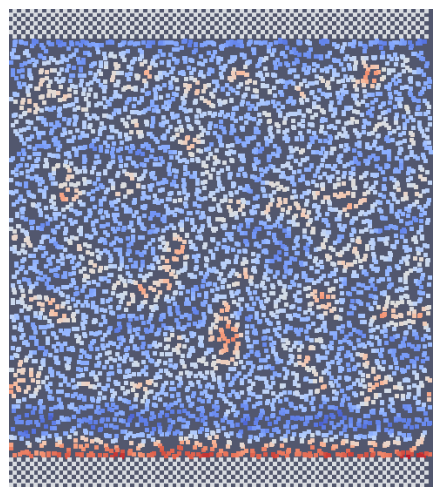

(b) $t=10$

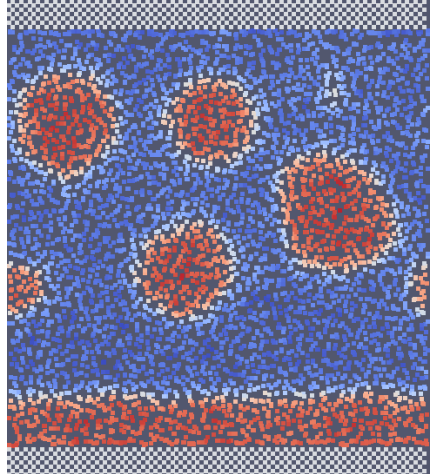

(e) $t=100$

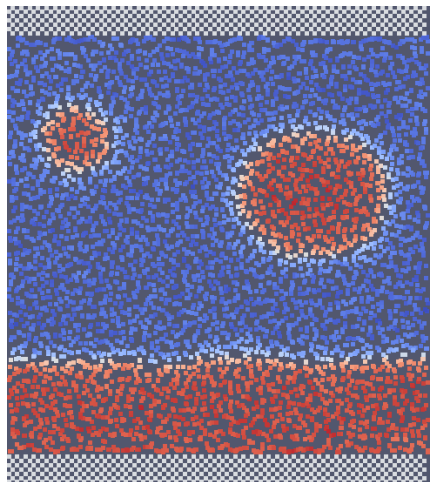

(h) $t=400$

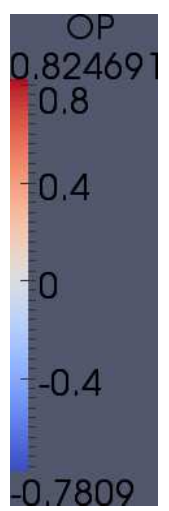

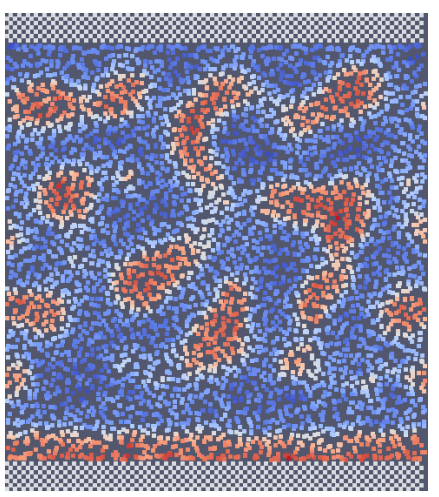

(c) $t=25$

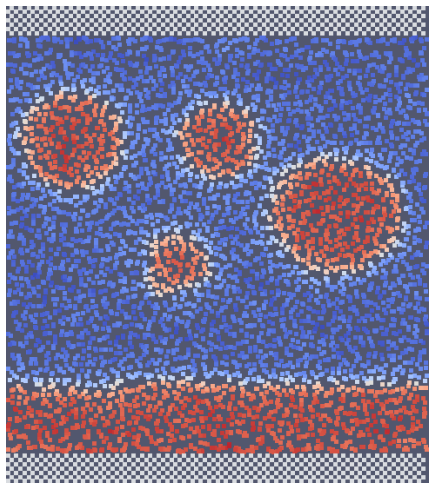

(f) $t=200$

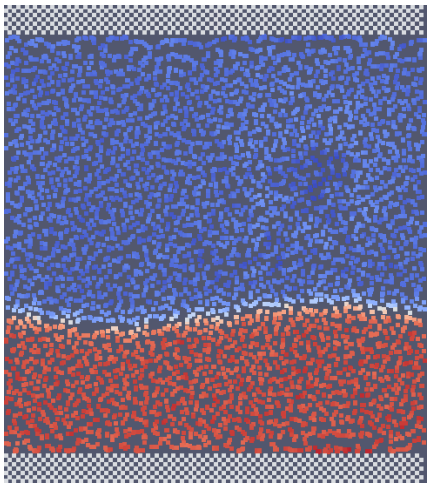

(i) $t=900$

Figure 6.10: Domain growth for the asymmetric polymer blend of size $50 \times 50$ with $f=0.4, \sigma=0.1$ and $v=1.0$. 


\subsubsection{Blend on a Substrate}

Here we test the usefulness of the SPH method for modelling polymer blends with free surfaces. To start with we consider a polymer blend on a substrate (with the wall removed at the upper surface). The same boundary force given by Eq. (6.5.2) was used to model the hardcore repulsion between the fluid particles and the wall to prevent the fluid particles from penetrating the wall. The fluid-wall interaction was implemented as discussed in the previous section, via Eq. (6.58). In all previous cases the system dimensions $\left(L_{x}, L_{y}\right)$ did not change in time and hence constructing the cell grid $\left(M_{x}, M_{y}\right)$ for linked-list purposes had to be done only once at the beginning of the simulation. But in the present case, the surface of the system is free to deform in the $y$ direction, and hence its dimensions in this direction are time dependent. This means that the cell grid has to be constructed at every single timestep to build the linked-list. Hence, these simulations are computationally more intensive compared to fixed domain simulations.

This system differs from the two substrate case in one other aspect. In the two substrate case we need not bother about the momentum transfer to the particles in the normal direction due to the boundary force implementation. Since the system was bounded in both the $y$ directions, any initial momentum transfer would not lead to particles drifting away from the substrates. However, in the present case, the system does not have any upper boundary and it is free to deform in that direction. Therefore, the momentum transferred in the normal direction due to the boundary condition can lead to drifting of the whole system away from the substrate in the positive $y$ direction.

To oppose the system from drifting in positive the $y$ direction, we have included a gravity-like term in the equation of motion:

$$
\ddot{y}_{\alpha}=\left[\sum_{\beta}\left(\frac{\underline{\underline{\varrho}}_{\alpha \beta}}{\rho_{\alpha}^{2}}+\frac{\underline{\underline{\varrho}}_{\beta \alpha}}{\rho_{\beta}^{2}}\right) \cdot \nabla_{\alpha} W_{\alpha \beta}\right]_{y}-g,
$$

where the last term $-g$ acts in the downwards direction and $[\cdots]_{y}$ indicates the $y$ component of the term within the brackets. A range of $g$ values were investigated to find the right choice for $g$ to model this system. Periodic boundary conditions are applied in the $x$ direction. It is important to note here that we have not implemented any surface tension term at the top, free surface. Although this is possible to do, it requires an additional calculation which we have not implemented as yet. We will discuss this in the Future Work section, below. Thus the vacuum-polymer blend surface tension is the same for both phases. 
In Fig. 6.11, we show the results for the case $\sigma=-0.1, v=1.0$ and $g=0.0001$. In this case, the $A$ particles were found to move towards the substrate and as a result the $B$ particles moved away from the substrate. The upper surface of the system freely deformed as can be seen from the snapshots of the system. During the course of the simulation, because of the momentum gained due to the boundary force, the particles and eventually the whole system was found to drift in the $y$ direction. This can observed from the system snapshots at time $t=200$. Eventually, due to the gravity term, the blend settles down on the substrate with the preferred phase next to it. Increasing the value of $g$ to 0.0003 has shown to improve the stability by reducing the drift of the system and this can be seen in Fig. 6.12. However, there was still some drift in the system in this case too. Further increasing the $g$ value prevents the system from drifting altogether but it leads to oscillatory fluctuations in the system, in the $y$ direction. This is because large values of $g$ push the particles towards the substrate which will cause the pressure to increase. This eventually results in particles being pushed away from the substrate. However, these oscillations were found to decay with time. 


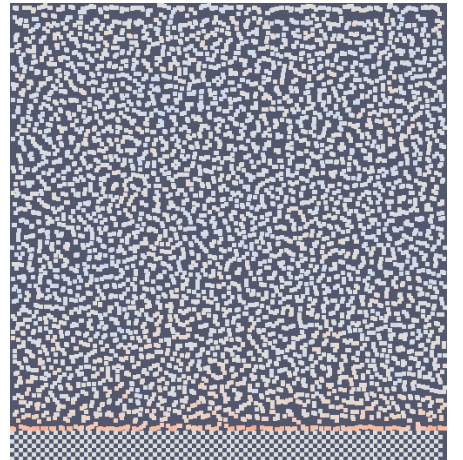

(a) $t=0$

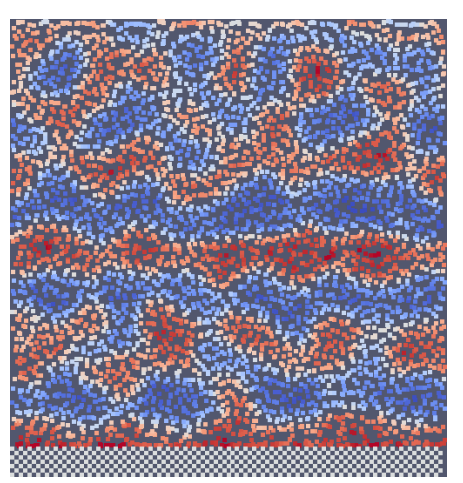

(d) $t=15$

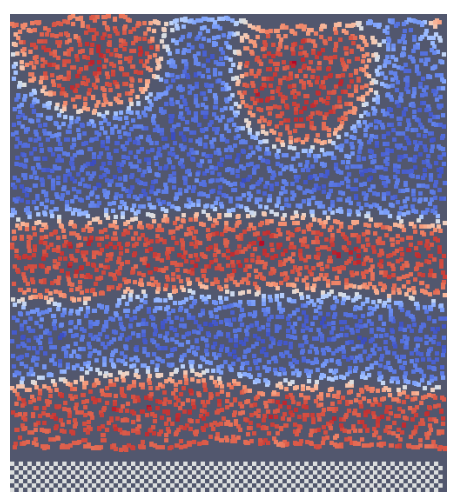

(g) $\quad t=200$

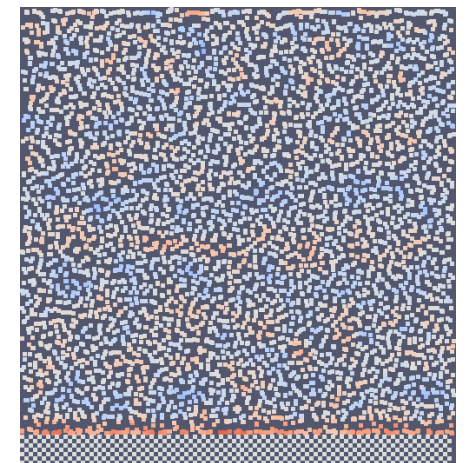

(b) $\quad t=5$

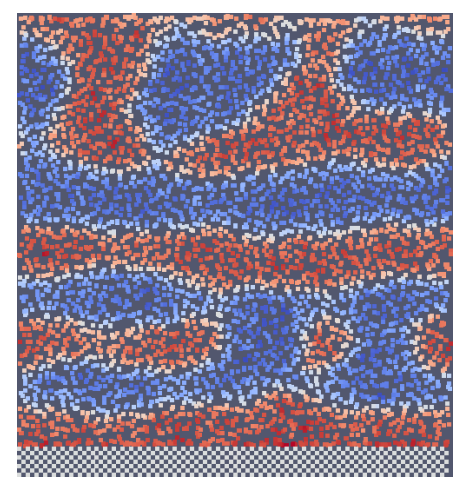

(e) $t=50$

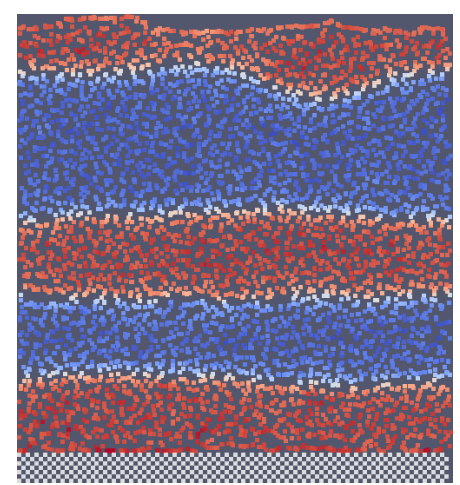

(h) $t=400$

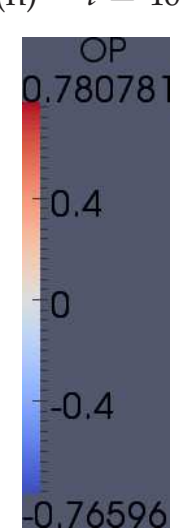

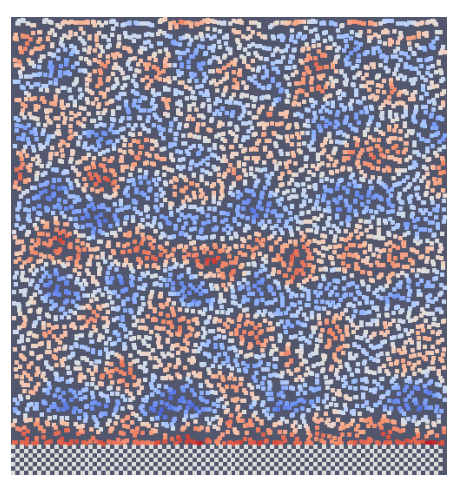

(c) $t=10$

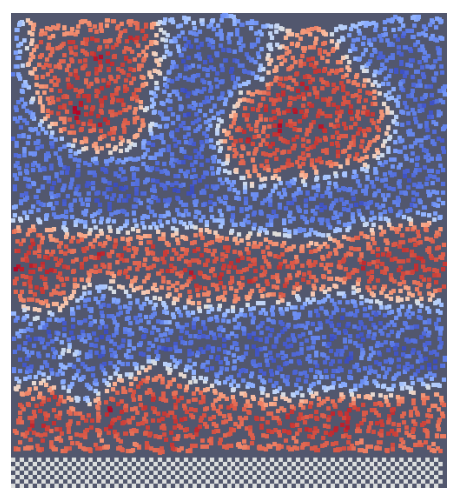

(f) $t=100$

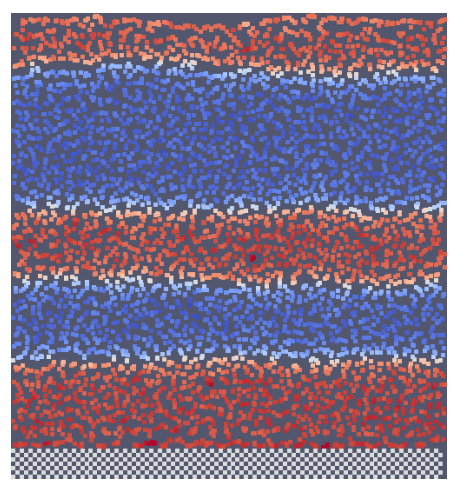

(i) $t=900$

Figure 6.11: Phase separation for a $50 \times 50$ polymer blend on a substrate with $\sigma=-0.1, v=1.0$ and $g=0.0001$. 


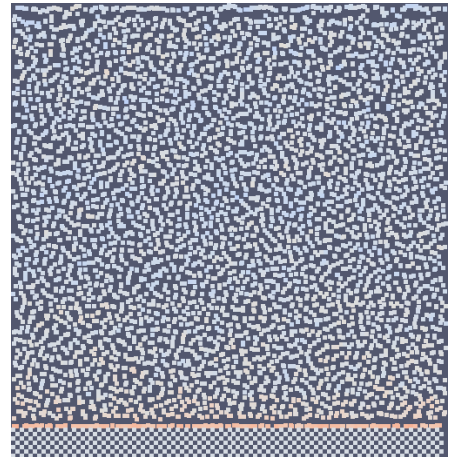

(a) $\quad t=0$

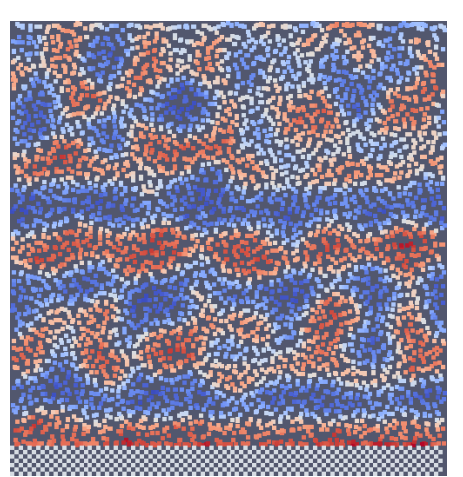

(d) $\quad t=15$

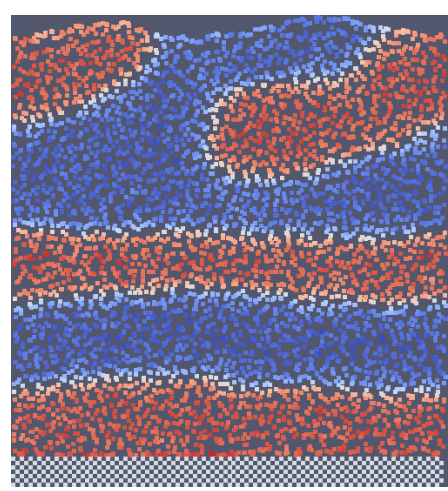

(g) $\quad t=200$

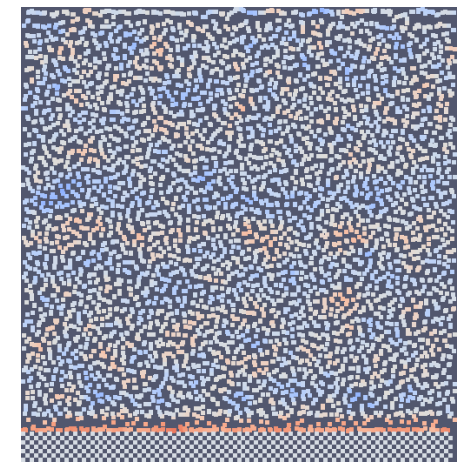

(b) $\quad t=5$

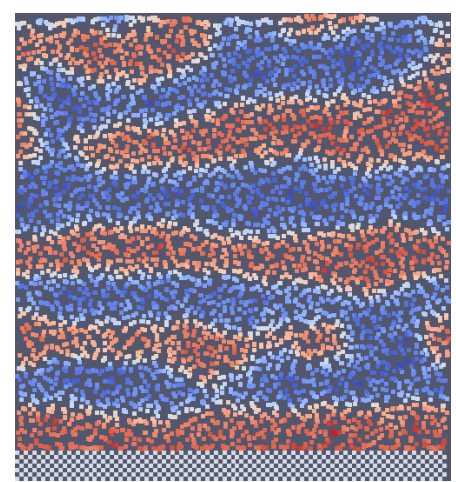

(e) $\quad t=50$

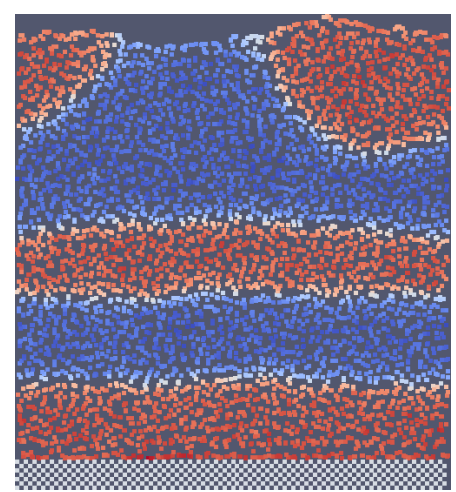

(h) $\quad t=400$

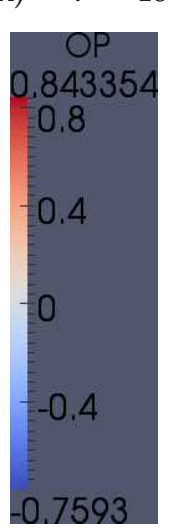

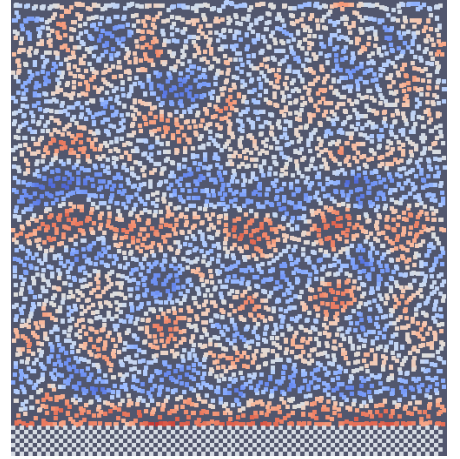

(c) $t=10$

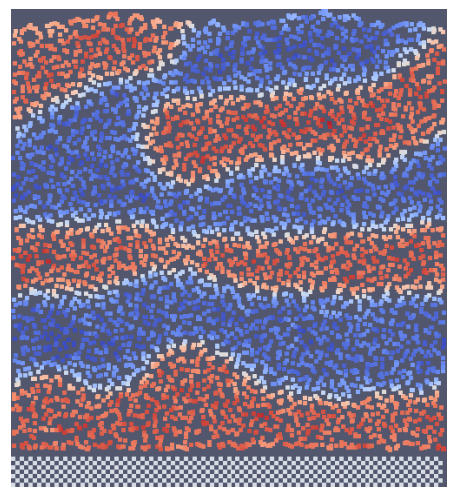

(f) $\quad t=100$

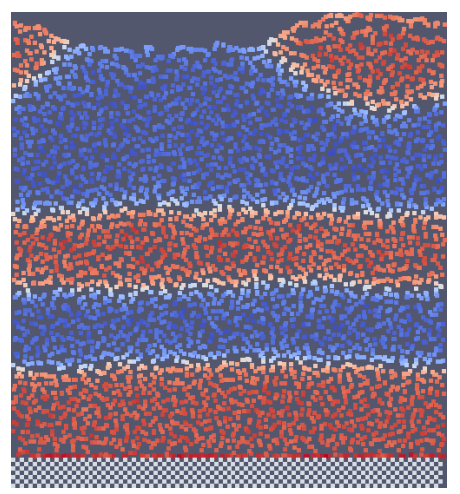

(i) $\quad t=900$

Figure 6.12: Phase separation for a $50 \times 50$ polymer blend on a substrate with $\sigma=-0.1, v=1.0$ and $g=0.0003$. 


\subsubsection{Blend with Free Surface}

Here we study polymer blends with freely deformable surfaces. Since the surface of the system is free to deform in both directions, the system dimensions change in time. This leads to constructing the cell grid every single timestep as mentioned in the previous section. Once again, we emphasize there is no difference in surface tension between the two components of the polymer blend and vacuum.

The phase separation process for one of the model systems is shown in Fig. 6.13. The spatial domain was found to freely deform. In the long time limit the $A$ and $B$ phases separated on macroscale forming a dumbbell shape. The shape of the dumbbell is symmetric (with respect to the interface between the phases) which we would expect since each component has the same surface tension with vacuum. Hence, the system has formed a stable (minimum) free energy state. Of course the structure of the macroscopic phase separated system will depend on the parameters in the chemical potential, Eq. (6.41). Simulations for larger systems were also carried out and the results are shown in Fig. 6.14. Formation of $A$ and $B$ rich domains was observed. However, to reach macroscopically separated phases the simulations have to be run for very long times and it is quite probable the final state at $t=900$ is only metastable. Simulations were also carried out for the asymmetric case of $f=0.4$. Droplet like minority domains were observed growing in time and this result is consistent with the earlier results for the same case. The results are shown in Fig. 6.15.

The simulations in this section has demonstrated the capability of the present technique to model freely deforming surfaces. Such results would be extremely difficult, if not impossible, with any grid-based technique. 


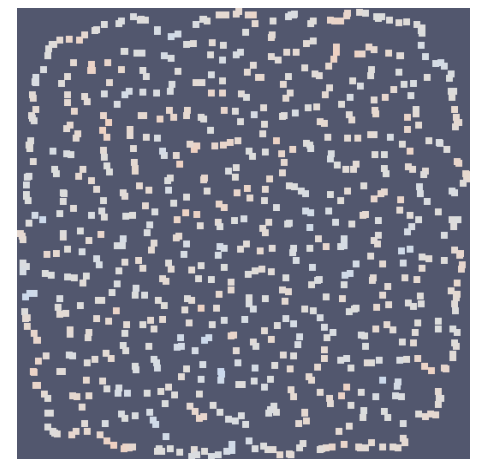

(a) $t=0$

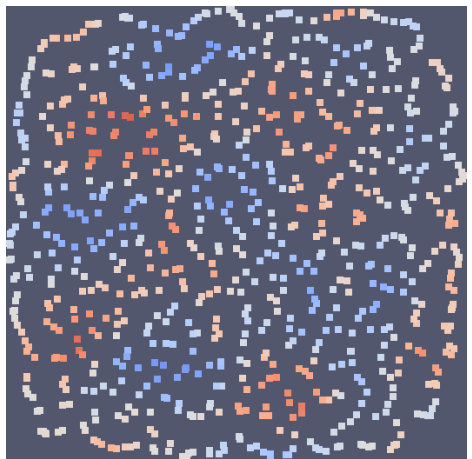

(b) $\quad t=10$

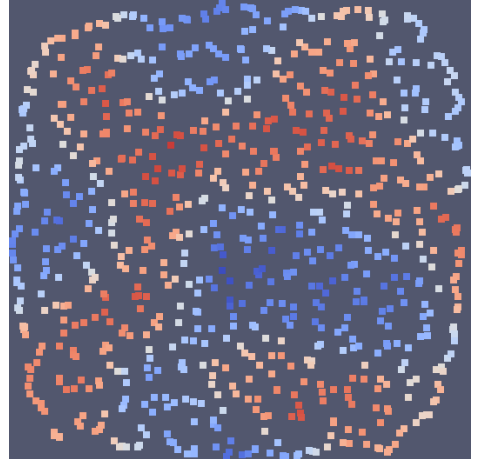

(c) $\quad t=25$

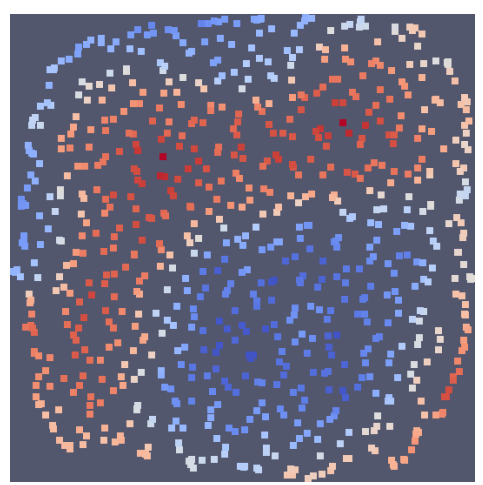

(d) $\quad t=50$

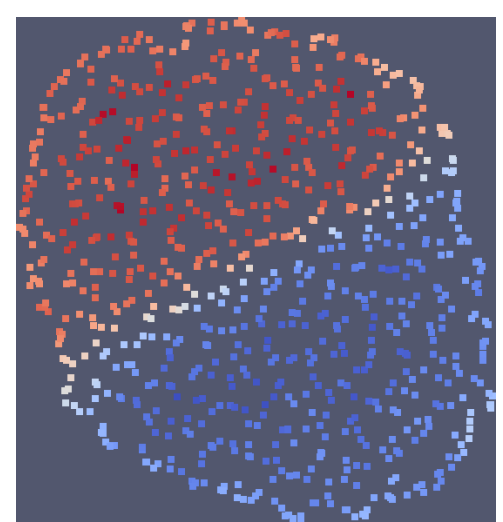

(f) $\quad t=200$

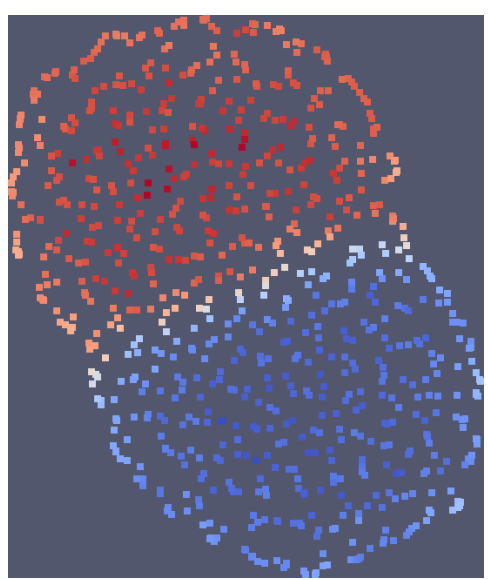

(h) $\quad t=900$

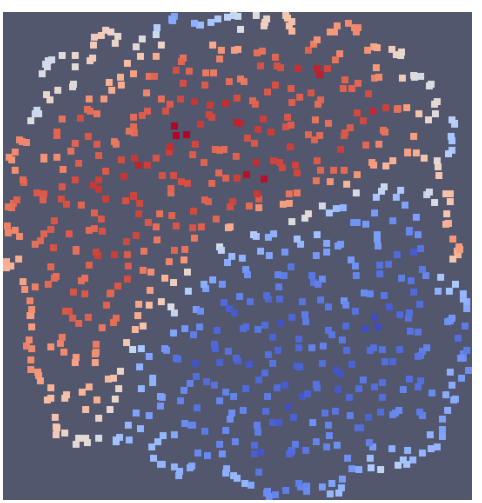

(e) $t=100$

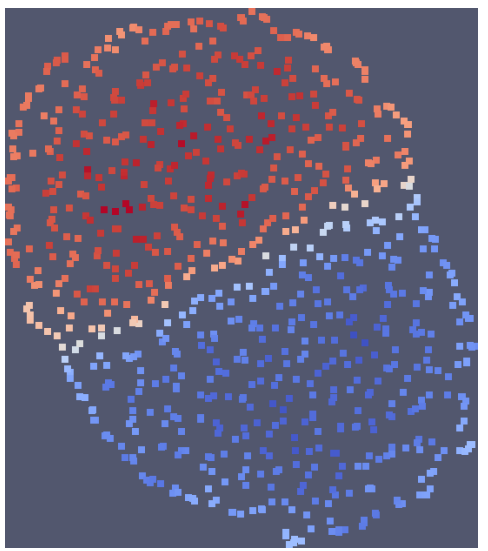

(g) $\quad t=400$

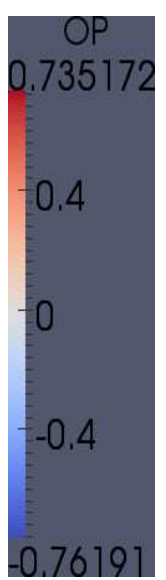

0.76191

Figure 6.13: The phase separation process for a $20 \times 20$ polymer blend with freely deformable surface. 


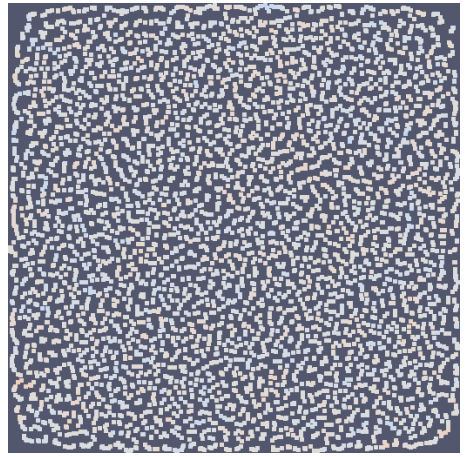

(a) $t=0$

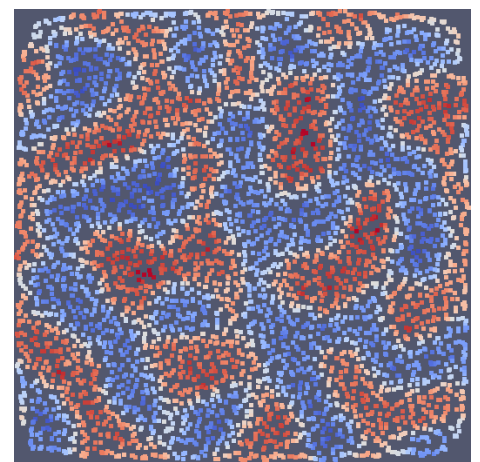

(d) $t=25$

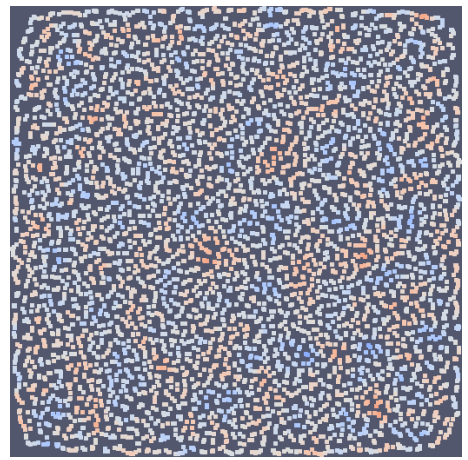

(b) $t=5$

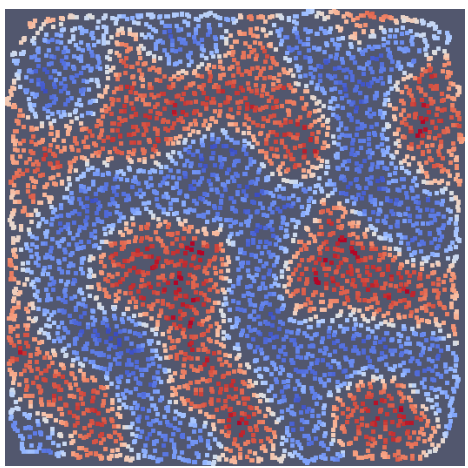

(e) $t=50$

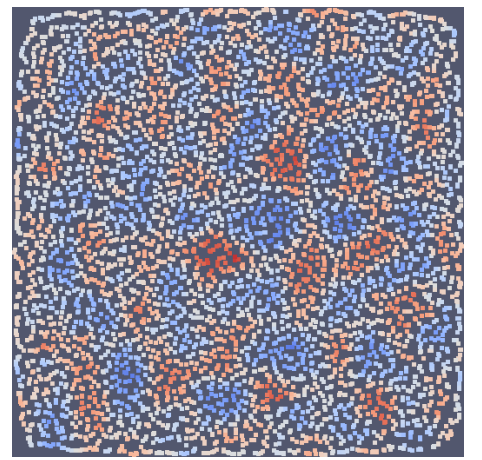

(c) $t=10$

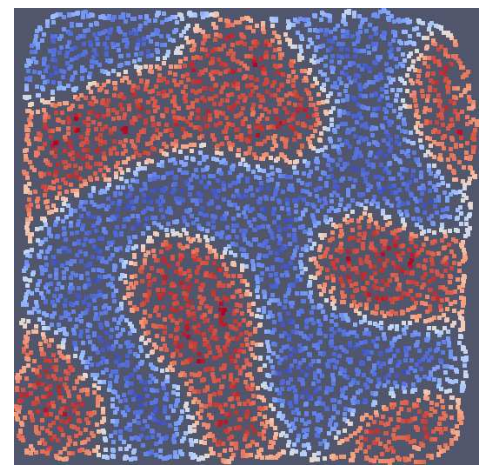

(f) $t=100$

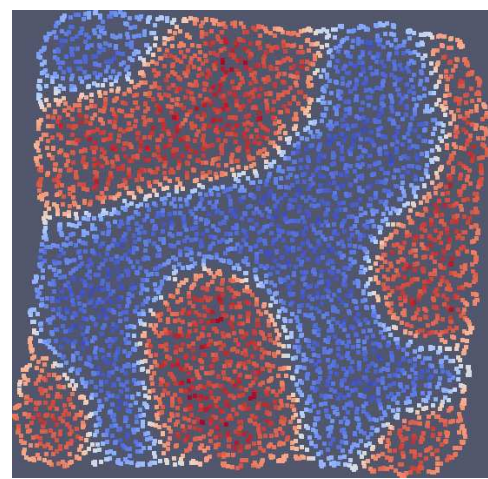

(g) $t=200$

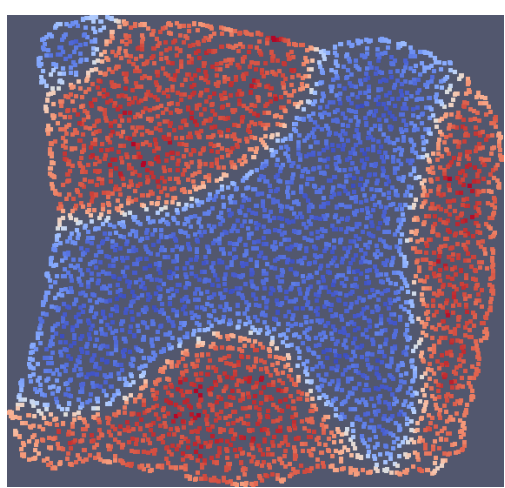

(i) $t=400$

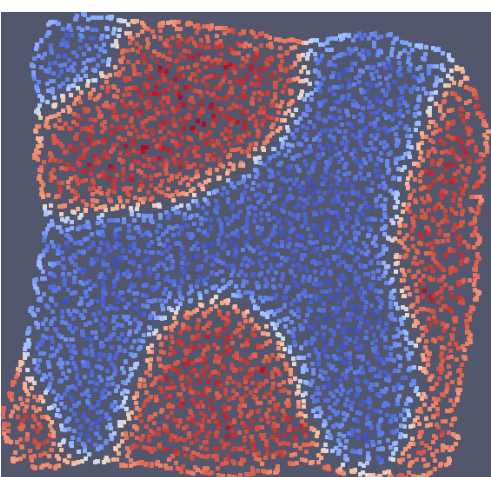

(h) $t=300$
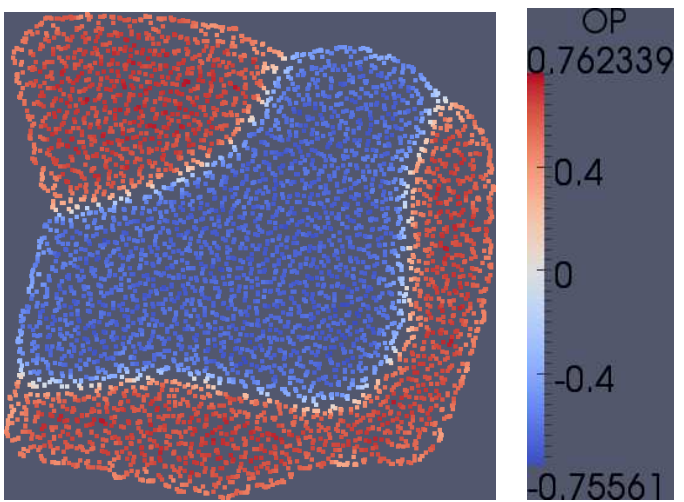

(j) $t=900$

Figure 6.14: The phase separation process for a polymer blend of $50 \times 50$ system with freely deformable surface. 


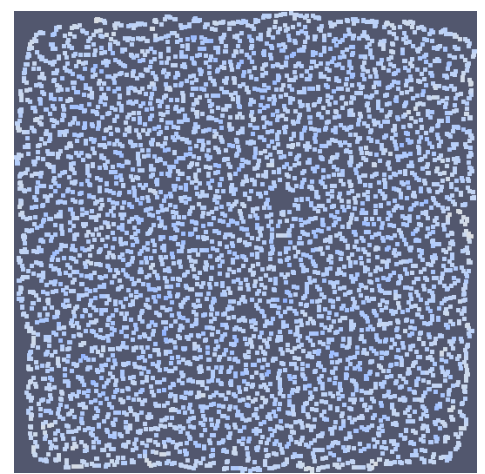

(a) $t=0$

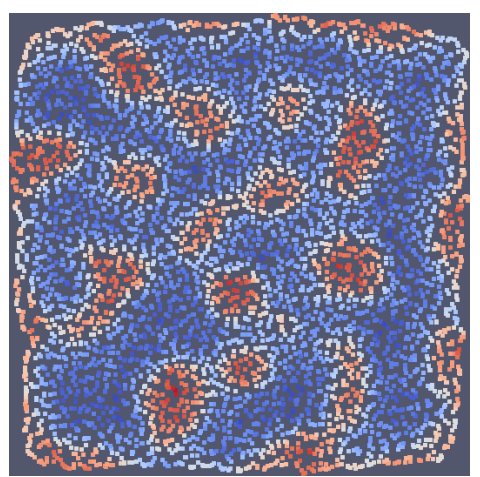

(d) $\quad t=25$

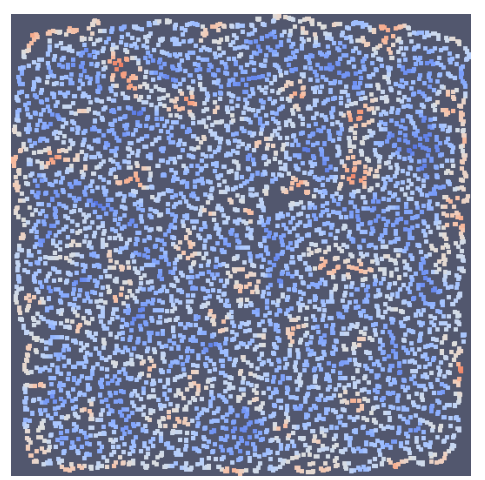

(b) $t=10$

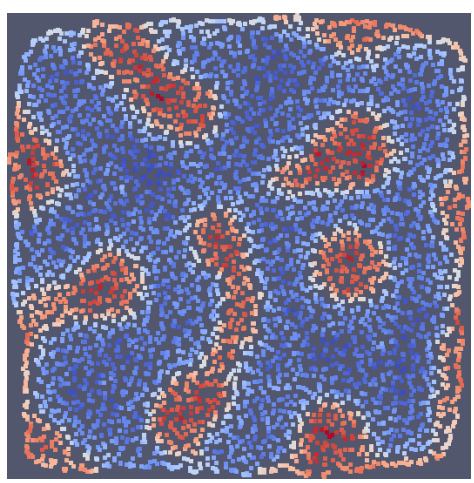

(e) $\quad t=50$

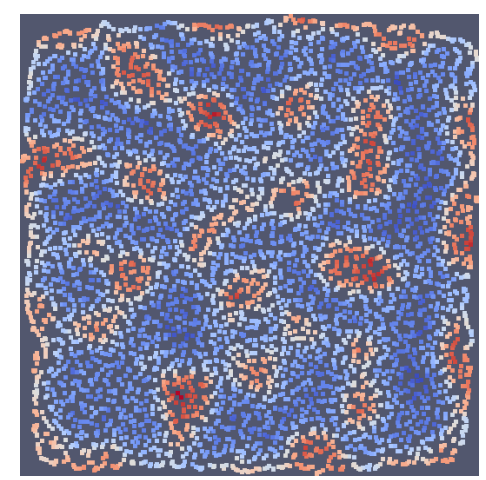

(c) $\quad t=20$

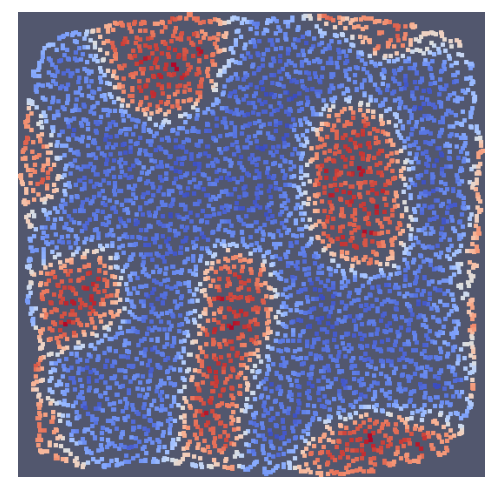

(f) $\quad t=100$

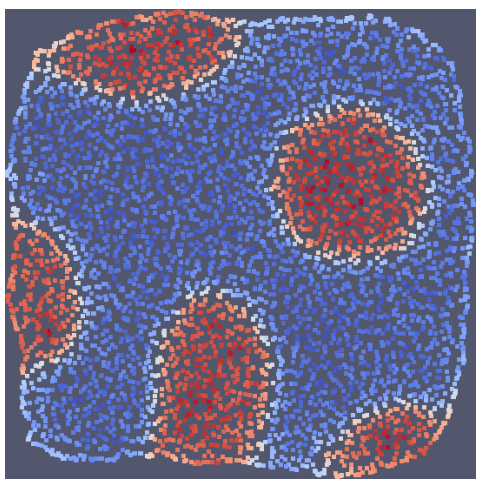

(g) $\quad t=200$

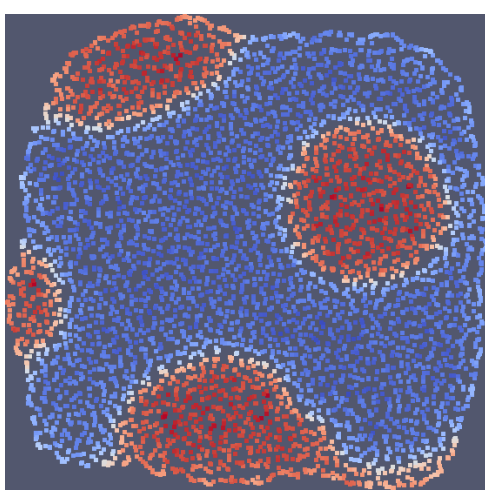

(i) $\quad t=400$

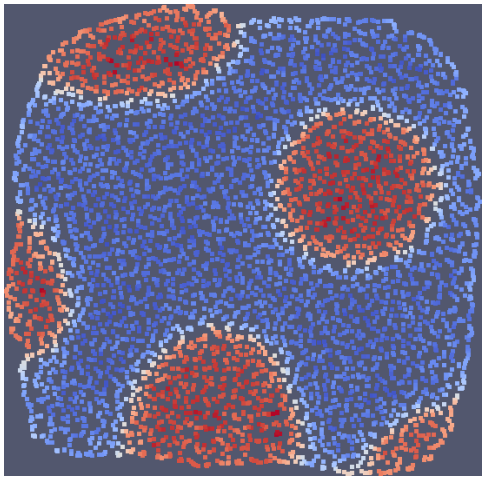

(h) $\quad t=300$

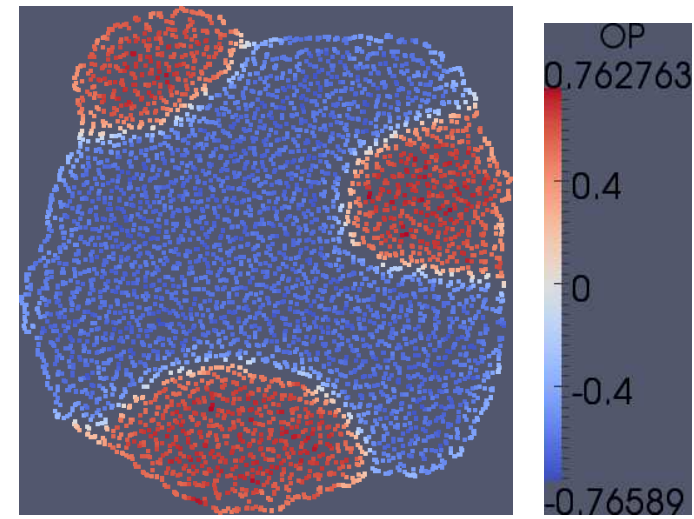

(j) $\quad t=900$

Figure 6.15: The phase separation process for a polymer blend of $50 \times 50$ system with freely deformable surface for the case of $f=0.4$. 


\subsection{Conclusions}

In this chapter we have successfully applied the SPH method to study the phase separation process in polymer blends under a variety of boundary conditions. To start with phase separation in both symmetric and asymmetric blends was studied with periodic boundary conditions applied in both directions. The phase separation process was observed to take place simultaneously system-wide and this confirms that the underlying mechanism is spinodal decomposition. As expected, in the asymmetric blend case of $f=0.4$, growth of a droplet-like minority phase was noticed.

Then, we studied the phase separation in polymer blends confined between hard walls. Simulations were conducted for different surface energy-strengths and forms (short-range and long-range). In general, for symmetric polymer blends lamellae-type phases were found to grow parallel to the walls, indicating signs of macrophase separation at long times. One exception from the above trend (lamellae-type phase growth) was noticed in $v=2.0$ case and we attribute this to the system being stuck in one of the metastable states. In the asymmetric blend case $(f=0.4$ ), growth of droplet-like minority domains was observed away from the walls. Near the walls the surface energy influence on the phase separation process was evident leading to phase alignment parallel to the walls. In the short-range surface energy cases $(v=2.0)$, due to rapid decline in the surface energy as a function of distance from the walls, macrophase separation took longer than the long-range surface energy cases $(v=1.0)$. Increasing the surface energy strength has shown to speed up the phase separation process. By controlling the sign of surface energy term with respect to the phase, preferred phase alignment was achieved.

The utility of the SPH method in modelling phase separation of freely deforming blends on a substrate was tested next. A range of gravity-like forces $(g)$, to prevent the system from drifting away from the substrate, were tested and reasonable behaviour was found for values between $g=0.0001$ to 0.0003 . For these values of $g$ a small drift in the system was observed during the intermediate times. But eventually the system was found to settle down on the substrate and the rate at which this happens (settling process) was found to be proportional to the magnitude of $g$. For values of $g>0.0005$, the system was found to oscillate in the $y$ direction, indicating compression of the fluid. The usual phase separation process was observed in all these simulations and the free deformation of top surface was evident (see Fig. 6.12). 
Finally, the phase separation of blends without any constraints (confinements) and without periodic boundary conditions was studied. The blend surface was found to deform freely in both directions. For the given set of parameters $\varepsilon$ and $K$, and for $20 \times 20$ system at long times a dumbbell-type macrophase morphology was observed. Similar equilibrium macrophase separation is expected for $50 \times 50$ system at long times.

\subsubsection{Future Work}

The major improvement of the present model as applied to polymer blends is the capability to model different surface tensions in the case where we have free (boundary) surfaces. That is, we require the A monomer-vacuum surface tension to be different from the B monomer-vacuum surface tension. To do this we need to be able to identify the (free surface) boundary particles and then apply a suitable surface tension to these particles. While this implementation is slightly more difficult than what we presently have done, we have a good understanding as to how to do this. Essentially, boundary particles have fewer nearest neighbours than bulk particles and we may use this property to identify them. Moreover, with the kernel implementation in SPH, we naturally keep a tab on the number of nearest neighbours each particle possesses.

We implemented the GL-type free energy in these simulations. This method works very well close to the critical temperature thus local order parameters $\phi \approx 0$ and for small molecular weight blends. The next step will be to use the FHdG free energy functional given by Eq. (6.15) with the Flory-Huggins free energy of mixing (Eq. (6.13)). Here the asymmetry in blend composition directly enters via the Flory-Huggins free energy. This model, in principle, should be applicable over wider range of temperatures (away from critical temperature).

We believe our method can be extended to study self-assembly in diblock copolymer systems. Block copolymer melts exhibit exotic self-assembled patterns, with a periodic pattern wavelength. If the system dimensions are not an integer multiple of the periodic pattern wavelength, it gives rise to frustration effects, as the system cannot attain its equilibrium morphology. This in turn can give rise to new or variants of existing morphological patterns [168]. Commonly used grid-based methods to study these systems rely on trial and error to

find suitable dimensions for the system. For example, as pointed out in the Introduction chapter, a diblock copolymer melt spin coated onto a flat substrate yields a deforming free surface and ultimately an island and hole structure [157]. Our model can potentially address these issues. The free energy for diblock 
copolymer systems on a substrate has the following form [157]:

$$
\frac{F}{k_{B} T}=\int_{V} d \mathbf{r}\left[-\varepsilon \phi^{2}+\frac{u}{2} \phi^{4}+\frac{K}{2}|\nabla \phi|^{2}+B \int_{V^{\prime}} d \mathbf{r}^{\prime} G\left(\mathbf{r}, \mathbf{r}^{\prime}\right) \phi(\mathbf{r}) \phi\left(\mathbf{r}^{\prime}\right)\right]-\frac{F_{\text {surface }}}{k_{B} T},
$$

and as we can see this equation is just an extension of Eq. (6.56). The additional term ( $\left.\int_{V^{\prime}} d \mathbf{r}^{\prime} \cdots\right)$ in the above equation, involving $G$ which is a Green's function, models the connectivity between two types of polymers in the diblock copolymer. The complexity with this term is that the Green's function is of a long range nature and hence becomes computationally expensive to evaluate. 


\section{Chapter 7}

\section{Conclusions}

Polymer systems are very complex materials that are vital in a wide range of applications both in industry and biology. In this thesis we studied certain polymer systems that are of interest in nanotechnology. We employed different simulation techniques to study these systems and the simulation findings were complemented with appropriate theoretical analysis, wherever possible. Even though a detailed summary was provided at the end of each of these chapters, here we shall try to condense our main findings from each of these studies and discuss the directions for future work.

\section{Flow over Patterned Surfaces}

This study was intended towards investigating a mechanism to enhance mixing in polymeric fluids in narrow channels. Poiseuille flow over patterned-slip surfaces was found to induce transverse flows indicating mixing. A comparison between the simulation results and continuum analysis has shown good agreement, in general. However, the transverse flows were found to be dependent on the relationship between the wavelength of the slip-pattern $\lambda$ and the size of the chains $R_{g}$. For $\lambda / 2>R_{g}$ transverse flows were well-developed and for $\lambda / 2<R_{g}$ transverse flows were suppressed. When $R_{g}>\lambda / 2$, on average a polymer chain spans two different patterned regions and hence the chains close to the surface behave as if they are adjacent to a homogeneous entity rather than a heterogeneous surface. In such situations the surface patterning will have minimal effects on the flow. Therefore to achieve good mixing it is desirable to have $\lambda / 2>R_{g}$. This condition could be useful when such flows are nanoscale, as at these dimensions polymers become comparable to device dimensions. The continuum theory [1] does not provide any such information. The transverse 
flow was zero in the centre of the channel when upper- and lower-wall patterns were parallel to each other. An off-setting between two wall patterns has shown to induce non-zero transverse flows even in the centre of the channel, in certain regions; thus, suggesting better conditions for mixing.

Next, a time dependent sinusoidal body force was applied on the fluid in conjunction with pattern-slip boundary conditions. This was shown to enhance the transverse flows further (compared to constant body force cases discussed above), as suggested by the continuum theory [1]. However, our simulations differ from the theory in that transverse velocity does not increase with increasing frequency of the body force. The reason for this discrepancy is due to the fact that continuum theory assumes that there is only one single relaxation constant involved whereas our simulations reveal otherwise. It was also found that there is an upper bound on the frequency above which the polymer molecules will not have sufficient time to react to the changes in the body force. Thus suggesting that transverse flows cannot be increased unlimitedly by increasing the frequency of the body force.

\section{Capillary Absorption of Polymer Droplets}

Capillary absorption of small polymer droplets into narrow capillaries was studied using MD simulations. The motivation behind this study was to examine the effect of molecular size on the capillary phenomena in the limit where the chain size becomes comparable with the tube diameter; such situations are often encountered in nanotechnology.

For this purpose, capillary absorption simulations of polymer droplets of the same size (equal number of monomers) but made up of different chain lengths $(N)$ were conducted. Our results show that small $N$ droplets require smaller critical tube radii $\left(R_{t c}\right)$ for absorption than large $N$ droplets. When the chain size of the polymers becomes comparable with that of the tube diameter it results in the deformation of chain conformations which in turn leads to a loss in conformational entropy. Therefore, there is an entropy loss associated with the absorption of polymers in narrow tubes and this loss increases with $N$ thus discouraging capillary uptake of large $N$ droplets. This result can have important implications on the capillary-driven nanoscale phenomena aimed at building polymer nanostructures.

The capillary dynamics were also studied by evaluating the height of the meniscus as a function of time and the results were compared with the Lucas- 
Washburn theory. Our data deviate significantly from the Lucas-Washburn description. Moreover, in one of our simulations for an $N=200$ droplet with $R_{t}=6.69 \sigma$ several metastable states, where the meniscus height did not change for long periods, were observed. Such behaviour cannot be explained by the existing models on capillary dynamics and has not been previously observed. Our findings here once again can be important in capillary-driven methods for nanostructures.

Our results were found to be in qualitative agreement with the proposed theory. The theory also suggests that the capillary uptake of wetting polymer droplets may also be prohibited in sufficiently narrow capillaries. This is in contrast with the general understanding of capillary phenomena, where a wetting liquid is always thought to ascend the tube.

\section{SPH Simulations of Polymer Blends}

The primary goal of this work was to investigate a numerical method for studying phase separation and self-assembly in freely deforming polymer systems. As pointed our earlier, this is of great interest in many self-assembling systems and their related applications. However, in this thesis, as a starting point, we restricted ourselves to the simplest possible binary phase separating polymer system: a polymer blend. Since the emphasis was on testing the applicability of the SPH method to these systems we did not indulge in numerical comparison between our results and well-established results that exist for polymer blends. However, we intend to carry out this in our future work.

As a first step, phase separation in symmetric and asymmetric polymer blends with periodic boundary conditions was studied and the phase behaviour expected was observed. Then, we studied a polymer blend confined between two hard walls by adding an additional surface energy term to model the wall-fluid interaction. Different surface energy strengths and forms were tested out and their impact on the phase separation process was noted. The domain growth away from the walls occurred via spinodal decomposition while close to the walls phase growth was influenced by the wall-fluid surface energy. At long times, macrophase separation was noticed with each phase preferring to align parallel with either of the walls. We showed that by controlling the sign of the surface energy desired phase alignment could be achieved.

Next the SPH method was applied to describe the phase behaviour of polymer blends on substrates, with the top surface free to deform. The phase 
separation was observed as a function of time and during this period the top surface of the system deformed freely. We also studied the phase separation in polymer blends without any boundary conditions. Here for the given choice of parameters, dumbbell-shape phase separation occurred on a macroscopic level.

The SPH method offers the greatest avenue for future investigation. We intend to include a surface tension term (for the case of a freely deforming surface) and also apply our technique to block copolymer melts, for which a number of interesting free surface deformation phenomena have been predicted to occur. We believe the SPH method will be a novel computational tool to investigate free surface polymer problems. 


\section{Appendix A}

\section{Onion-Ring Analysis}

In our MD simulations on polymer droplets, the chains in the bulk of a droplet are expected to behave differently from those ones that are close to the surface. Since our simulations deal with very small droplets, where the surface to volume ratios are high, significant number of monomers/chains reside on the surface. Hence it is important to study the chain properties as function of their radial distance $r$ from the centre of the droplet. This was achieved using what we call the onion-ring analysis. In this method the droplet was divided into radial bins of thickness $d r=0.01 \sigma$, in the radially outward direction. A schematic diagram in which this method is depicted is shown in Fig. A.1.

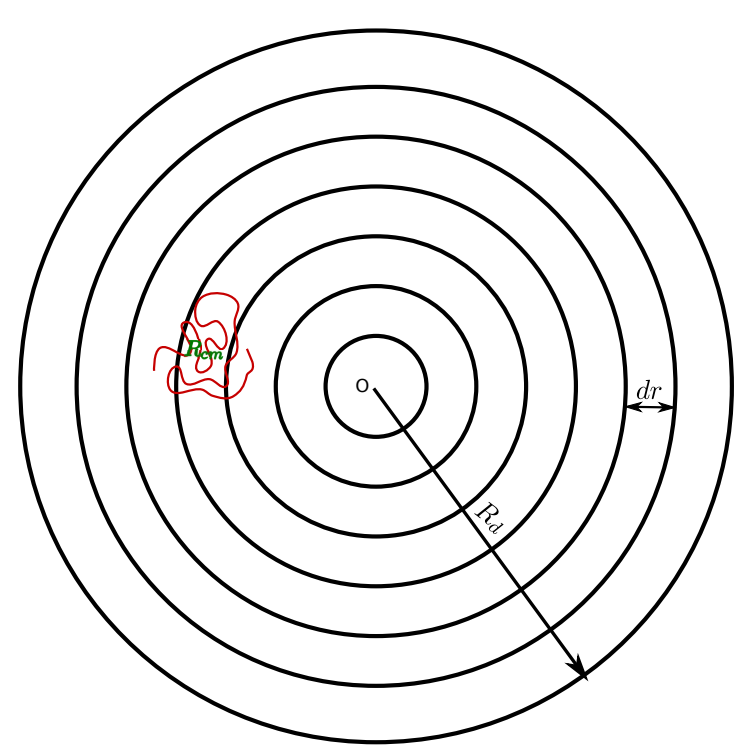

Figure A.1: The droplet is divided into concentric spherical shells of thickness $d r$. An example chain whose centre of mass lies in one the bins is shown here.

For a particular droplet configuration, all the chains in droplet were mapped 
into one of these bins based on their centre of mass positions $(\mathrm{cm})$. After mapping all chains into their respective bins, an average $\left\langle R_{g}\right\rangle$ was computed for each bin, over all the chains whose $\mathrm{cm}$ falls in that bin. This gives us the radial $\left\langle R_{g}\right\rangle$ profile. For better statistics, we calculate the time average of this profile.

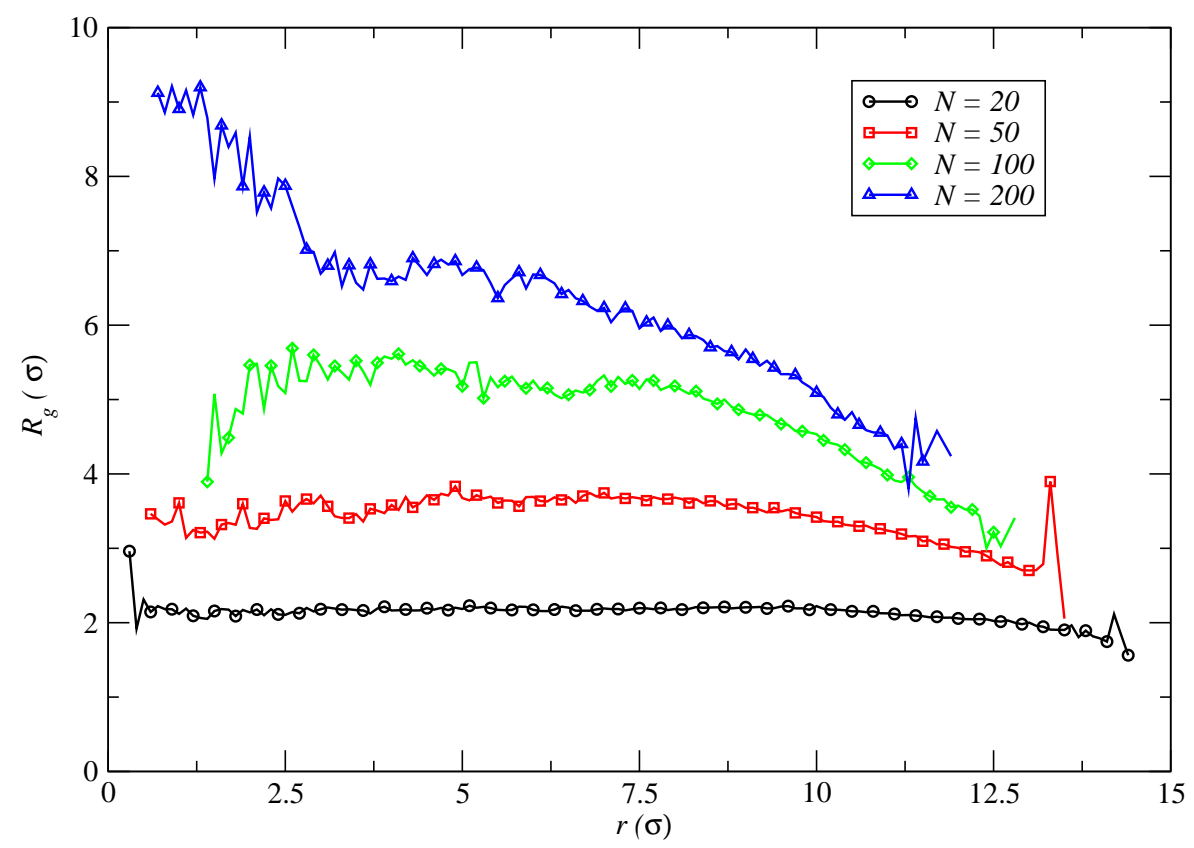

Figure A.2: The average $R_{g}$ profiles as a function of radial distance, $r$ for different chain lengths.

In Fig. A.2 we show the average chain size profile as a function of $r$, for the 8000 monomer droplet case, for various chain compositions, $N$. The profiles are smooth for shorter chains and the noise in data increases with $N$. This is purely due to the fact that there are more chains contributing to the averages, in each of the bins, in the small $N$ regime than in large $N$ regime. The noise in $R_{g}$ close to the surfaces is due to the fact there are fewer chains in these regions and hence poor statistics. In general, as we move away from the centre towards the droplet surface the average chain size decreases. We also see, the rate at which $R_{g}$ decreases as a function of $r$, increases with $N$. This is reasonable as the number of neighbouring chains decreases rapidly with increasing $N$ and hence the chains prefer to shrink. In all cases, as $r \rightarrow R_{d}$, where $R_{d}$ is the radius of the droplet, $R_{g}$ decreases compared to its value in the bulk. We attribute this to the surface tension. 


\section{Bibliography}

[1] G. G. Pereira, "Effect of variable slip boundary conditions on flows of pressure driven non-newtonian fluids," J. Non-Newtonian Fluid Mech., vol. 157, pp. 197-206, 2009.

[2] S. S. Pickles, "The constitution and synthesis of caoutchouc," J. Chem. Soc. Trans., vol. 97, pp. 1085-1090, 1910.

[3] H. Staudinger and J. Fritschi, "On isoprene and rubber - fifth report - on the hydration of rubber and on its constitution," Helvetica Chimica Acta, vol. 5, pp. 785-806, 1922.

[4] P. J. Flory, Principles of Polymer Chemistry. Ithaca, NY: Cornell University Press, 1953.

[5] M. L. Huggins, "Theory of solutions of high polymers," J. Am. Chem. Soc., vol. 64, pp. 1712-1719, 1942.

[6] P. G. de Gennes, "Soft matter," Rev. Mod. Phys., vol. 64, pp. 645-648, 1992.

[7] S. Nayak and L. A. Lyon, "Soft nanotechnology with soft nanoparticles," Angew. Chem. Int. Ed., vol. 44, pp. 7686-7708, 2005.

[8] I. W. Hamley, "Nanotechnology with soft materials," Angew. Chem. Int. Ed., vol. 42, pp. 1692-1712, 2003.

[9] F. W. Starr and S. C. Glotzer, "Molecular and mesoscale simulations of nanofilled polymers," Mater. Res. Soc. Symp. Series, 661, p. 1, 2001.

[10] J. M. Oh, T. Faez, S. de Beer, and F. Mugele, "Capillarity-driven dynamics of water-alcohol mixtures in nanofluidic channels," Microfluid Nanofluid., vol. 9, pp. 123-129, 2009.

[11] G. H. Hu, S. Hoppe, L. F. Feng, and C. Fonteix, "Nano-scale phenomena and applications in polymer processing," Chem. Eng. Sci., vol. 62, pp. 3528-3537, 2007.

[12] S. P. Adiga and D. W. Brenner, "Flow control through polymer-grafted smart nanofluidic channels: Molecular dynamics simulations," Nano Lett., vol. 5, pp. 2509-2514, 2005.

[13] G. G. Pereira, "Towards nano-scale devices via self-assembly," Curr. Appl. Phys., vol. 4, pp. 255-258, 2004.

[14] M. Kotelyanskii and D. N. Theodorou, Simulation Methods for Polymers. New York: Marcel Dekker, Inc, 2004. 
[15] K. Binder, Monte Carlo and Molecular Dynamics Simulations in Polymer Science. New York: Oxford University Press, 1995.

[16] F. Müller-Plathe, "Coarse-graining in polymer simulation: From the atomistic to the mesoscopic scale and back," Chem. Phys. Chem., vol. 3, pp. 754-769, 2002.

[17] S. C. Glotzer and W. Paul, "Molecular and mesoscale simulation methods for polymer materials," Annu. Rev. Mater. Res., vol. 32, pp. 401-436, 2002.

[18] K. Binder and A. Milchev, "Off-lattice monte carlo methods for coarse-grained models of polymeric materials and selected applications," J. Comput-Aided Mater. Des., vol. 9, pp. 33-74, 2002.

[19] S. Girard and F. Müller-Plathe, "Coarse-graining in polymer simulations," Lect. Note. Phys., vol. 640, pp. 327-356, 2004.

[20] H. Bruus, Theoretical Microfluidics. USA: Oxford University Press, 2007.

[21] T. M. Squires and S. R. Quake, "Microfluidics: Fluid physics at nanoliter scale," Rev. Mod. Phys., vol. 77, pp. 977-1026, 2005.

[22] D. J. Beebe, G. A. Mensing, and G. M. Walker, "Physics and applications of microfluidics in biology," Annu. Rev. Biomed. Eng., vol. 4, pp. 261-286, 2002.

[23] H. A. Stone, A. D. Stroock, and A. Ajdari, "Engineering flows in small devices : Microfluidics toward a lab-on-a-chip," Annu. Rev. Fluid Mech., vol. 36, pp. 381-411, 2004.

[24] J. M. Ottino and S. Wiggins, "Introduction: mixing in microfluidics," Philos. Trans. R. Soc. London. Ser. A, vol. 362, pp. 923-935, 2004.

[25] S. C. Hendy, M. Jasperse, and J. Burnell, "The effect of patterned slip on micro and nanofluidic flows," Phys. Rev. E, vol. 65, p. 016303, 2005.

[26] J. Ou, G. R. Moss, and J. P. Rothstein, "Enhanced mixing in laminar flows using ultrahydrophobic surfaces," Phys. Rev. E, vol. 65, p. 031502, 2007.

[27] T. Young, "An essay on the cohesion of fluids," Philos. Trans. R. Soc. London, vol. 95, pp. 65-87, 1805.

[28] P. S. Laplace, Méchanique céleste, vol. 10. 1806.

[29] R. Lucas, "Ueber das zeitgesetz des kapillaren aufstiegs von flussigkeiten," Kolloid Z, vol. 23, pp. 15-22, 1918.

[30] E. W. Washburn, “The dynamics of capillary flow," Phys. Rev., vol. 17, pp. 273-283, 1921.

[31] D. I. Dimitrov, A. Milchev, and K. Binder, "Capillary rise in nanopores: Molecular dynamics evidence for the lucas-washburn equation," Phys. Rev. Lett., vol. 99, p. 054501, 2007.

[32] B. V. Zhmud, F. Tiberg, and K. Hallstensson, "Dynamics of capillary rise," J. Colloid and Interface Sci., vol. 228, pp. 263-269, 2000.

[33] G. Martic, F. Genter, D. Seveno, D. Coulon, and J. D. Coninck, "A molecular dynamics simulation of capillary imbibition," Langmuir, vol. 18, pp. 7971-7976, 2002. 
[34] S. Supple and N. Quirke, "Rapid imbibition of fluids in carbon nanotubes," Phys. Rev. Lett., vol. 90, p. 214501, 2003.

[35] A. Marmur, "Penetration of a small drop into a capillary," J. Colloid and Interface Sci., vol. 122, pp. 209-219, 1988.

[36] A. Marmur, "Penetration and displacement in capillary systems of limited size," Adv. Colloid. Interf. Sci., vol. 39, pp. 13-33, 1992.

[37] D. Schebarchov and S. C. Hendy, "Capillary absorption of metal nanodroplets by single-wall carbon nanotubes," Nano Lett., vol. 8, pp. 2253-2257, 2008.

[38] R. Kimmich, N. Fatkullin, C. Mattea, and E. Fischer, "Polymer chain dynamics under nanoscopic confinements," Magnetic Resonance Imaging, vol. 23, pp. 191-196, 2005.

[39] E. Kim, Y. Xia, and G. M. Whitesides, "Polymer microstructures formed by moulding in capillaries," Nature, vol. 376, pp. 581-584, 1995.

[40] M. Zhang, P. Dobriyal, J.-T. Chen, and T. P. Russell, "Wetting transition in cylindrical alumina nanopores with polymer melts," Nano. Lett., vol. 6, pp. 1075-1079, 2006.

[41] M. Böltau, S. Walheim, J. Mlynek, G. Krausch, and U. Steiner, "Surface-induced structure formation of polymer blends on patterned substrates," Nature, vol. 391, pp. 877-879, 1998.

[42] K. Binder, "Surface effects on polymer blends and block copolymer melts: Theoretical concepts of surface enrichment, surface induced phase separation and ordering," Acta Polym, vol. 46, pp. 204-225, 1995.

[43] A. Budkowski, A. Bernasik, P. Cyganik, J. Raczkowska, B. Penc, B. Bergues, K. Kowalski, J. Rysz, and J. Janik, "Substrate-determined shape of free surface profiles in spin-cast polymer blend films," Macromolecules, vol. 36, pp. 4060-4067, 2003.

[44] K. S. Schweizer and J. G. Curro, "Prism theory of the structure, thermodynamics, and phase transitions of polymer liquids and alloys," Adv. Polym. Sci., vol. 116, pp. 319-377, 1994.

[45] S. K. Kumar, M. Vacatello, and D. Y. Yoon, "OffâĂ Ǩlattice monte carlo simulations of polymer melts confined between two plates," J. Chem. Phys., vol. 89, pp. 5206-5215, 1988.

[46] M. Rubinstein and R. H. Colby, Polymer Physics. USA: OXFORD UNIVERSITY PRESS, 2003.

[47] D. R. M. Williams, "Corrugational instabilities of thin copolymer films," Phys. Rev. Lett., vol. 75, pp. 453-456, 1995.

[48] G. Coulon, B. Collin, D. Ausserre, D. Chatenay, and T. Russell, "Islands and holes on the free surface of thin diblock copolymer films. i. characteristics of formation and growth," J. de Physique, vol. 51, pp. 2801-2811, 1990.

[49] T. Mansky and T. Russell, "Free structure confinement of diblock copolymer 
multilayers," Macromolecules, vol. 28, pp. 8092-8095, 1995.

[50] M. Doi, Introduction to Polymer Physics. USA: OXFORD UNIVERSITY PRESS, 1995.

[51] P.-G. de Gennes, Scaling Concepts in Polymer Physics. Londoon: Cornell University Press, 1979.

[52] M. Doi and S. F. Edwards, The Theory of Polymer Dynamics. New York: OXFORD SCIENCE PUBLICATIONS, 2003.

[53] A. Y. Grosberg and A. R. Khokhlov, Statistical Physics of Macromolecules. USA: AIP Press, 1994.

[54] D. I. Bower, An Introduction to Polymer Physics. UK: Cambridge University Press, 2002.

[55] O. P. Nwammuo and G. C. Maitland J. Chem. Soc. Faraday Trans., vol. 88, p. 1803, 1992.

[56] R. B. Bird, R. C. Armstrong, and O. Hassager, Dynamics of Polymer Liquids Volume 2: Kinetic Thery. New York: Wiley-Interscience Publication, second ed., 1987.

[57] F. Reif, Fundamentals of Statistical and Thermal Physics. New York, USA: McGrawHill Science, 1965.

[58] F. Ercolessi, A molecular dynamics primer. ICTP, Trieste: Spring College in Computational Physics, 1997.

[59] M. P. Allen and D. J. Tildesley, Computer Simulation of Liquids. USA: Oxford University Press, 1989.

[60] M. Griebel, S. Knapek, and G. Zumbusch, Numerical Simulation in Molecular Dynamics: Numerics, Algorithms, Pallelization, Applications. Berlin: Springer, 2007.

[61] D. Frenkel and B. Smith, Understanding Molecular Simulations. USA: Academic Press, second ed., 2002.

[62] D. C. Young, Computational Chemistry: A Practical Guide for Applying Techniques to Real-World Problems. USA: John Wiley \& Sons, Inc, 2001.

[63] A. R. Leach, Molecular Modelling: Principles and Applications. England: Prentice Hall, second ed., 2001.

[64] P. Schofield, "Computer simulation studies of the liquid state," Comput. Phys. Commun., vol. 5, pp. 17-23, 1973.

[65] J. M. Haile, Molecular Dynamics Simulation: Elementory Methods. New York: JOHN WILEY AND SONS, INC, 1992.

[66] M. Born and R. Oppenheimer, "On the quantum theory of molecules," Annalen der Physik, vol. 389, pp. 457-484, 1927.

[67] J. O. Hirschfelder, C. F. Curtis, and R. B. Bird, Molecular Theory of Gases and Liquids. New York: Wiley, 1954.

[68] G. C. Maitland, M. Rigby, E. B. Smith, and W. A. Wakeham, Intermolecular Forces. Oxford: Clarendon Press, 1981.

[69] K. Kremer and G. S. Grest, “Dynamics of entangled linear polymer melts:âĂL' a 
molecularâĂ ̌̌dynamics simulation," J. Chem. Phys., vol. 92, pp. 5057-5086, 1990.

[70] L. V. Woodcock, "Isothermal molecular dynamics calculations for liquid salts," Chem. Phys. Lett., vol. 10, pp. 257-261, 1971.

[71] P. H. Hünenberger, "Thermostat algorithms for molecular dynamics simulations," Adv. Polym. Sci., vol. 173, pp. 105-149, 2005.

[72] R. Kubo, “The fluctuation-dissipation theorem," Rep. Prog. Phys., vol. 29, 1966.

[73] W. F. van Gunsteren and H. J. C. Berendsen, "Algorithms for brownian dynamics," Molec. Phys., vol. 45, pp. 637-647, 1982.

[74] T. Soddemann, B. Dünweg, and K. Kremer, "Dissipative particle dynamics: A useful thermostat for equilibrium and nonequilibrium molecular dynamics simulations," Phys. Rev. E, vol. 68, p. 046702, 2003.

[75] S. Nosé, "A molecular dynamics method for simulations in the canonical ensemble," Mol. Phys., vol. 52, pp. 255-268, 1984.

[76] W. G. Hoover, "Canonical dynamics: Equilibrium phase-space distributions," Phys. Rev. A, vol. 31, pp. 1695-1697, 1985.

[77] S. Nosé, "Dynamical behavior of a thermostated isotropic harmonic oscillator," Phys. Rev. E, vol. 47, pp. 164-177, 1993.

[78] D. J. Evans and G. P. Morriss, Statistical Mechanics of Nonequilibrium Liquids. Australia: ANU E Press, 2007.

[79] C. W. Gear, "The numerical integration of ordinary differential equations of various orders," Report ANL 7126, 1966.

[80] A. Rahman, "Correlations in the motion of atoms in liquid argon," Phys. Rev., vol. 136, pp. A405-A411, 1964.

[81] L. Verlet, "Computer experiments on classical fluids. i. thermodynamical properties of lennard-jones molecules," Phys. Rev., vol. 159, pp. 98-103, 1966.

[82] D. Beeman, "Some multistep methods for use in molecular dynamics calculations," J. Comput. Phys., vol. 20, pp. 130-139, 1976.

[83] W. C. Swope, H. C. Andersen, P. H. Berens, and K. R. Wilson, "computer simulation method for the calculation of equilibrium constants for the formation of physical clusters of molecules: Application to small water clusters," J. Chem. Phys., vol. 76, pp. 637-649, 1982.

[84] S. Plimpton, "Fast parallel algorithms for short-range molecular dynamics," J. Comput. Phys., vol. 117, pp. 1-19, 1995.

[85] J. C. Phillips, R. Braun, W. Wang, J. Gumbart, E. Tajkjorshid, E. Villa, C. Chipot, R. D. Skeel, L. Kalé, and K. Schulten, "Scalable molecular dynamics with namd," J. Comput. Chem., vol. 26, pp. 1781-1802, 2005.

[86] K. Kadau, T. C. Germann, and P. S. Lomdahl, "Molecular dynamics comes of age: 320 billion atom simulation on bluegene/1," Int. J. Mod. Phys. C, vol. 17, pp. 1755-1761, 2006.

[87] "Large-scale atomic/molecular massively parallel simulator." http: 
// lammps.sandia.gov/.

[88] http://www.mcs.anl.gov/research/projects/mpi/.

[89] N. T. Nguyen and Z. Wu, "Micromixers âĂŤ a review," J. Micromech. Microeng., vol. 15, pp. R1-R16, 2005.

[90] J. Knight, "Honey, i shrunk the lab," Nature, vol. 418, pp. 474-475, 2002.

[91] S. Pennathur, C. D. Meinhart, and H. T. Soh, "How to exploit the features of microfluidics technology," Lab Chip, vol. 8, pp. 20-22, 2008.

[92] A. D. Stroock, S. K. W. Dertinger, A. Ajdari, I. Mezić, H. A. Stone, and G. M. Whitesides, "Chaotic mixer for microchannels," Science, vol. 295, pp. 647-651, 2002.

[93] C. J. Campbell and B. A. Grzybowski, “Microfluidic mixers: from microfabricated to self-assembling devices," Phil. Trans. R. Soc. Lond. A, vol. 362, pp. 1069-1086, 2004.

[94] E. Lauga, M. P. Brenner, and H. A. Stone, Handbook of Experimental Fluid Dynamics, Chap. 15. New York: Springer, 2005.

[95] G. K. Batchelor, An Introduction to Fluid Dynamics. New York, NY: Cambridge University Press, USA.

[96] H. Lamb, Hydrodynamics. New York: Dover, 1932.

[97] C. H. Choi, K. Johan, A. Westin, and K. S. Breuer, "Apparent slip flows in hydrophilic and hydrophobic microchannels," Phys. Fluids, vol. 15, pp. 2897-2902, 2003.

[98] B. D, J. Kim, H. S. Ko, and H. C. Park, "Direct measurement of slip flows in superhydrophobic microchannels with transverse grooves," Phys. Fluids, vol. 20, p. 113601, 2008.

[99] S. Granick and Y. Zhu, "Slippery questions about complex fluids flowing past solids," Nature Mater., vol. 2, pp. 221-227, 2003.

[100] P. A. Thompson and S. M. Troian, "A general boundary condition for liquid flow at solid surfaces," Nature, vol. 389, pp. 360-362, 1997.

[101] K. B. Migler, H. Hervet, and L. Leger Phys. Rev. Lett., vol. 70, p. 287, 1993.

[102] S. G. Hatzikiriakos and J. M. Dealy J. Rheol., vol. 35, p. 497, 1991.

[103] C. Cottin-Bionne, J.-L. Barrat, L. Bocquet, and E. Charlaix, "Low-friction flows of liquid at nanopatterned interfaces," Nature Mater., vol. 2, pp. 237-240, 2003.

[104] R. Pit, H. Herver, and L. Lèger, "Direct experimental evidence of slip in hexadecane: solid interfaces," Phys. Rev. Lett., vol. 85, pp. 980-983, 2000.

[105] Y. Zhu and S. Granick, "Limits of the hydrodynamic no-slip boundary condition," Phys. Rev. Lett., vol. 88, p. 106102, 2002.

[106] G. Nagayama and P. Cheng, "Effects of interface wettability on microscale flow by molecular dynamics simulation," Int. J. Heat Mass Transfer., vol. 47, pp. 501-513, 2004.

[107] Y. Zhu and S. Granick, "Rate-dependent slip of newtonian liquid at smooth 
surfaces," Phys. Rev. Lett., vol. 87, p. 096105, 2001.

[108] R. Khare, J. J. de Pablo, and A. Yethiraj, "Rheology of confined polymer melts," Macromolecules, vol. 29, pp. 7910-7918, 1996.

[109] G. S. Grest and K. Kremer, "Molecular dynamics simulation for polymers in the presence of a heat bath," Phys. Rev. A, vol. 33, pp. 3628-3631, 1986.

[110] P. A. Thompson and M. O. Robbins, "Shear flow near solids: Epitaxial order and flow boundary conditions," Phys. Rev. A, vol. 41, pp. 6830-6837, 1990.

[111] R. Auhl, R. Everaers, G. S. Grest, K. Kremer, and S. J. Plimpton, "Equilibration of long chain polymer melts in computer simulations," J. Chem. Phys., vol. 119, p. 12718, 2003.

[112] N. V. Priezjev and S. M. Troian, "Molecular origin and dynamic behavior of slip in sheared polymer films," Phys. Rev. Lett., vol. 92, p. 018302, 2004.

[113] A. Niavarani and N. V. Priezjev, "Slip boundary conditions for shear flow of polymer melts past atomically flat surfaces," Phys. Rev. E, vol. 77, p. 041606, 2008.

[114] J. H. Irving and J. G. Kirkwood, "The statistical mechanical theory of transport processes. iv. the equations of hydrodynamics," J. Chem. Phys., vol. 18, pp. 817-829, 1950.

[115] A. Jabbarzadeh, J. D. Atkinson, and R. I. Tanner, "Effect of molecular shape on rheological properties in molecular dynamics simulation of star, h, comb, and linear polymer melts," Macromolecules, vol. 36, pp. 5020-5031, 2003.

[116] A. Jabbarzadeh, J. D. Atkinson, and R. I. Tanner, "Wall slip in the molecular dynamics simulation of thin films of hexadecane," J. Chem. Phys., vol. 110, pp. 2612-2620, 1999.

[117] T. Aoyagi and M. Doi, "Molecular dynamics simulation of entangled polymers in shear flow," Comput. Theor. Polym. Sci., vol. 10, pp. 317-321, 2000.

[118] B. D. Todd, D. J. Evans, and P. J. Daivis, "Pressure tensor for inhomogeneous fluids," Phys. Rev. E, vol. 52, pp. 1627-1638, 1995.

[119] R. A. L. Jones, Soft Condensed Matter. USA: Oxford University Press, 2002.

[120] R. E. Gaidos and R. Darby, "Numerical simulation and change in type in the developing flow of a nonlinear viscoelastic fluid," J. Non-Newtonian Fluid Mech., vol. 29, pp. 59-79, 1988.

[121] P. M. Ajayan and S. Iijima, "Capillarity-induced filling of carbon nanotubes," Nature, vol. 361, pp. 333-334, 1993.

[122] D. Kumar and R. C. Sharma, "Advances in conductive polymers," Eur. Polym. J., vol. 34, pp. 1053-1060, 1998.

[123] "Nanotech: Special issue," Sci. Am., September 2001.

[124] "Nanostructure: Special issue," Chem. Rev., vol. 7, 1999.

[125] Y. N. Xia, J. A. Rogers, K. E. Paul, and G. M. Whitesides, "Unconventional methods for fabricating and patterning nanostructures," Chem. Rev., vol. 99, pp. 1823-1848, 1999. 
[126] L. Liang, J. Liu, J. C. F. Windisch, G. J. Exarhos, and Y. H. Lin, "Direct assembly of large arrays of oriented conducting polymer nanowires," Angew. Chem., vol. 114, pp. 3817-3820, 2002.

[127] Y. Xia, P. Yang, Y. Sun, Y. Wu, B. Mayers, B. Gates, Y. Yin, F. Kim, and H. Yan, "One-dimensional nanostructures: Synthesis, characterization, and applications," Adv. Mater., vol. 15, pp. 353-389, 2003.

[128] A. N. Aleshin, "Polymer nanofibers and nanotubes: Charge transport and device applications," Adv. Mater., vol. 18, pp. 17-27, 2006.

[129] F. Zhang, T. Nyberg, and O. Inganäs, "Conducting polymer nanowires and nanodots made with soft lithography," Nano Lett., vol. 2, pp. 1373-1377, 2002.

[130] H. H. Jeong, J. H. Lee, C. S. Lee, H. Jang, Y. H. Yang, Y. H. Kim, and K. M. Huh, "Fabrication of selective anti-biofouling surface for micro/nanopatterning of proteins," Macromol. Res., vol. 18, pp. 868-875, 2010.

[131] D. Pisignano, G. Maruccio, E. Mele, L. Persano, F. D. Benedetto, and R. Cingolani, "Polymer nanofibers by soft lithography," Appl. Phys. Lett., vol. 87, p. 123109, 2005.

[132] D. He, Y. Wu, and B. Q. Xu, "Formation of 2,3-diaminophenazines and their self-assembly into nanobelts in aqueous medium," Eur. Polym. J., vol. 43, pp. 3703-3709, 2007.

[133] S. Supple and N. Quirke, "Molecular dynamics of transient oil flows in nanopores i: Imbibition speeds for single wall carbon nanotubes," J. Chem. Phys., vol. 121, p. 8571, 2004.

[134] S. Supple and N. Quirke, "Molecular dynamics of transient oil flows in nanopores. ii. density profiles and molecular structure for decane in carbon nanotubes," J. Chem. Phys., vol. 122, p. 104706, 2005.

[135] L.-J. Yang, T.-J. Yao, and Y.-C. Tai, "The marching velocity of the capillary meniscus in a microchannel," J. Micromech. Microeng., vol. 14, pp. 220-225, 2004.

[136] P. Huber, S. Grüner, C. Schäfer, K. Knorr, and A. V. Kityk, "Rheology of liquids in nanopores: A study on the capillary rise of water, $\mathrm{n}$-hexadecane and $\mathrm{n}$ -tetracosane in mesoporous silica," Eur. J. Phys. ST., vol. 141, pp. 101-105, 2007.

[137] D. G. Legrand and G. L. G. Jr, "The molecular weight dependence of polymer surface tension," J. Colloid and Interface Sci., vol. 31, pp. 162-167, 1969.

[138] G. T. Dee and B. B. Sauer, "The molecular weight and temperature dependence of polymer surface tension: Comparison of experiment with interface gradient theory," J. Colloid and Interface Sci., vol. 152, pp. 85-103, 1992.

[139] P. G. de Gennes, “Wetting: statics and dynamics," Rev. Mod. Phys., vol. 57, pp. 827-862, 1985.

[140] J. Yaneva, A. Milchev, and K. Binder, "Dynamics of a spreading nanodroplet : A molecular dynamic simulation," Macromol. Theory Simul., vol. 12, pp. 573-581, 2003.

[141] D. R. Heine, G. S. Grest, and E. B. W. III, "Spreading dynamics of polymer 
nanodroplets," Phys. Rev. E, vol. 68, p. 061603, 2003.

[142] D. R. Heine, G. S. Grest, and E. B. W. III, "Spreading dynamics of polymer nanodroplets in cylindrical geometries," Phys. Rev. E, vol. 70, p. 011606, 2004.

[143] K. Kremer and G. Grest, "Molecular dynamics (md) simulations for polymers," J. Phys.: Condens. Matter, vol. 2, pp. SA295-SA298, 1990.

[144] D. Schebarchov and S. C. Hendy, "Dynamics of capillary absorption of droplets by carbon nanotubes," Phys. Rev. E, vol. 78, p. 046309, 2008.

[145] J. J. Monaghan, "An introduction to sph," Comput. Phys. Commun., vol. 88, pp. 89-96, 1988.

[146] L. A. Utracki, "History of commercial polymer alloys and blends," Polym. Eng. Sci., vol. 35, pp. 2-17, 1995.

[147] J. W. Barlow and D. R. Paul, "Polymer blends and alloys - a review of selected considerations," Polym. Eng. Sci., vol. 21, pp. 985-996, 1981.

[148] D. Schwahn, "Critical to mean field crossover in polymer blends," Adv. Polym. Sci., vol. 183, pp. 1-61, 2005.

[149] K. Binder, "Phase transitions in polymer blends and block copolymer melts: Some recent developments," Adv. Polym. Sci., vol. 112, pp. 181-299, 1994.

[150] M. A. Kotnis and M. Muthukumar, "Entropy-induced frozen morphology in unstable polymer blends," Macromolecules, vol. 25, pp. 1716-1724, 1992.

[151] M. Wendlandt, T. Kerle, M. Heuberger, and J. Klein, "Phase separation in thin films of polymer blends: The influence of symmetric boundary conditions," J. Polym. Sci., Part B: Polym. Phys., vol. 38, pp. 831-837, 2000.

[152] H. Morita, T. Kawakatsu, and M. Doi, "Dynamic density functional study on the structure of thin polymer blend films with a free surface," Macromolecules, vol. 34, pp. 8777-8783, 2001.

[153] C. Harrison, D. H. Adamson, Z. Cheng, J. M. Sebastian, S. Sethuraman, D. A. Huse, R. A. Register, and P. M. Chaikin, "Mechanisms of ordering in striped patterns," Science, vol. 290, pp. 1558-1560, 2000.

[154] S. O. Kim, H. H. Solak, M. P. Stoykovich, N. J. Ferrier, J. J. de Pablo, and P. F. Nealey, "Epitaxial self-assembly of block copolymers on lithographically defined nanopatterned substrates," Nature, vol. 424, pp. 411-414, 2003.

[155] C. T. Black, "Self-aligned self assembly of multi-nanowire silicon field effect transistors," Appl. Phys. Lett., vol. 87, p. 163116, 2005.

[156] B. Collin, D. Chatenay, G. Coulon, D. Ausserre, and Y. Gallot, "Ordering of copolymer thin films as revealed by atomic force microscopy," Macromolecules, vol. 25, pp. 1621-1622, 1992.

[157] G. G. Pereira, D. R. M. Williams, and A. Chakrabarti, "Interfacial profiles of mismatched lamellae in thin diblock copolymer films," J. Chem. Phys., vol. 112, p. 10011, 2000.

[158] L. A. Utracki, Polymer Blends Handbook, vol. 1. Dordrecht: Kluwer Academic 
Publishers, 2002.

[159] J. W. Cahn and J. E. Hilliard, "Free energy of a nonuniform system. i. interfacial free energy," J. Chem. Phys., vol. 28, p. 258, 1958.

[160] J. W. Cahn, “On spinodal decomposition," Acta Metall., vol. 9, pp. 795-801, 1961.

[161] J. J. Monaghan Rep. Prog. Phys., vol. 68, p. 1703, 2005.

[162] G. R. Liu and M. B. Liu, Smoothed Particle Hydrodynamics: A meshfree particle method. Singapore: World Scientific Publishing, 2003.

[163] J. J. Monaghan, "Smoothed particle hydrodynamics," Annu. Rev. Astron. Astrophys., vol. 30, pp. 543-574, 1992.

[164] T. Okuzono, "Smoothed-particle method for phase separation in polymer mixtures," Phys. Rev. E, vol. 56, pp. 4416-4426, 1997.

[165] M. Doi and T. Ohta, "Dynamic coupling between stress and composition in polymer solutions and blends," J. Phys. II, vol. 2, pp. 1631-1656, 1992.

[166] P. W. Cleary, "Modelling confined multi-material heat and mass flows using sph," Applied Mathematical Modelling, vol. 22, pp. 981-993, 1998.

[167] J. J. Monaghan, "Simulating free surface flows with sph," J. Compl. Phys., vol. 110, pp. 399-406, 1994.

[168] K. Binder and K. Fried, "Asymmetric block copolymer melts near the microphase separation transition: a monte carlo simulation," Macromolecules, vol. 26, pp. 6878-6883, 1993. 